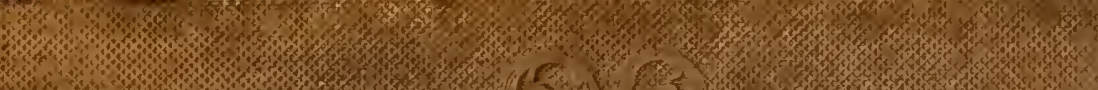

W.

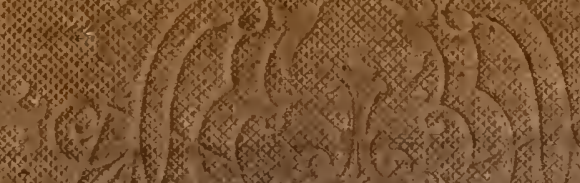

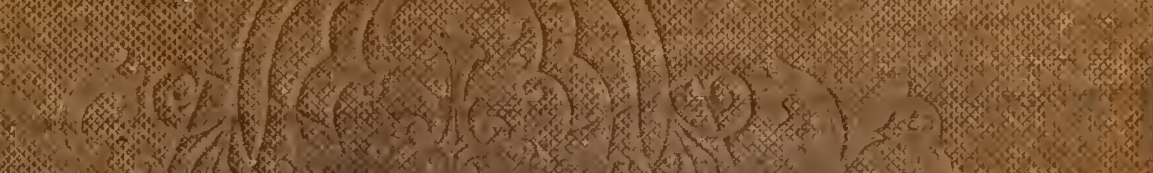

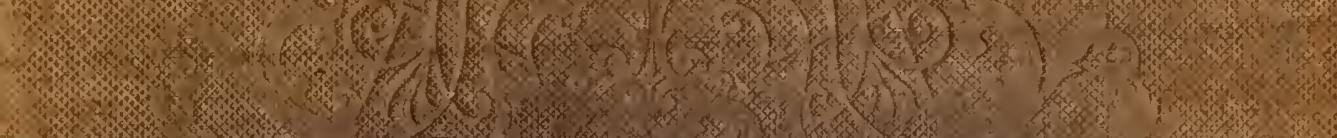

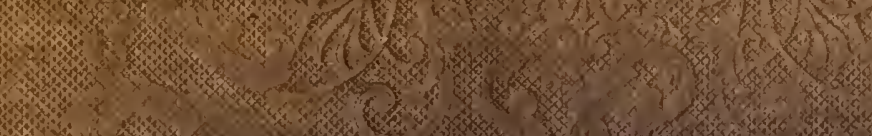

\title{
$6 \times$
}

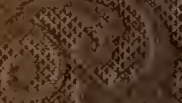
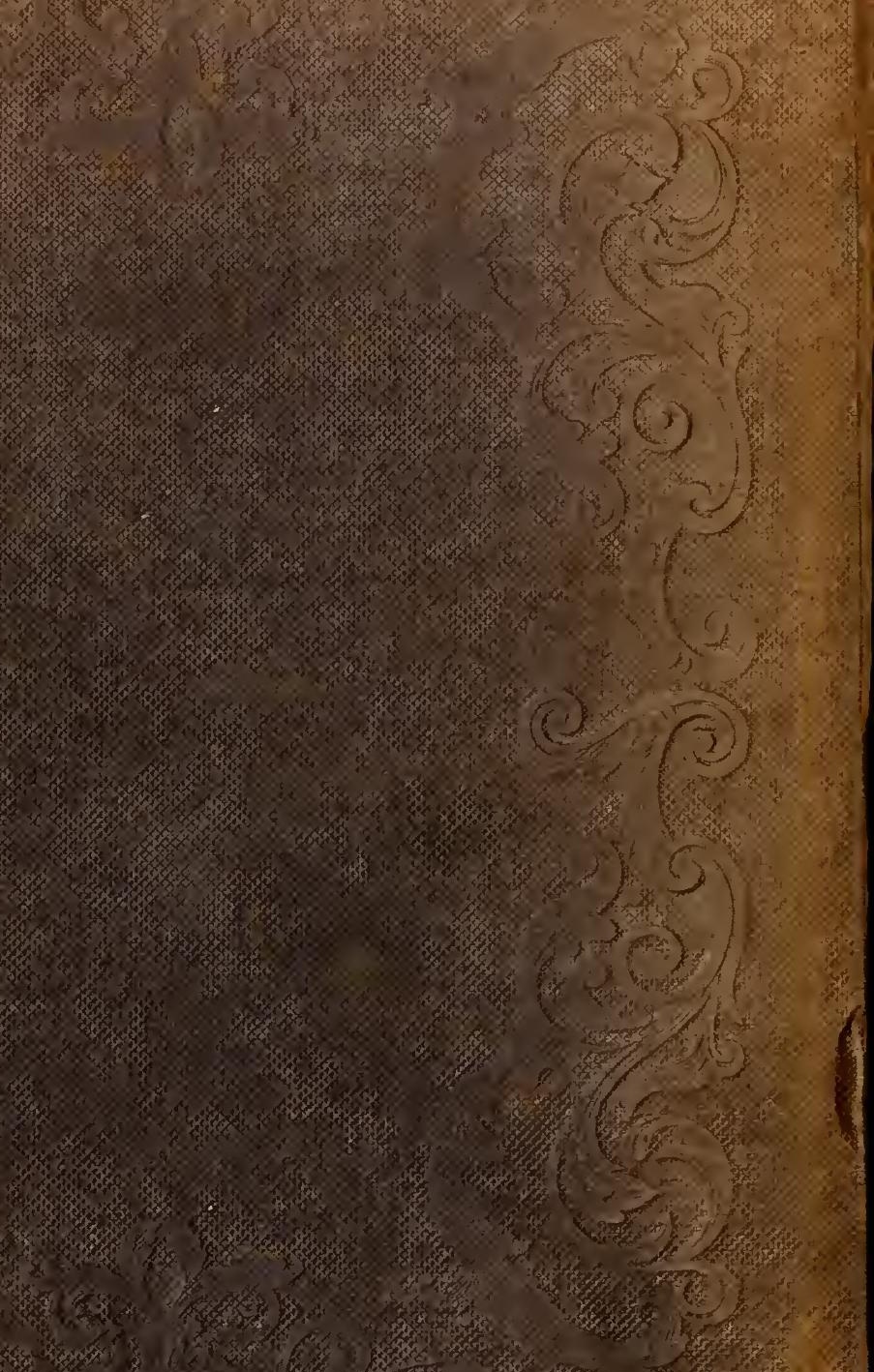

1.

$\frac{17}{4}$

$$
\text { m. }
$$

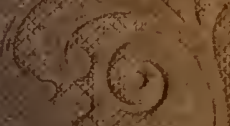$$
\text { (4) } 2
$$$$
(1) \sqrt{(2})
$$$$
3 \times 5
$$ 


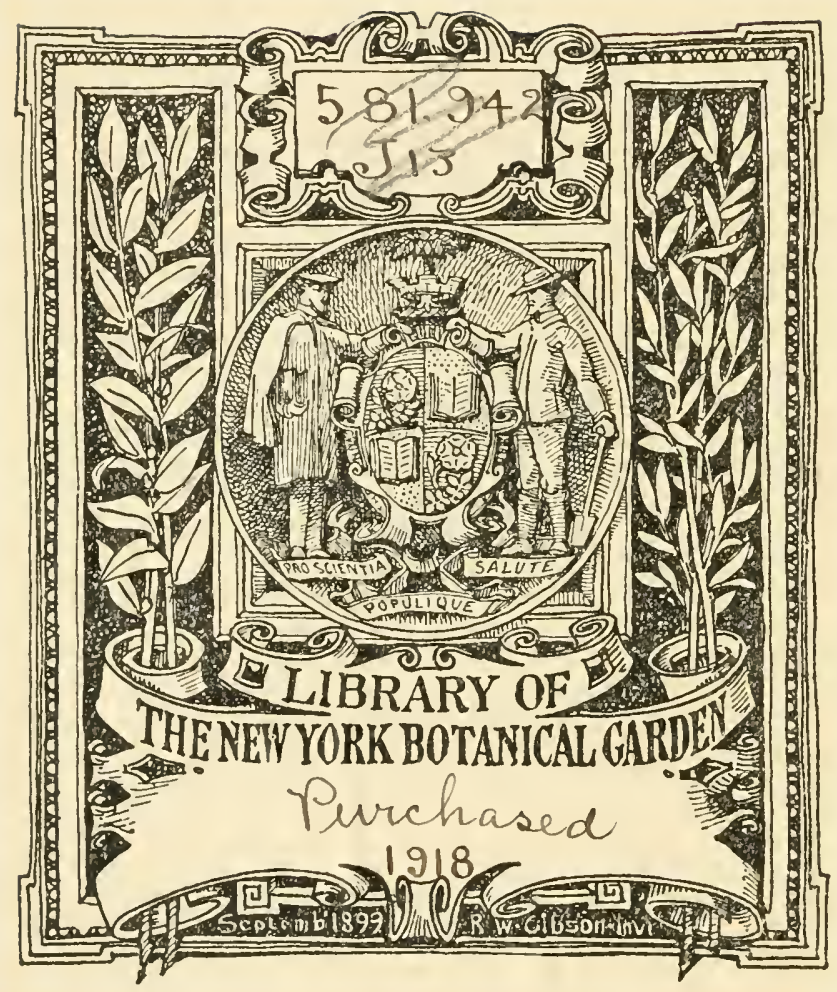


Shrom inve thathas 



$$
\begin{gathered}
+0 \times 306 \\
.33
\end{gathered}
$$




\section{PREFACE.}

THE first great discouragement of the Botanical inquirer is usually the want of some test sufficiently accurate to determine the correctness of his conclusions, when examining plants unassisted by a scientific instructor. Books of plates are, it will be readily conceded, the best and most pleasing media, by which it is possible to convey this much-needed assistance. The generality of these, however, appear not only too unwieldy for pocket-companions, and so expensive as to preclude their being put into the hands of very young persons, but also (as Sir J. E. Smith observes), from their extreme minuteness of delineation, they too often entirely supersede the employment of verbal description; and thus give rise to a species of knowledge, at once vague and unsatisfactory. But, in the present instance, it is hoped that the miniature scale which has been adopted will not only secure the great recommendations of cheapness and portability, but will also obviate the above-mentioned objection,- - the expression of character in the plants figured, furnishing the requisite degree of assistance; while their diminished size will prevent that observation of intricate minutiæ, which must inevitably give to every auxiliary the fatal facility of a royal road. This little work is, indeed, strictly intended as a manual of illustrations, and a supplement to the descriptive works of Smith, Hooker, Lindley, and Withering. 'The plates have, therefore, been left sufficiently broad in the margin to admit of their being bound with the largest of these works; while the bookbinder's knife will easily reduce them to the dimensions of the smallest.

The Appendix contains the florets of the grasses, and the fruit of the Callitriche and Umbelliferous plants, delineated on a larger scale. The figures are numbered, to correspond with the species to which they belong, as drawn in 
the body of the work. All the figures, and one Latin index, are arranged according to the Linnæan system.

Should this humble attempt be found deserving of patronage, it is in contemplation to carry forward the pioneering task thus begun, by continuing the series through Cryptogamia, so as shortly to bring before the public a cheap and complete Pictorial Encyclopædia of British Botany.

It only remains to be added, that the whole of the figures were drawn on stone by the authoress; and that the collection of specimens, from which a very large proportion of the drawings were made, was formed during the excursions of the five last summers among the vales and meadows of the midland districts; - the woods, mountains, and glens, - the rocks and sea-shores, of the northern counties, and of Wales.

LichFieid, March 1, 1839.

ERRATA IN THE FIGURES.

Dele fig. 159, 181.

For Monecia read Monoecia.

IN THE INDEX.

Read Vinca major, 382.

Vinca minor, 381.

Atriplex littoralis, 1491.

Atriplex pedunculata, 1490 .

Page 3i, eight lines from bottom, for Trifoliun resupinatum read Melilotus leucantha. 
$M C N A N I R I A$

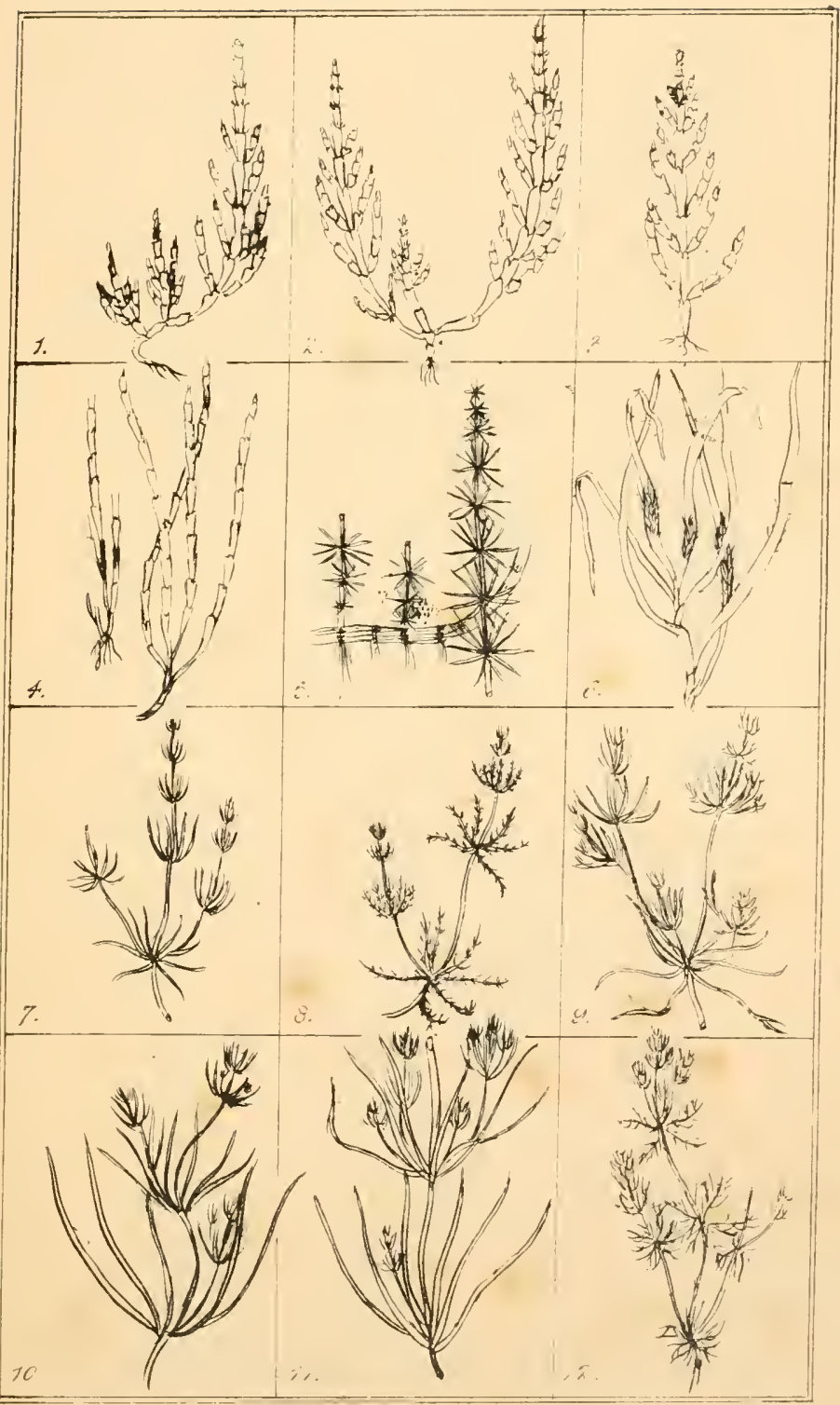



DIANDRIA

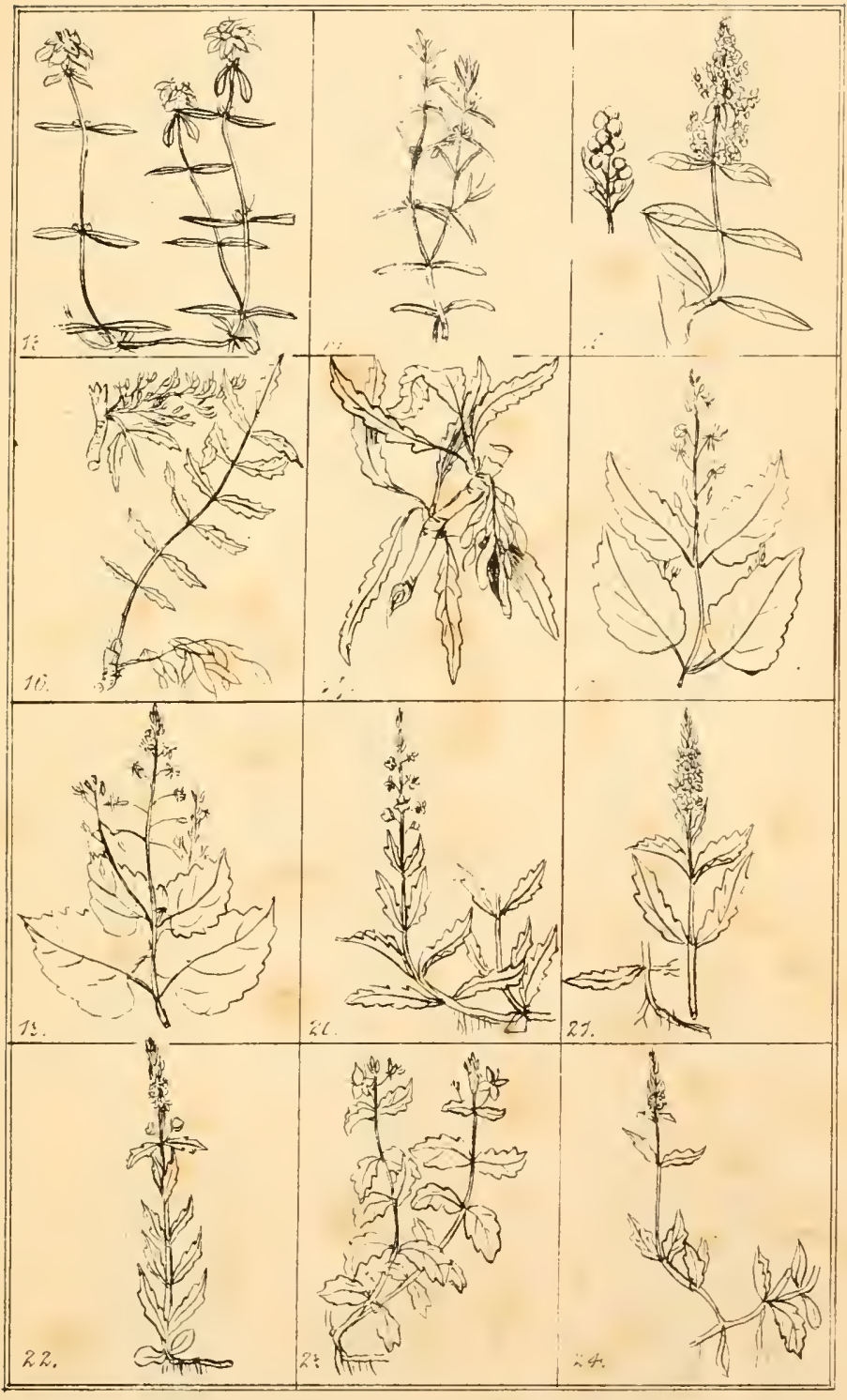





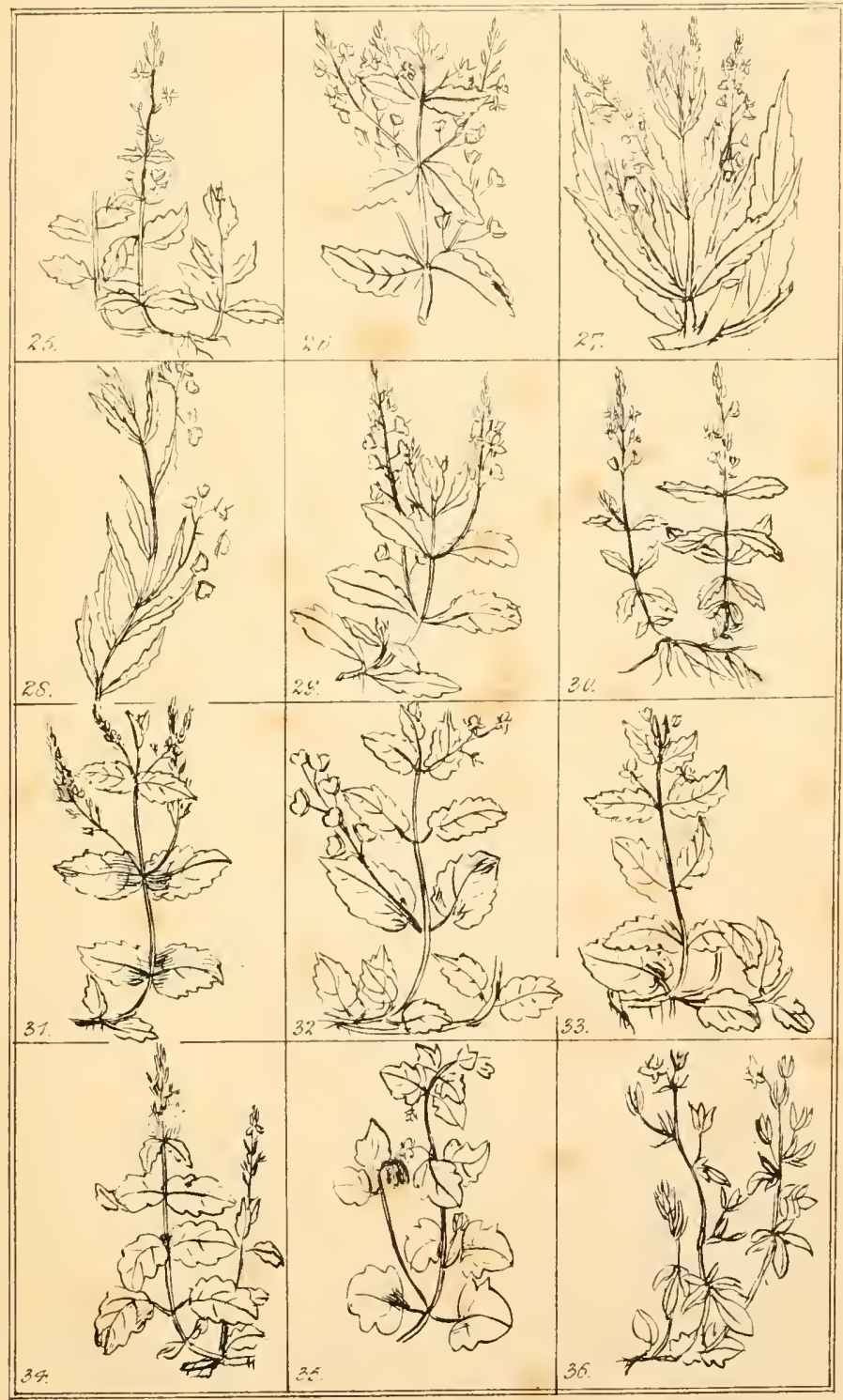





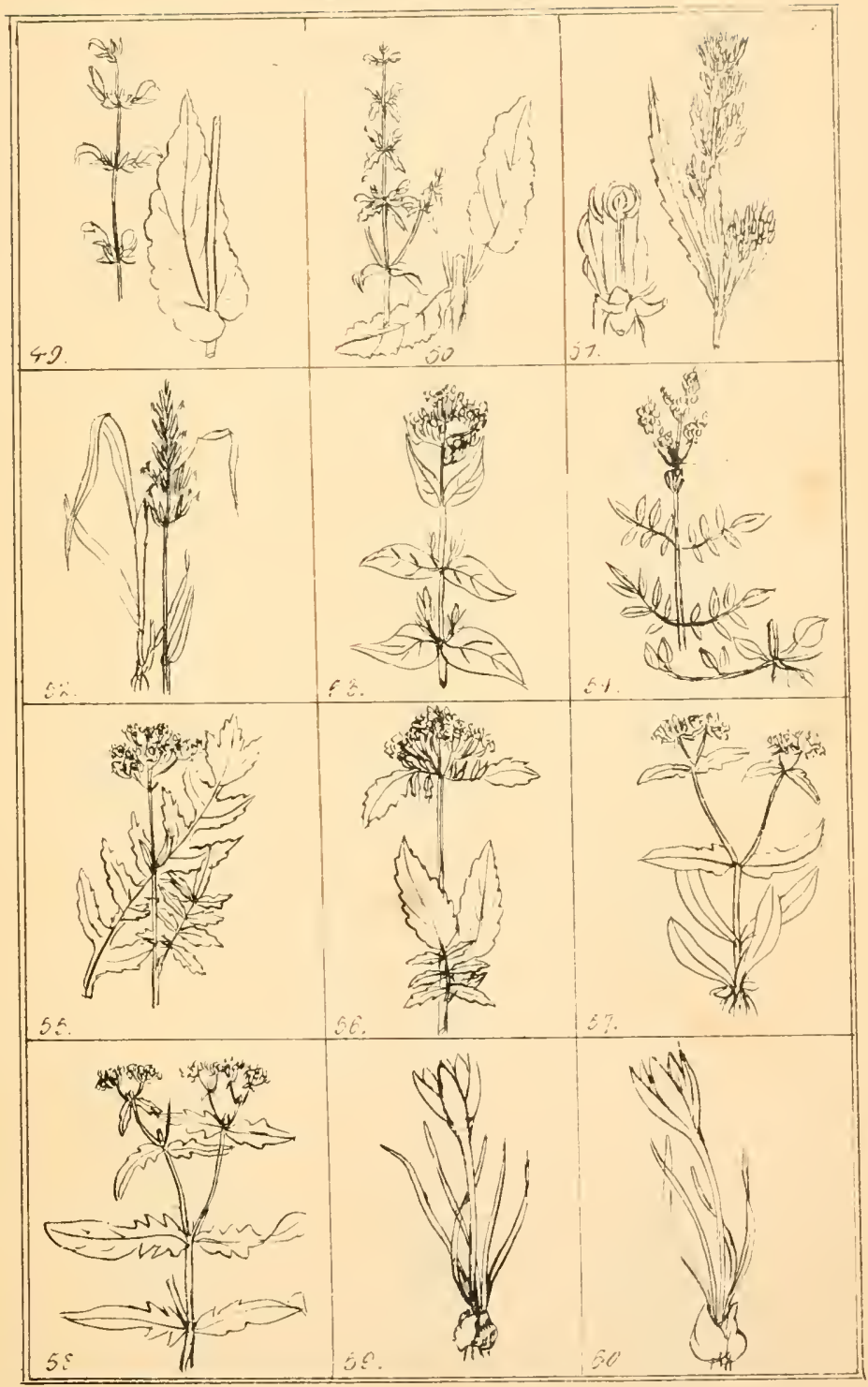





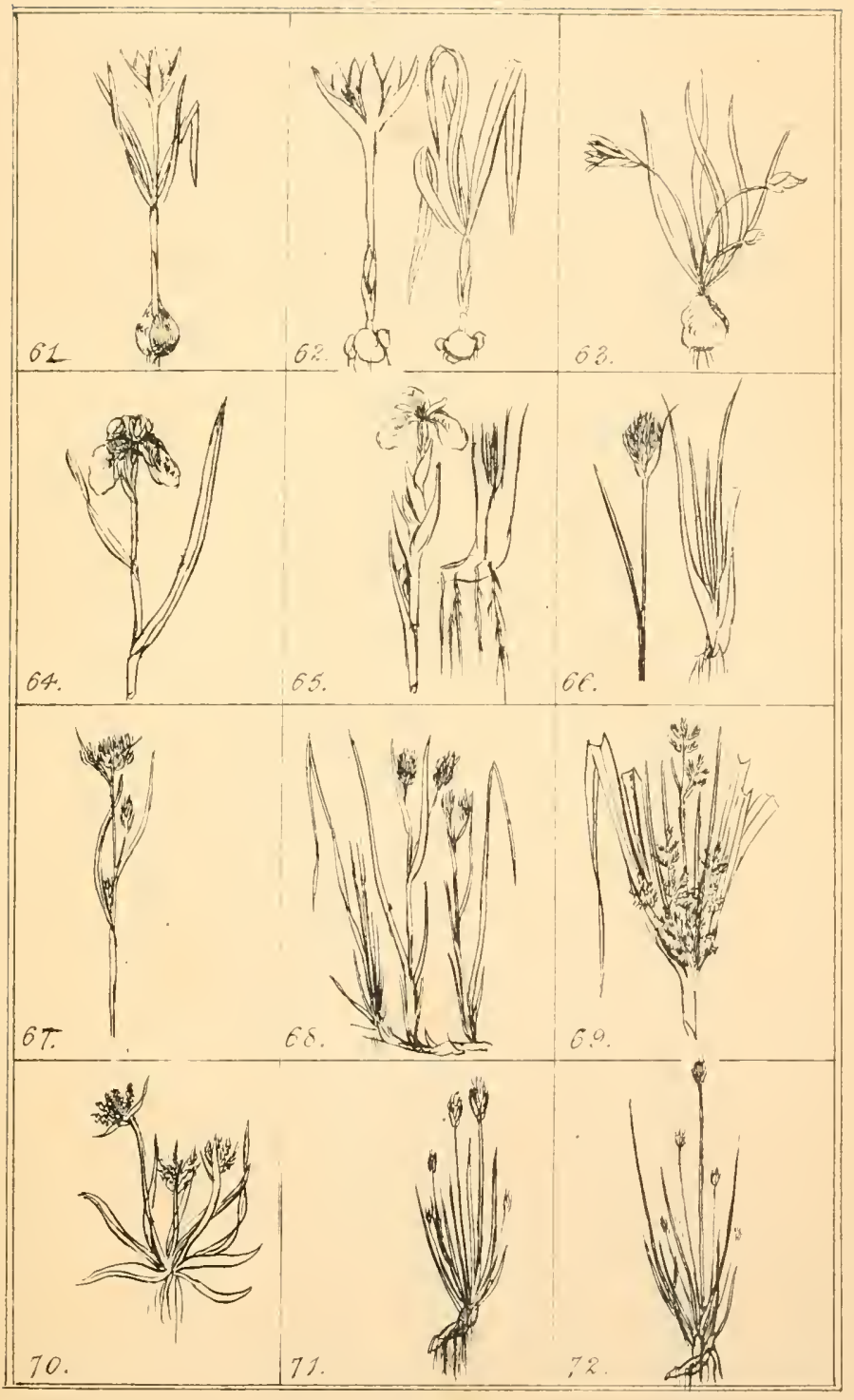





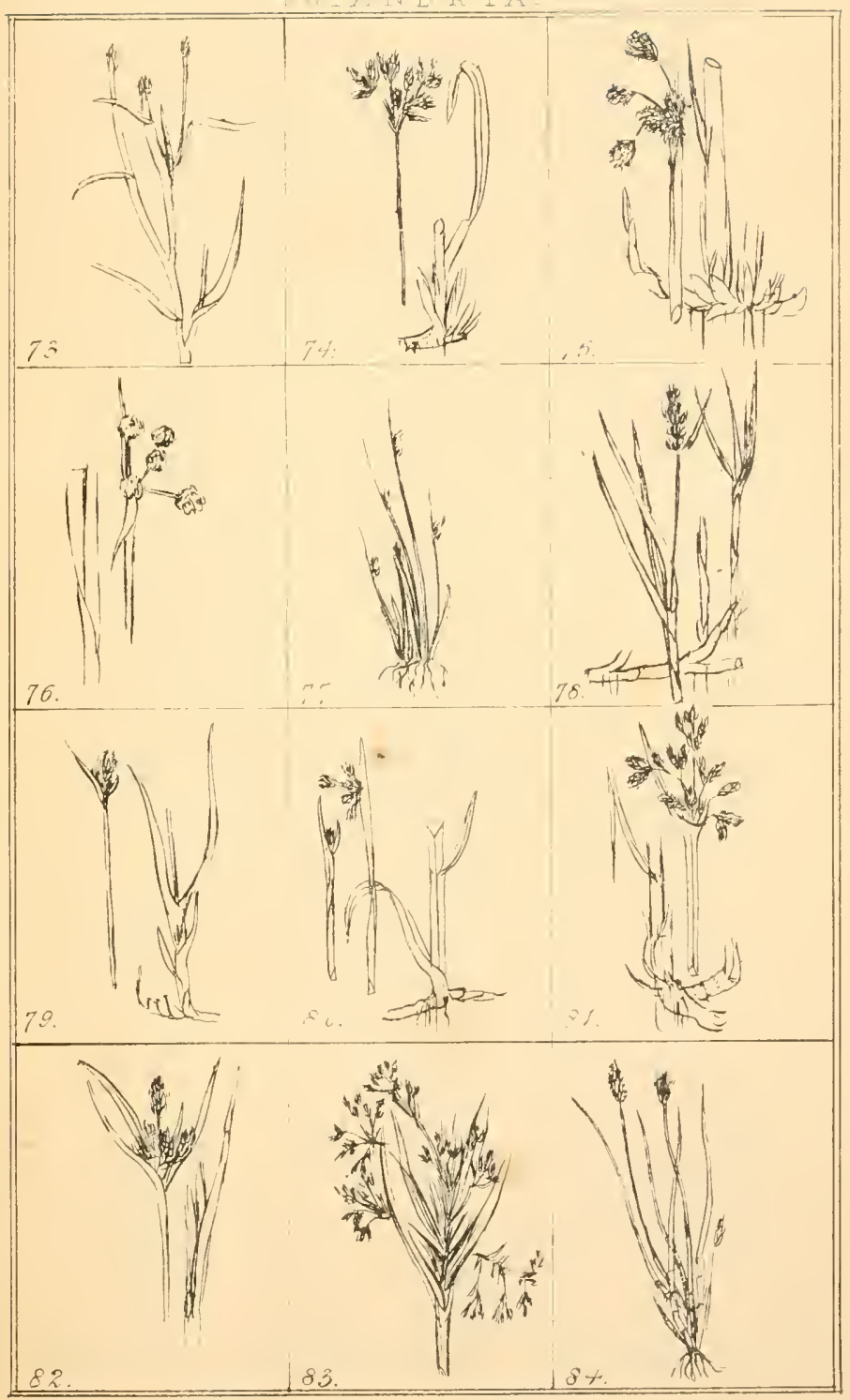



TRIAII. I I A

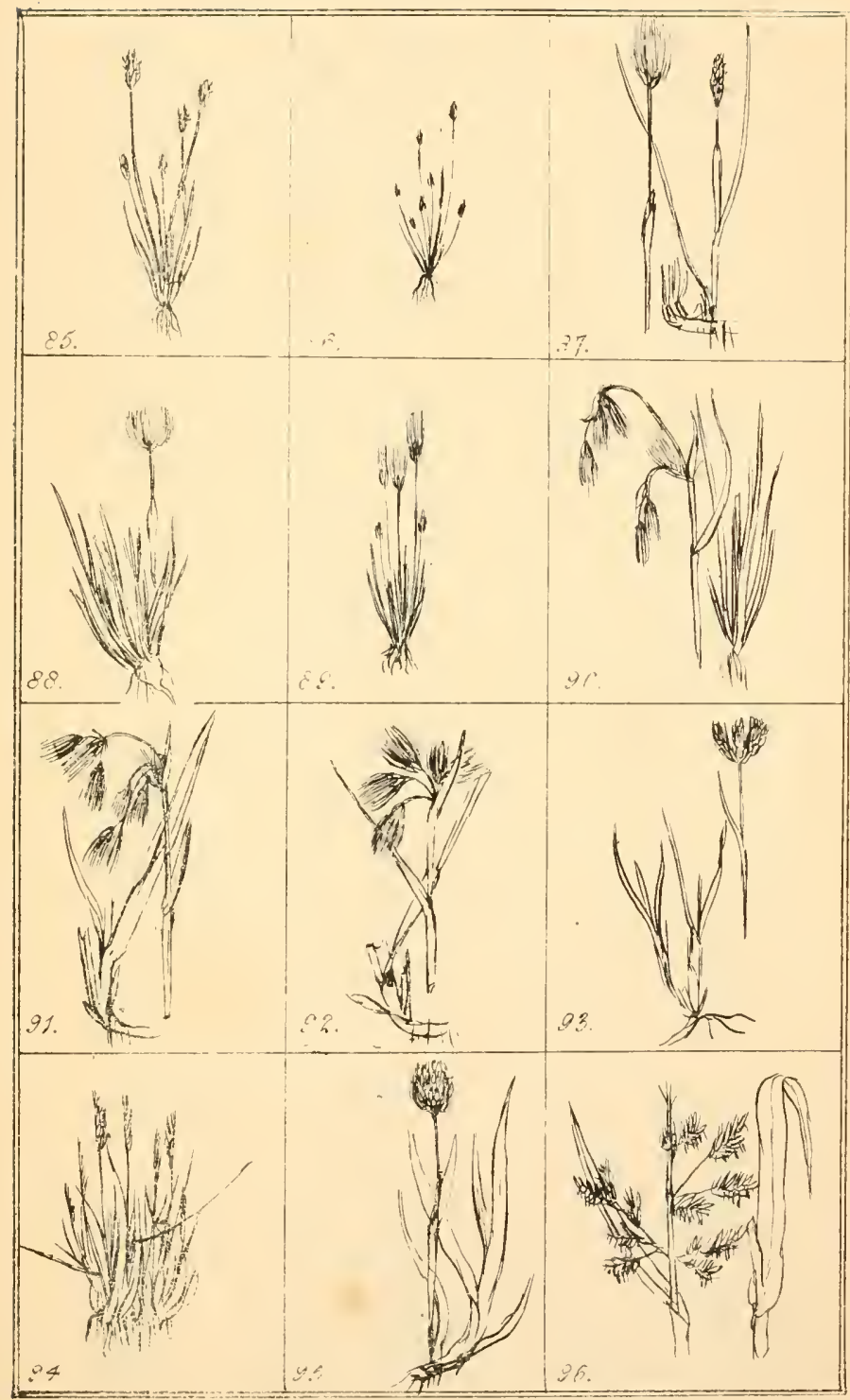



TR I A N D I A

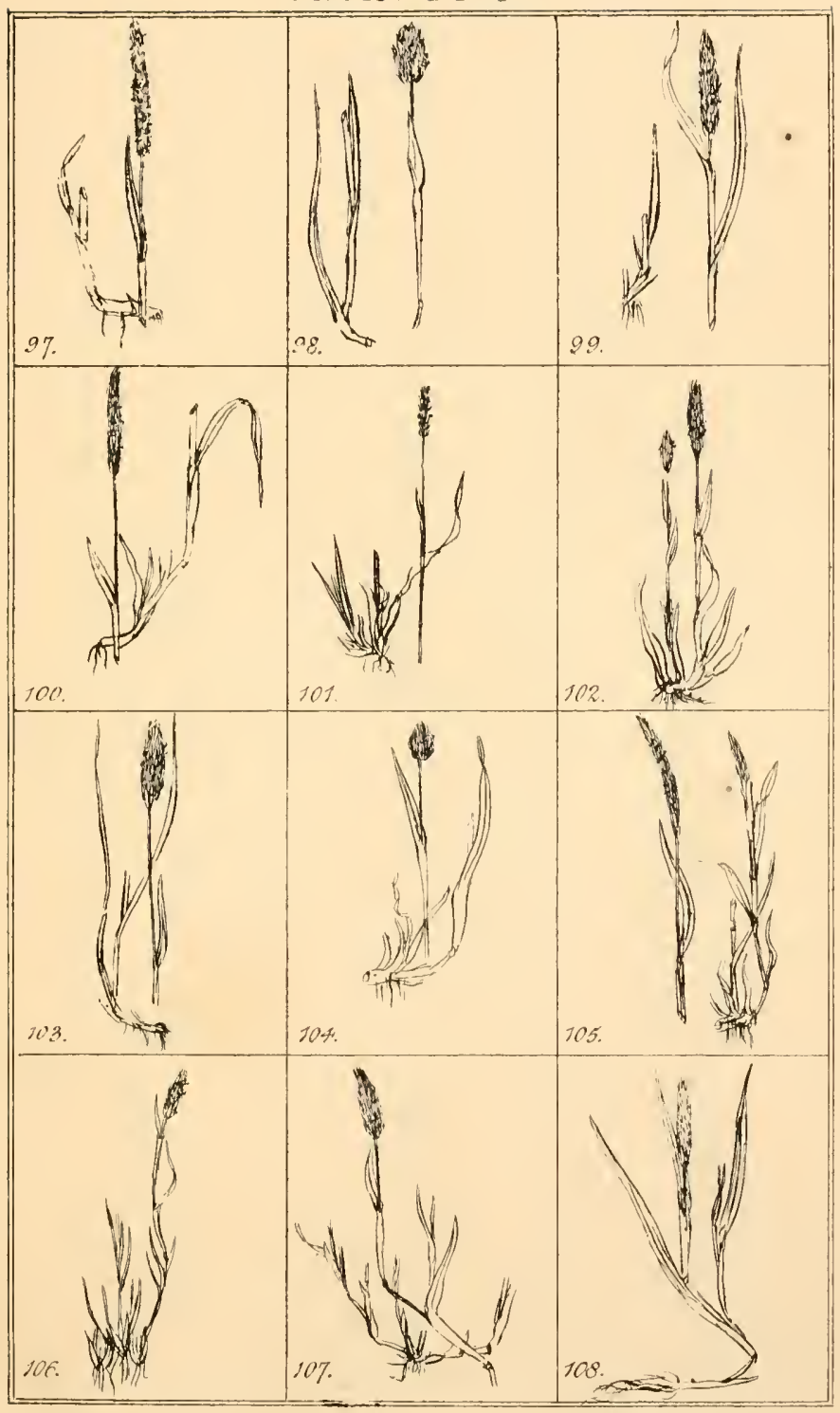





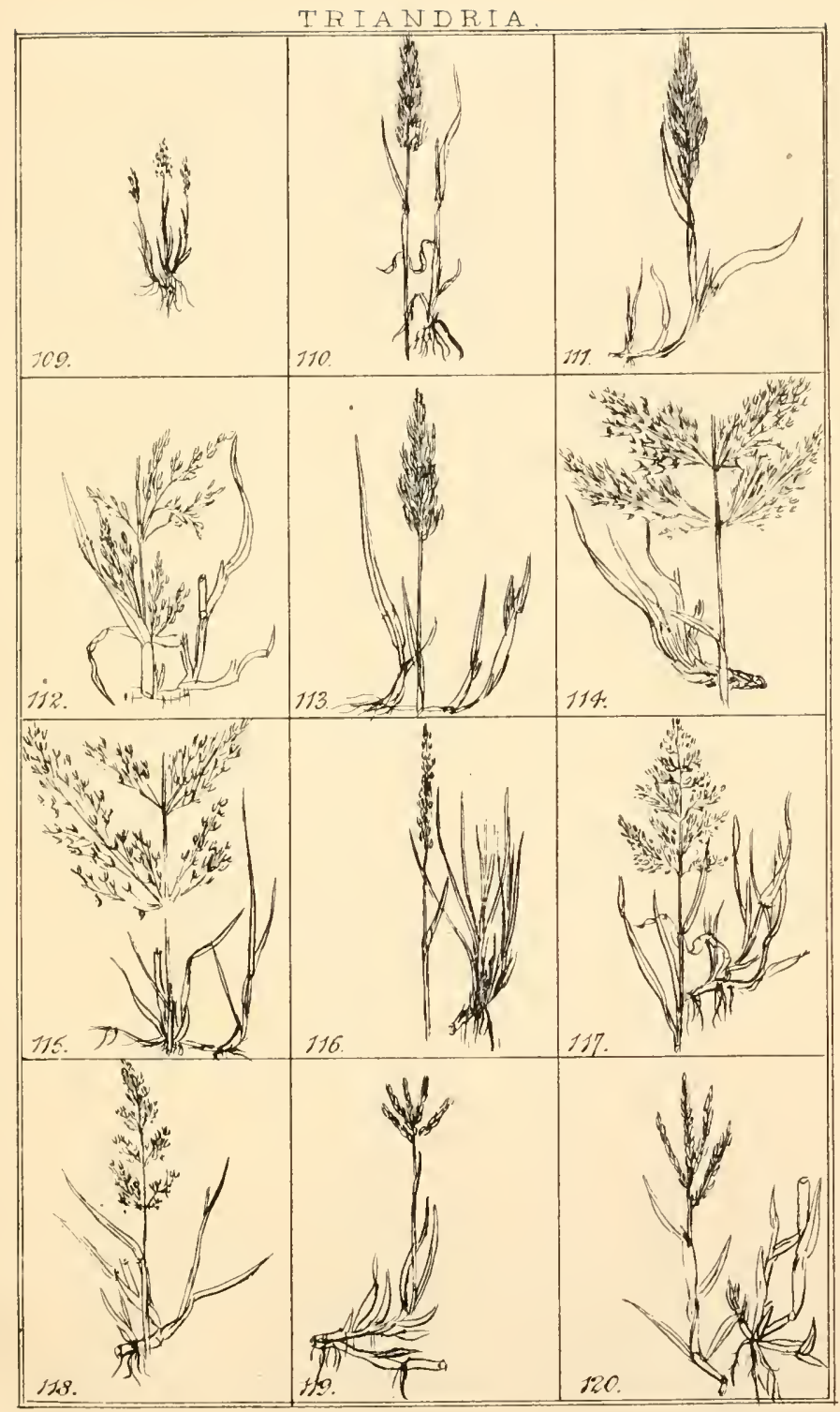



I R I A N D R I A

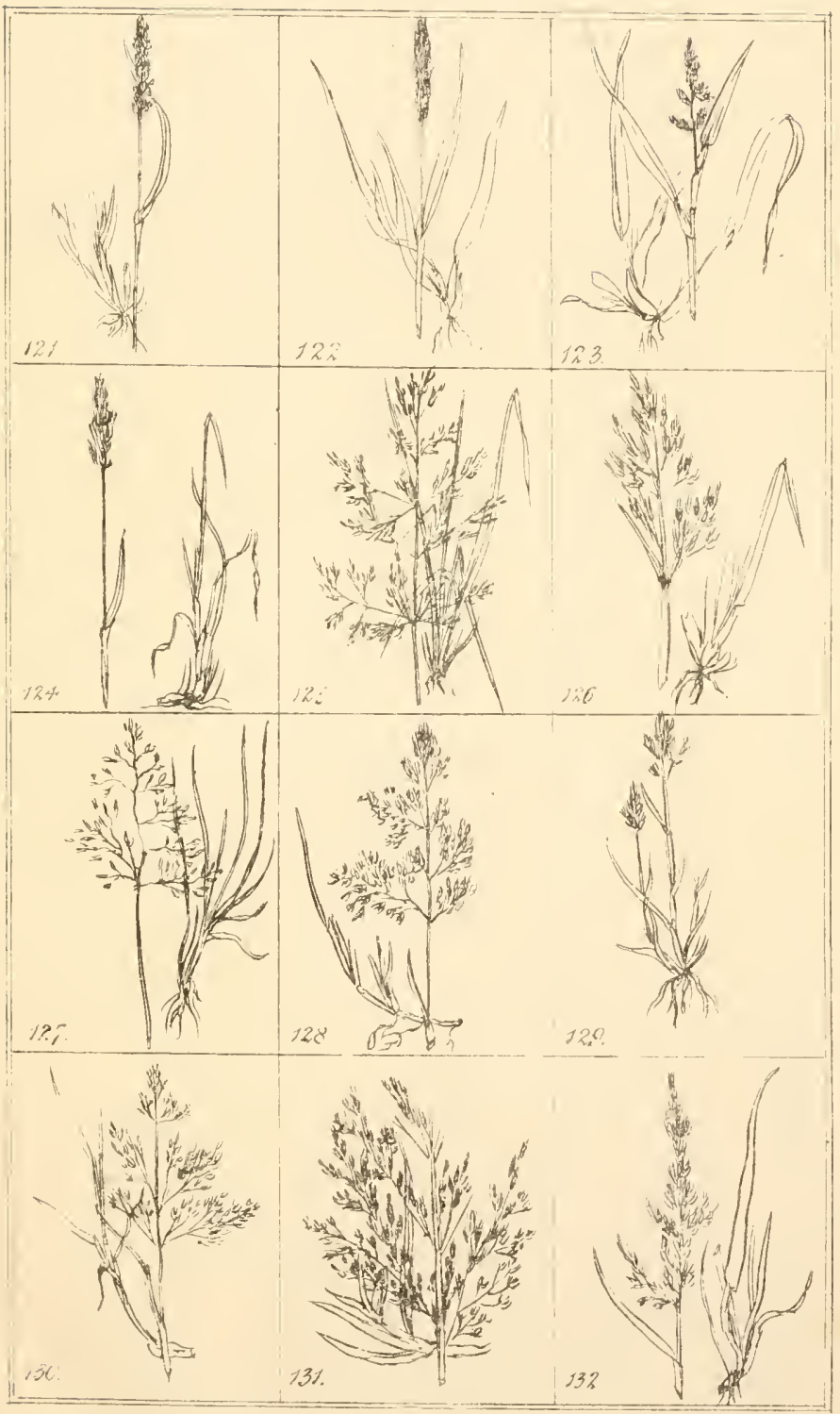





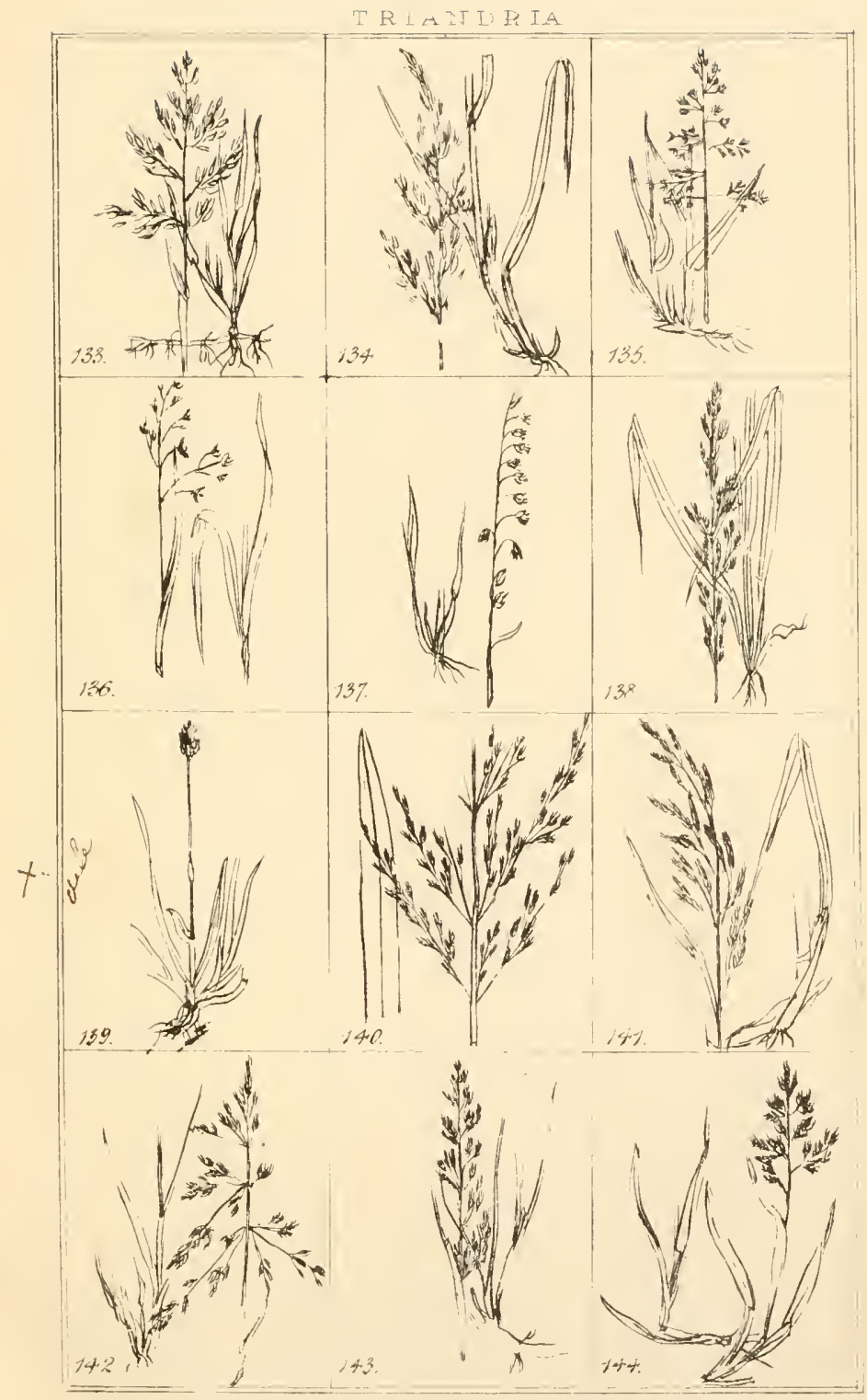





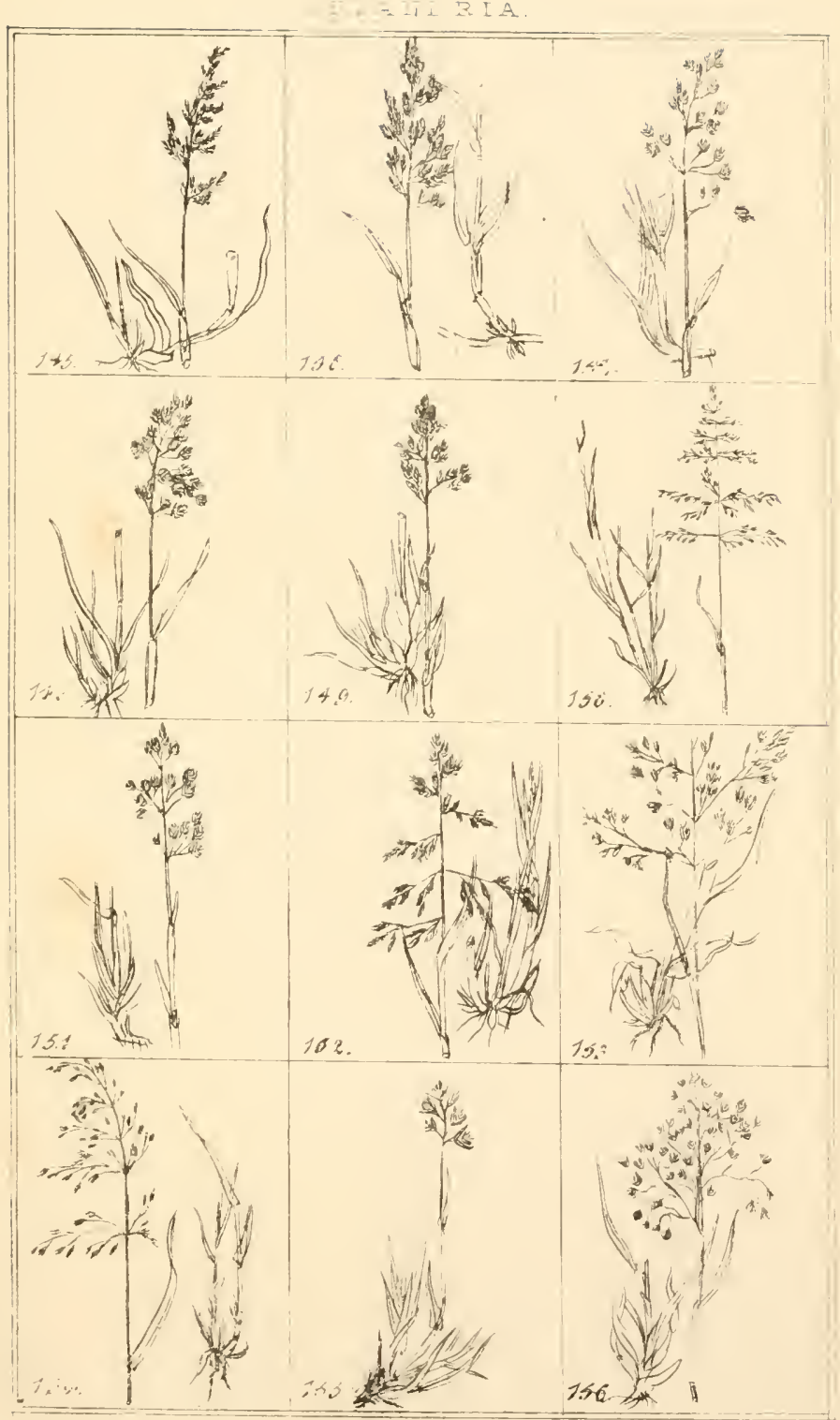





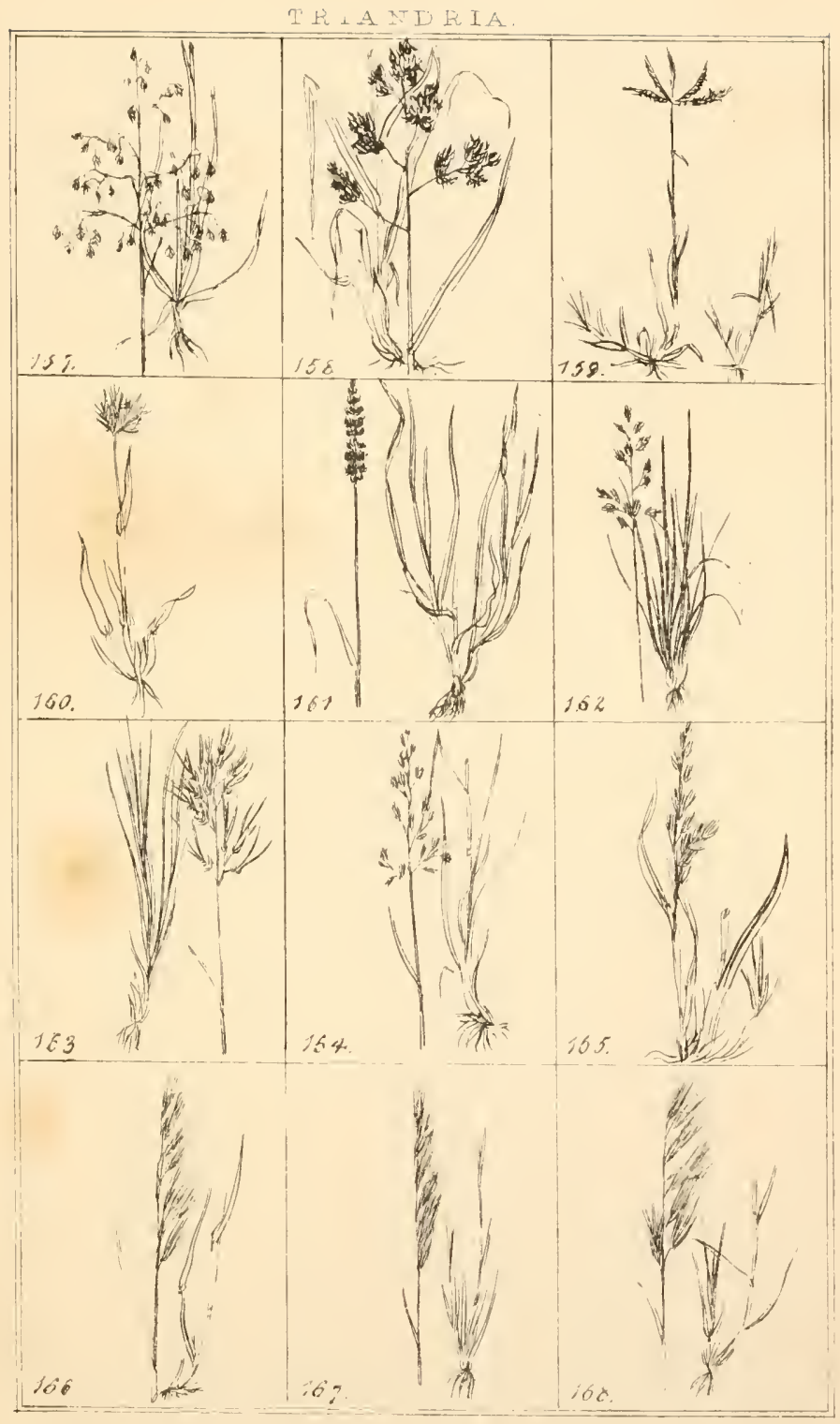



T RIANDR IA

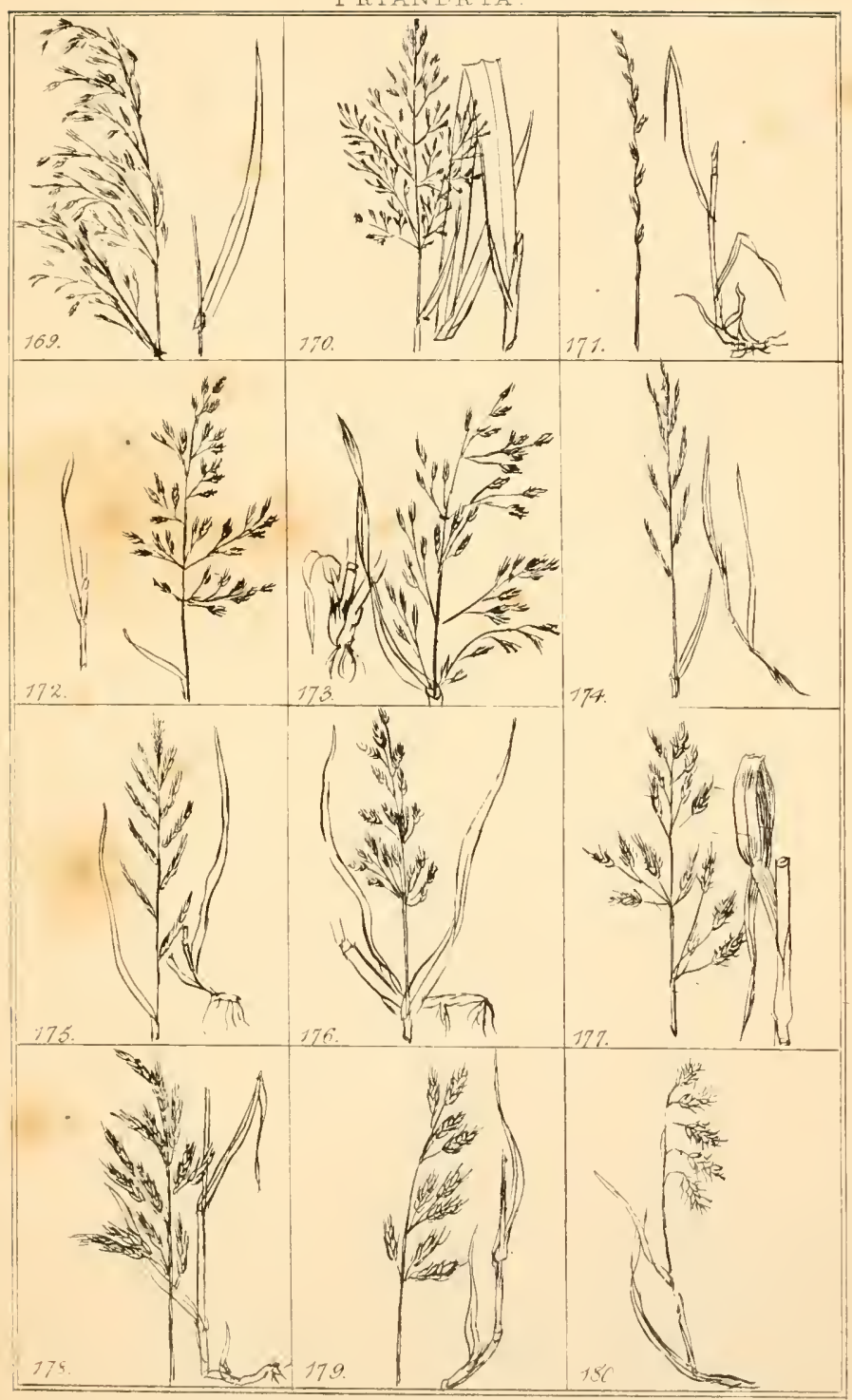



IRIANDRIA

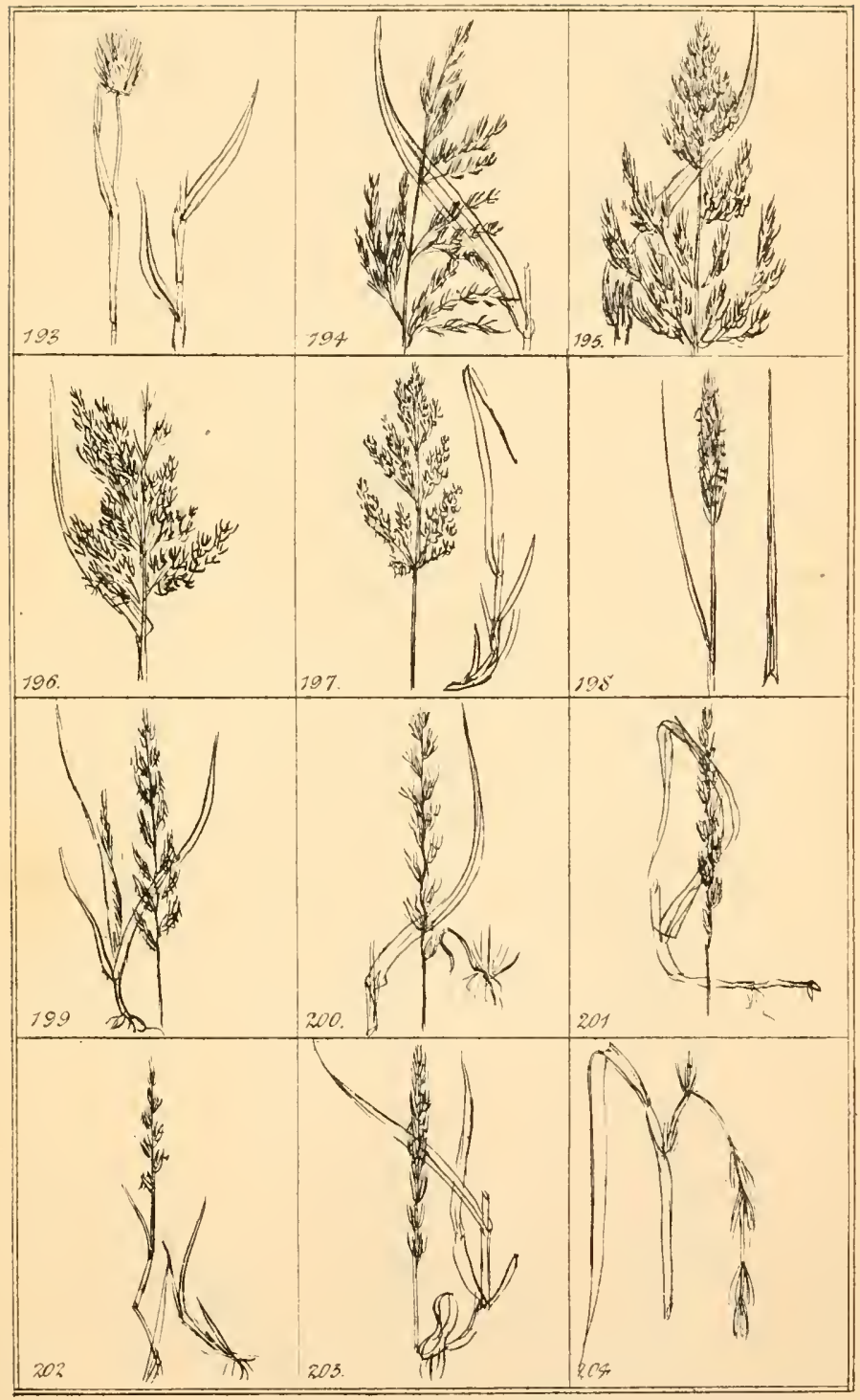





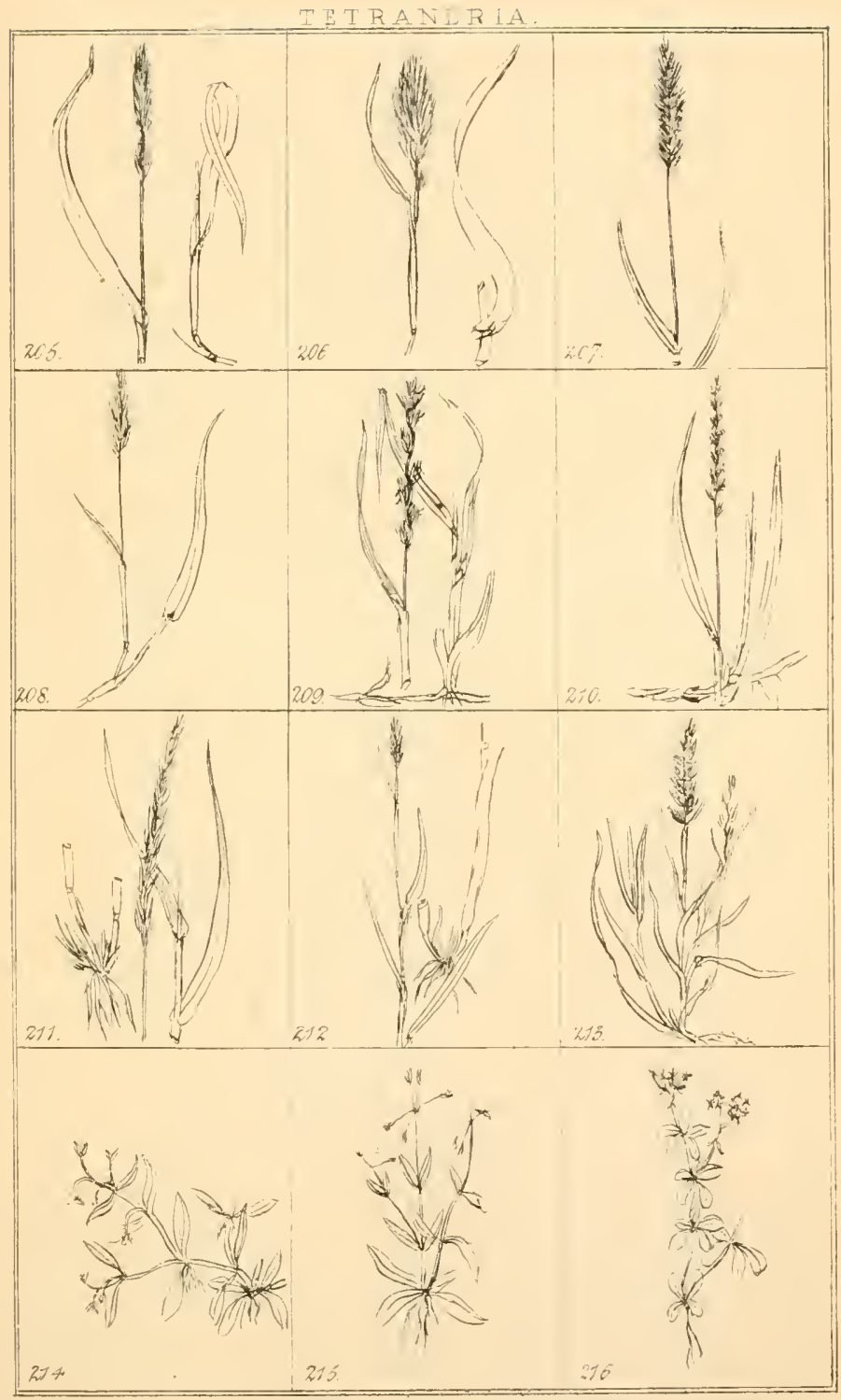





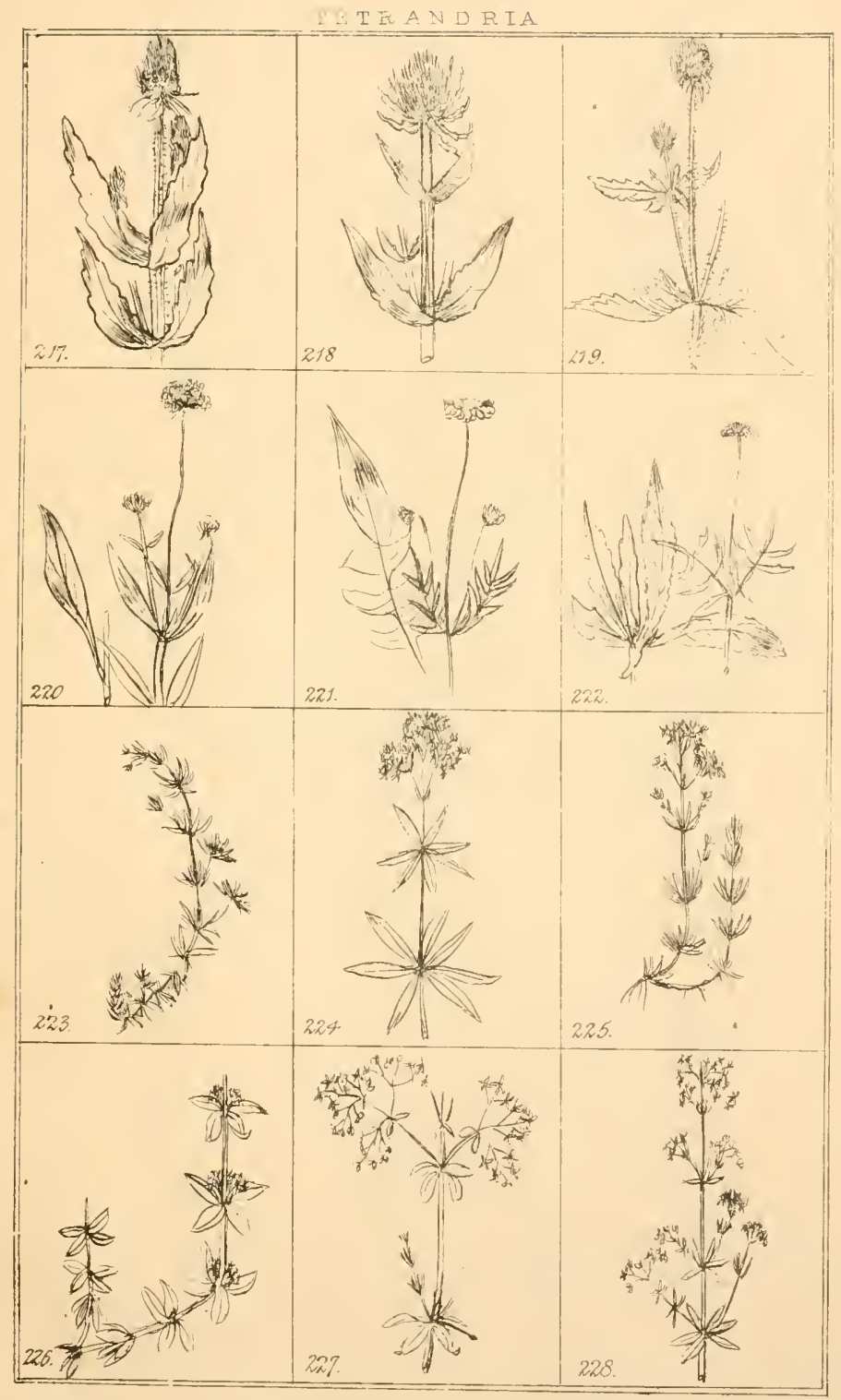



IEIRANDRIA

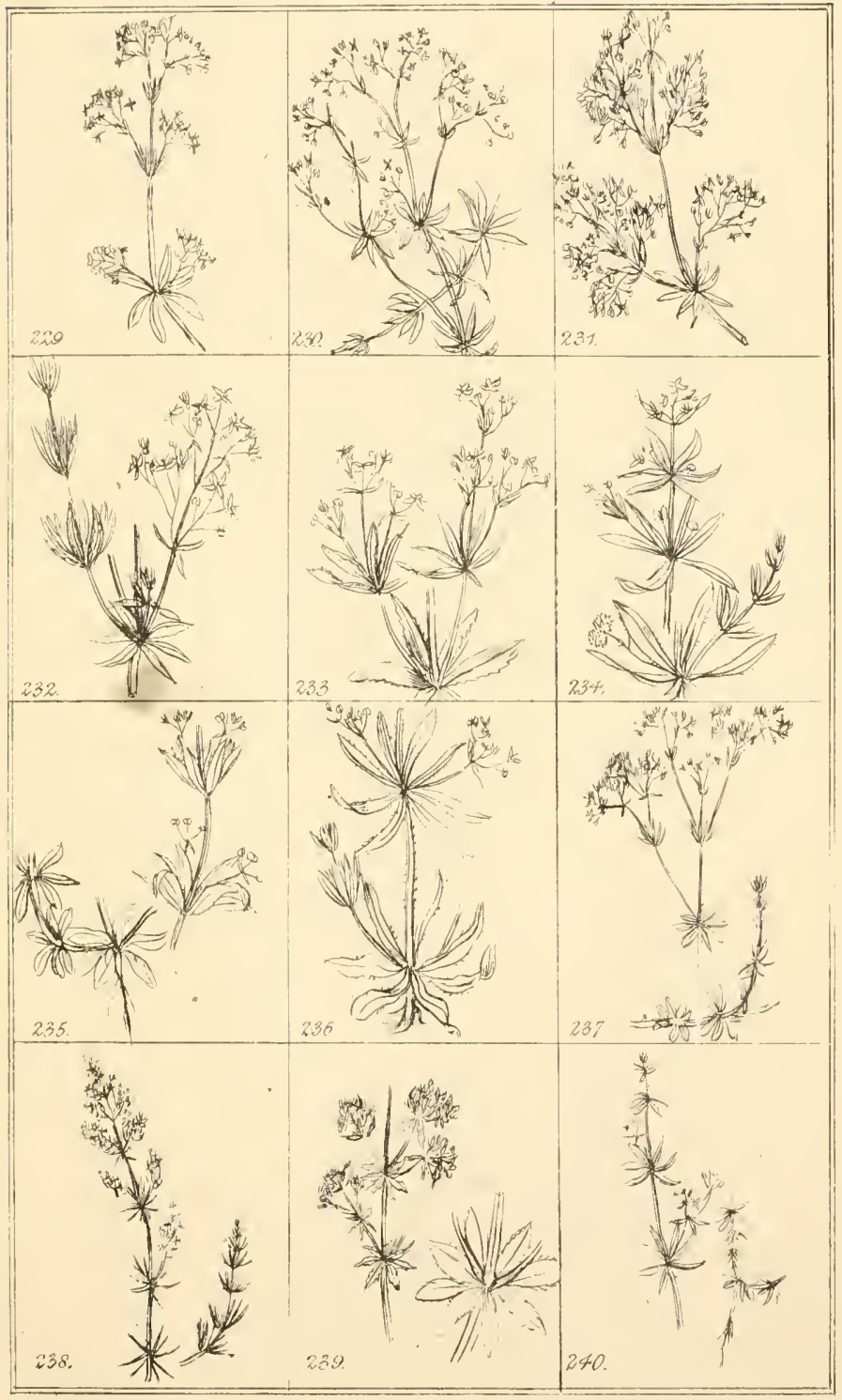





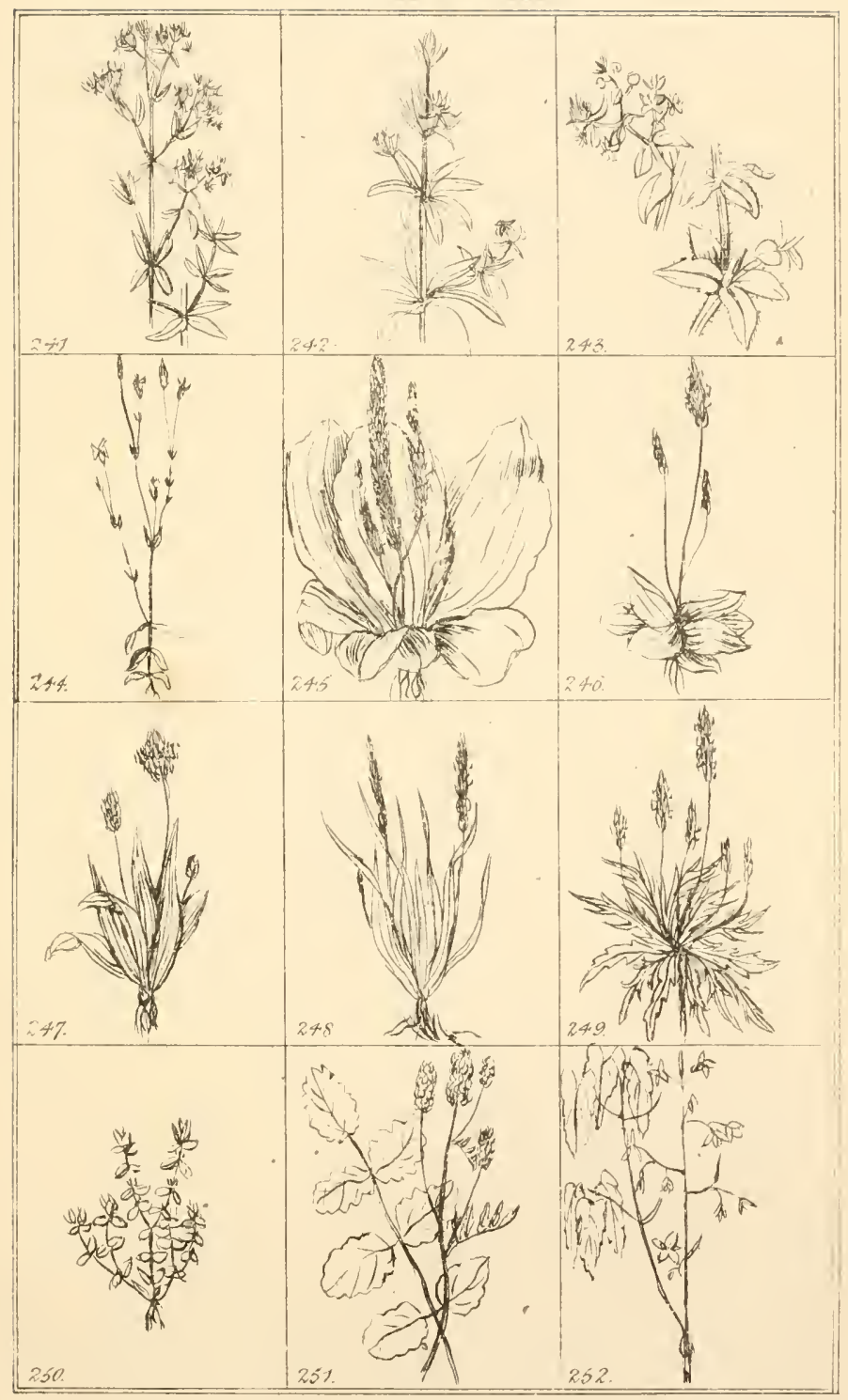



TEIRANDRIA

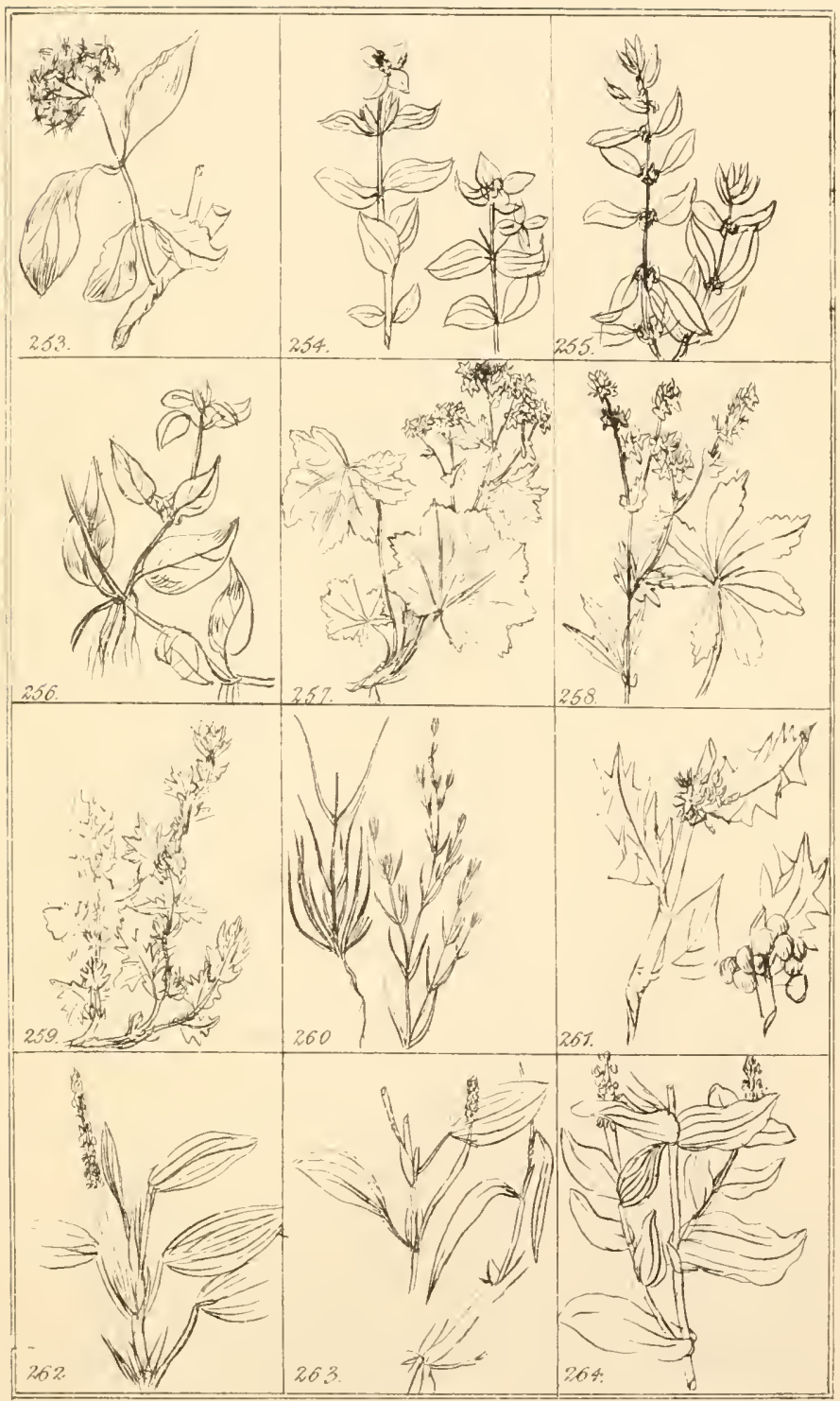





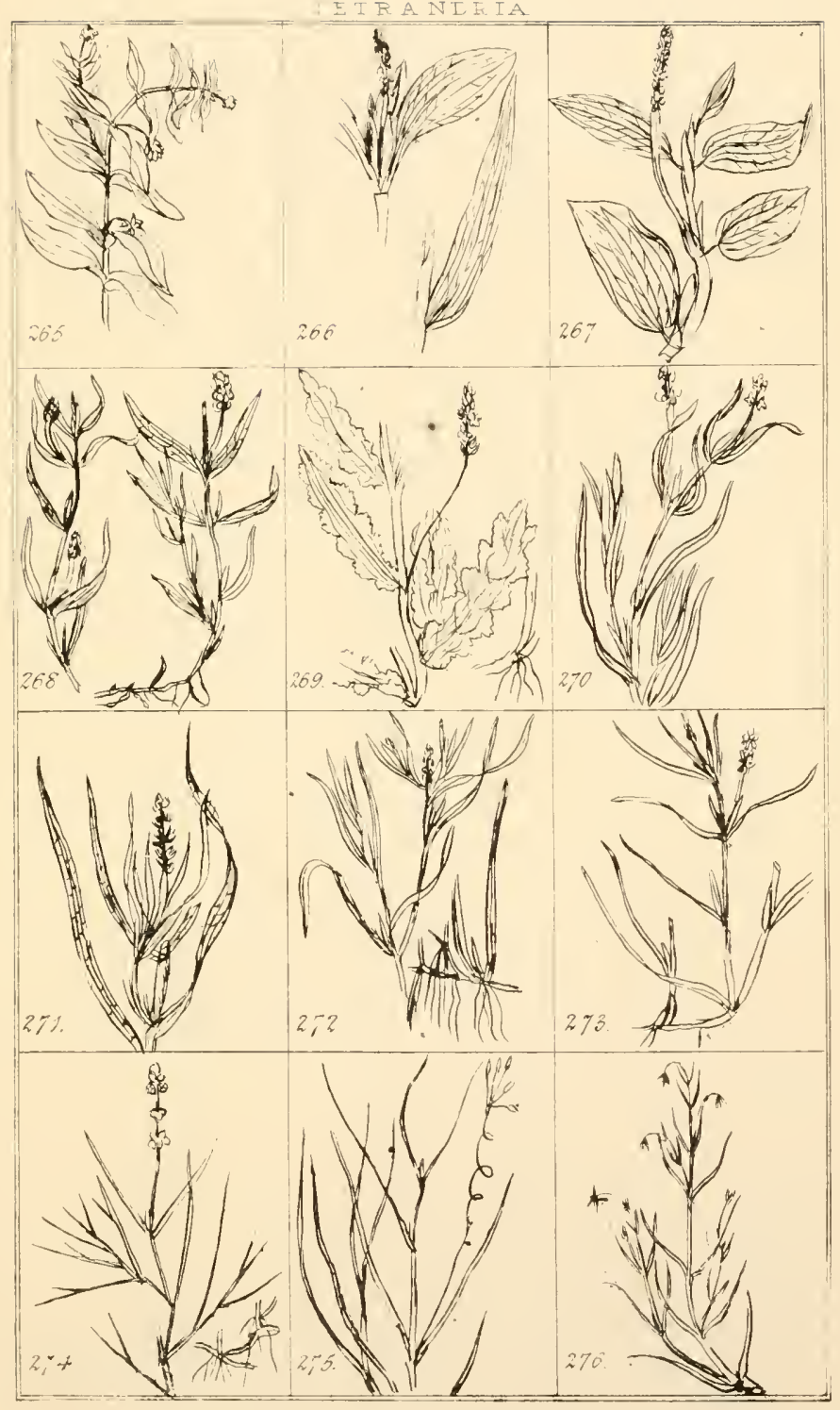





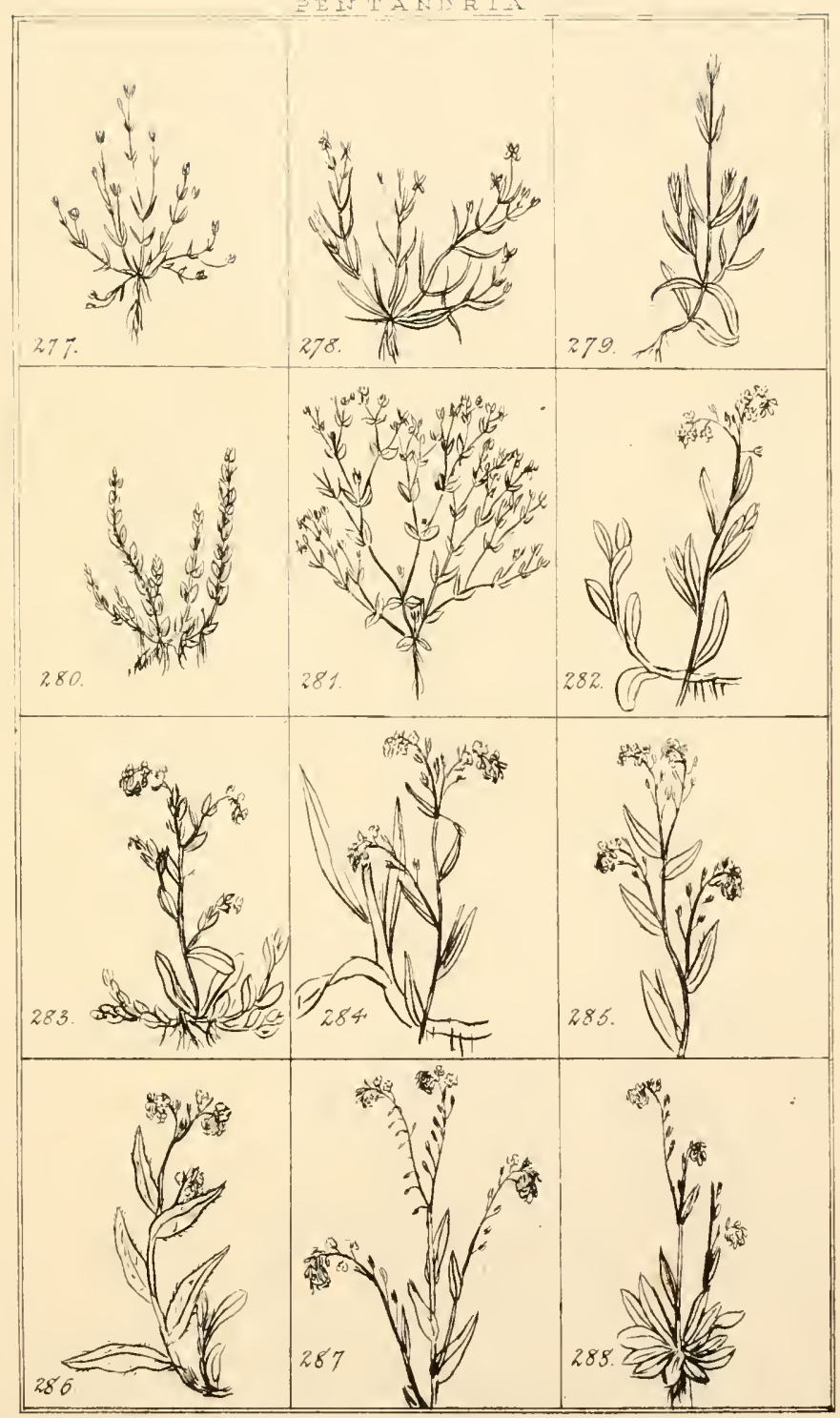



PENTADE

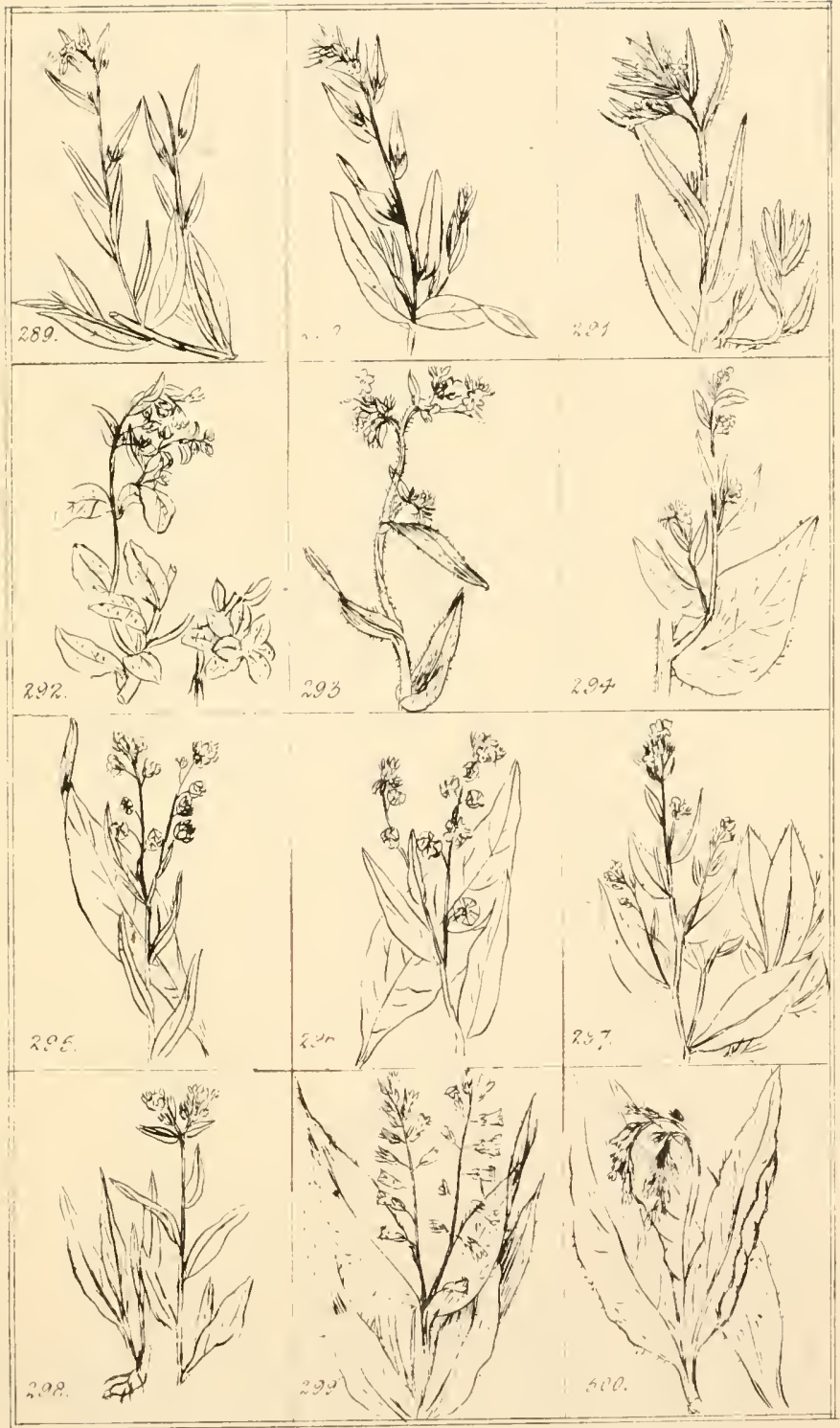



LEI, I \& N L R I

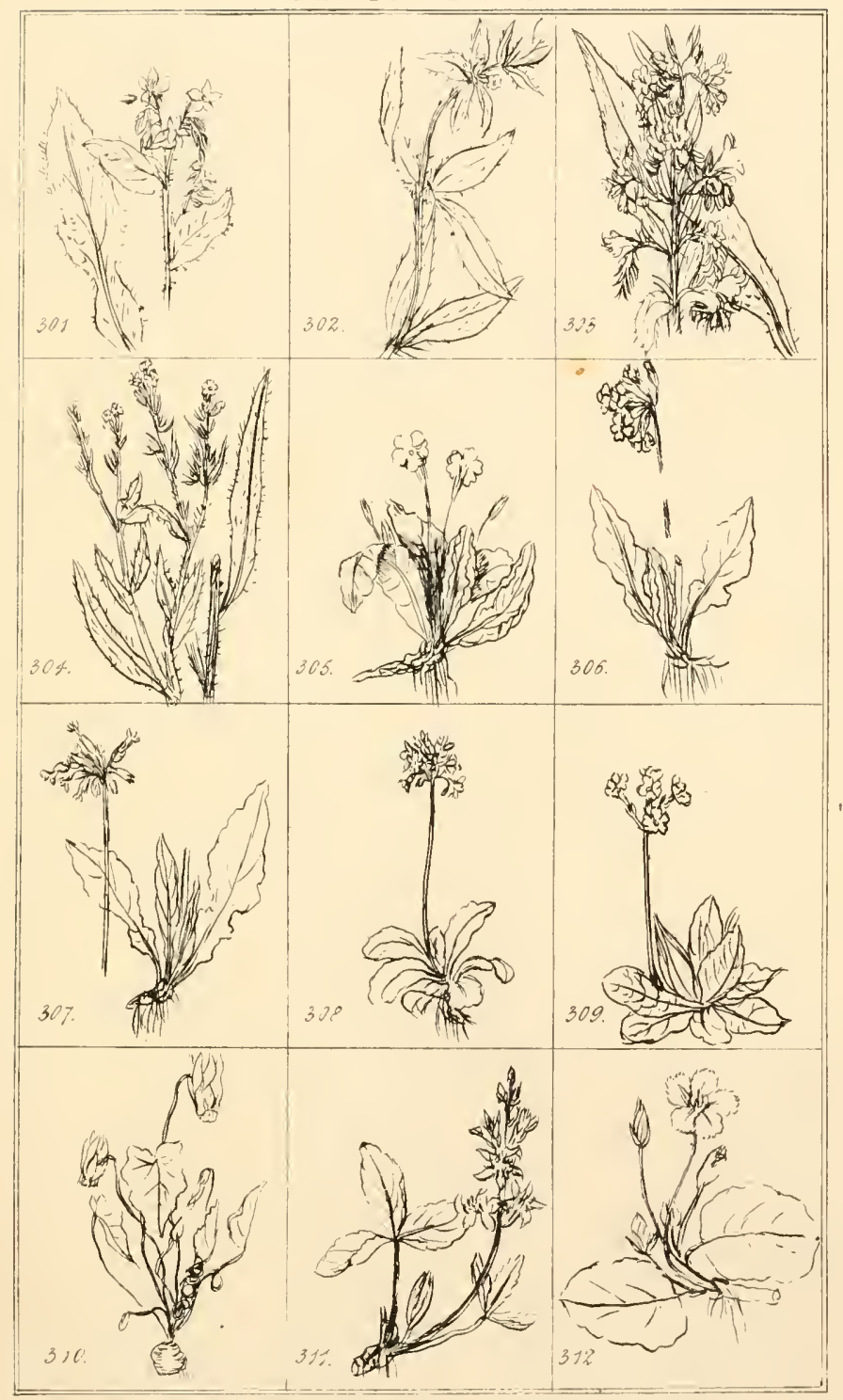





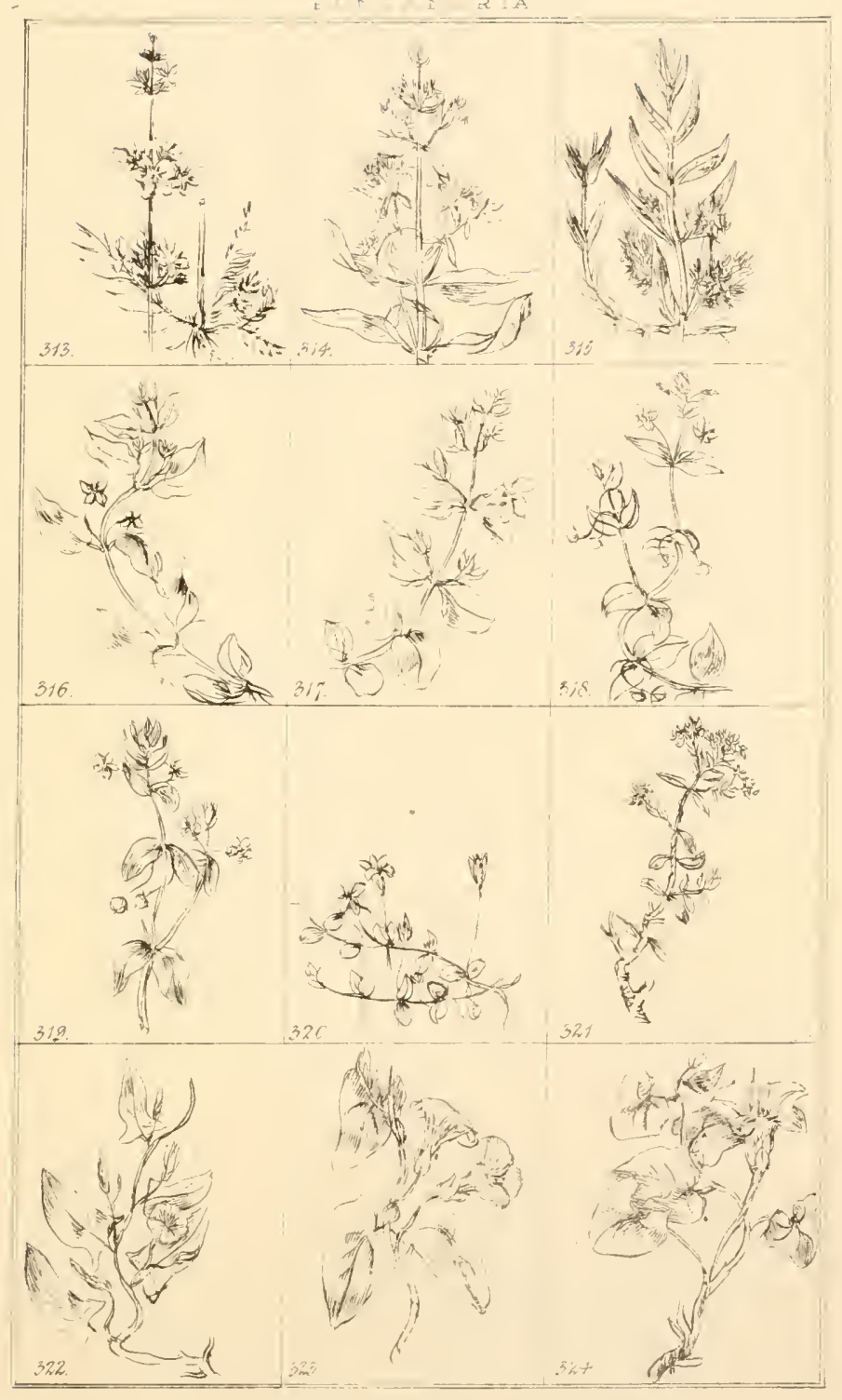





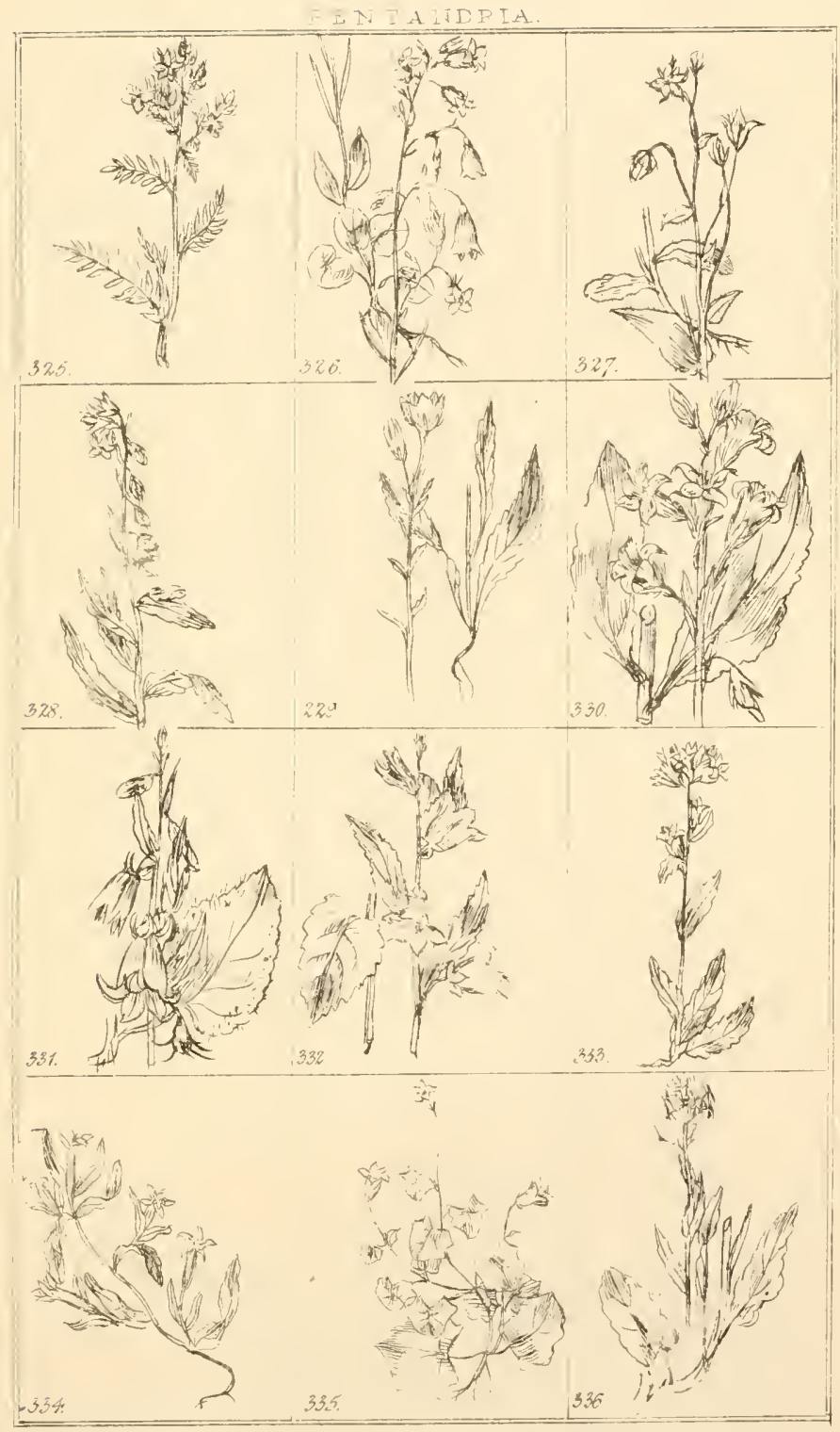





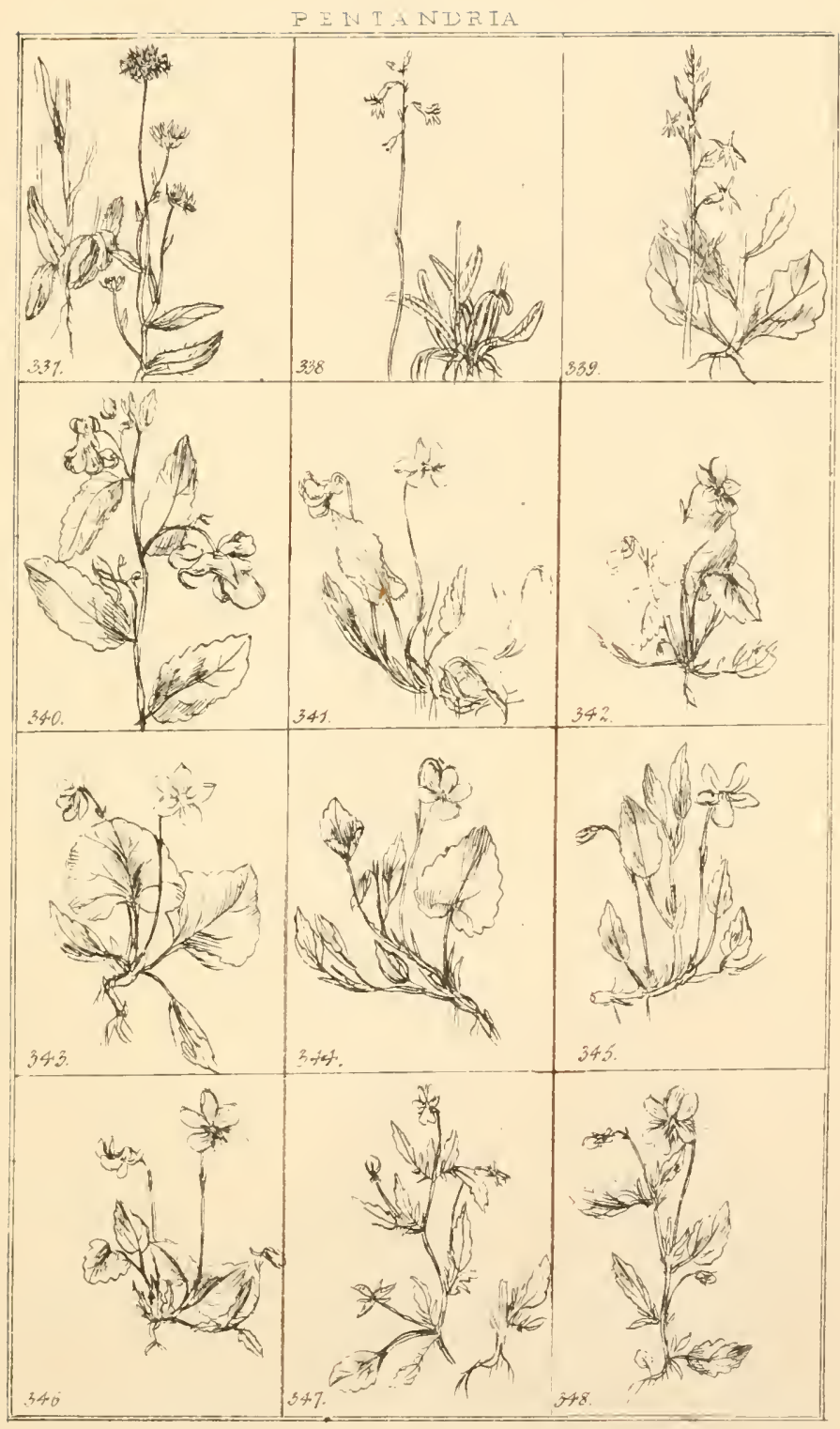





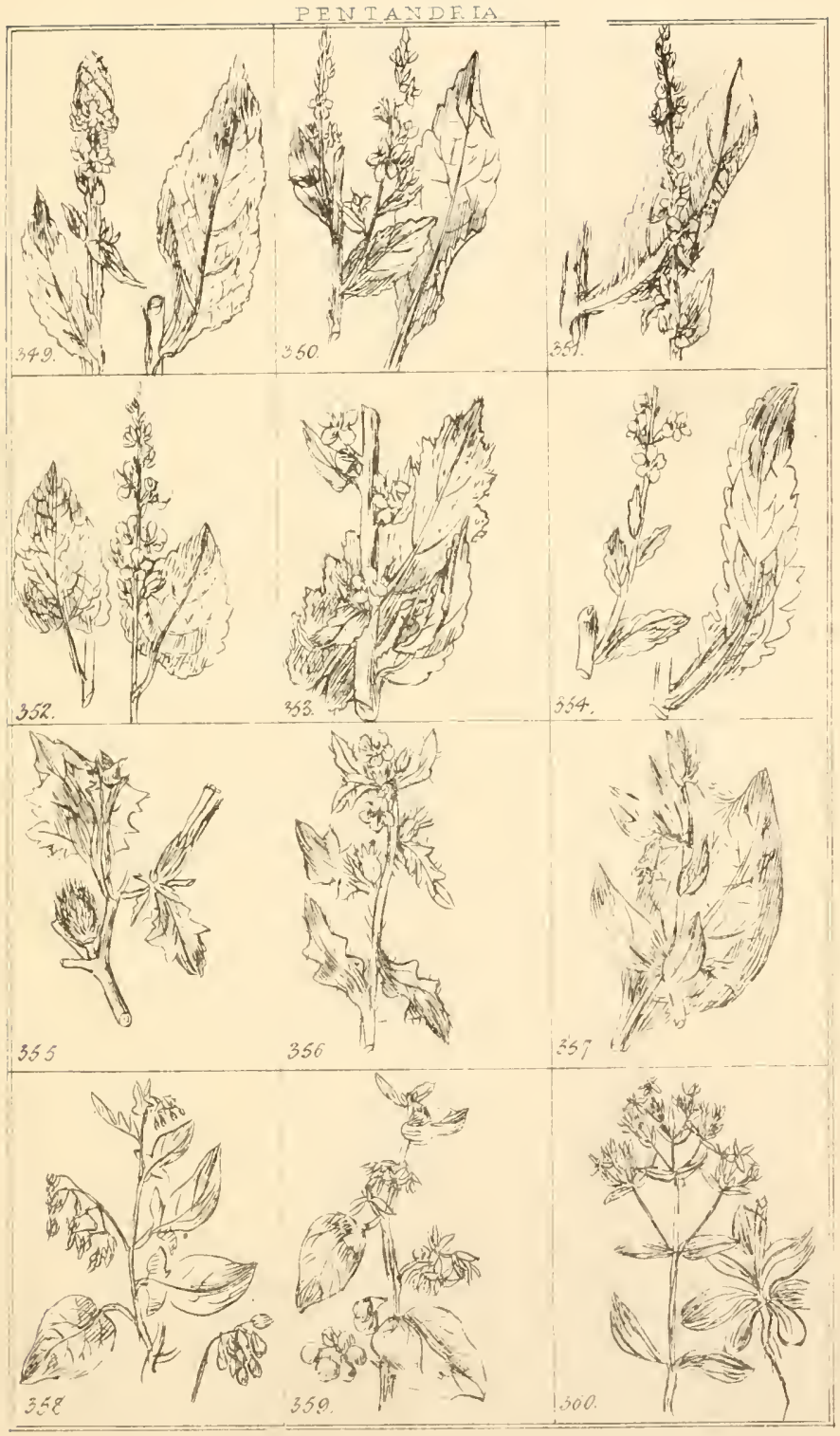



PENTANDRIA

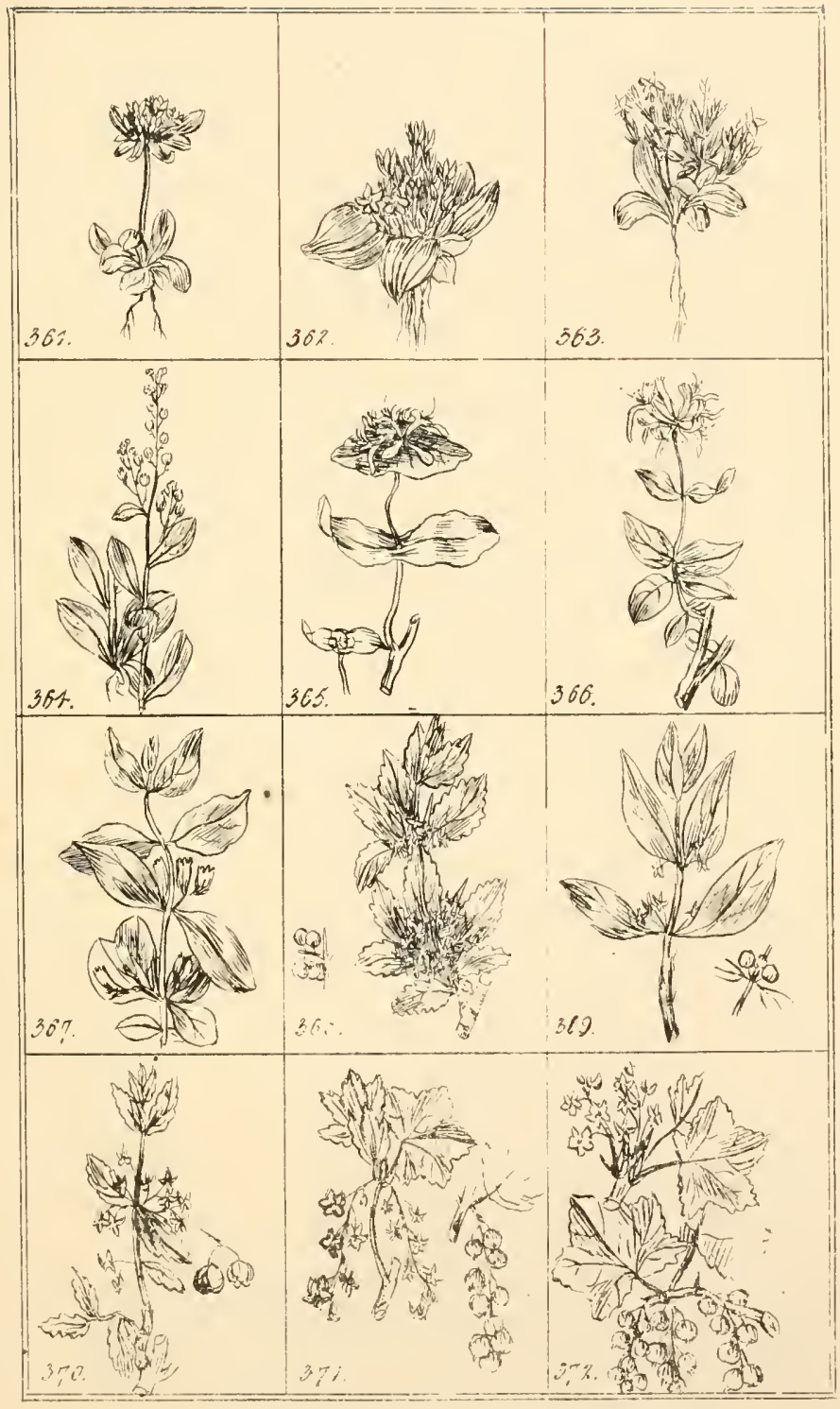





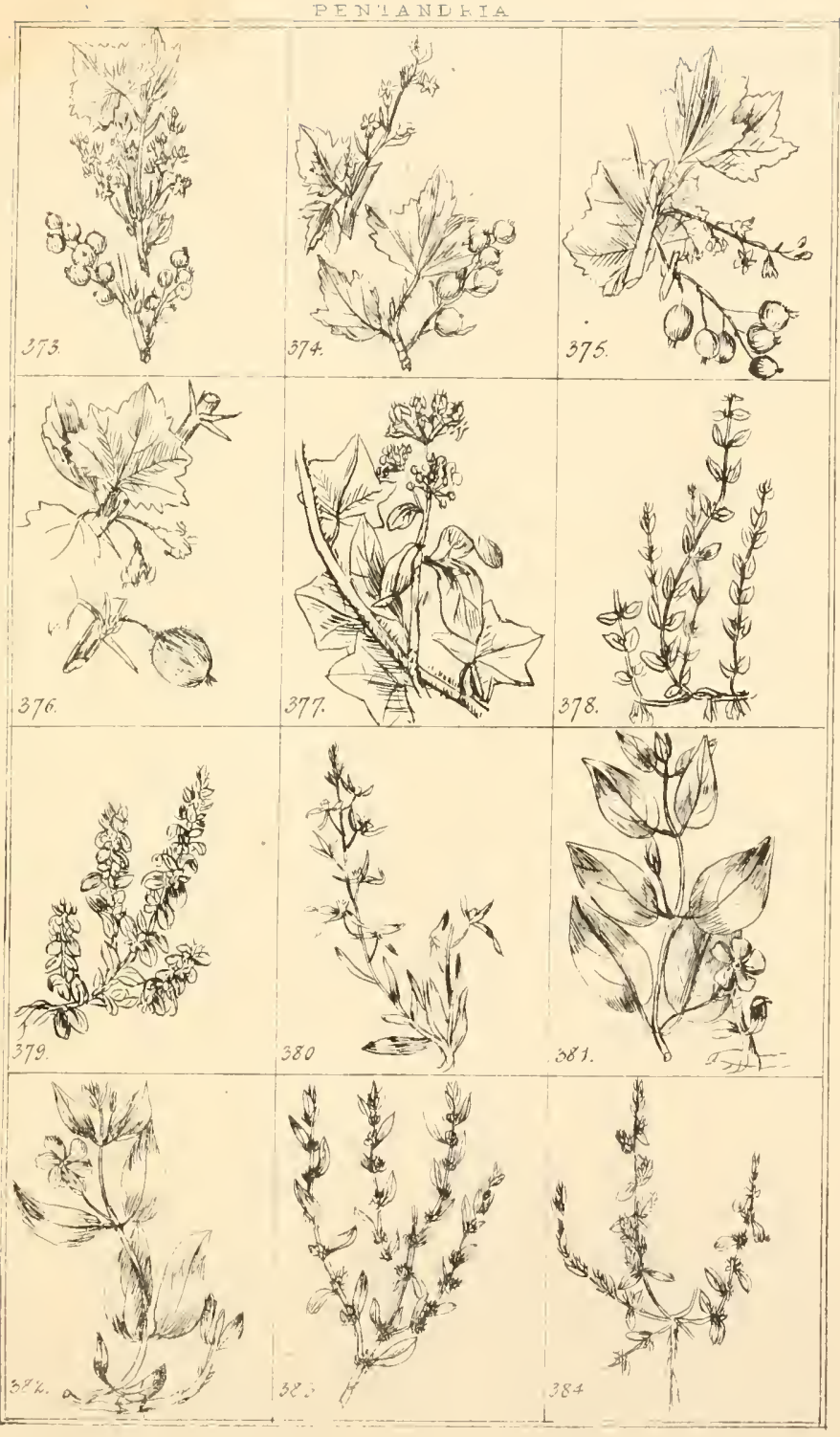





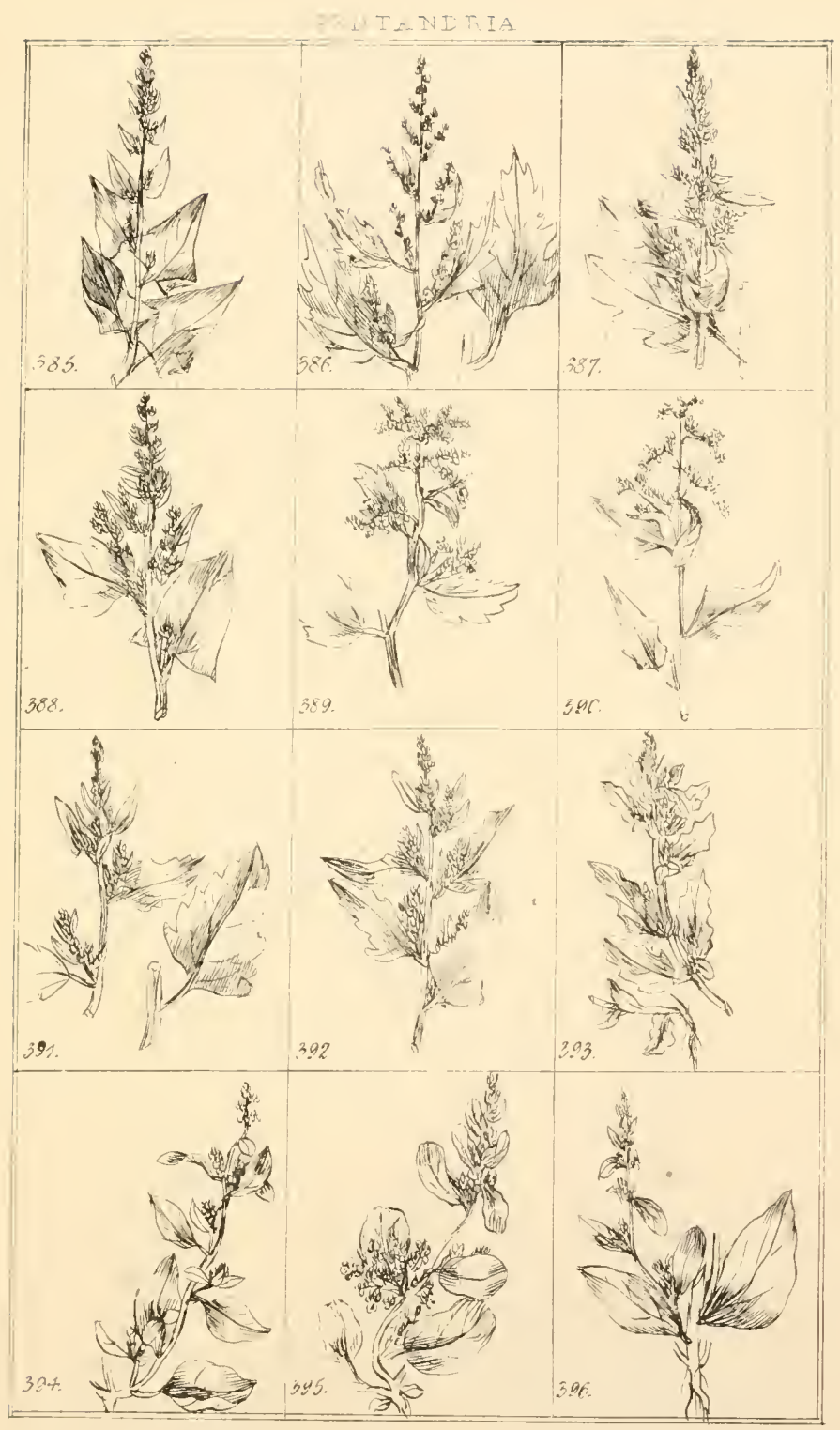





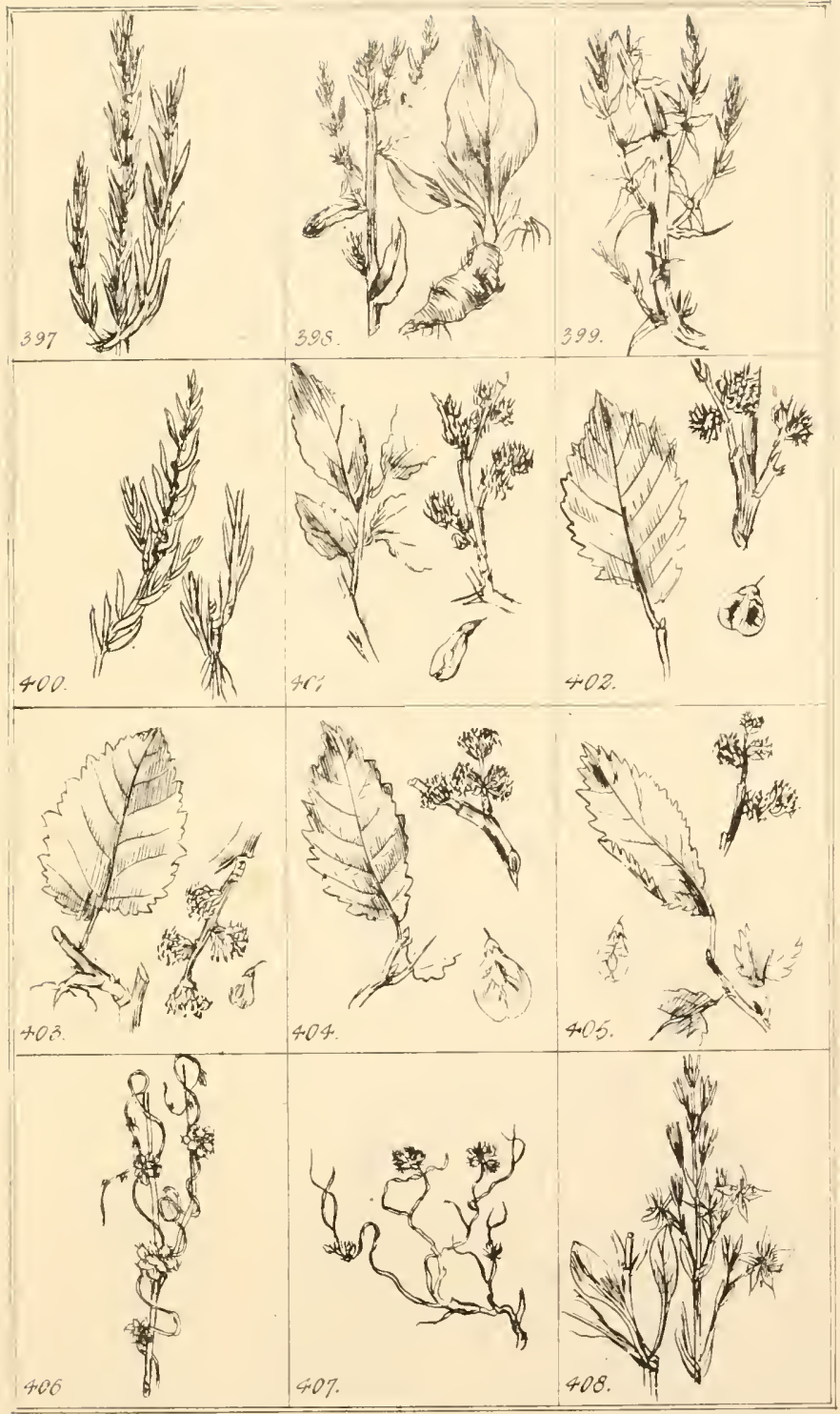



PENT $+N D R$ I A

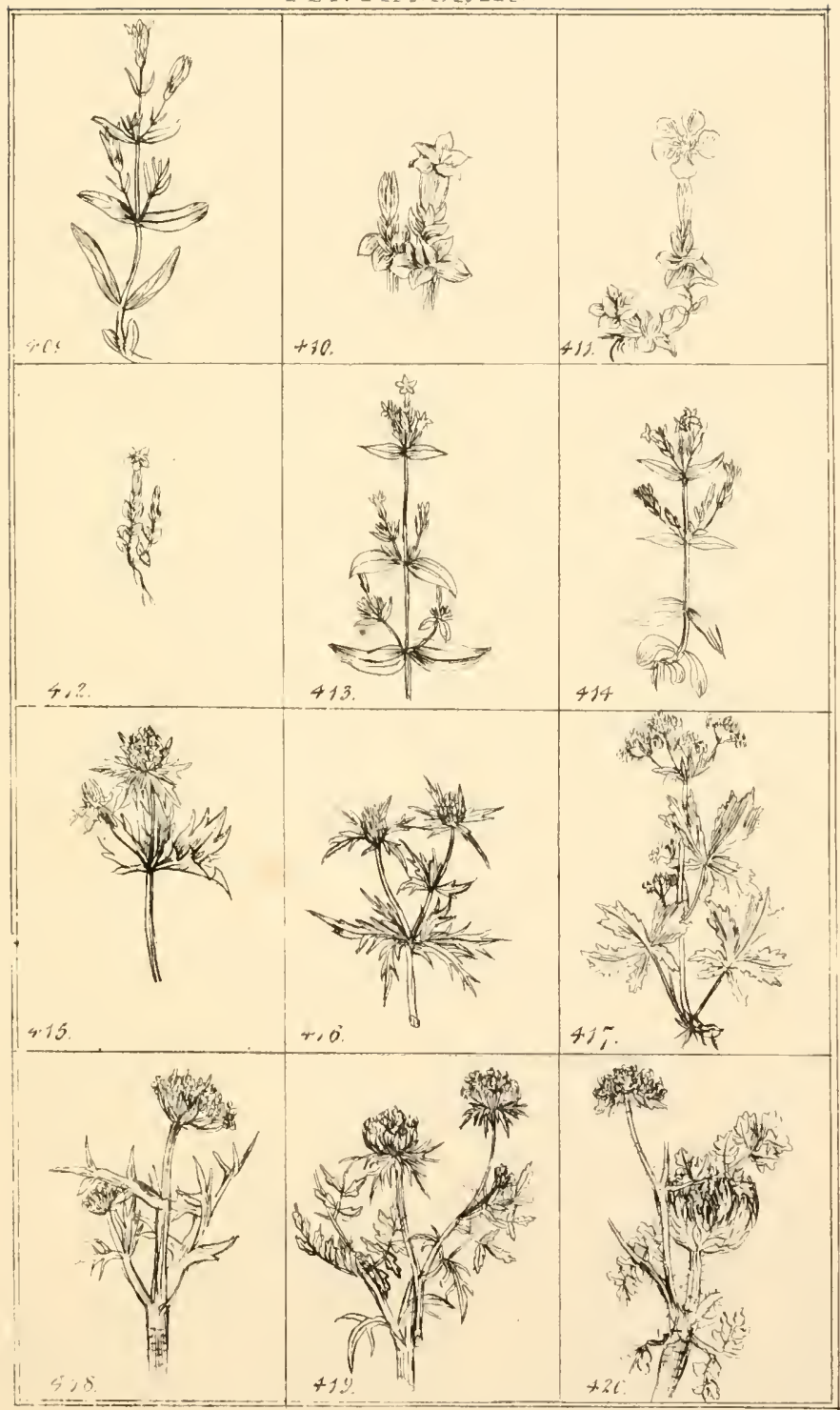



PENTAIIDIIA

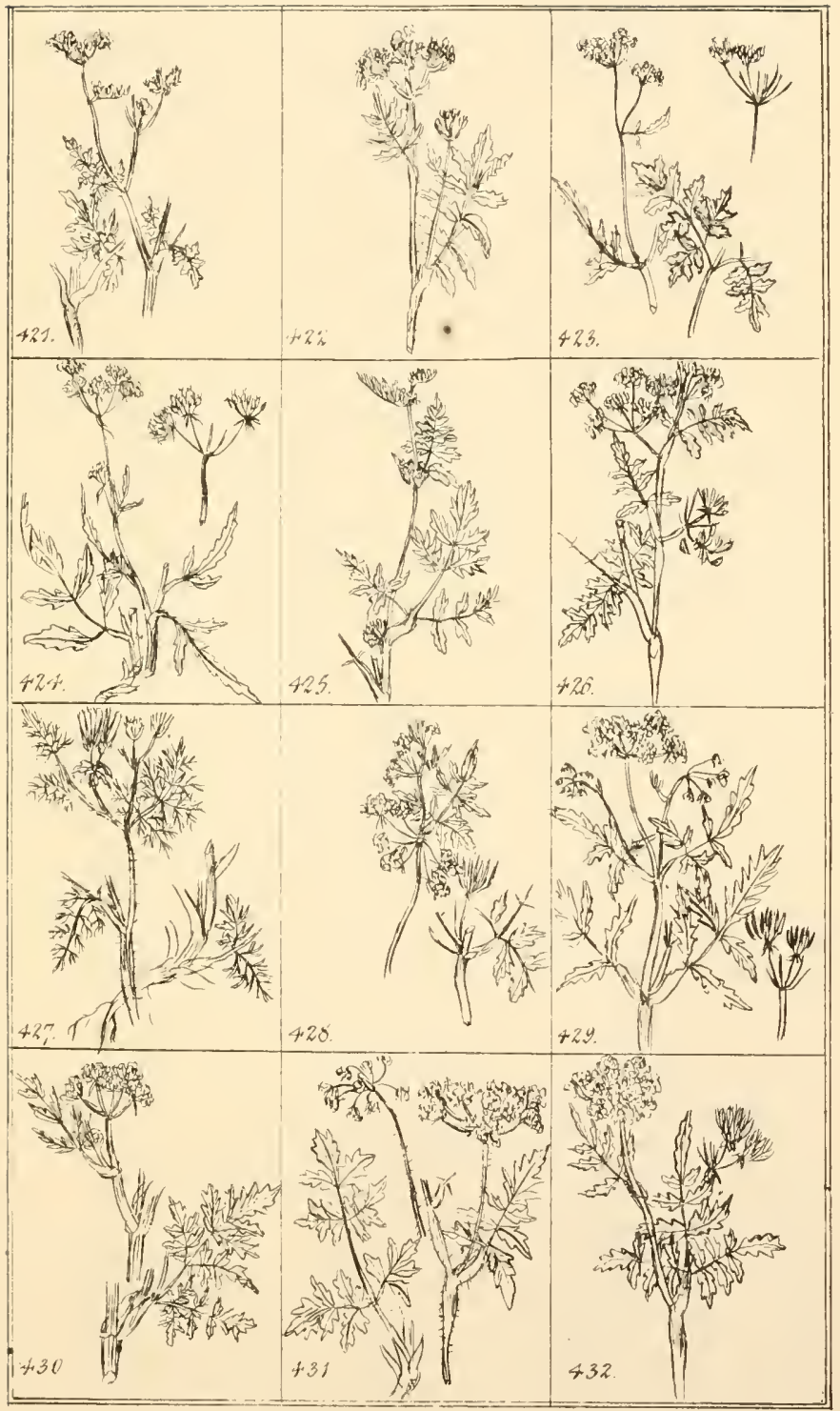





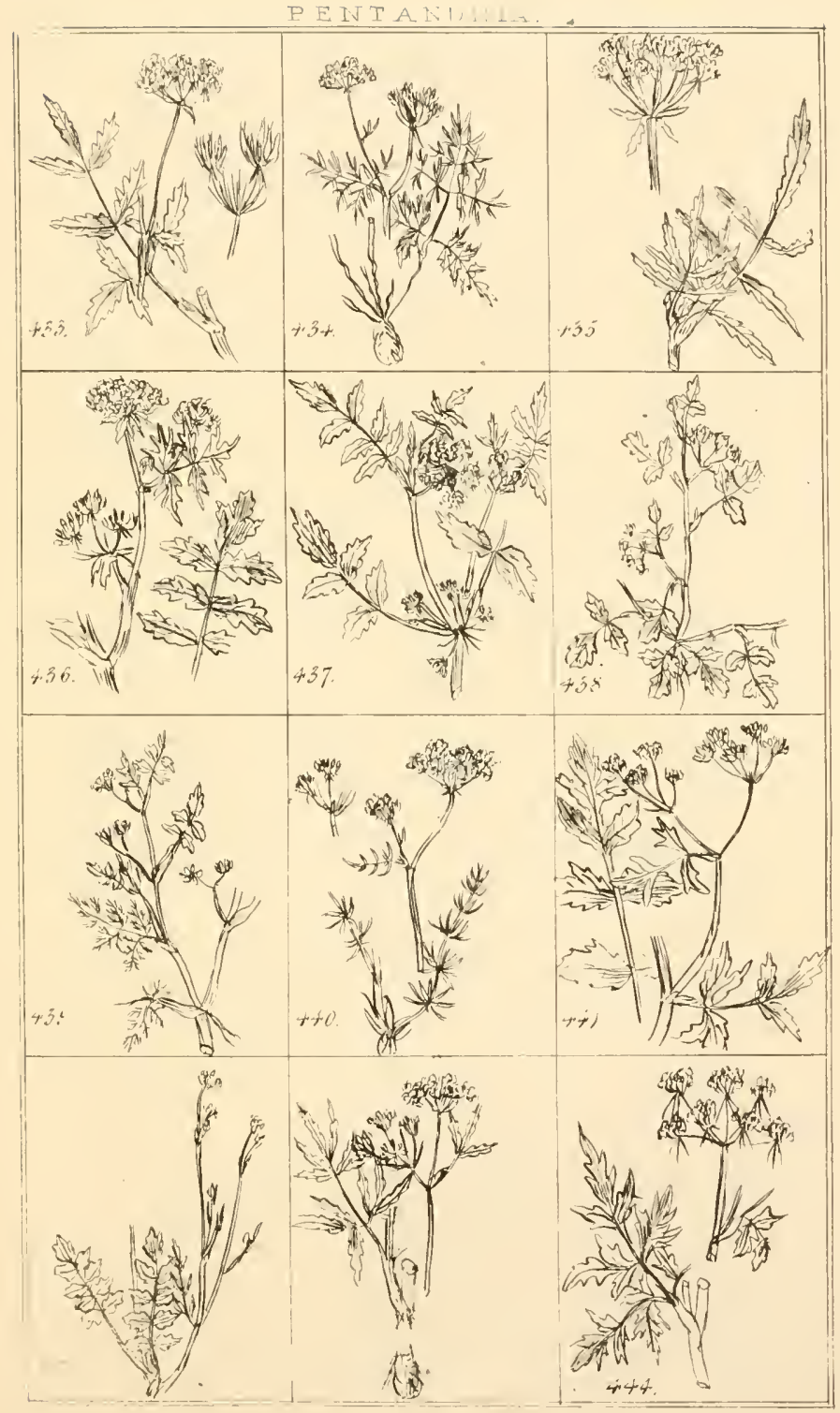





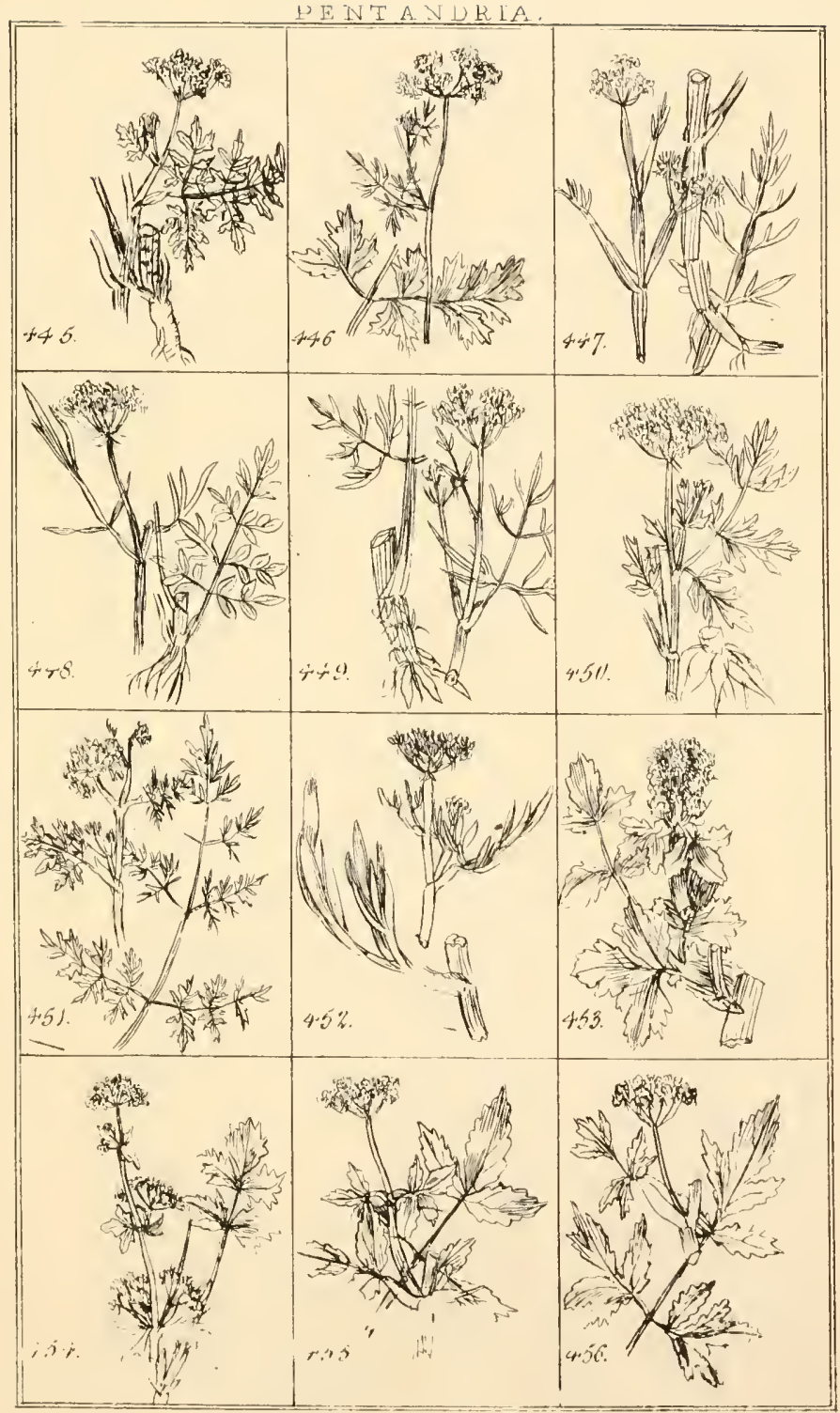



PENTANDEIA

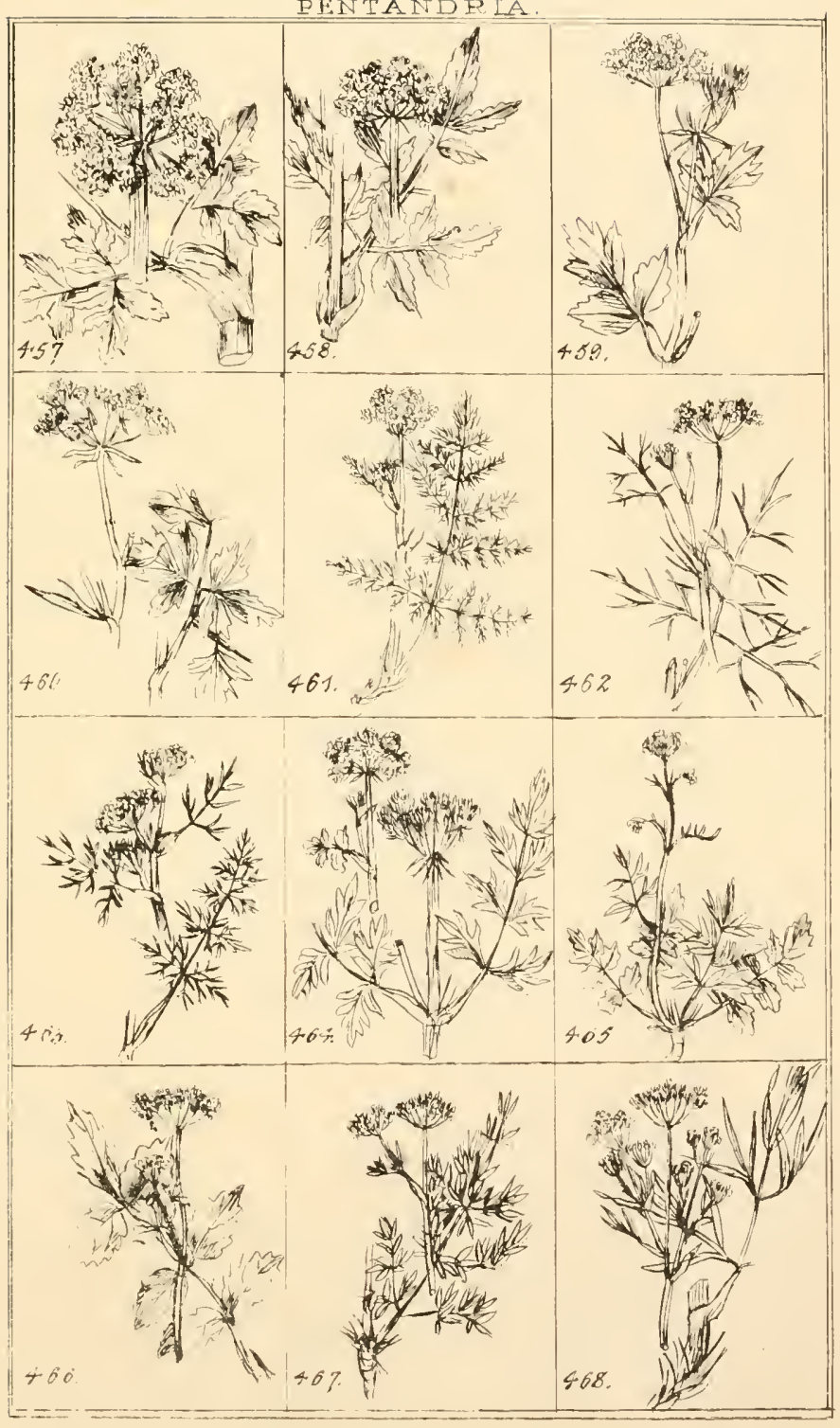



PENTANDRIA

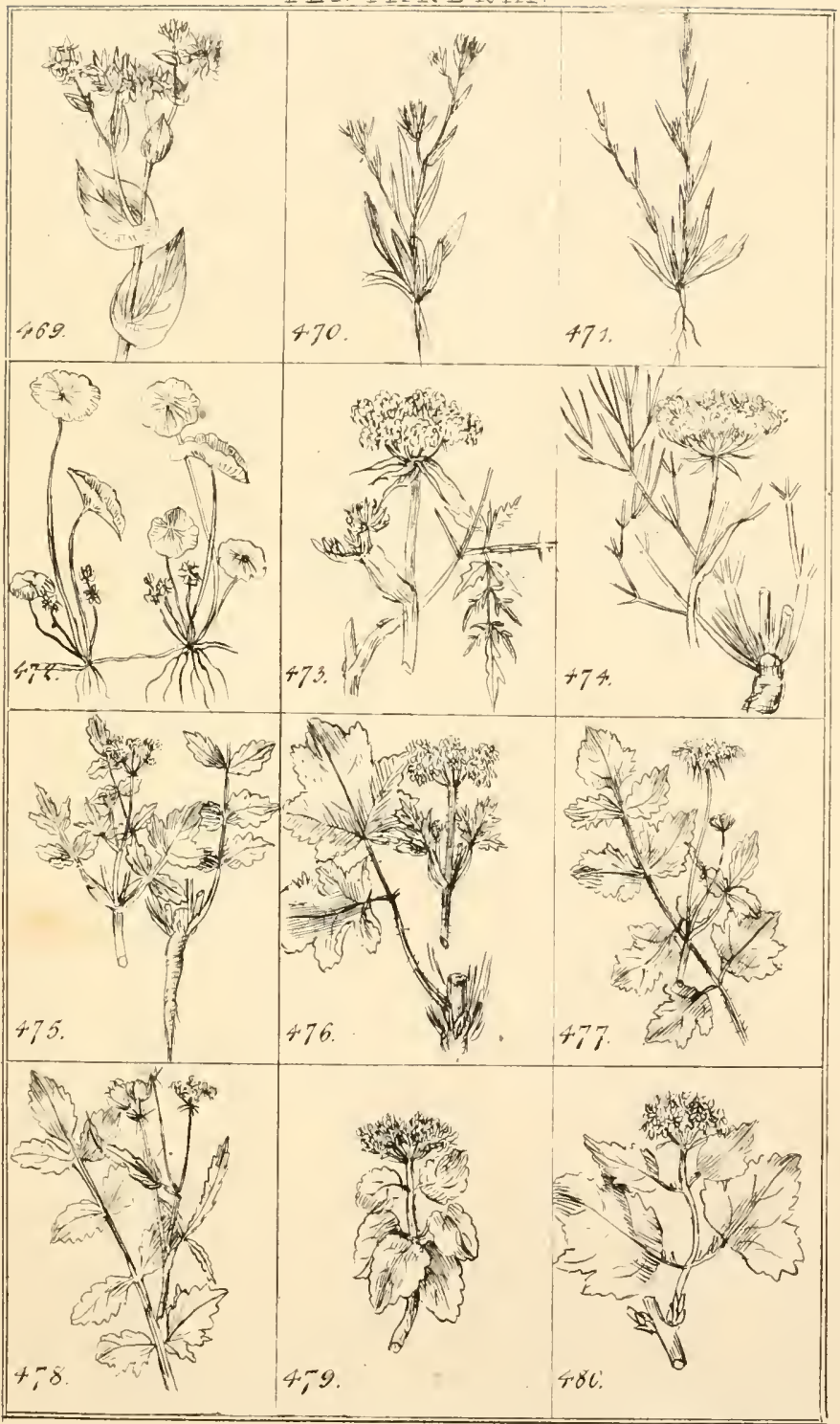



PFIT'TANDRIA.

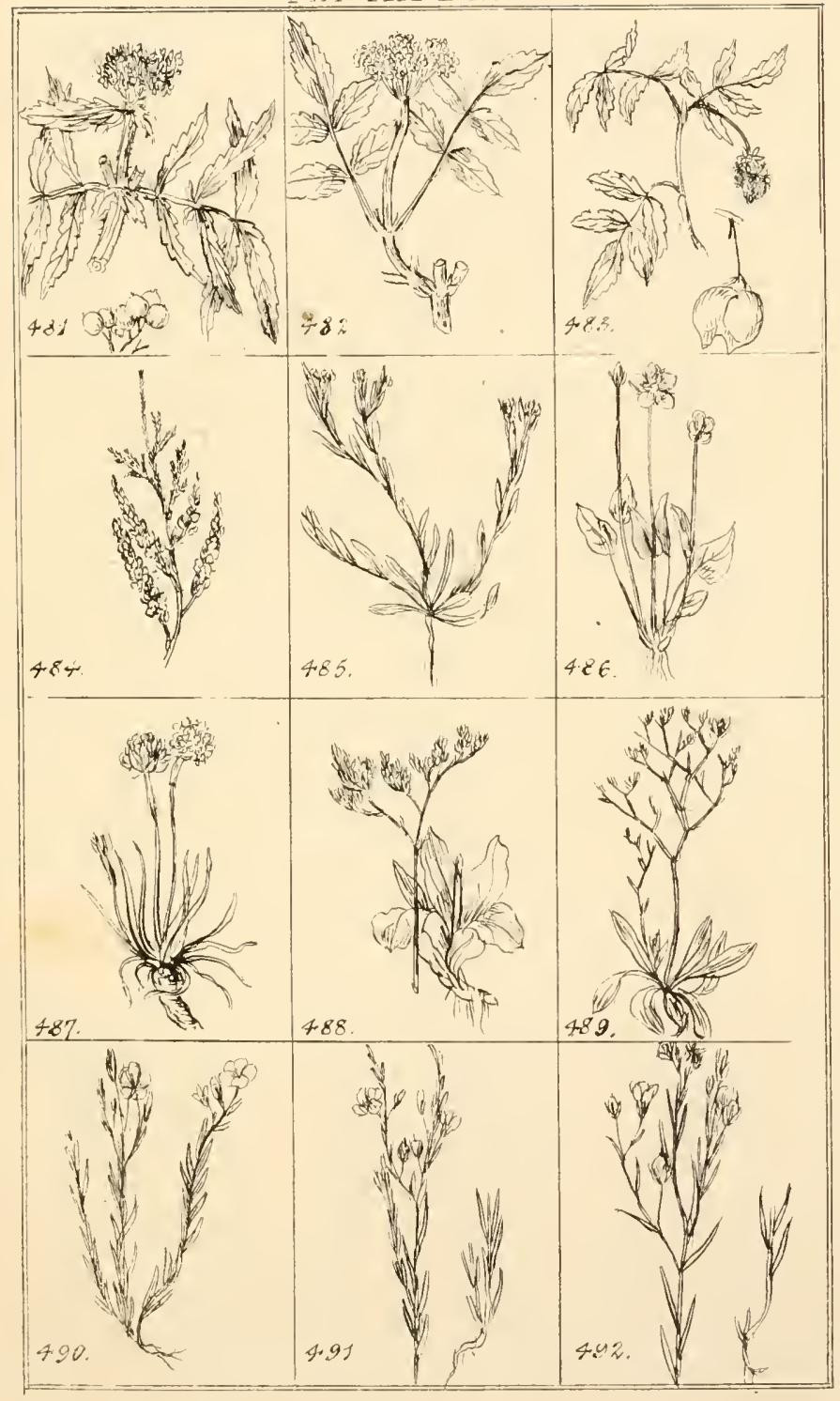





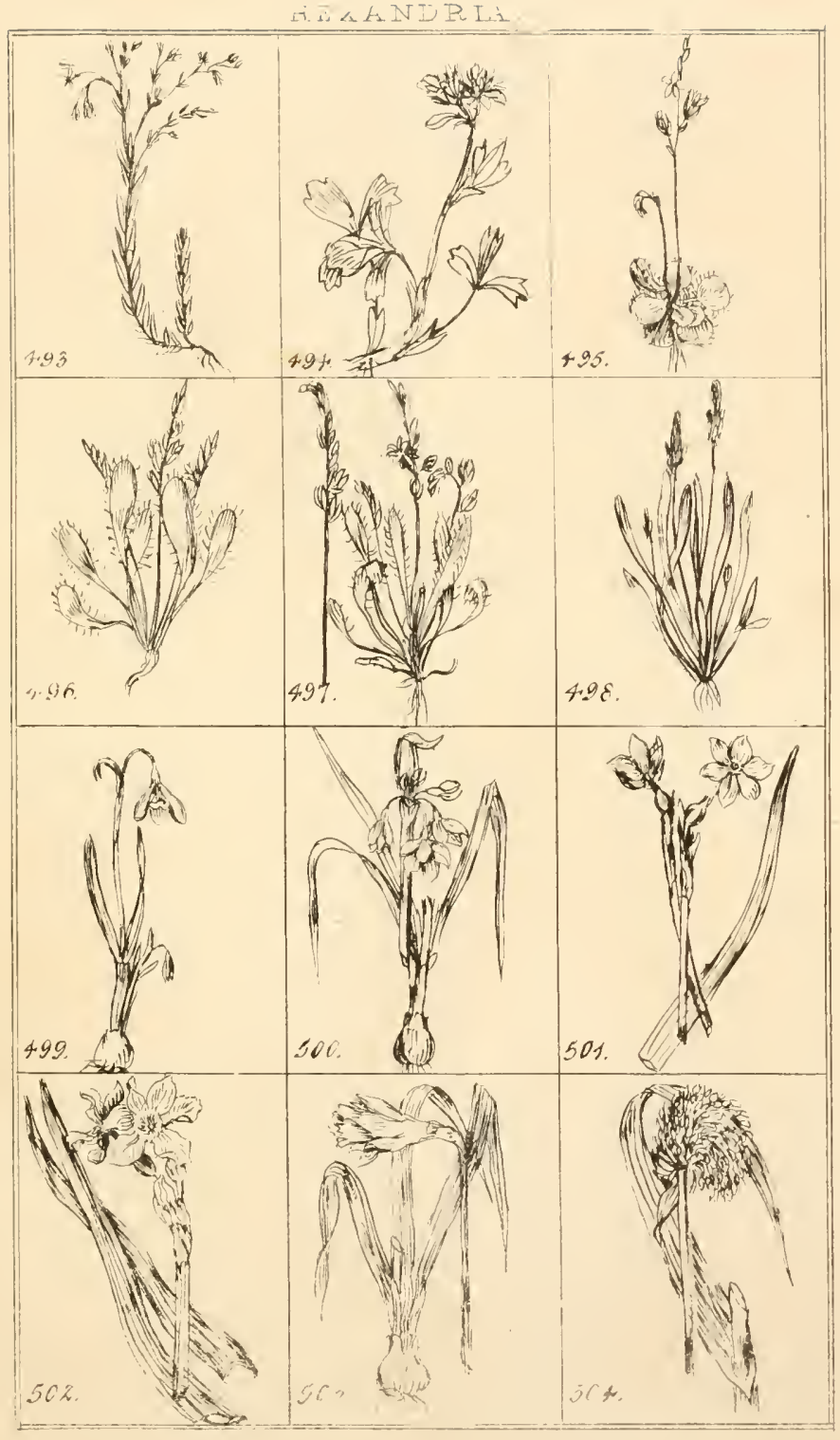





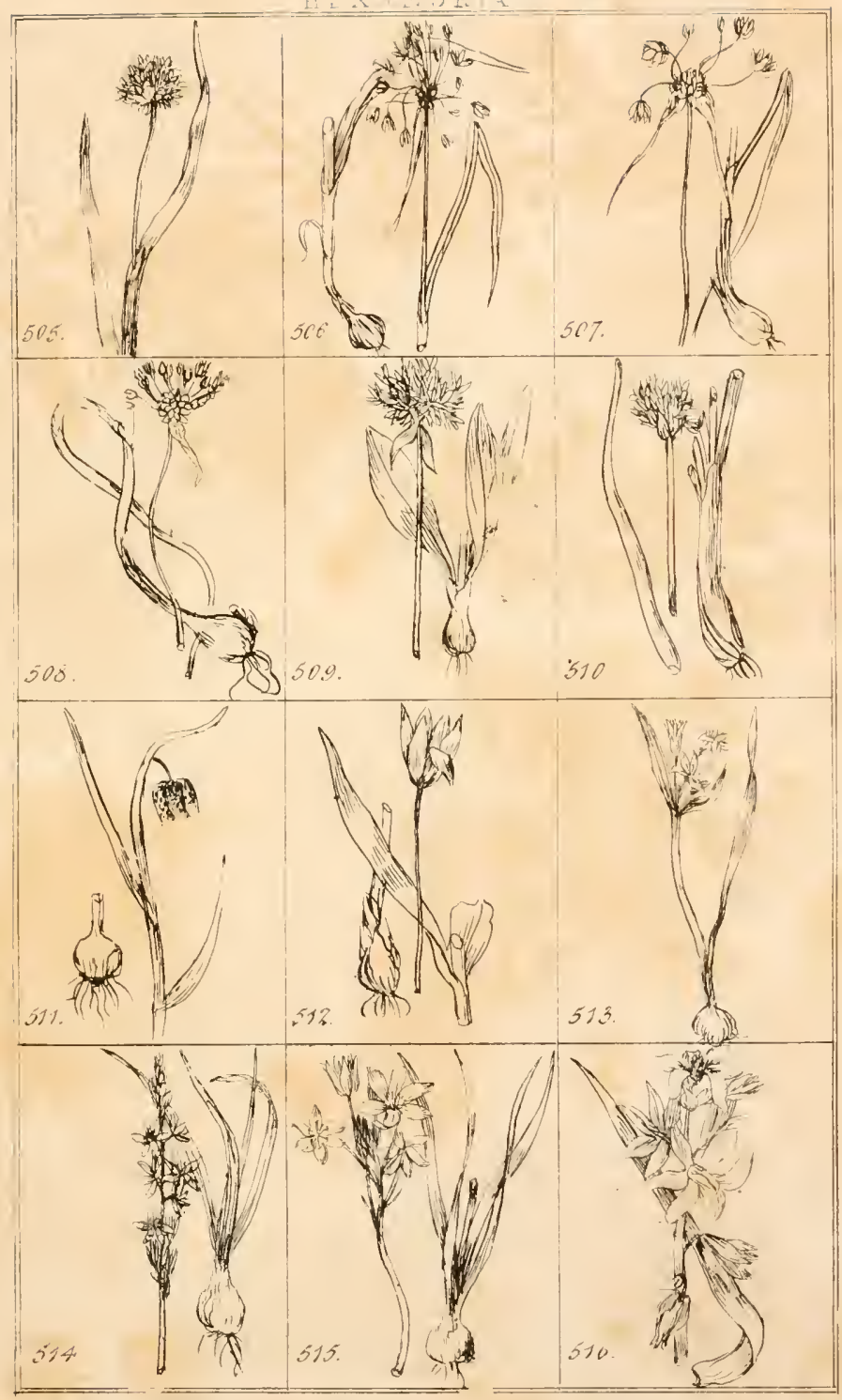




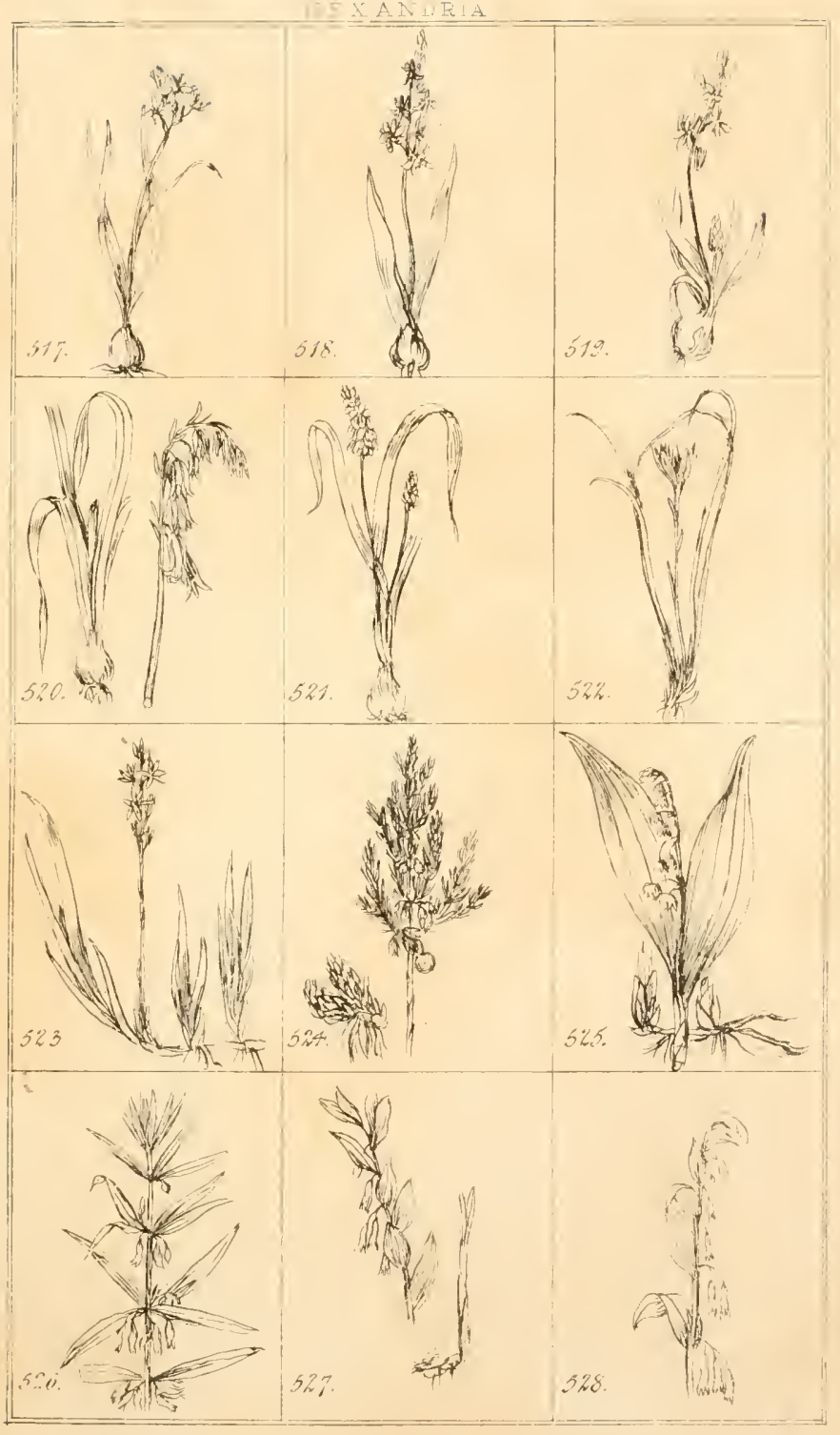





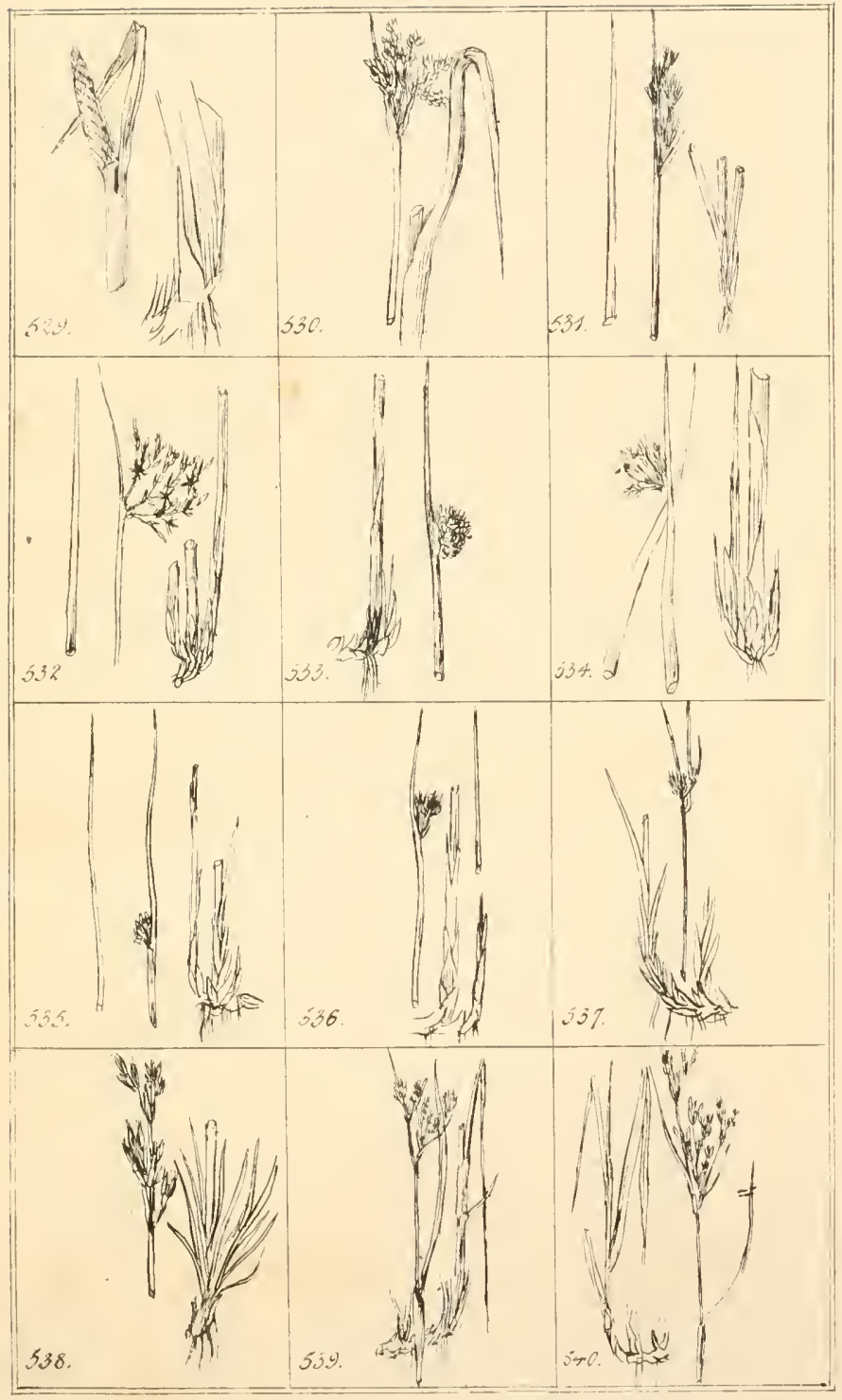





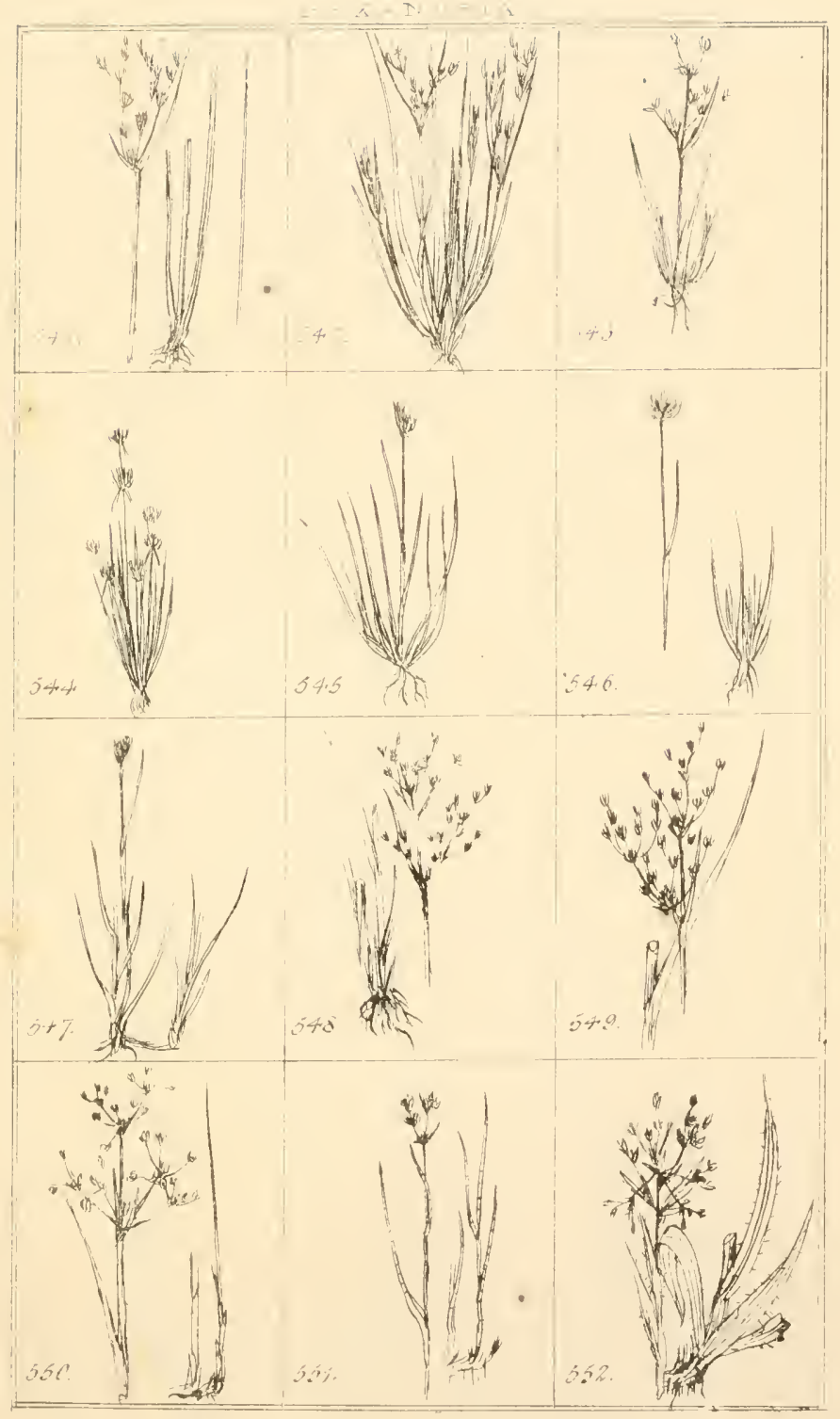



$1 E K$ \& 14 D F 1

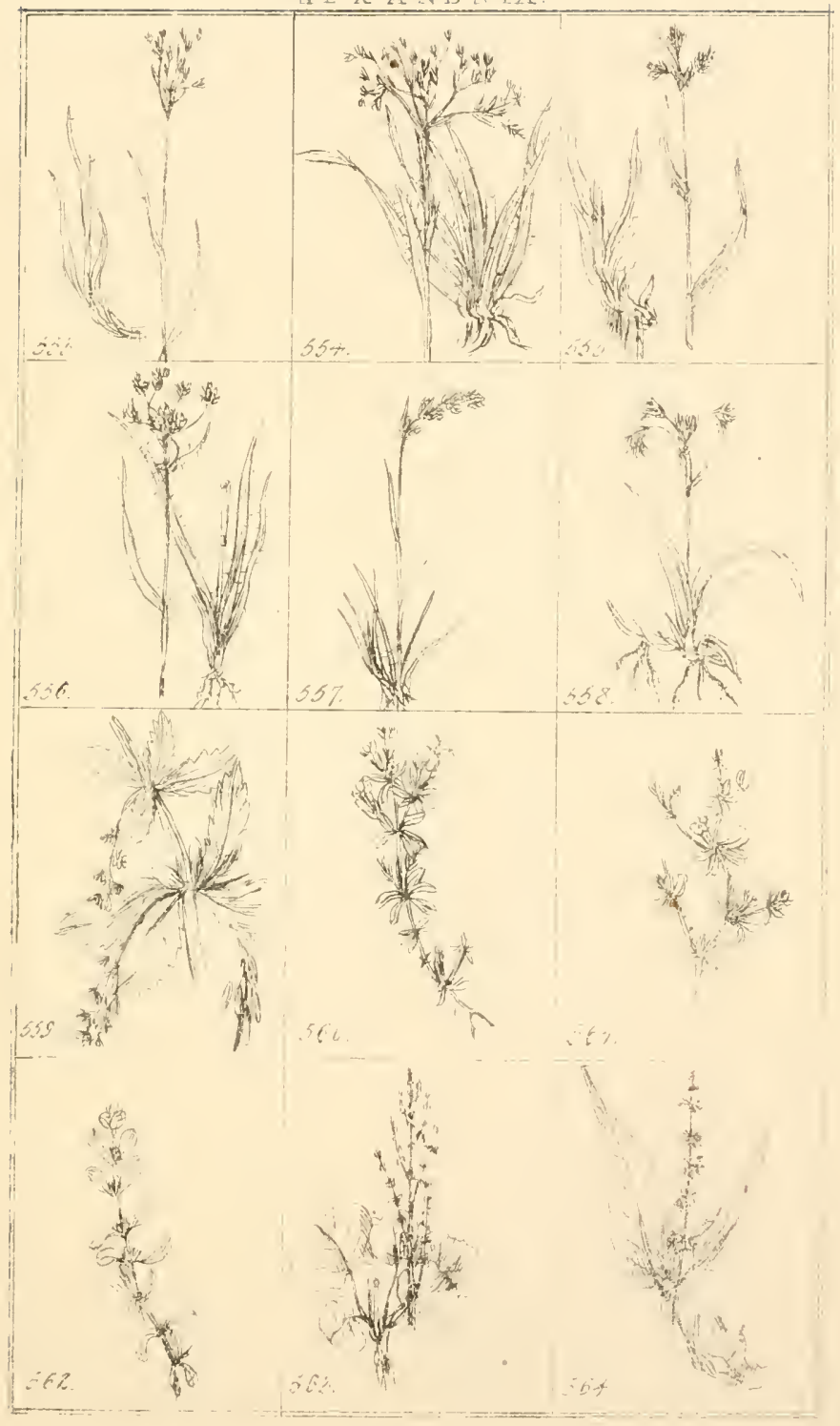





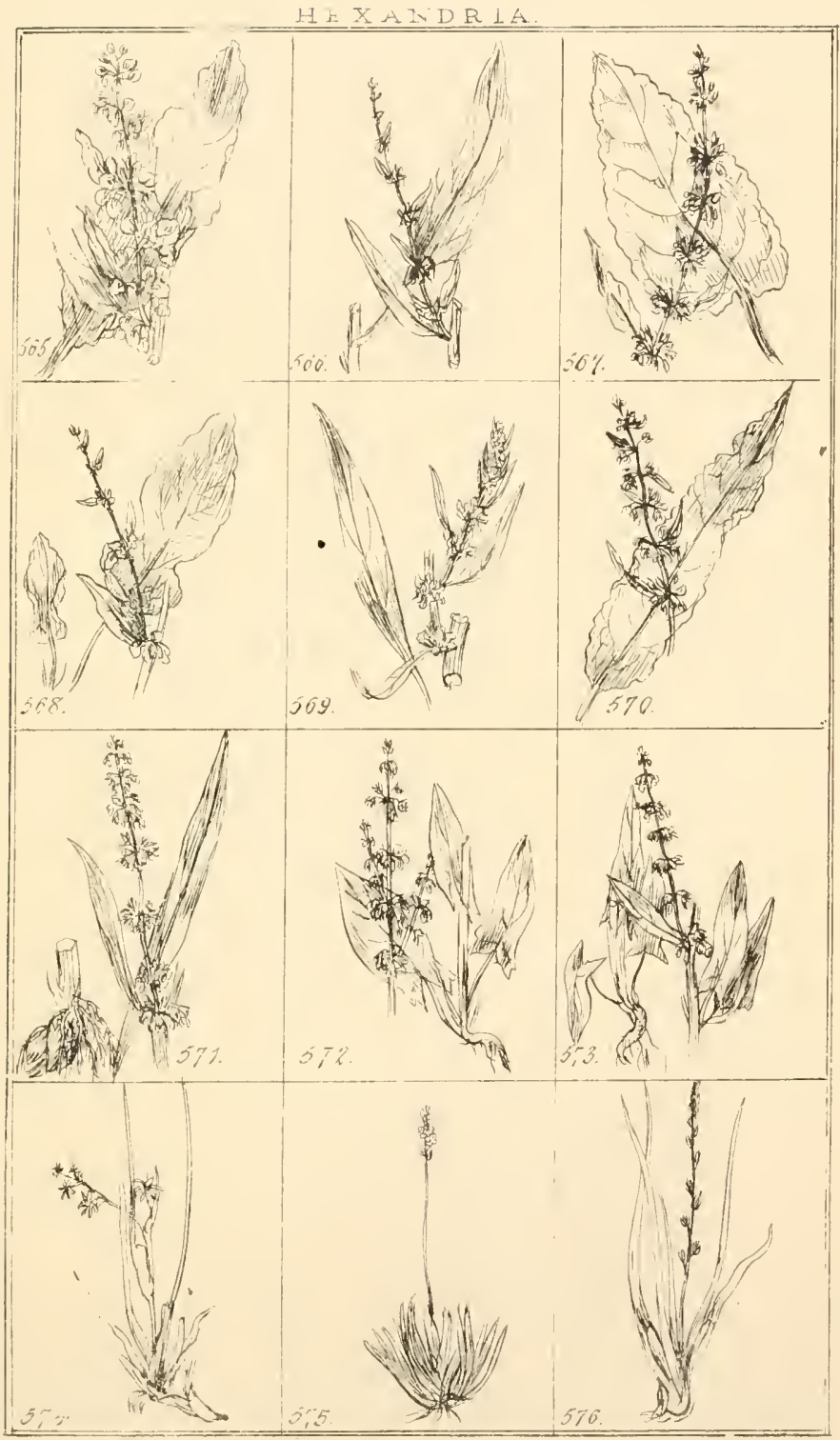





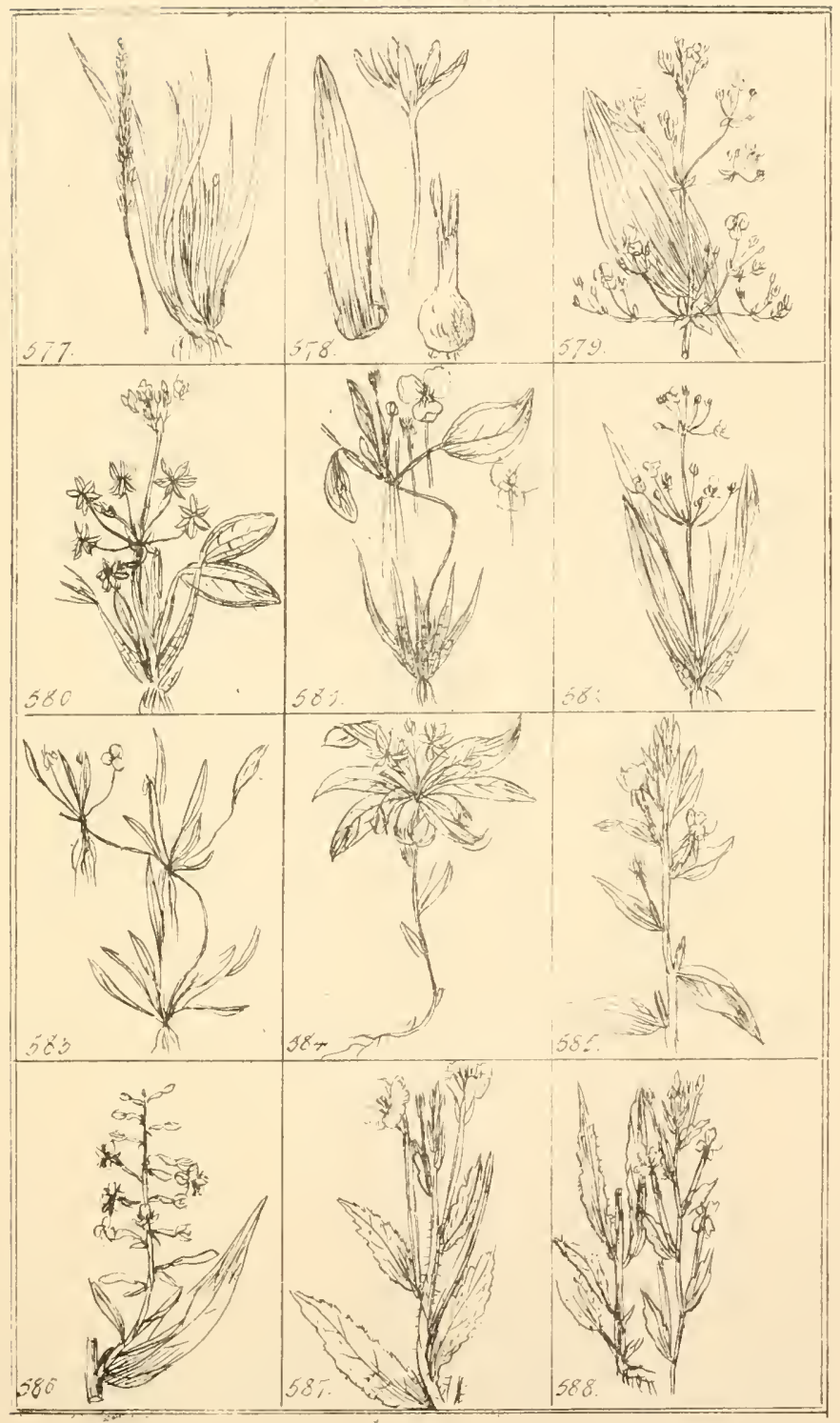





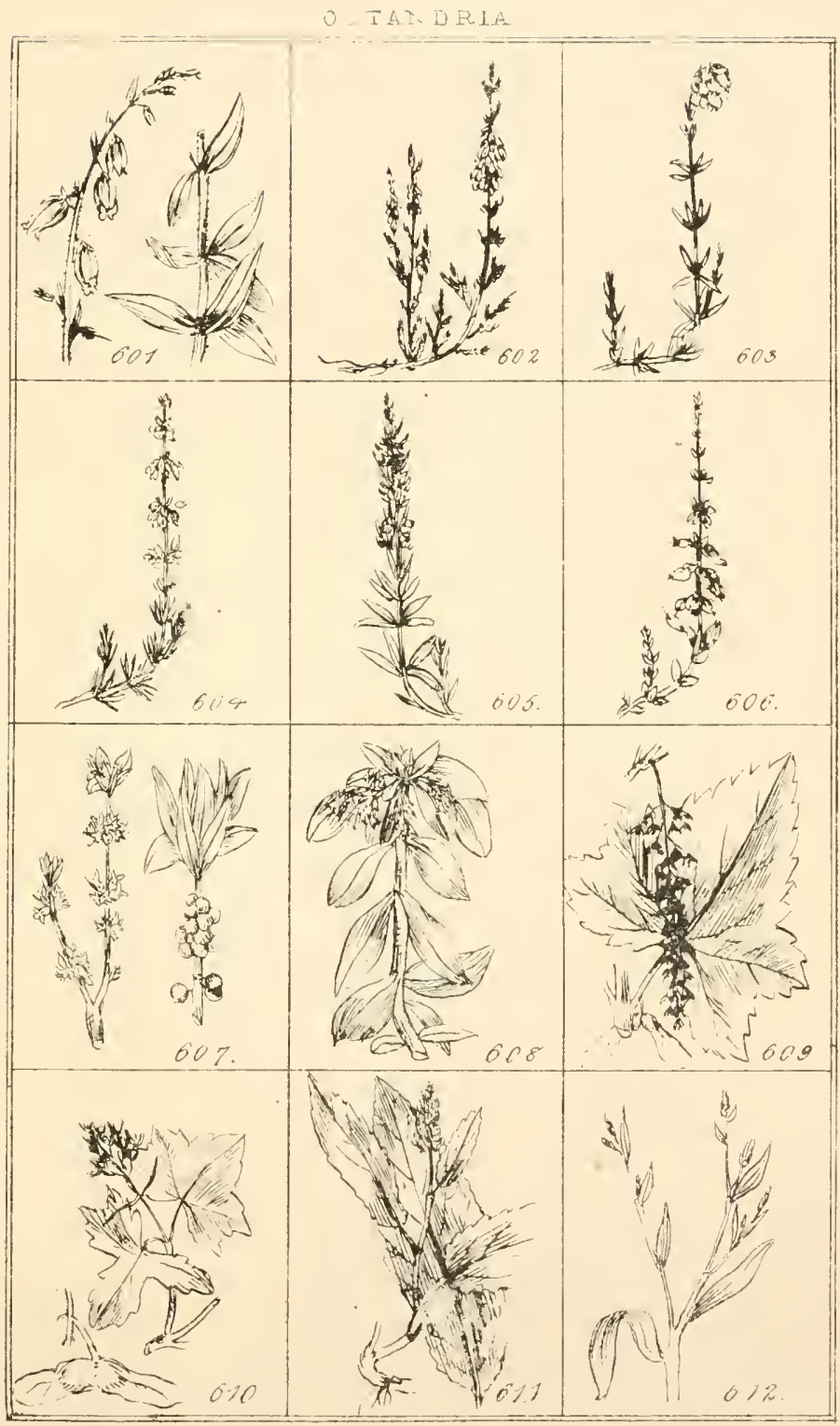





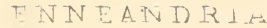

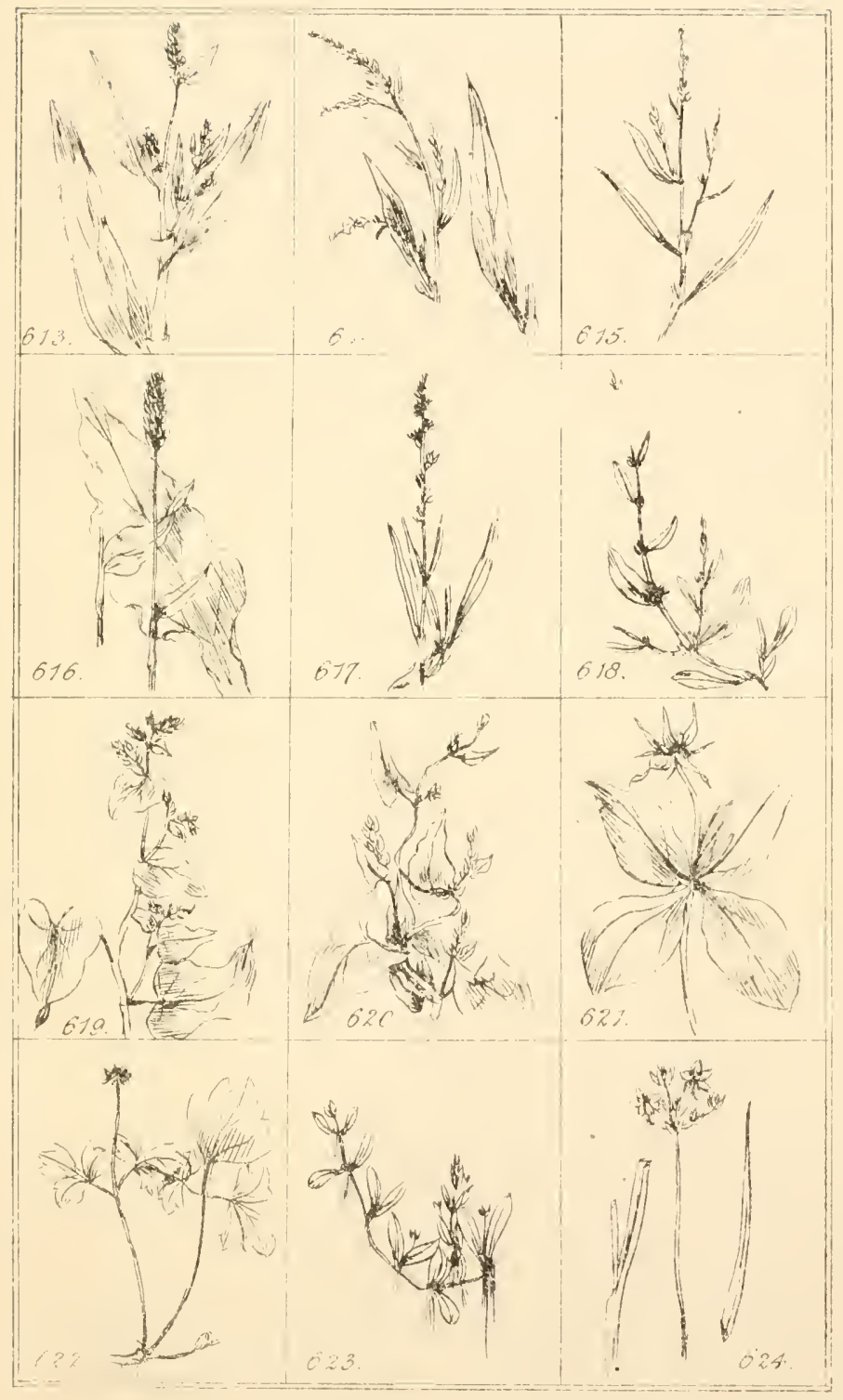





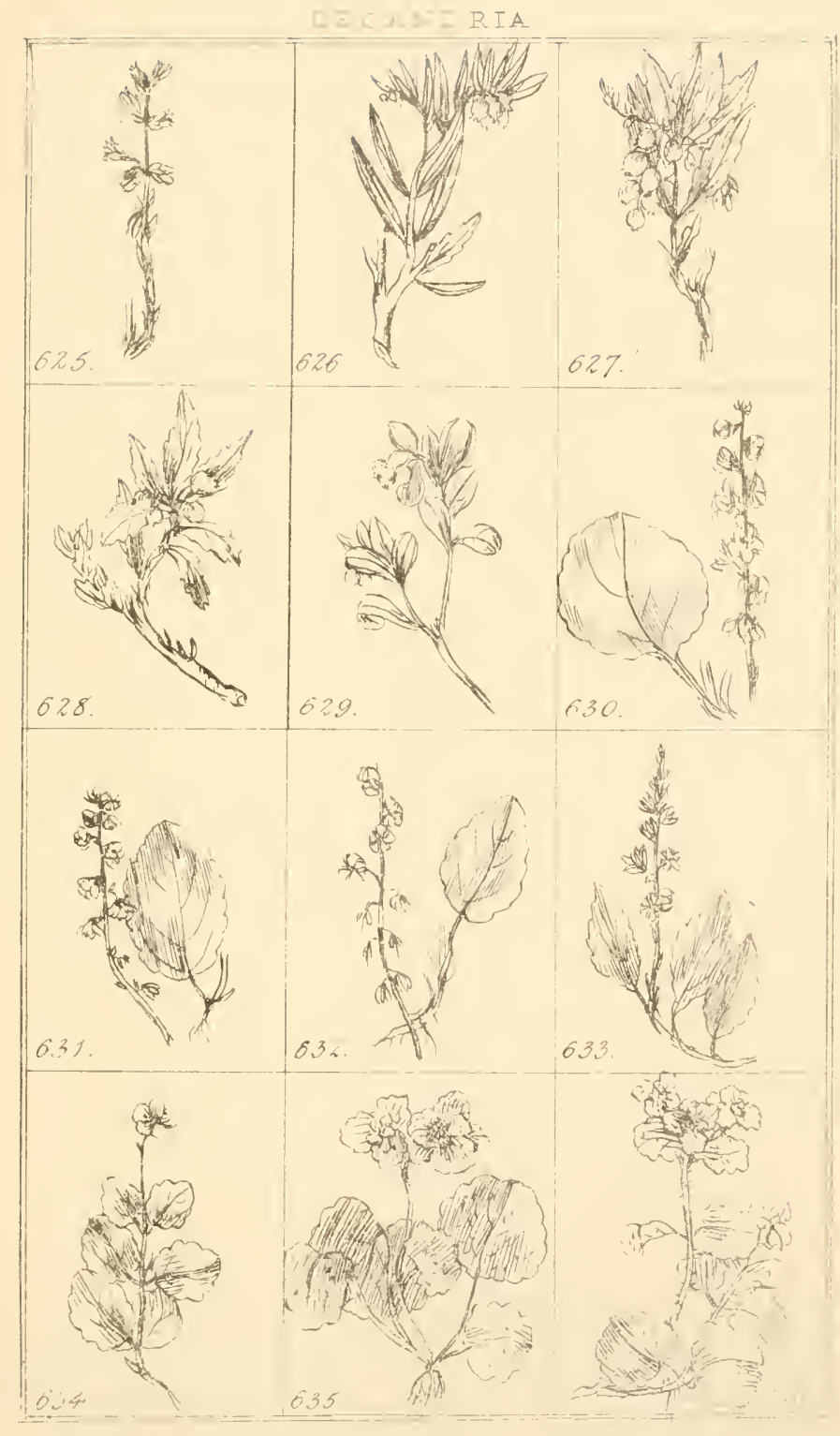





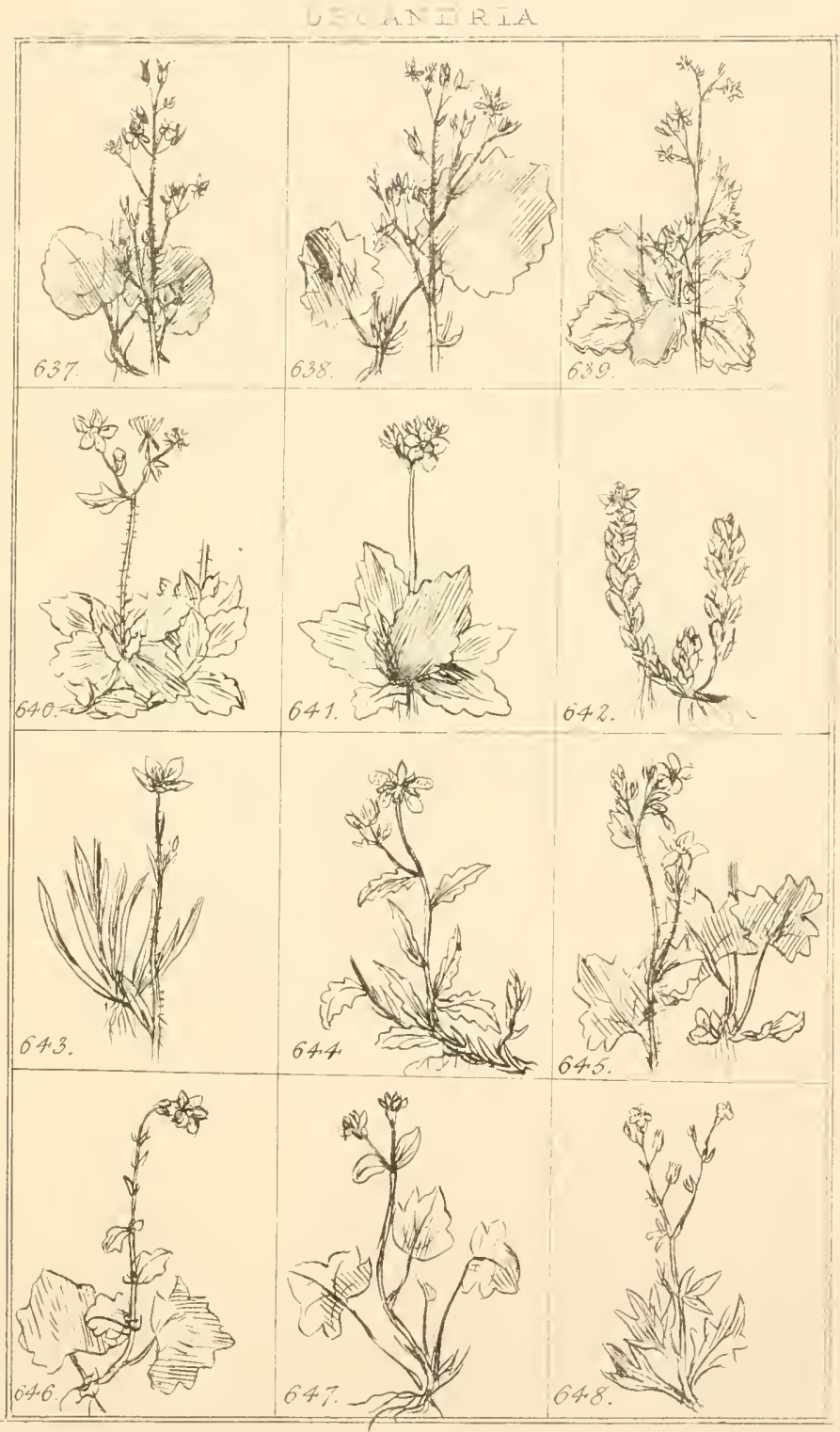



$D E \therefore A$ I $D \perp A$

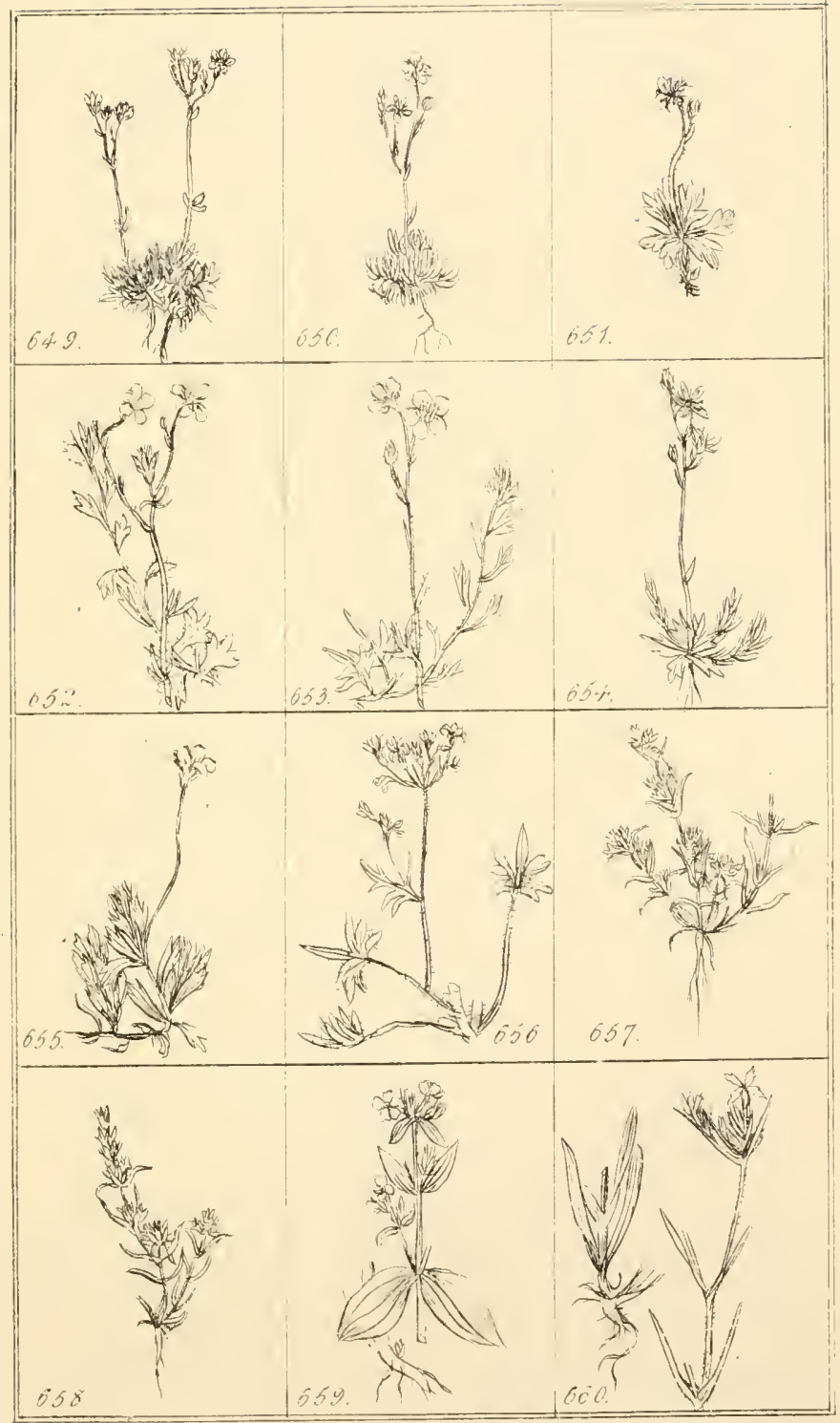





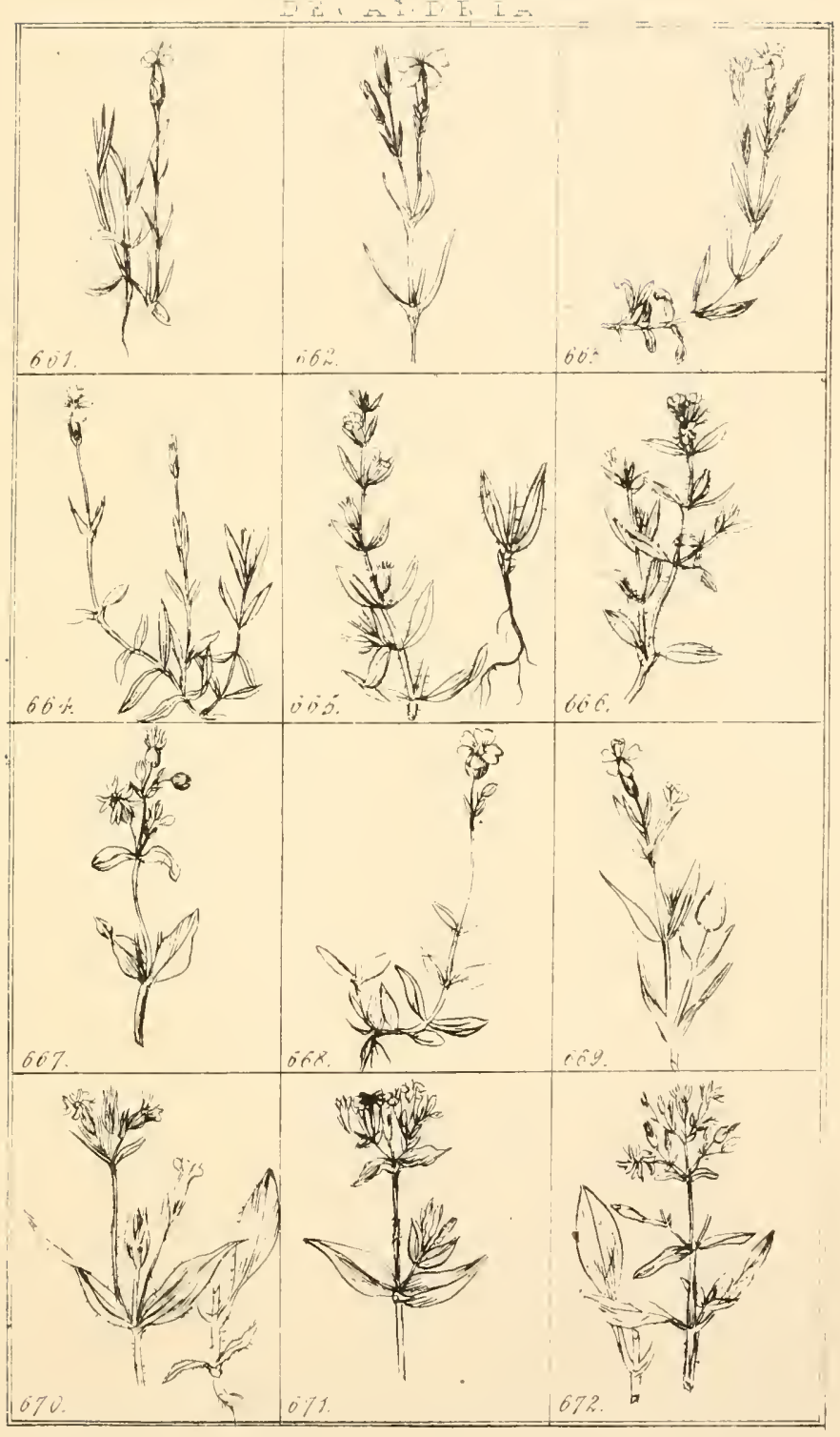





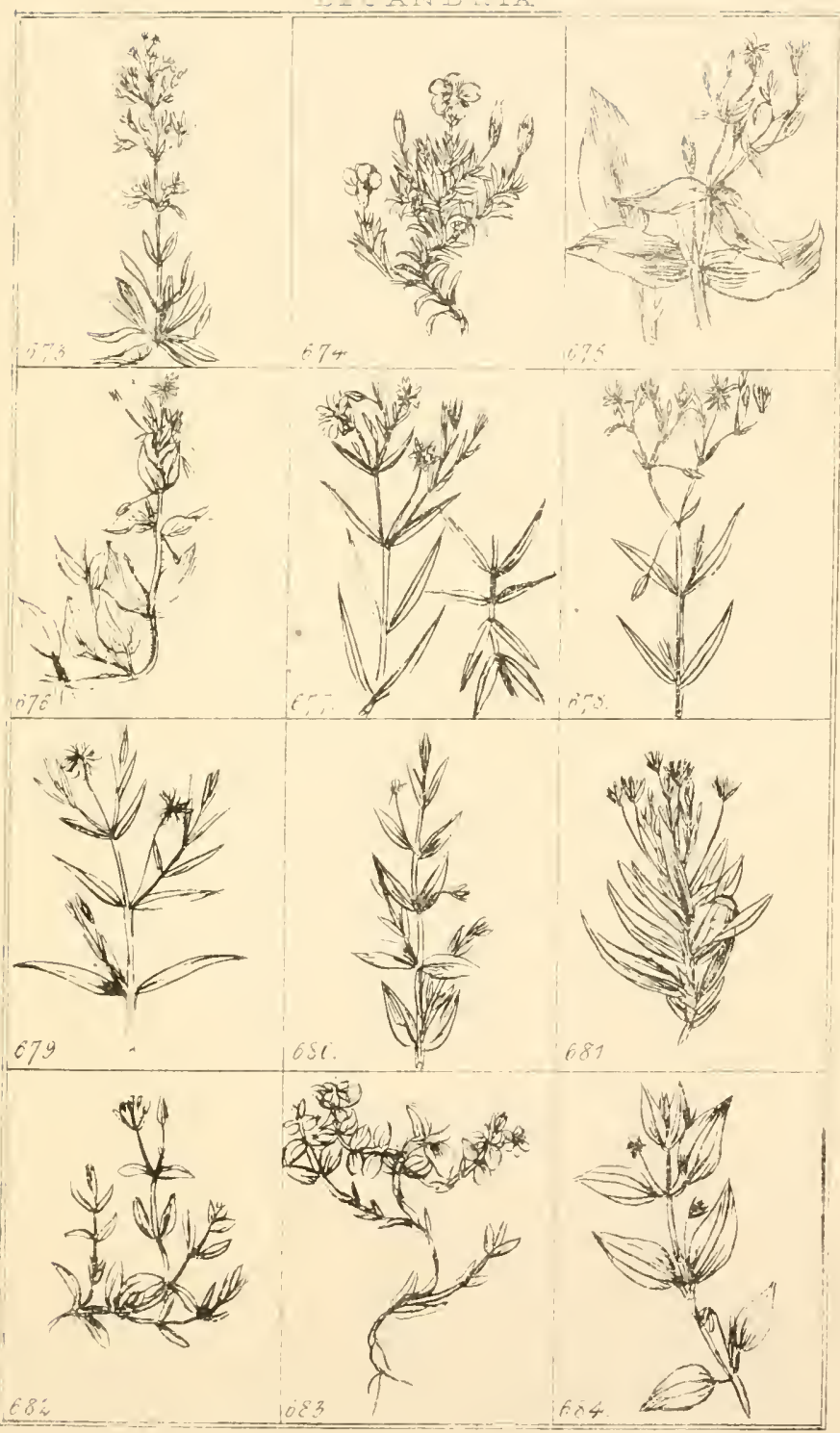





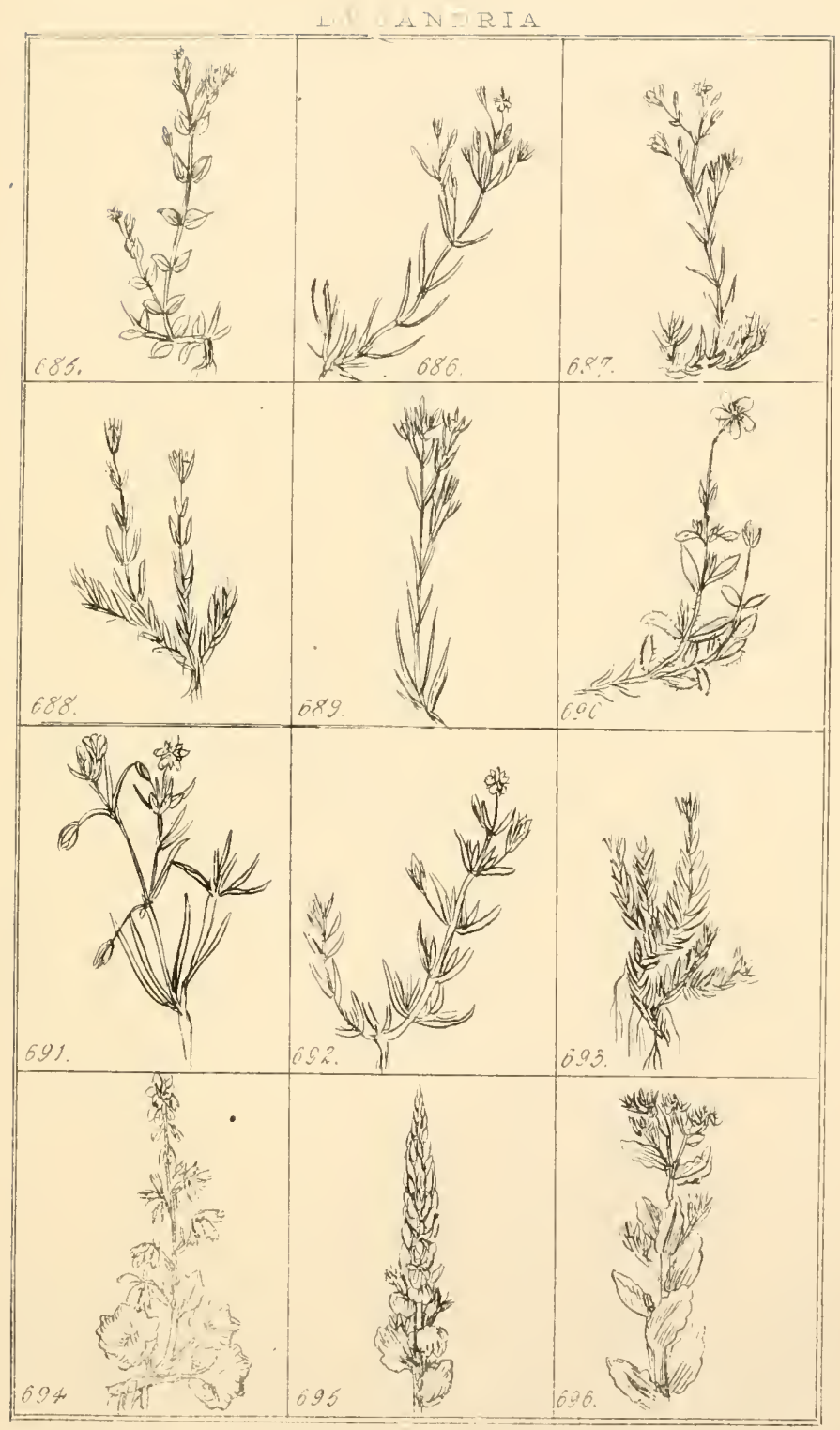



DIDANLRTA

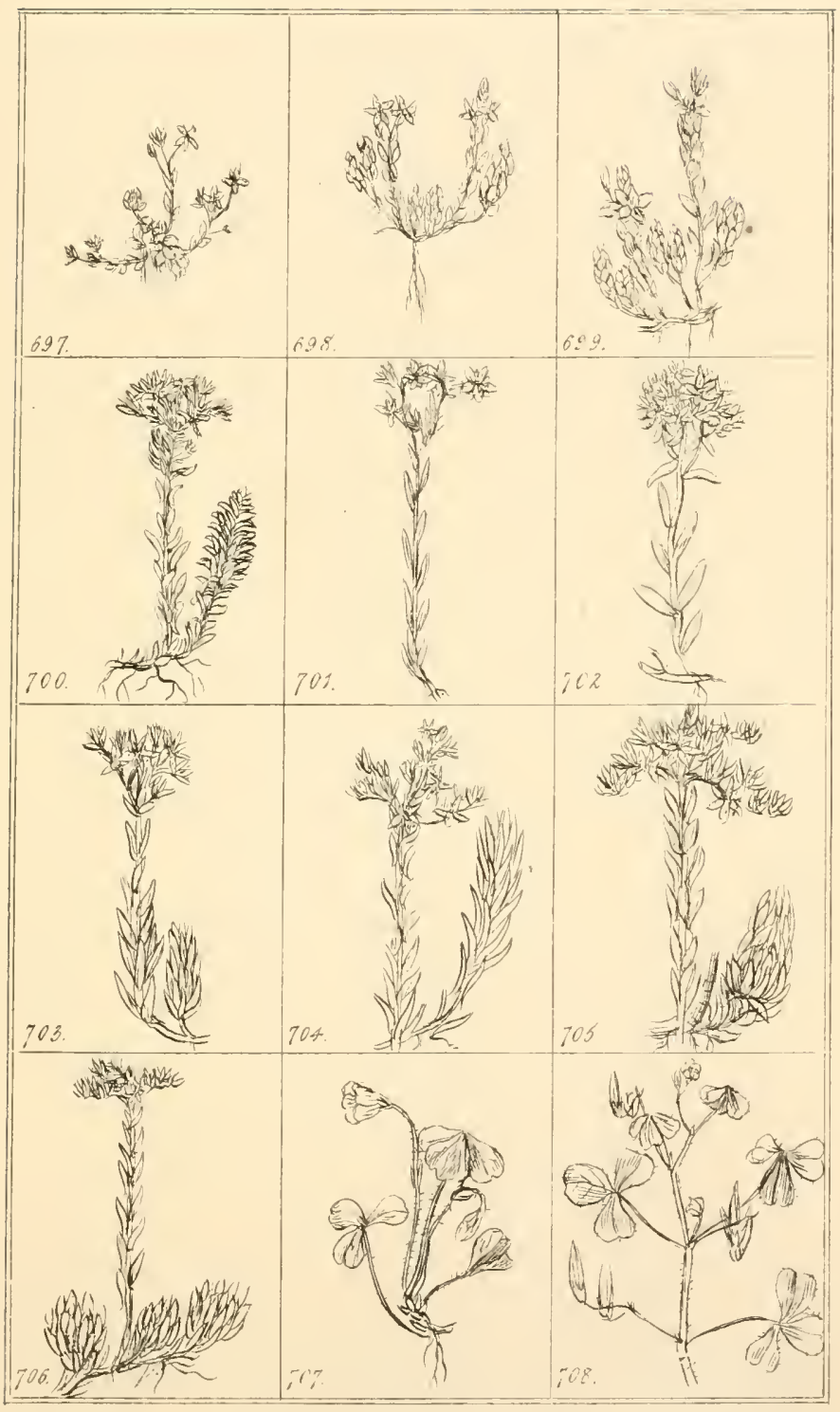



$D \equiv U A N \perp R \perp A$

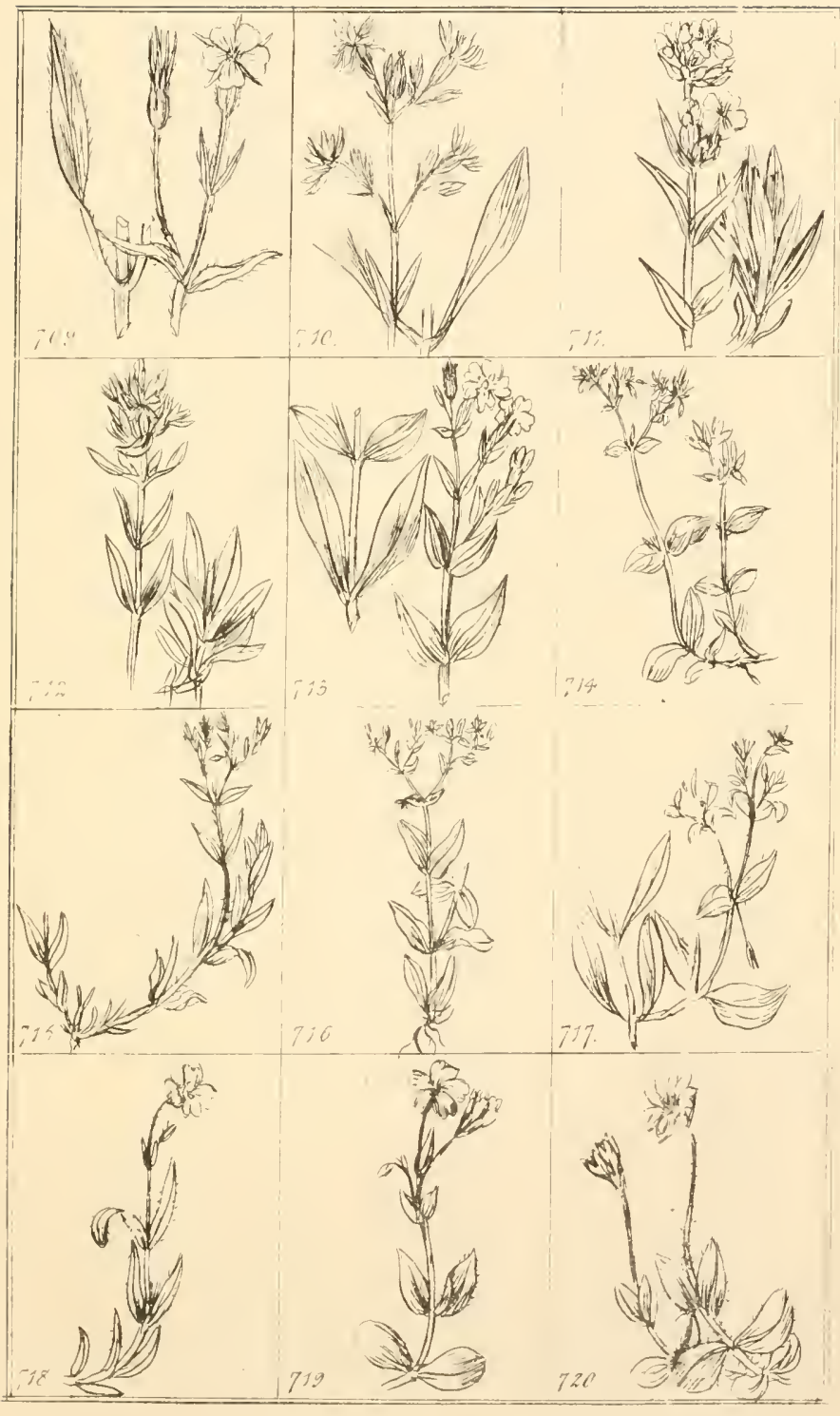





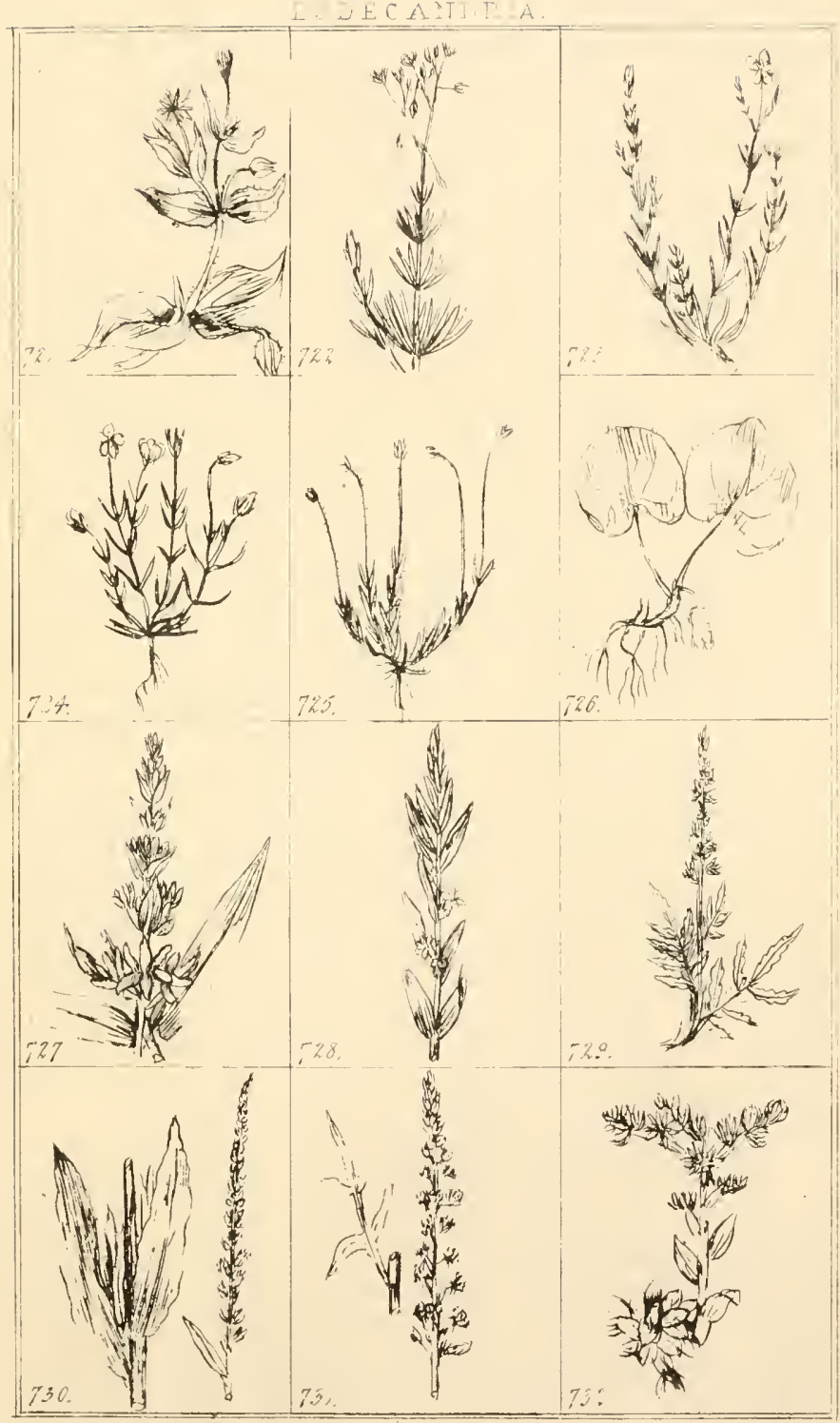





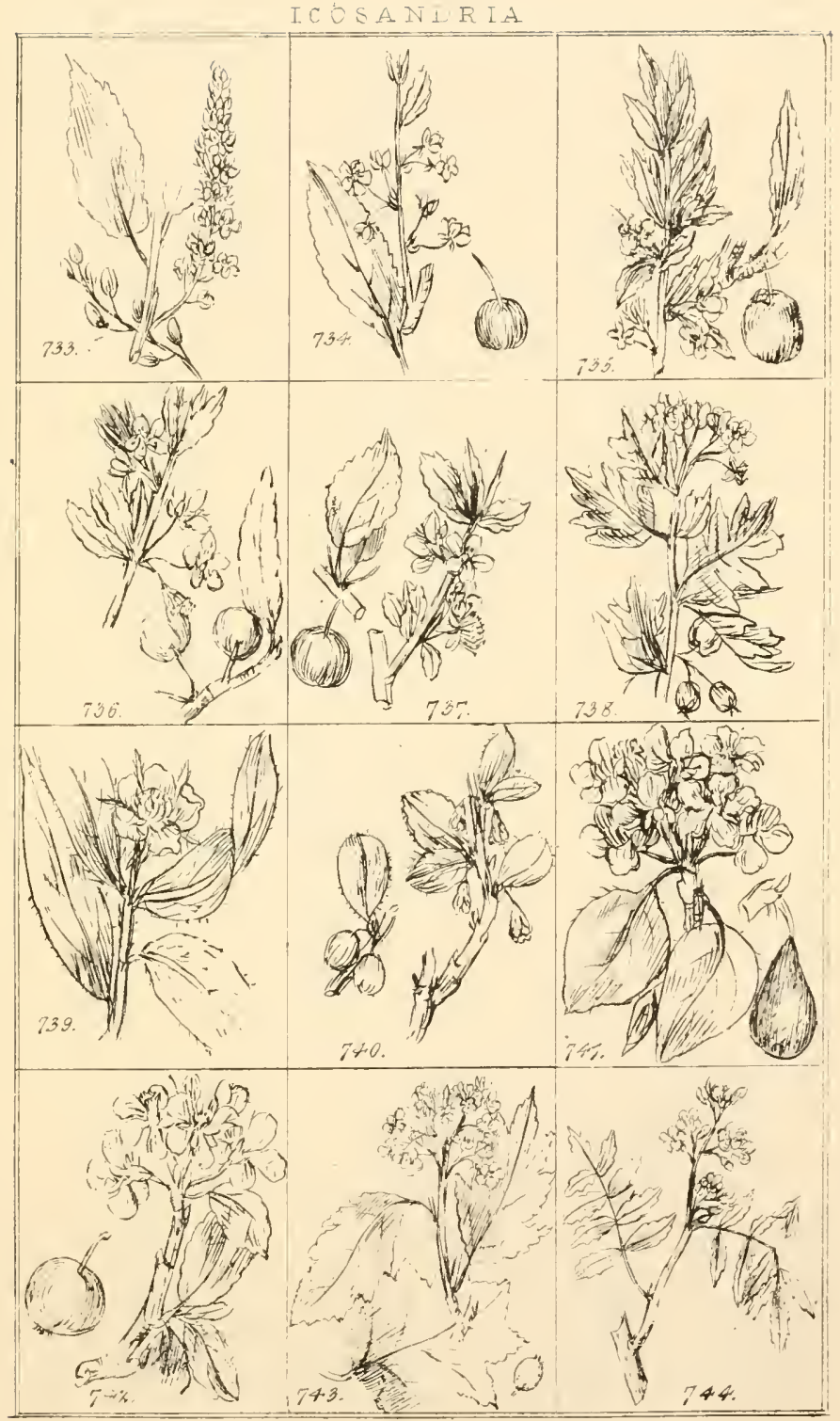



ICOSANDRIA.

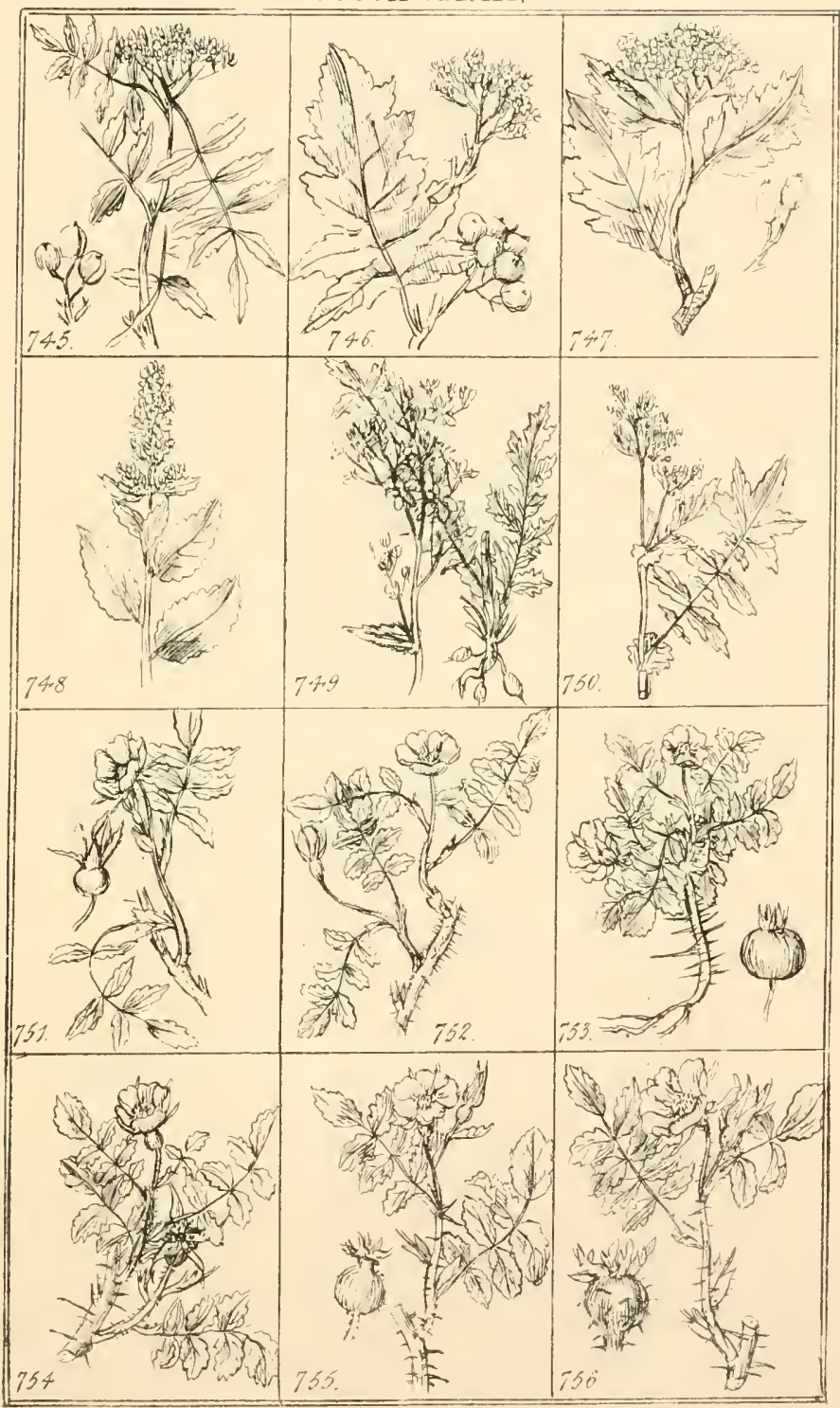



IC SANDRIA.

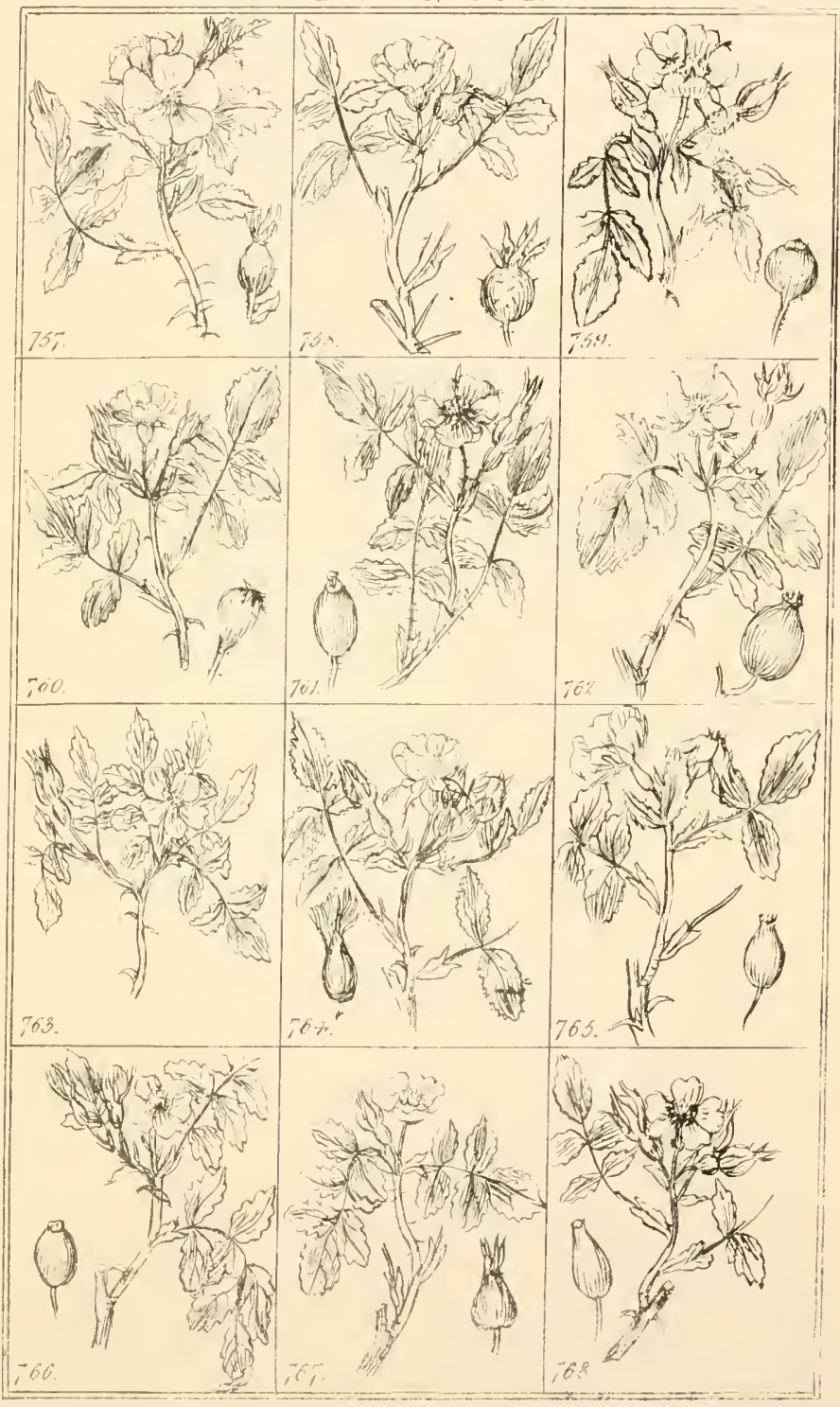





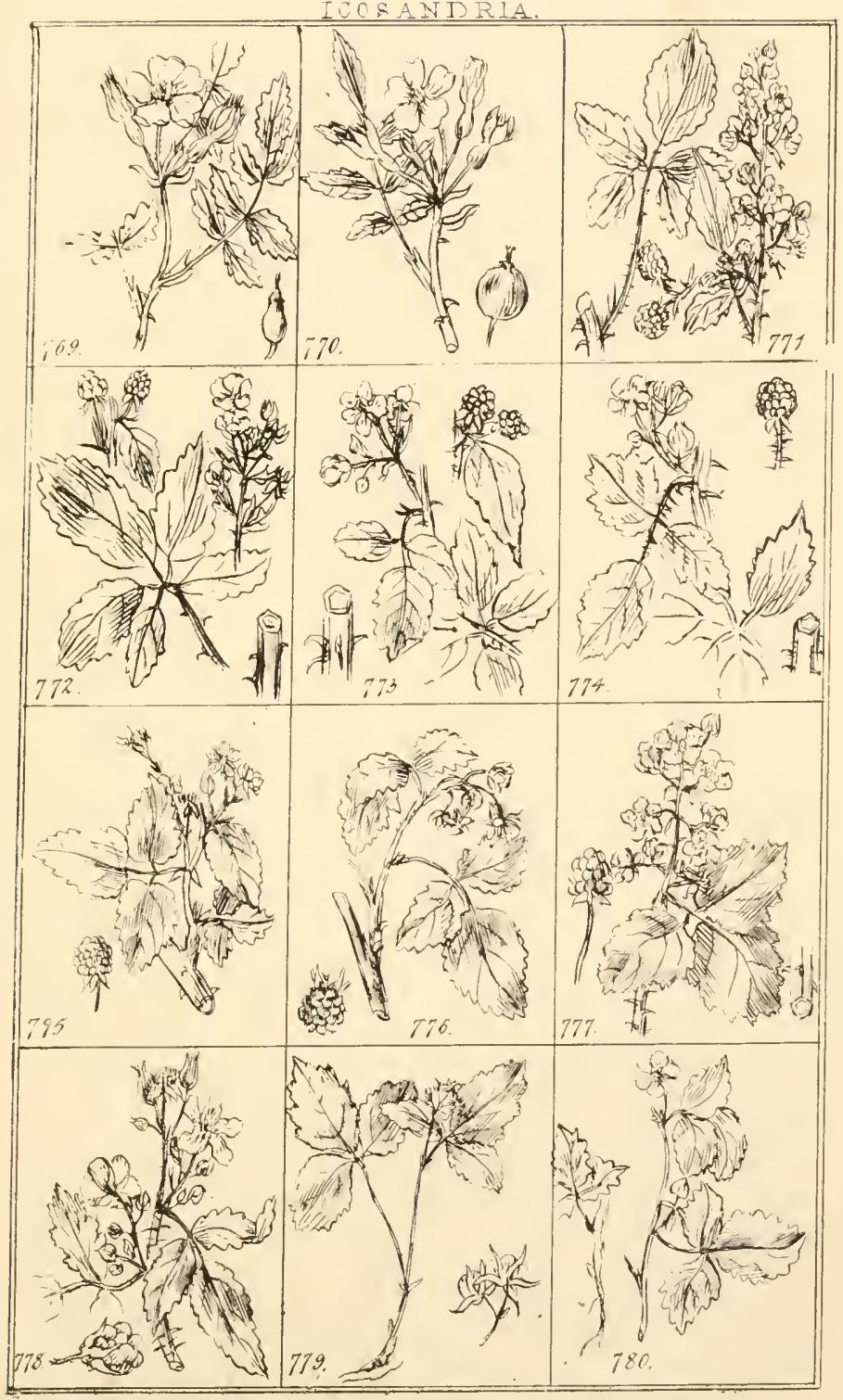



I $O S A N D R I A$

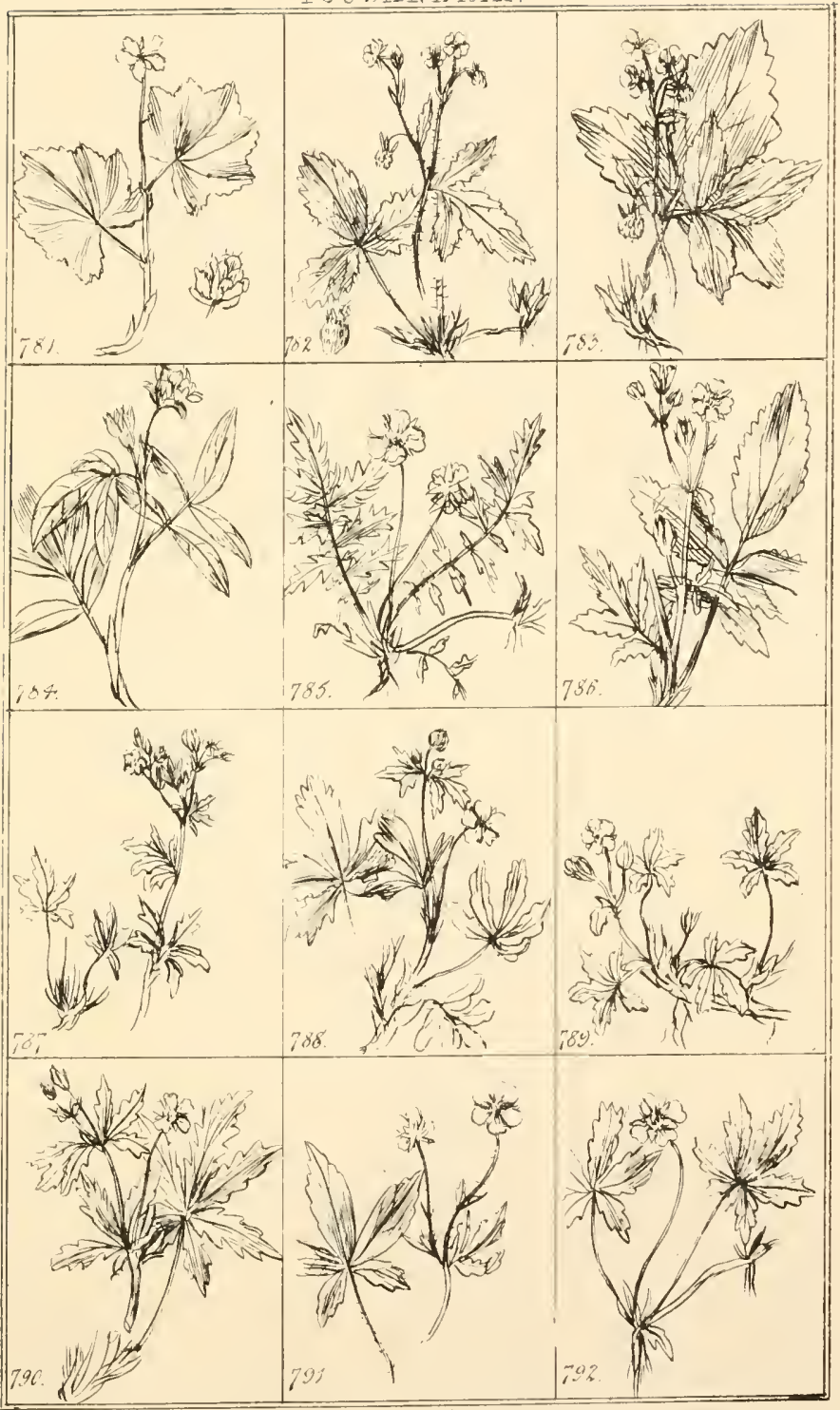





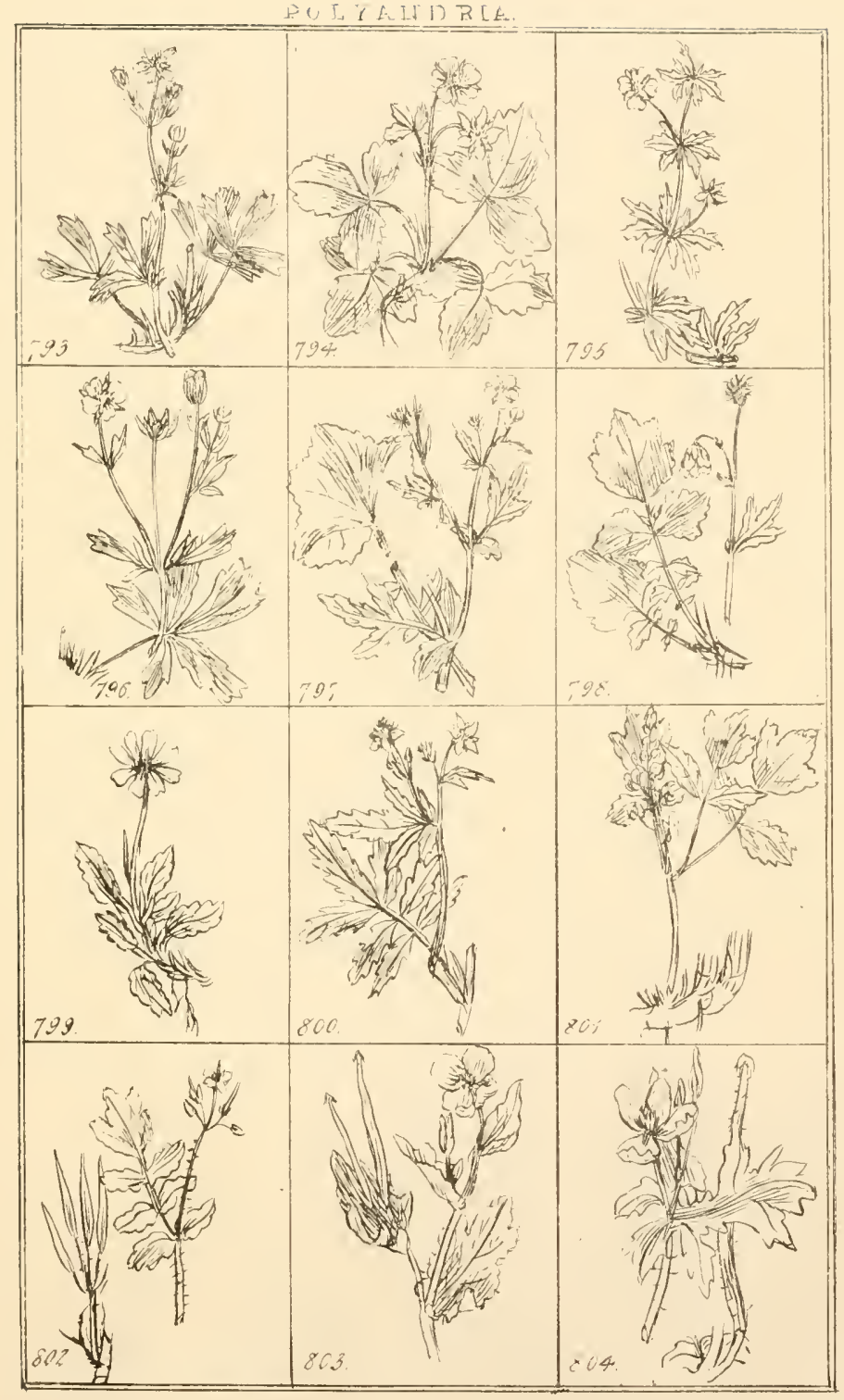



$P \circlearrowleft: Y \in D P R$

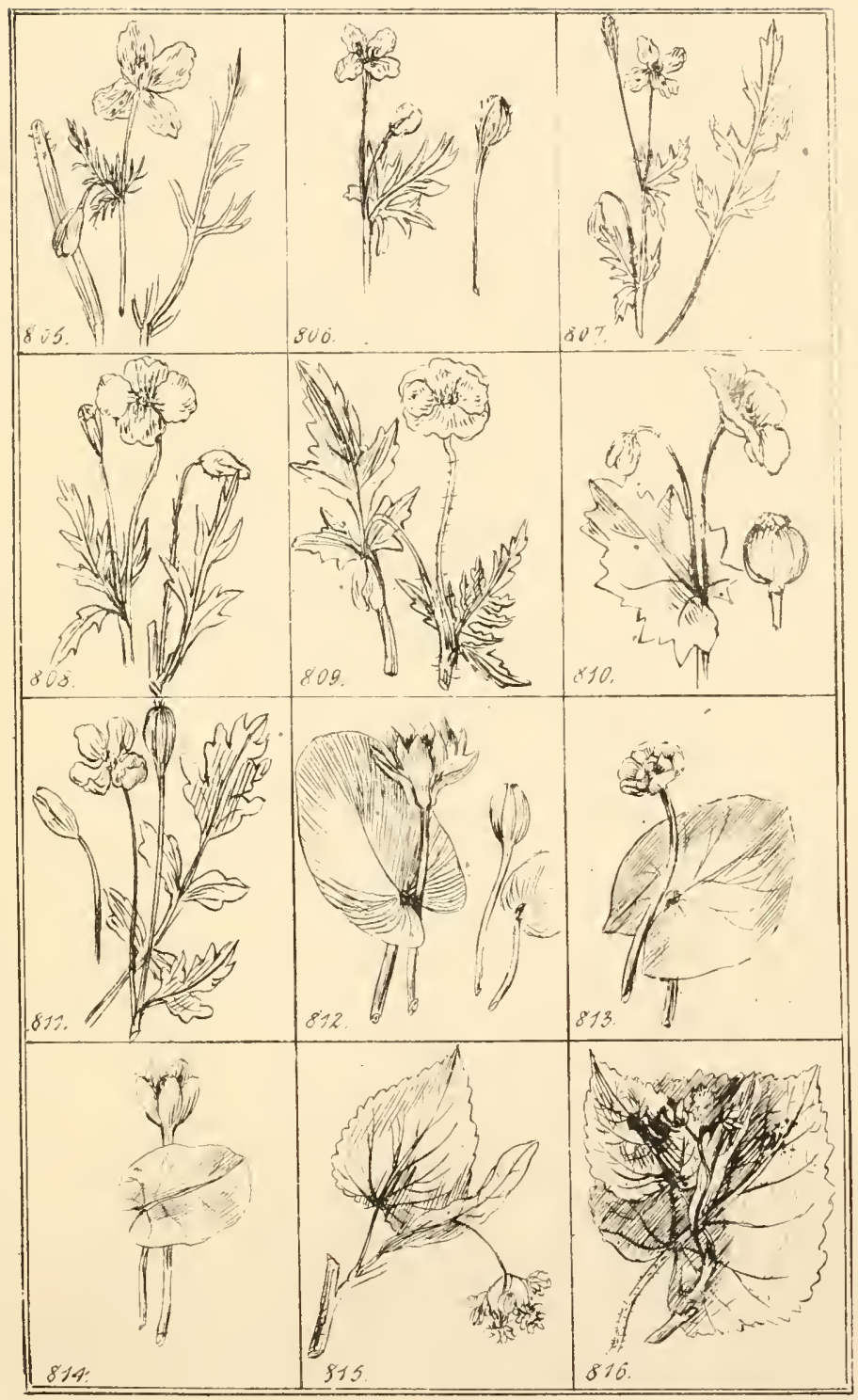





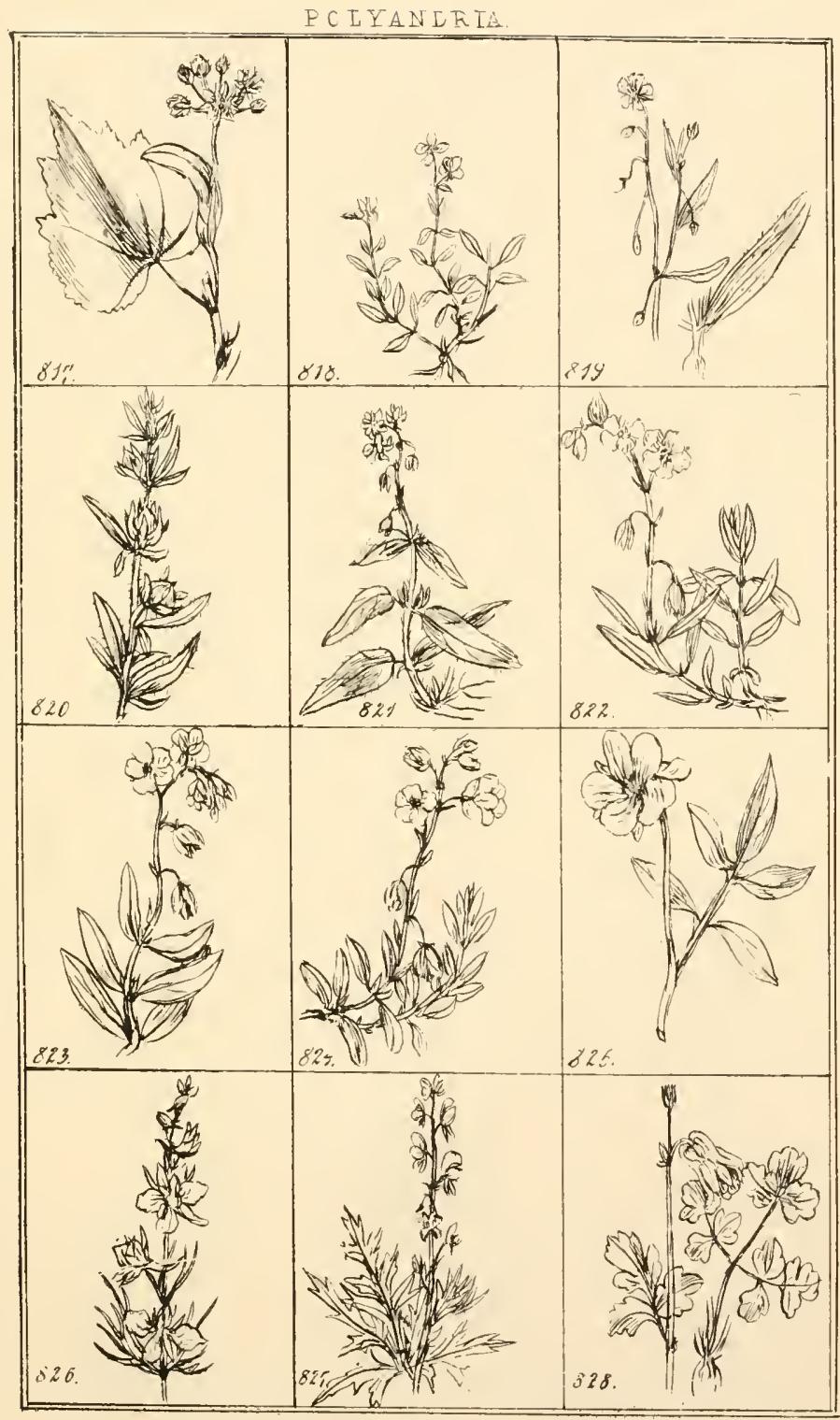





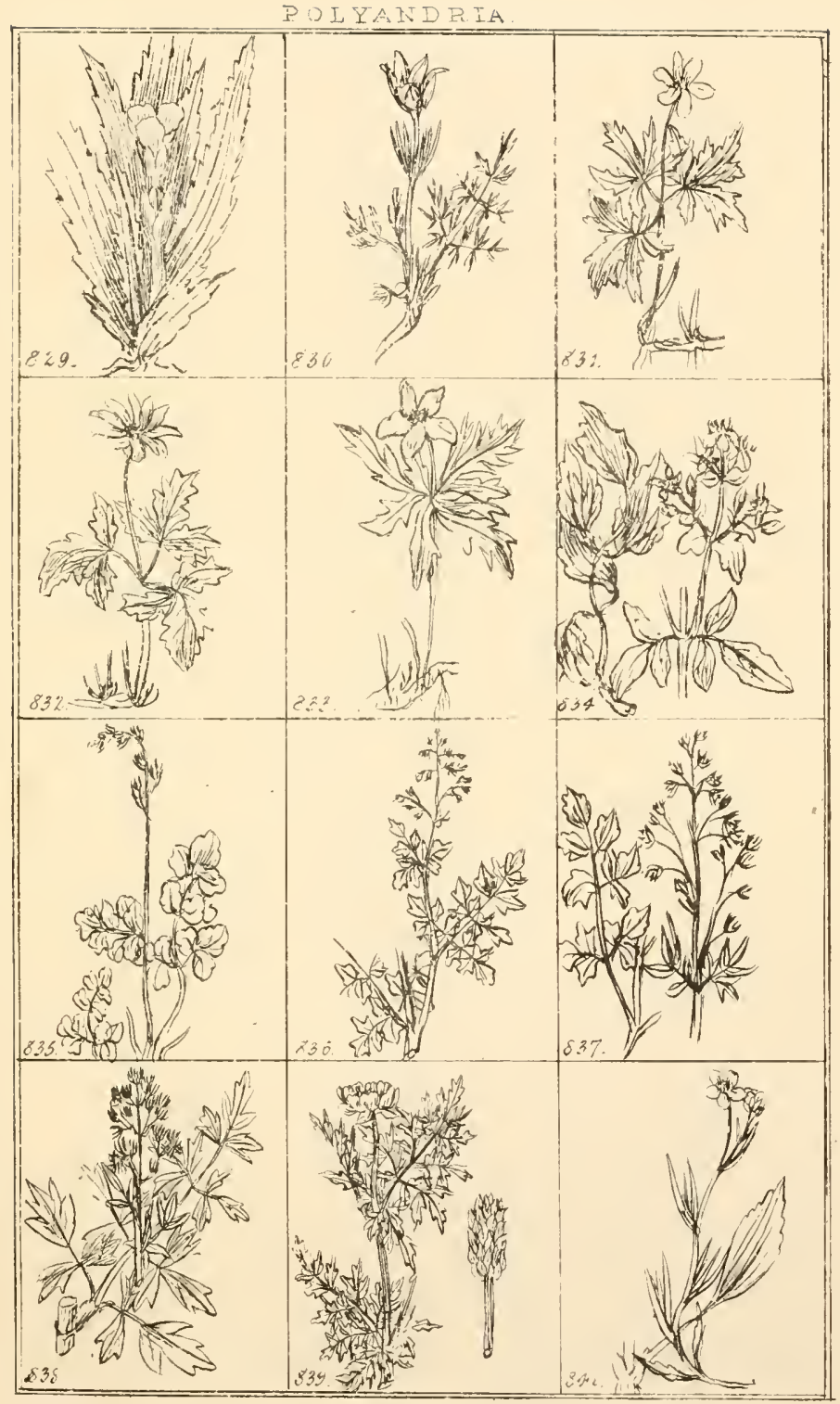



POLYAJDRIA

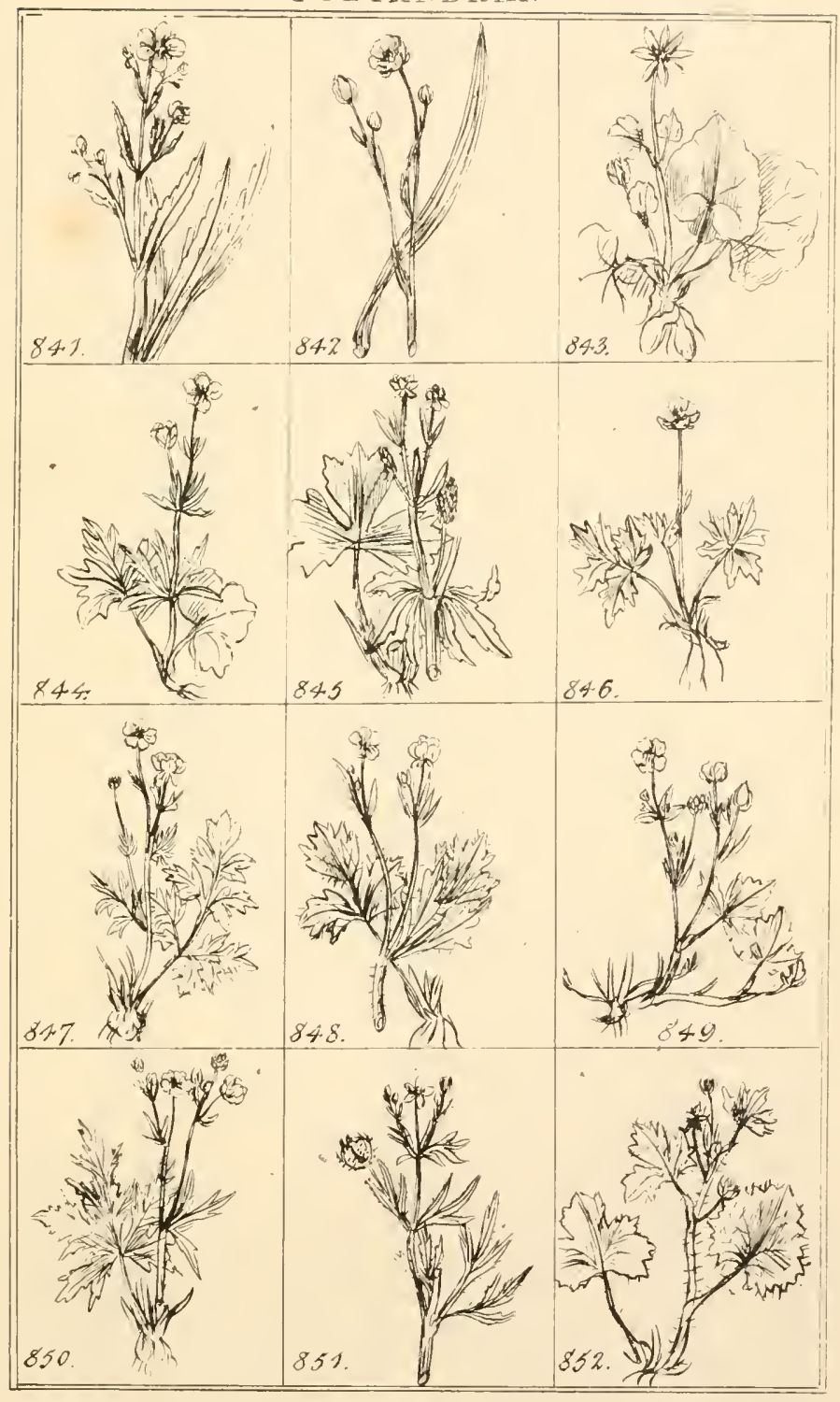



DIDYNAMIA

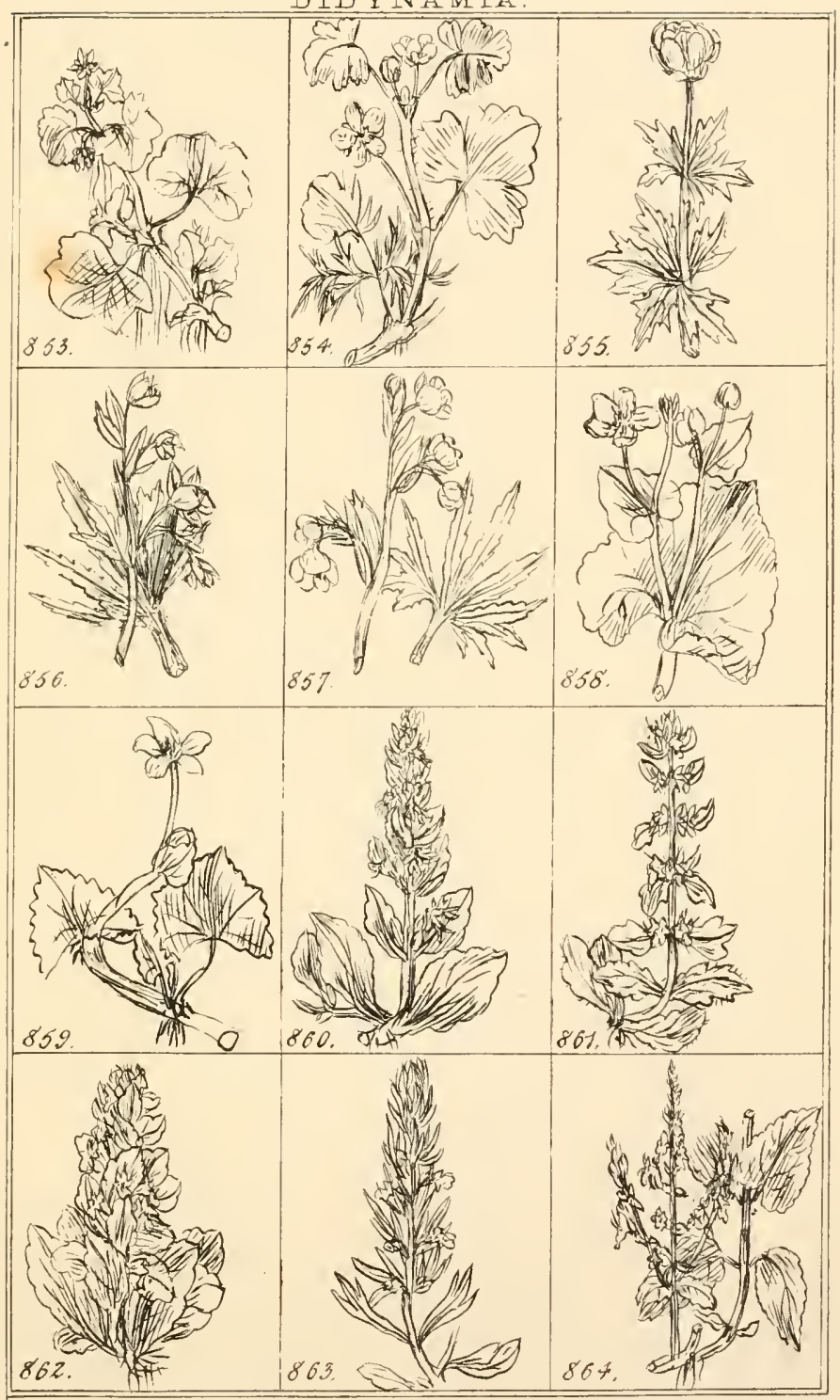



DIDYNAMIA.

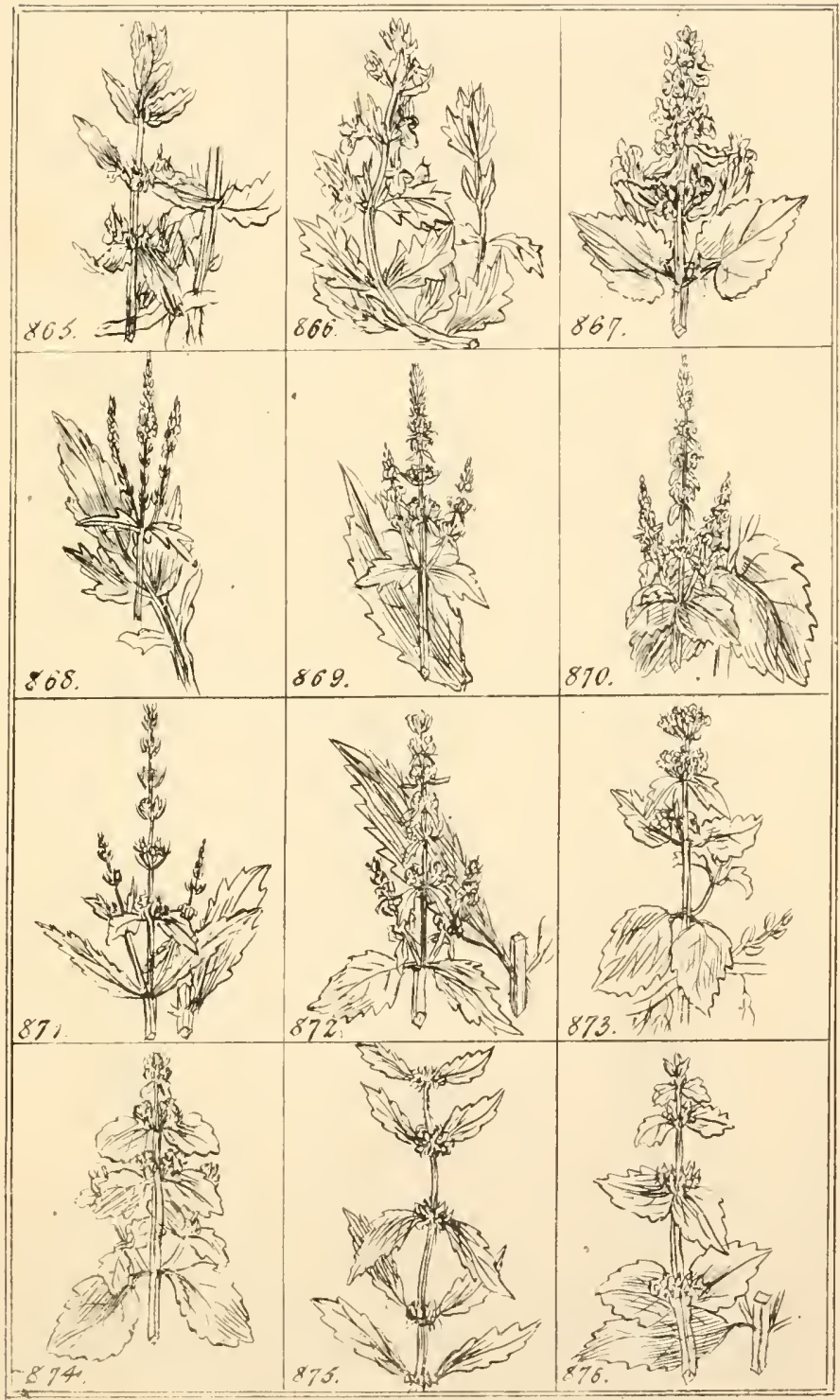



DID IN A MIA.

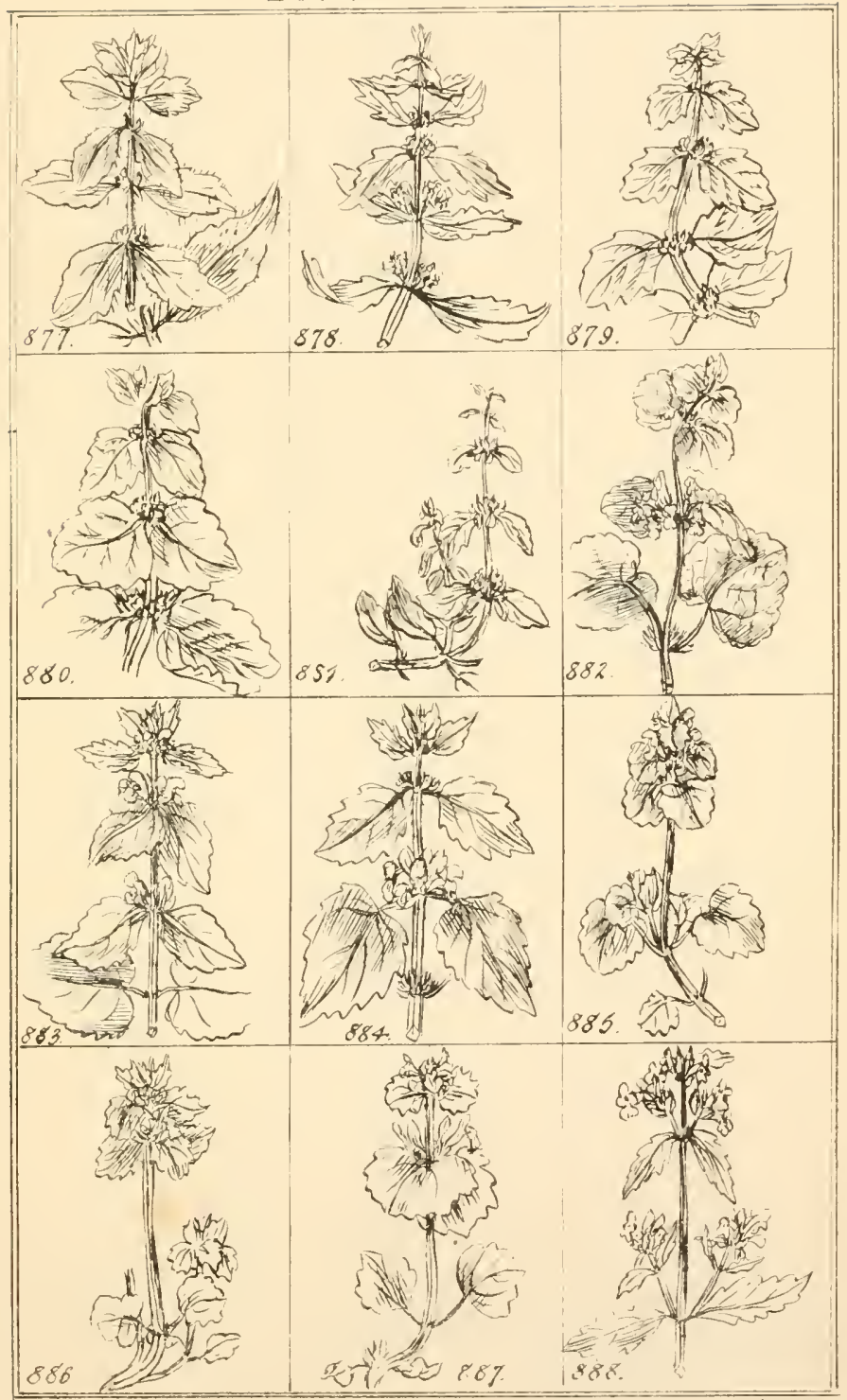



DIDY NAMIA

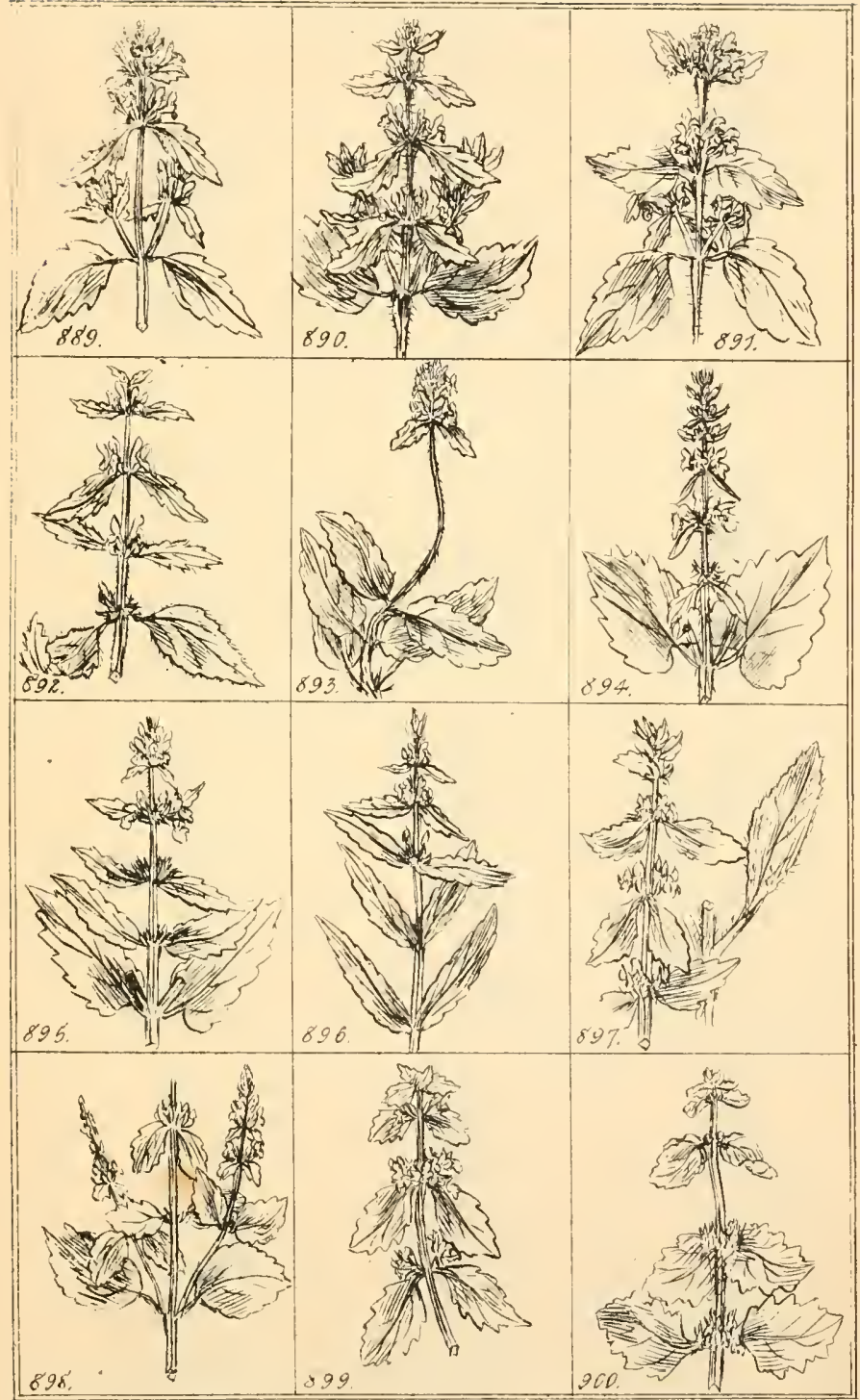



DIDYNAMIA

\begin{tabular}{|c|c|c|}
\hline $\begin{array}{l}4 \\
y \\
y \\
y\end{array}$ & $\begin{array}{c}4 \\
0 \\
0\end{array}$ & (1) \\
\hline 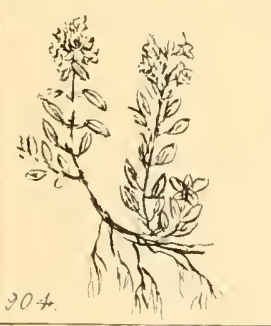 & 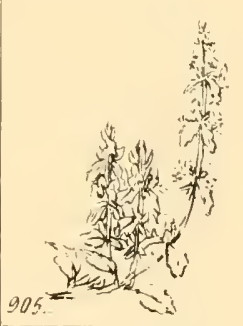 & 称 \\
\hline 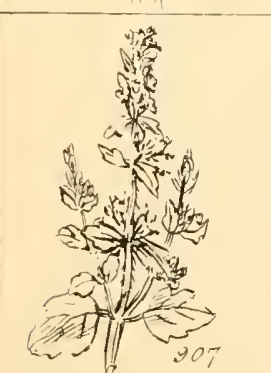 & sill & 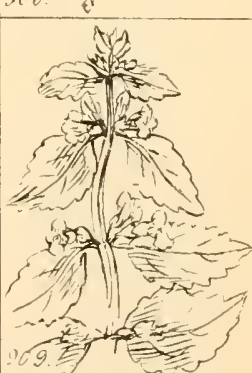 \\
\hline 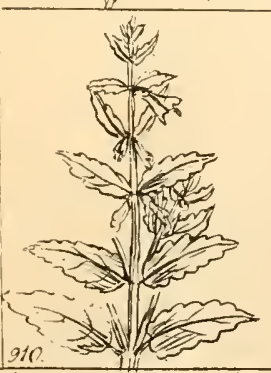 & (1) & at \\
\hline
\end{tabular}



$D i E, Y L, M j A$

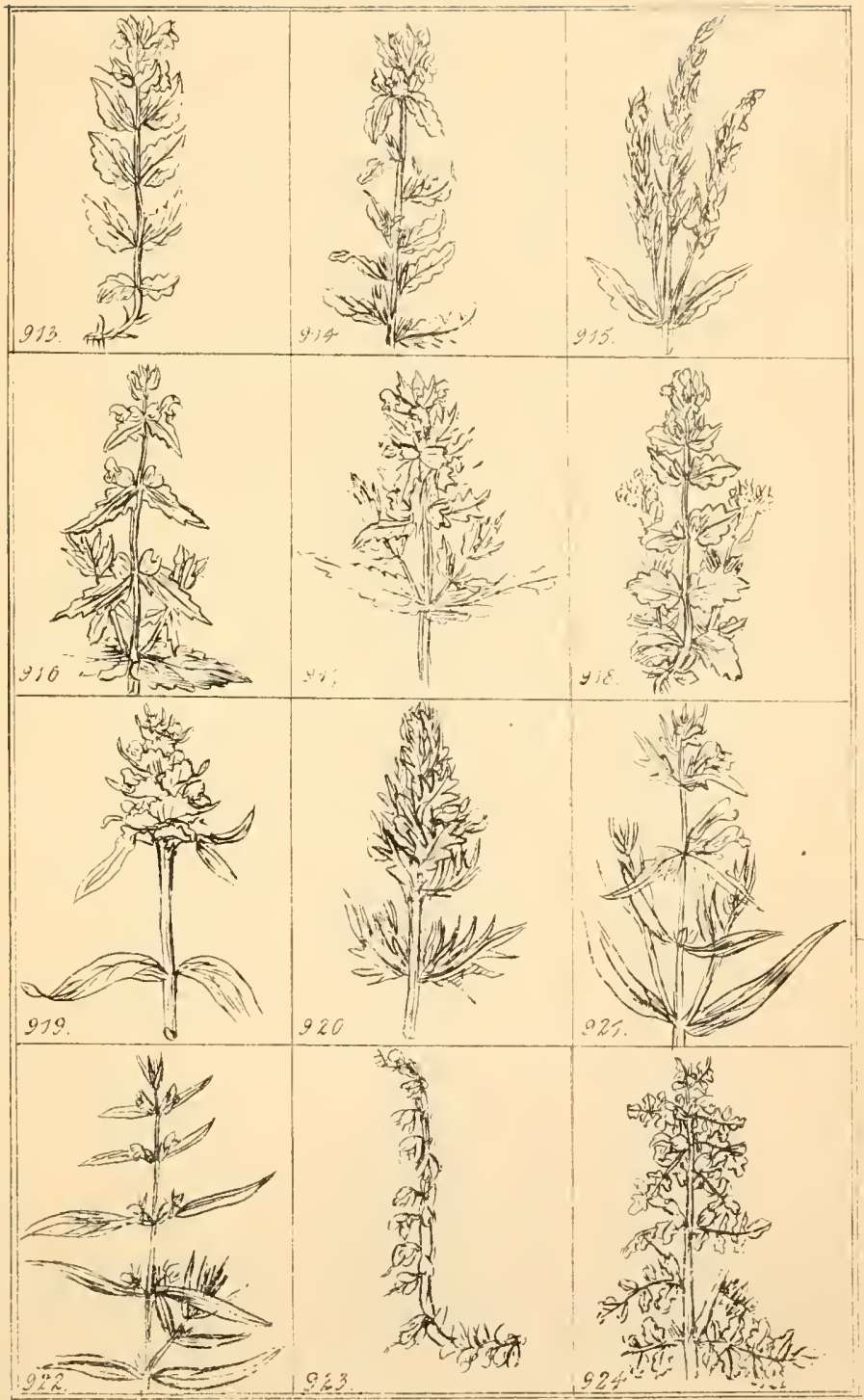



DII INA MIA

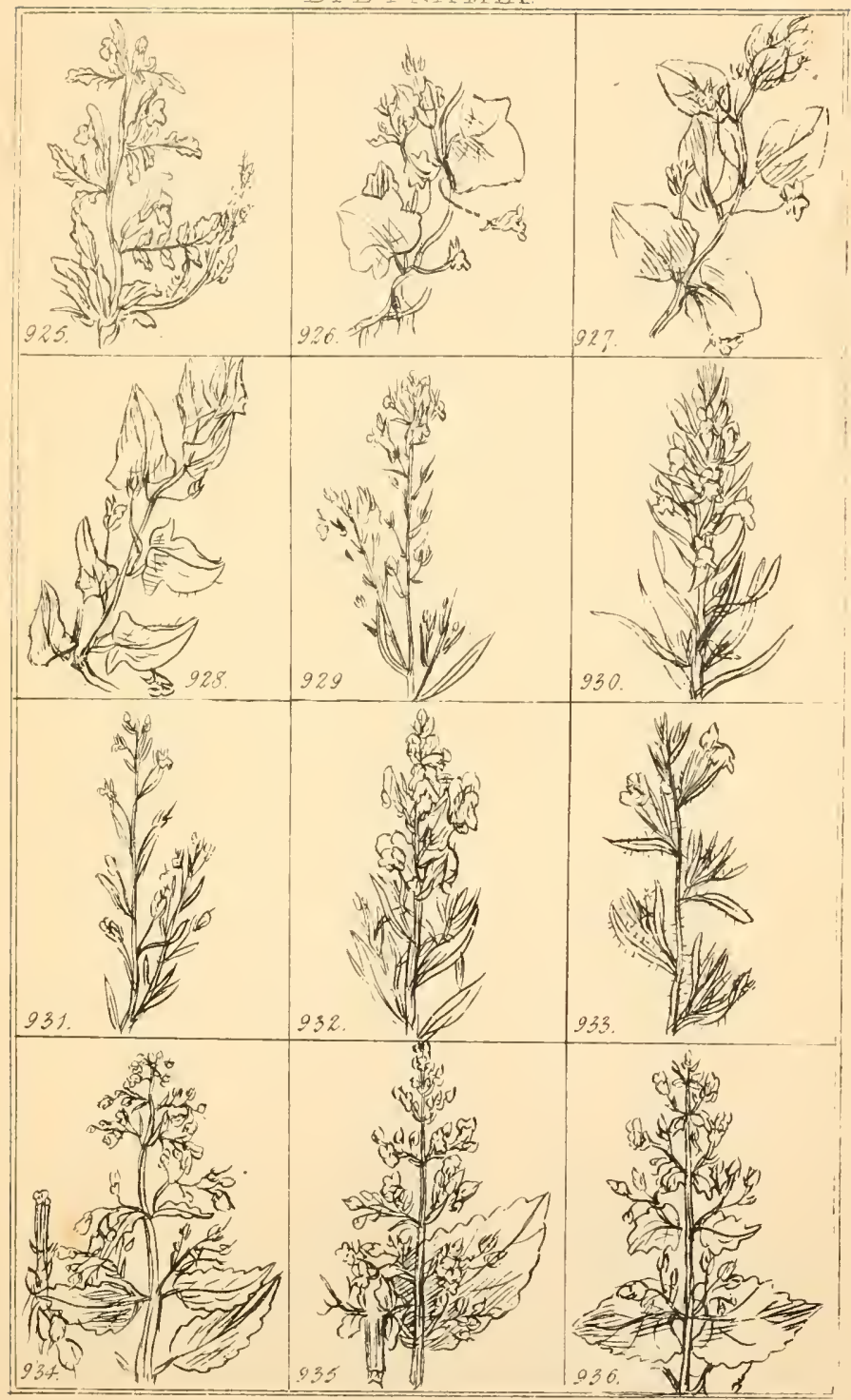





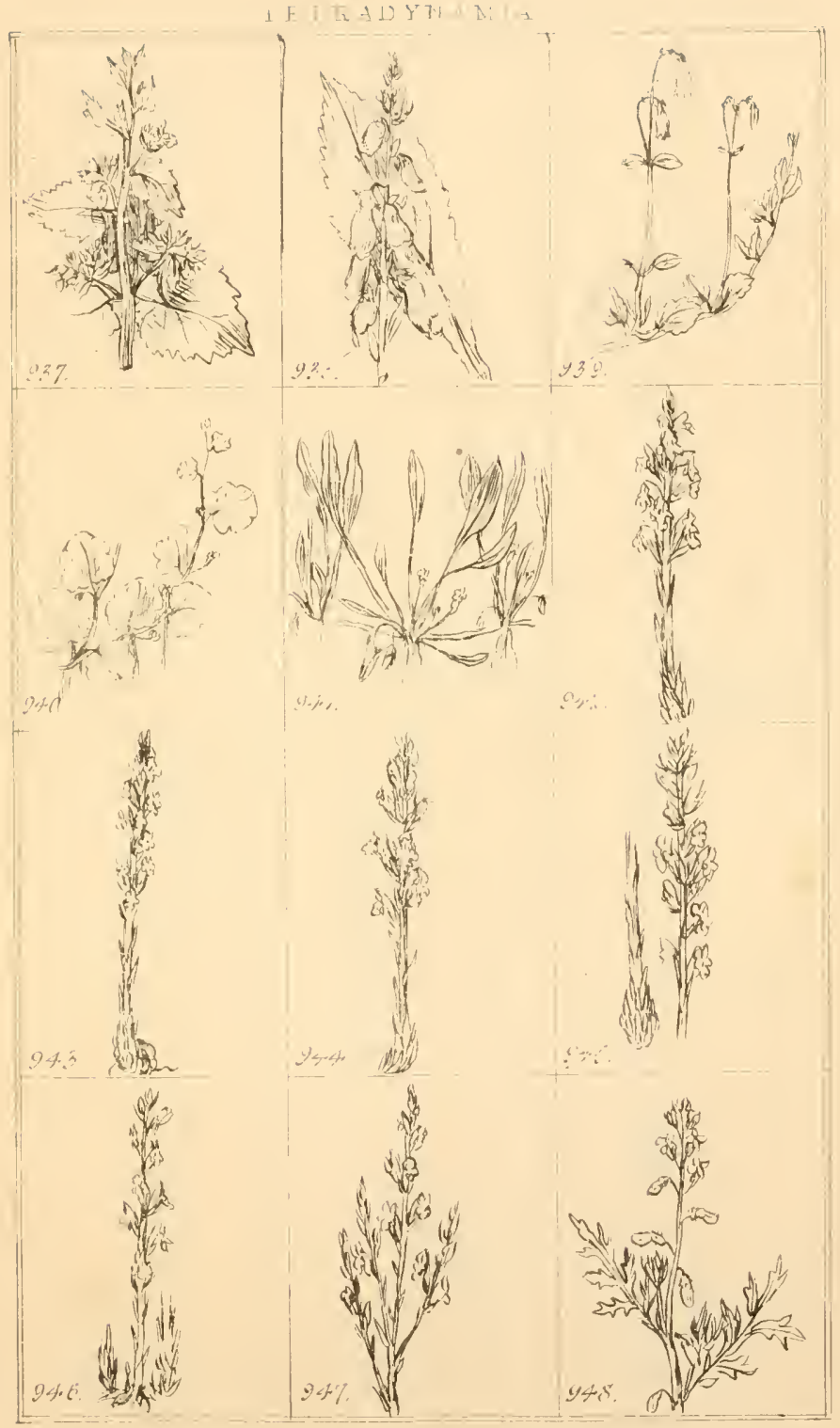





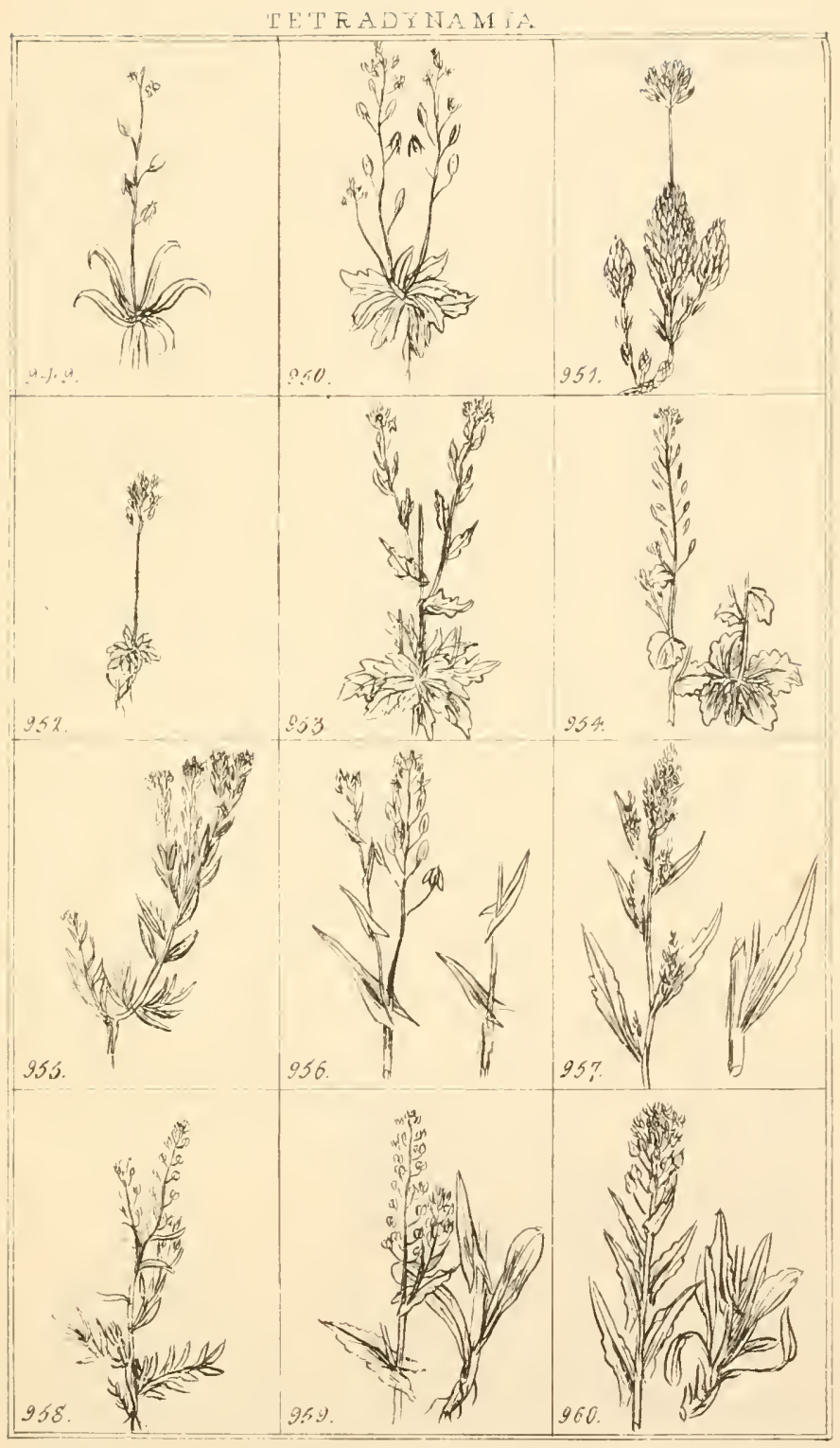



TETRADYITAMIA.

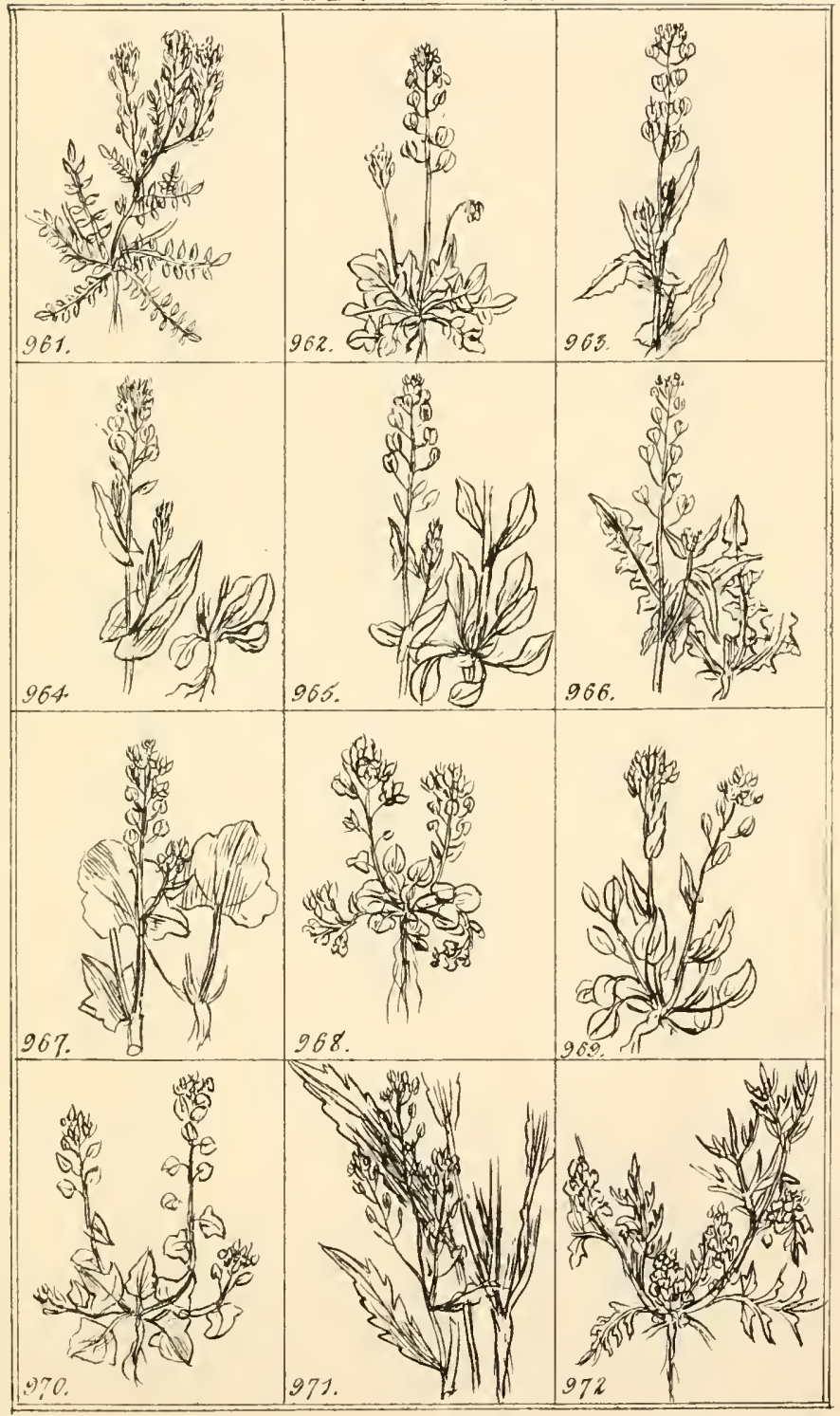



TEI P ADYNA

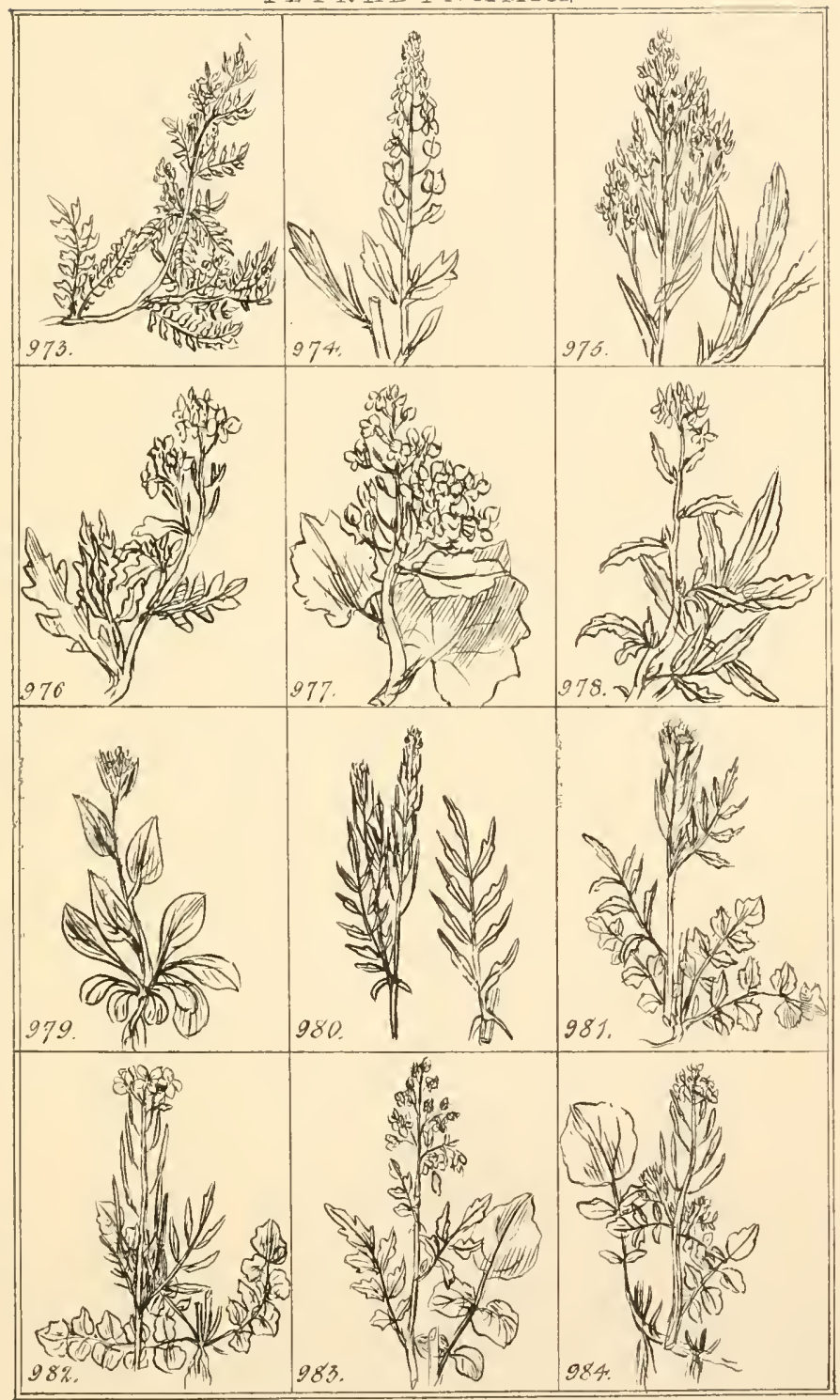



TETEADYNAMIA.

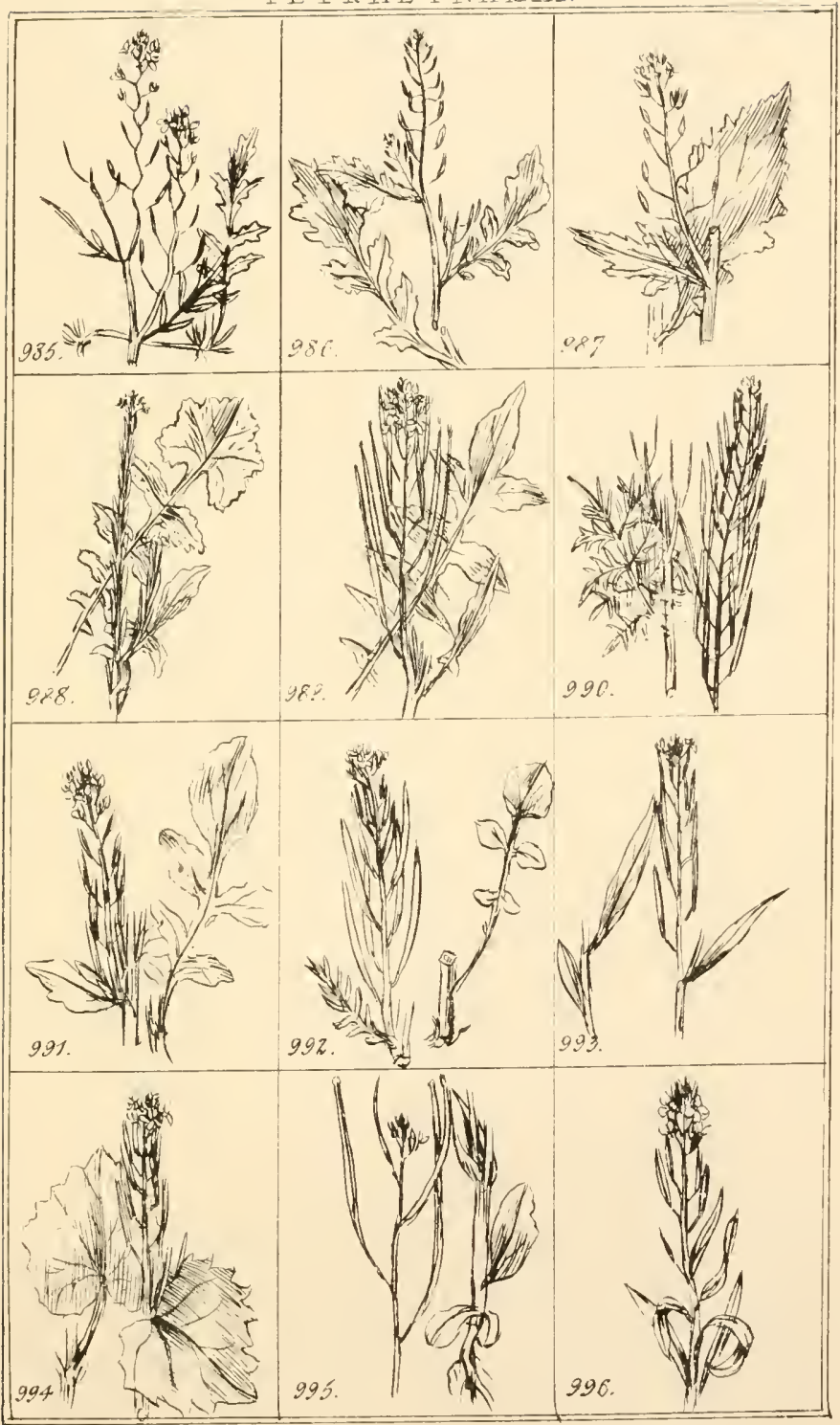





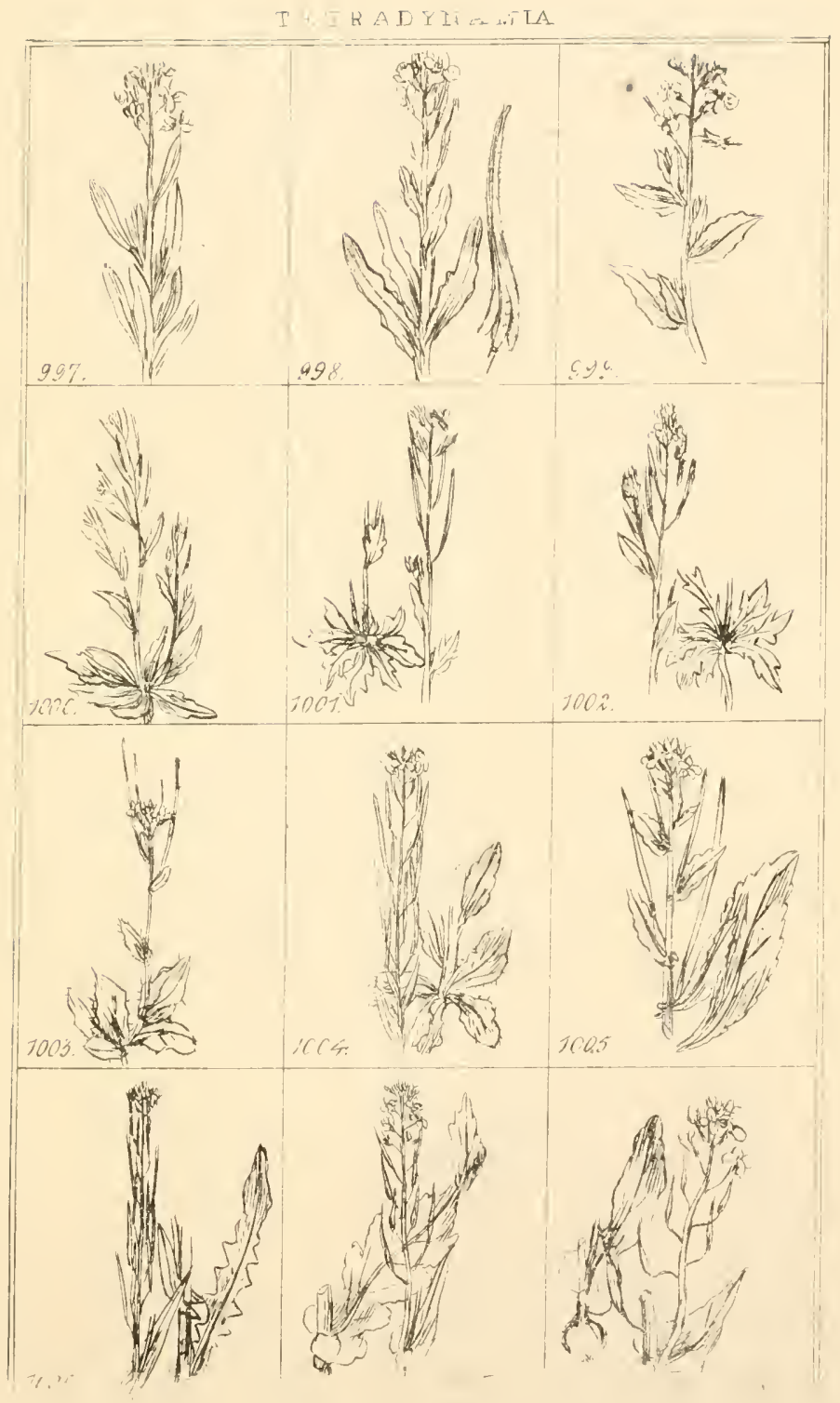



MO $15 \mathrm{~F} \perp \mathrm{PH} I \mathrm{~A}$

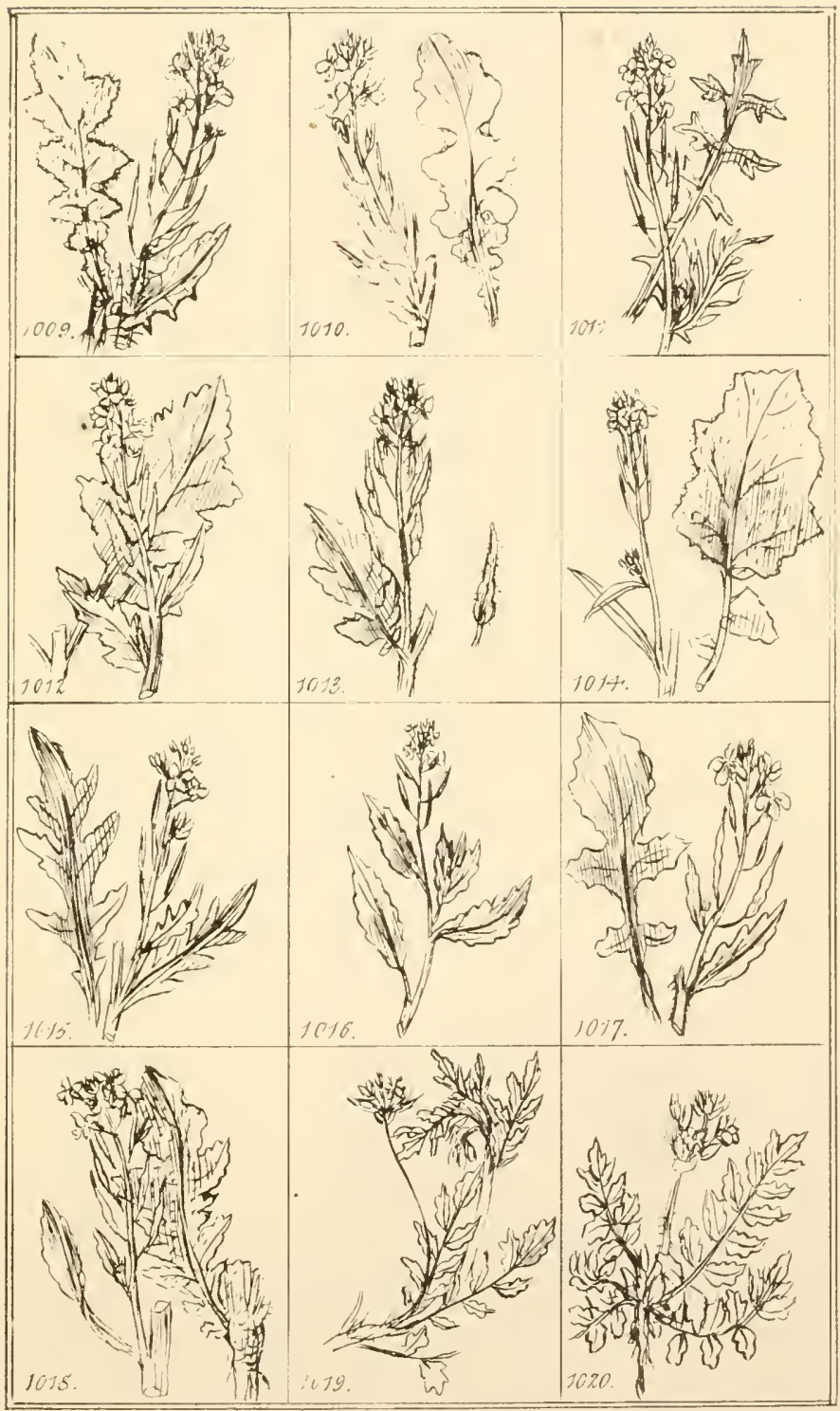





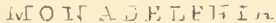

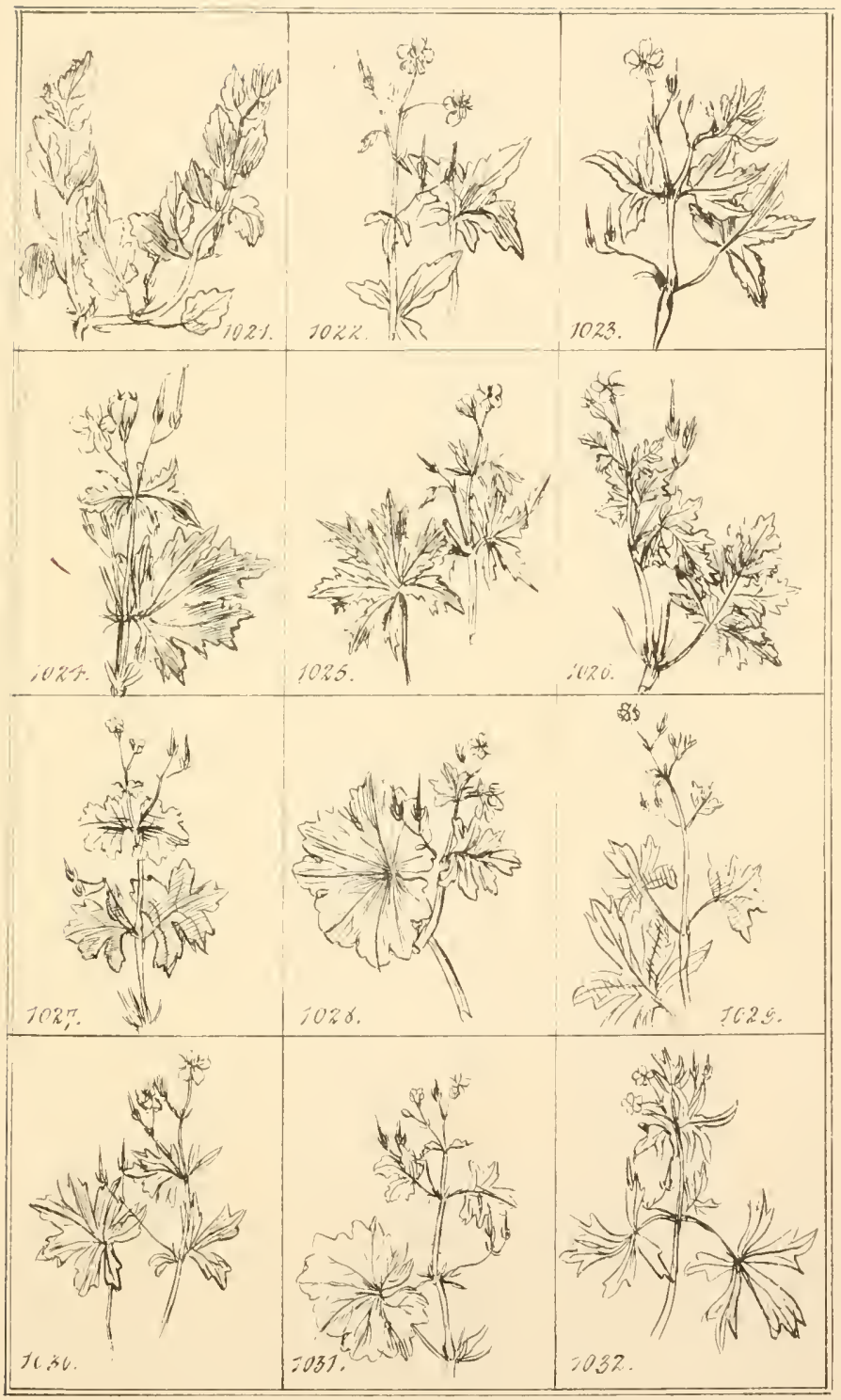



DI A DELPHIA

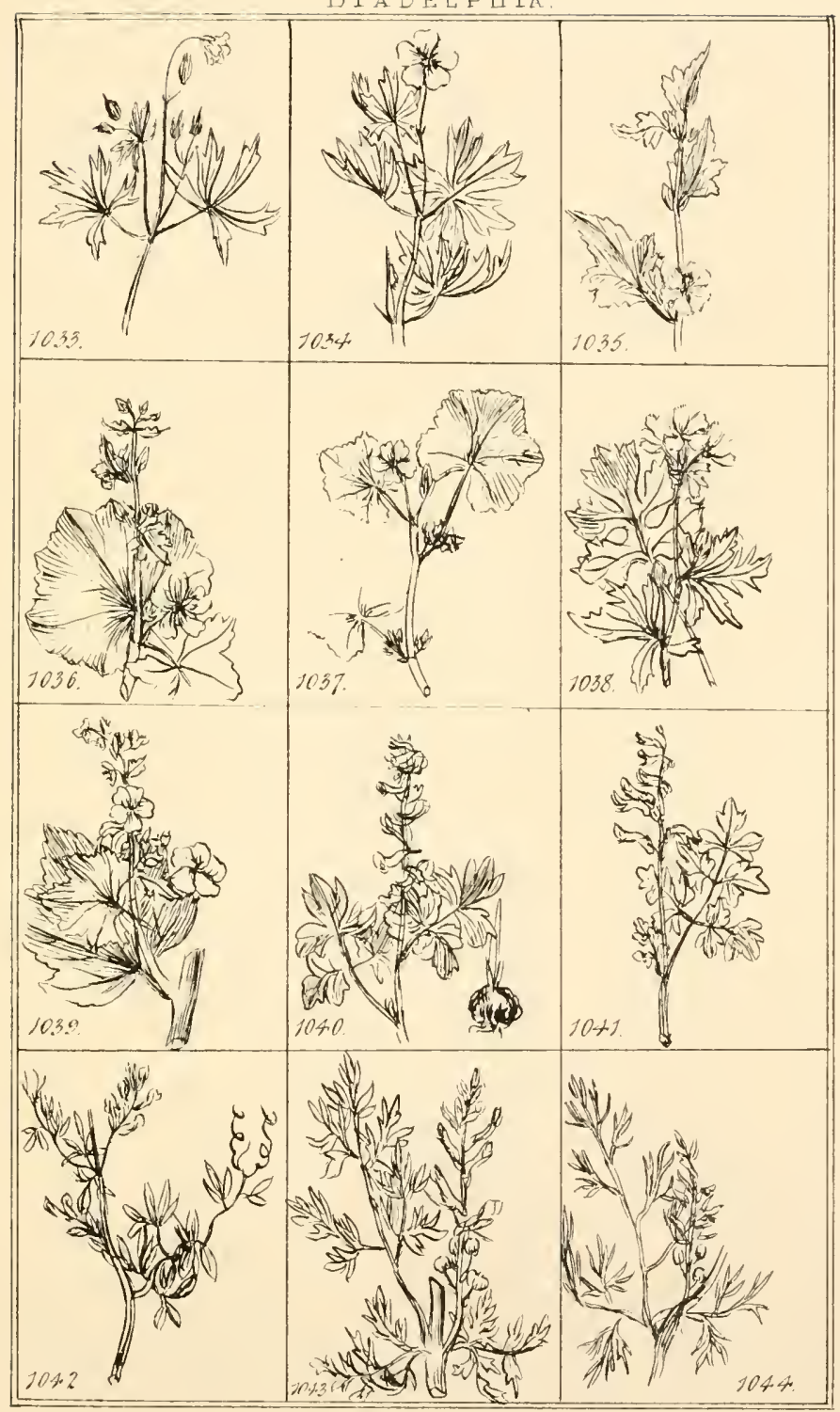



J) I A D E I P IF A

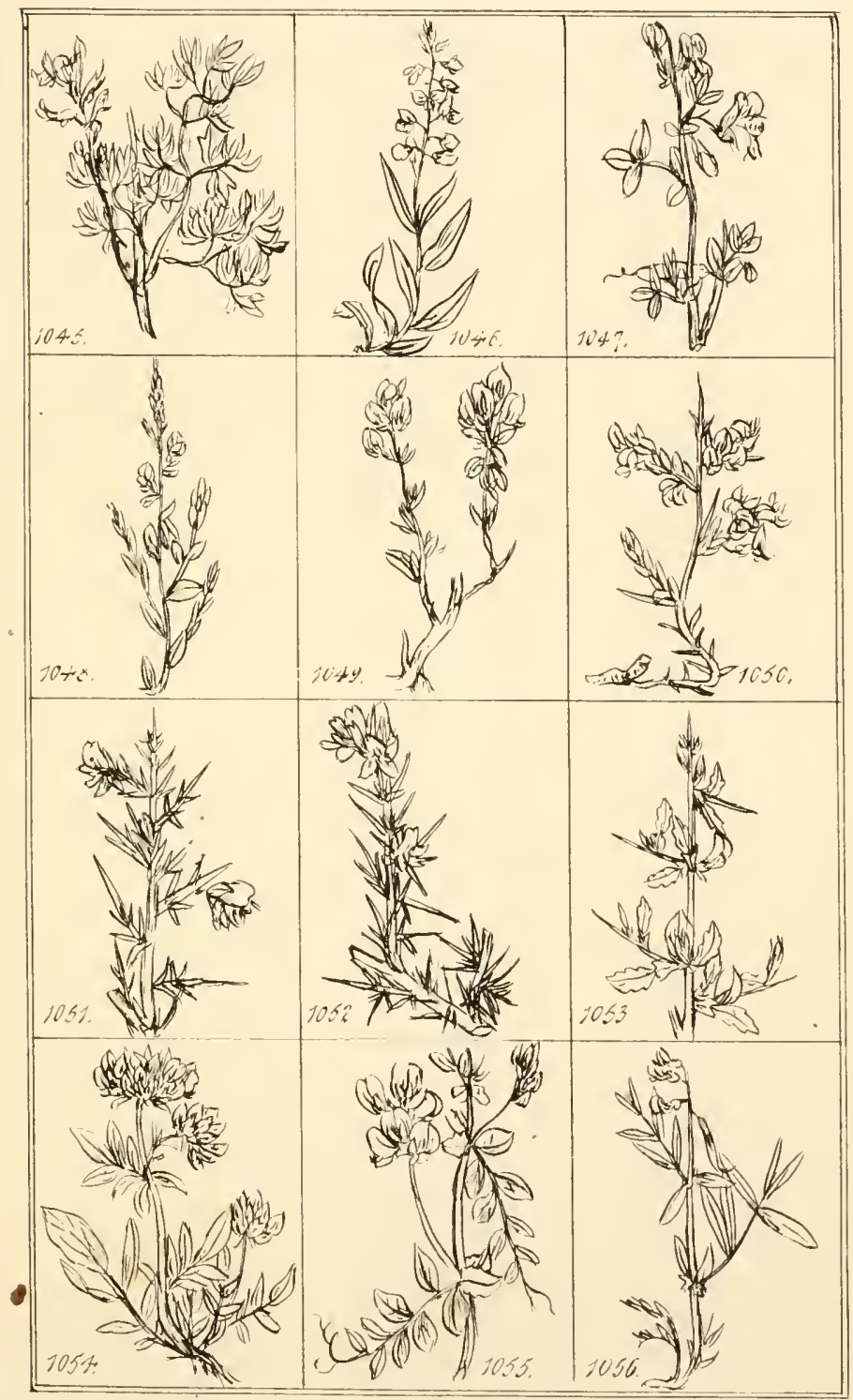





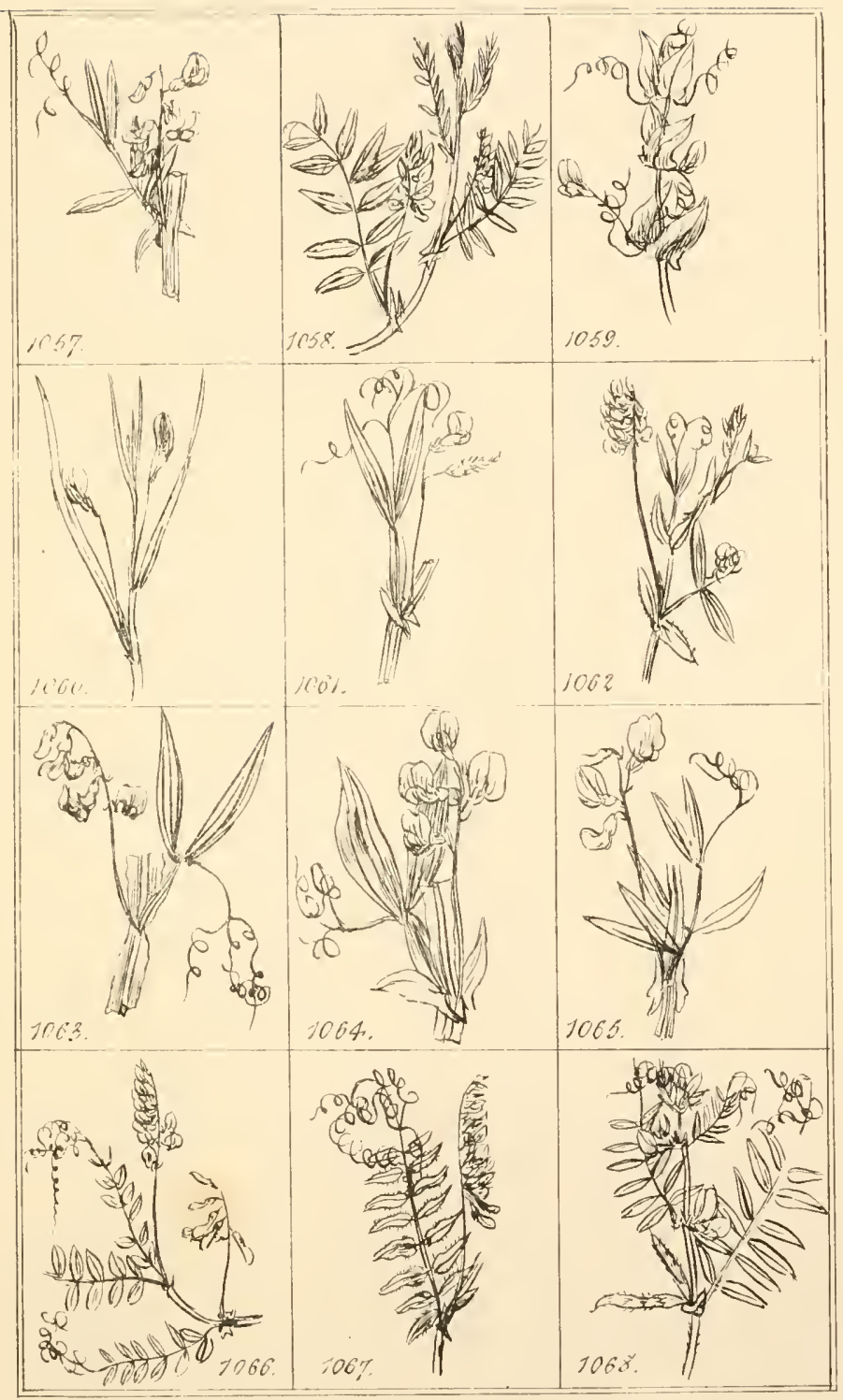





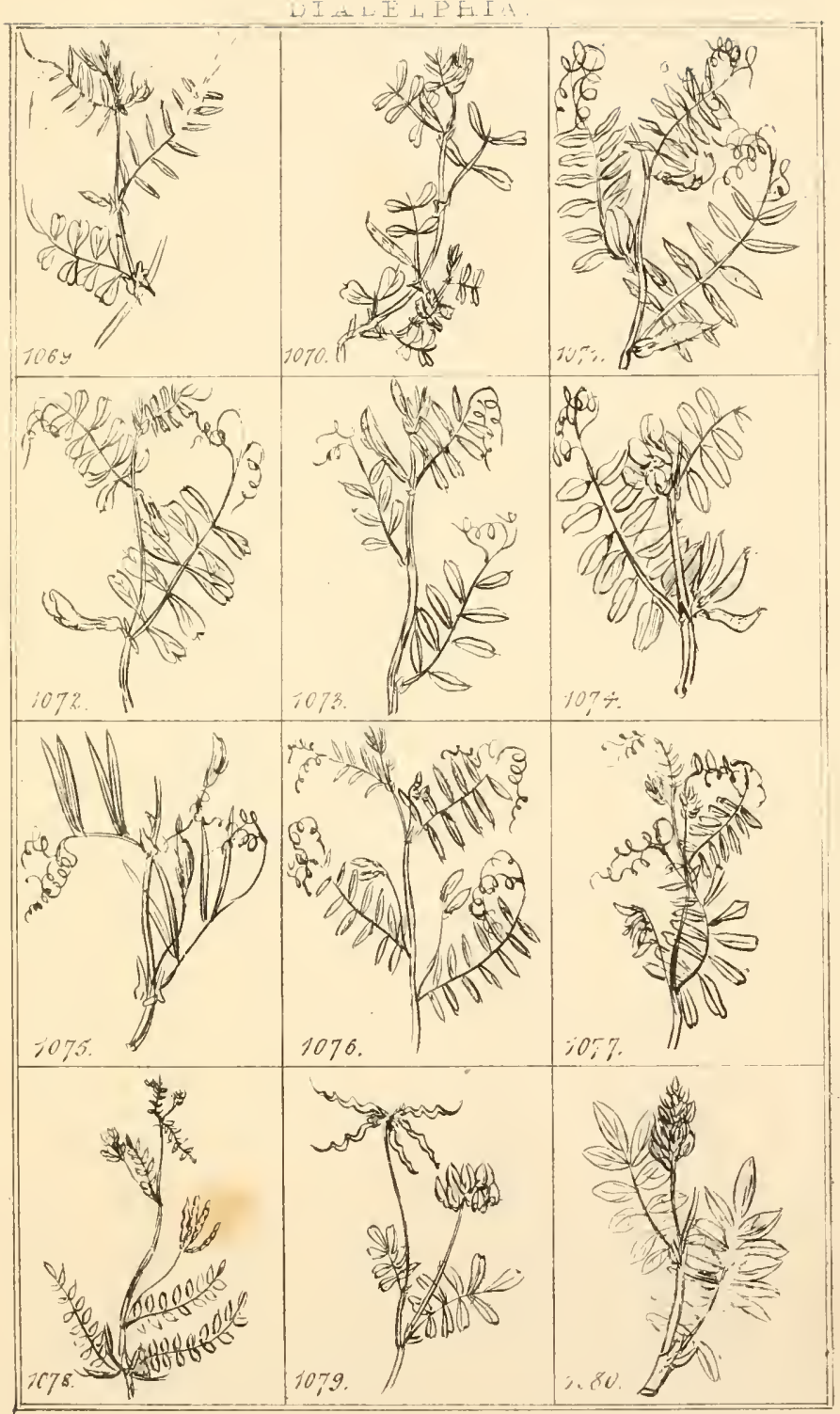



UIA D F P H IA

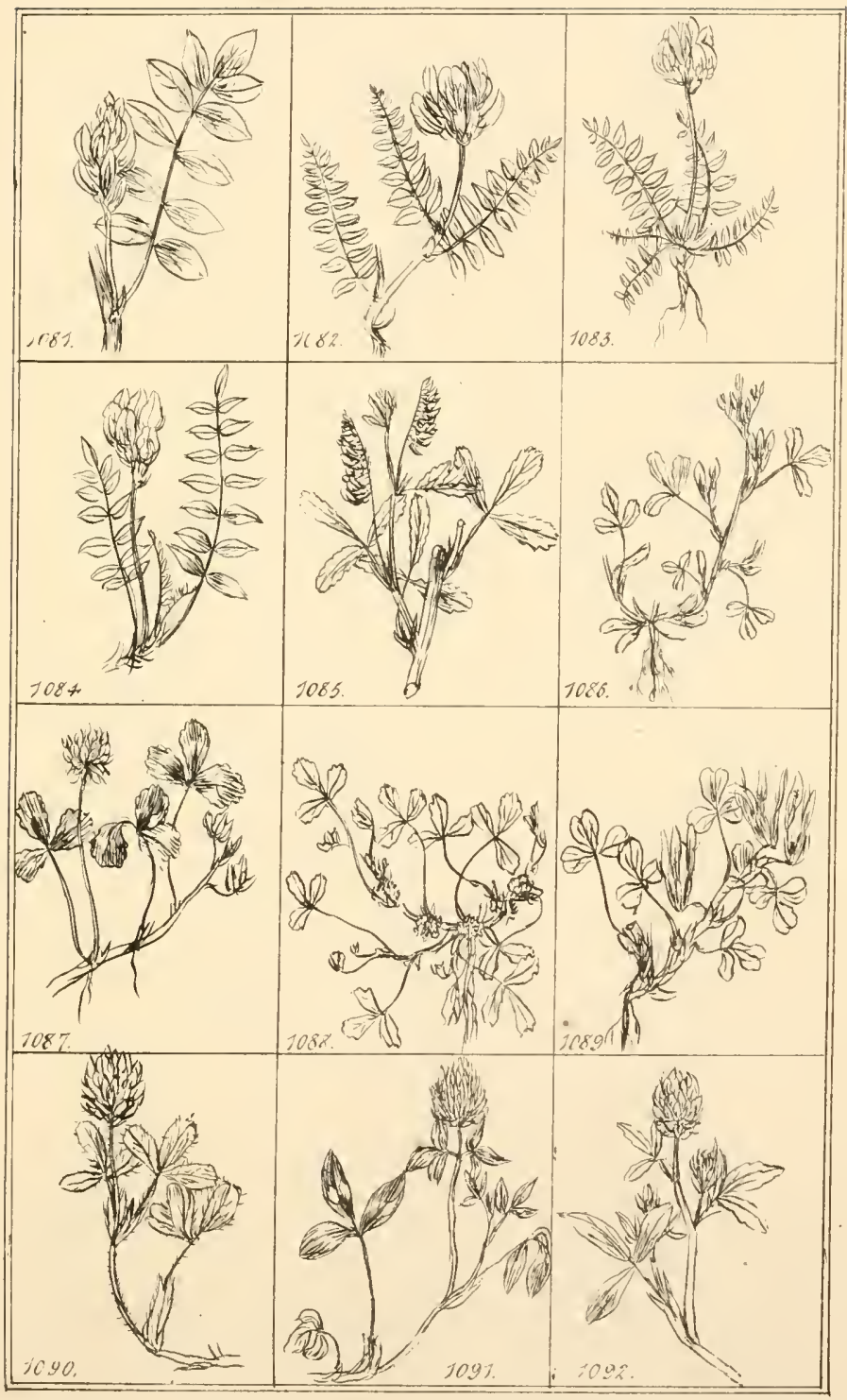



D) IA D E T P F I A

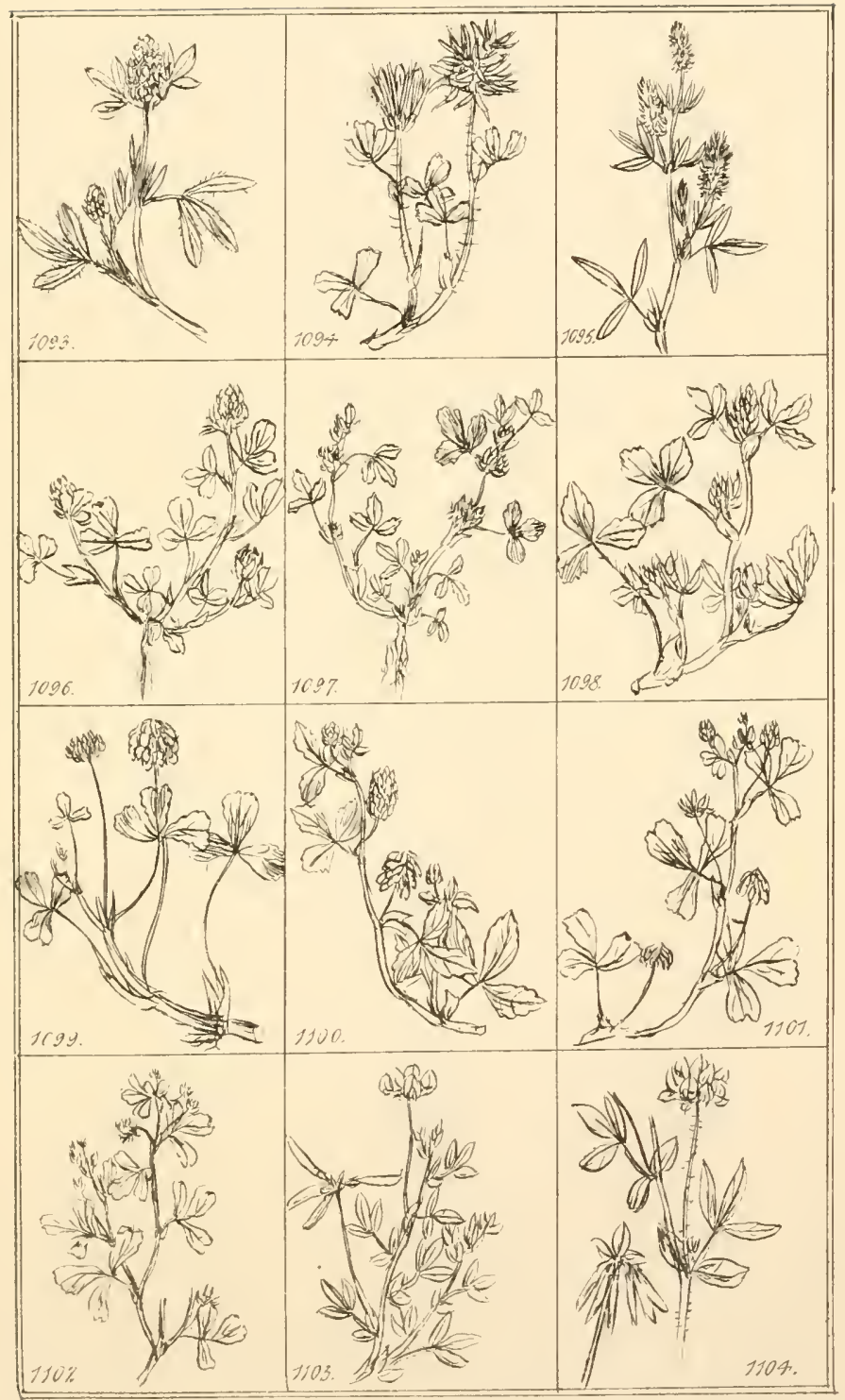





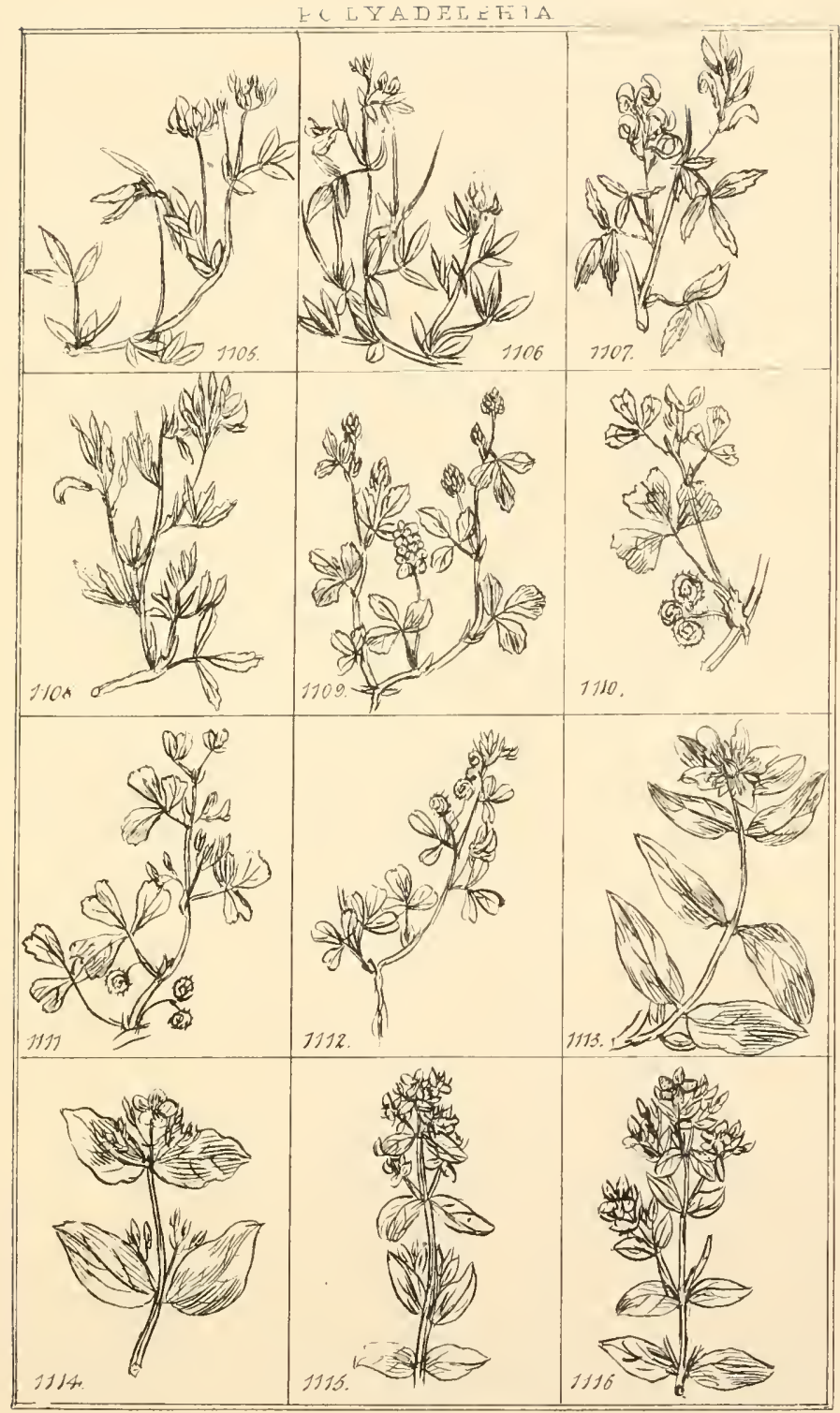



SINUENE \& IA

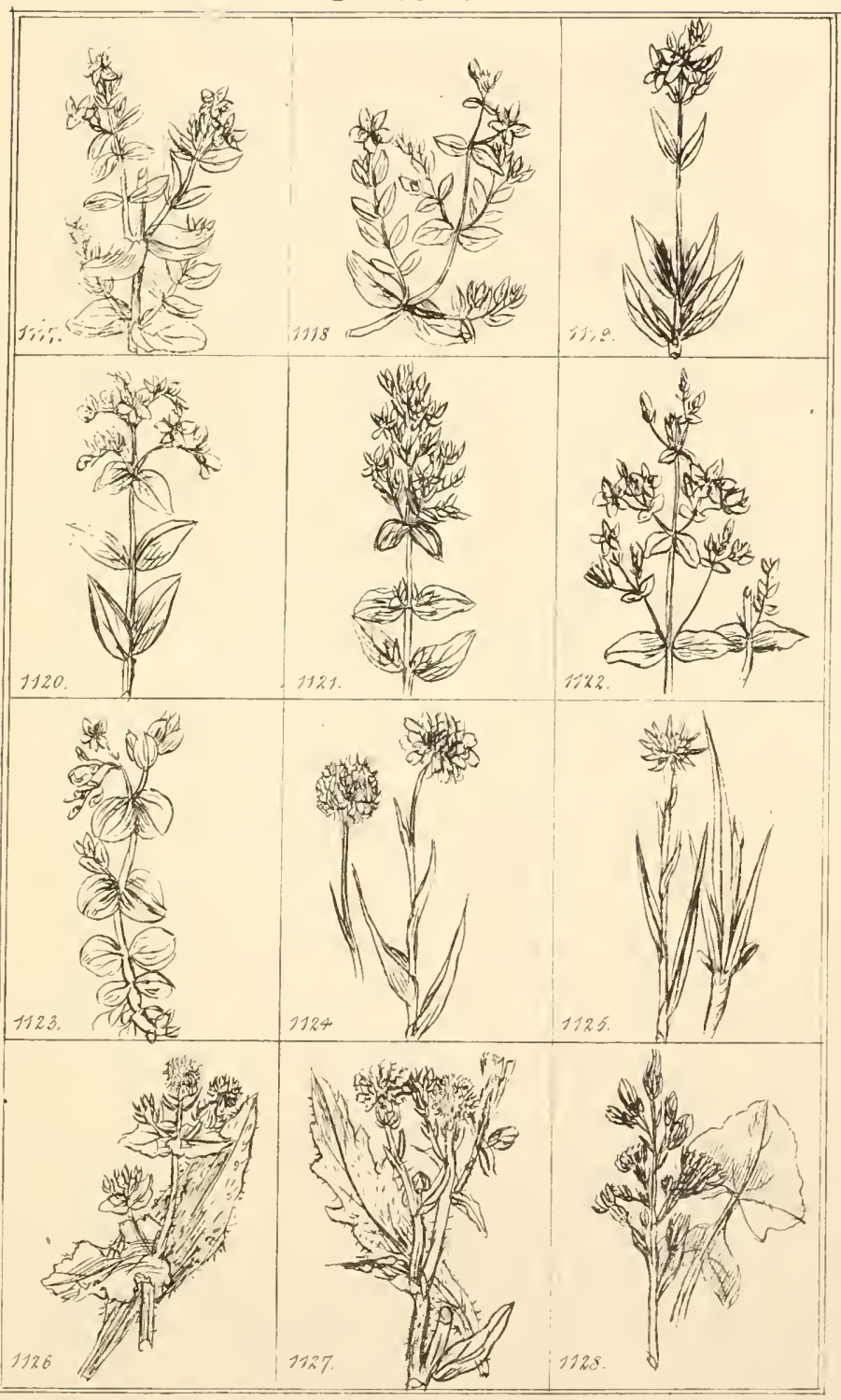





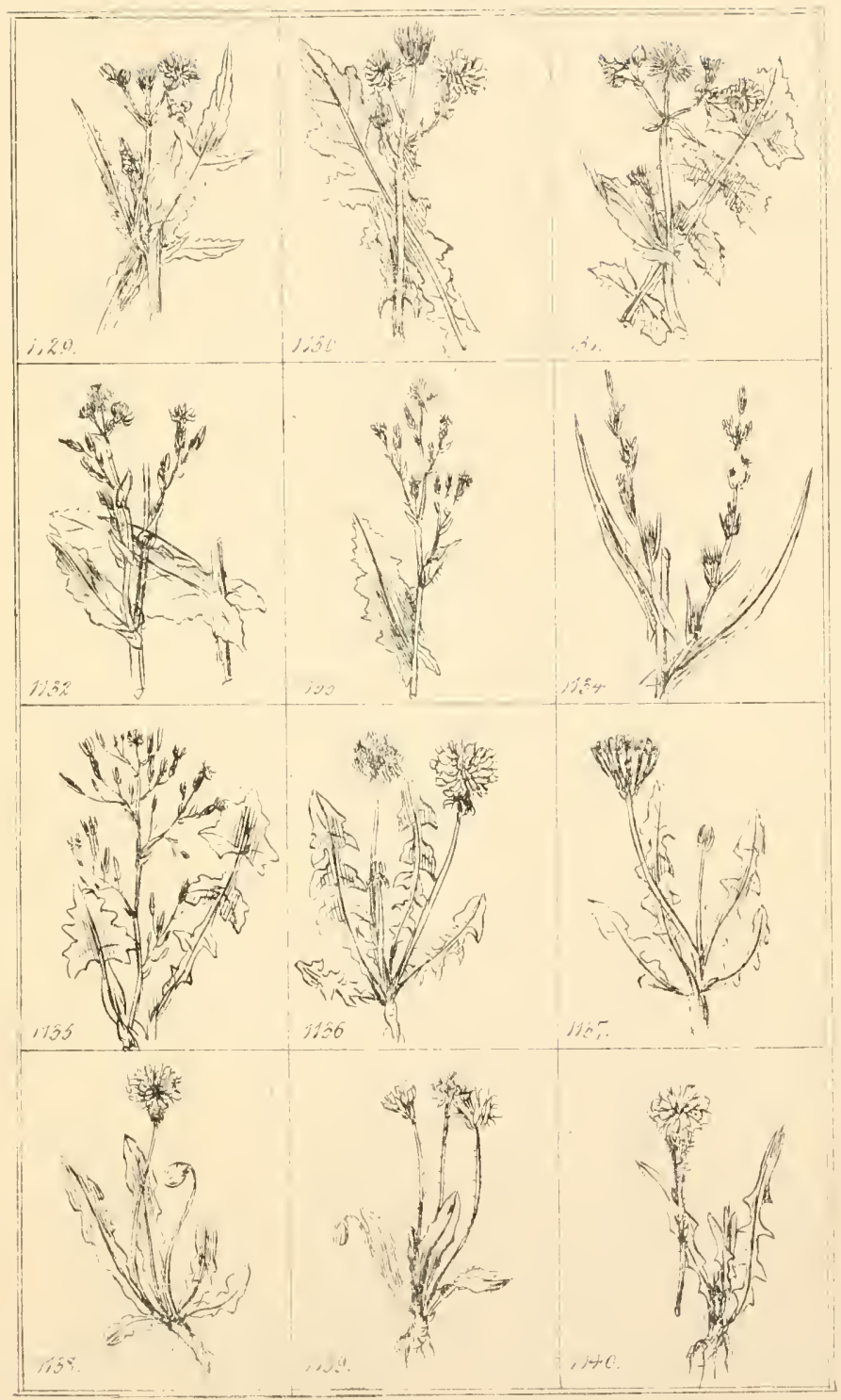





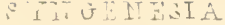

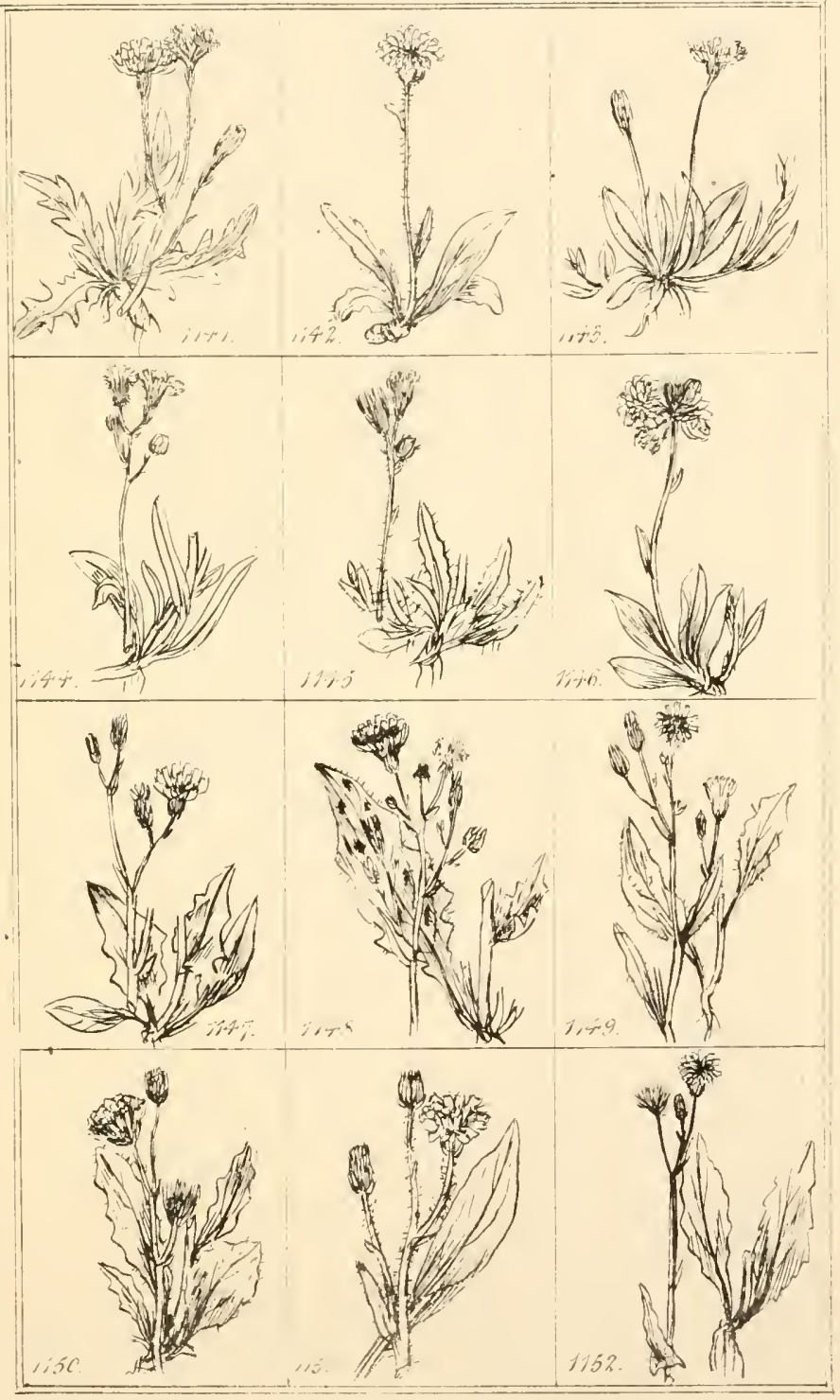



S INGENES LA.

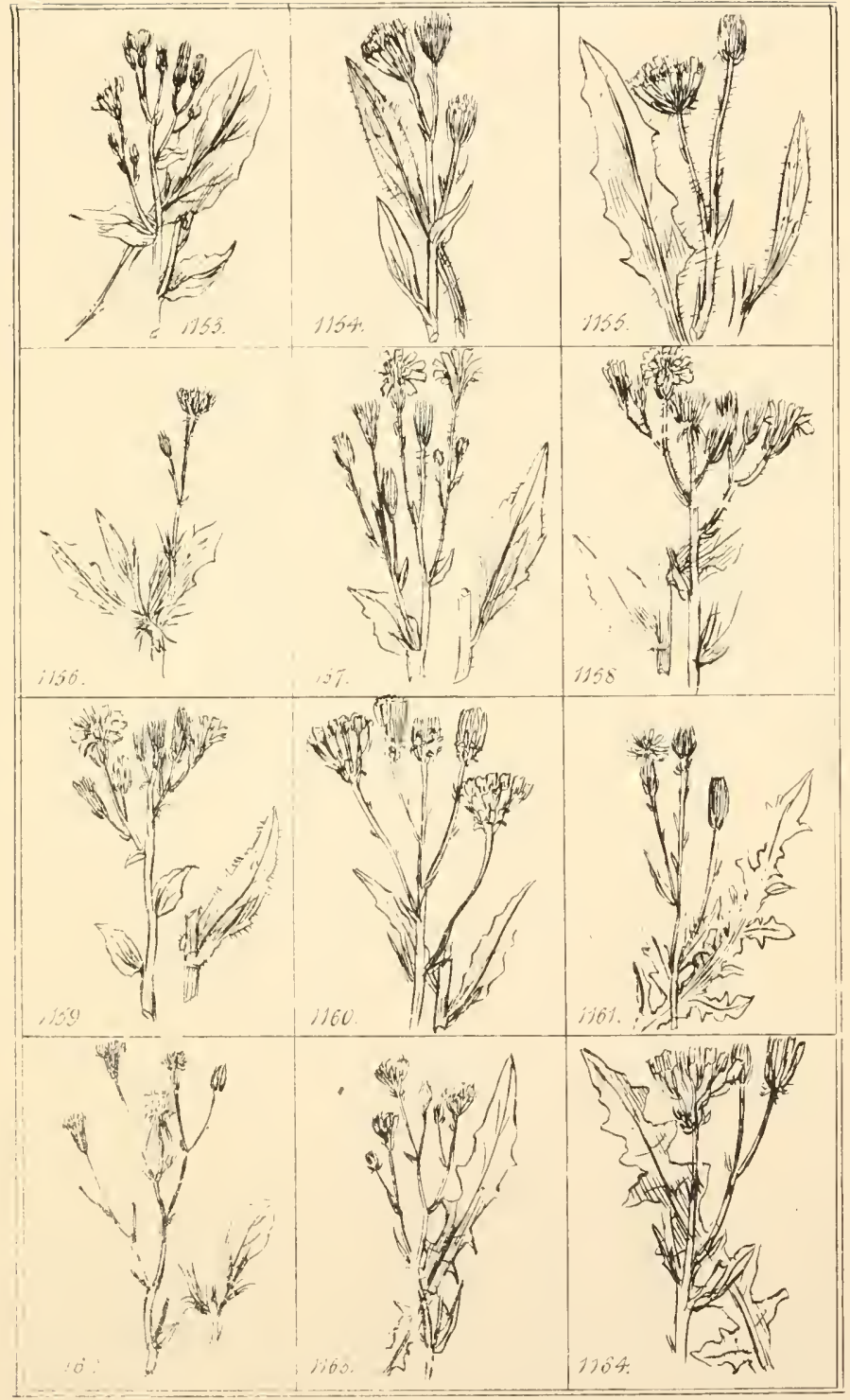





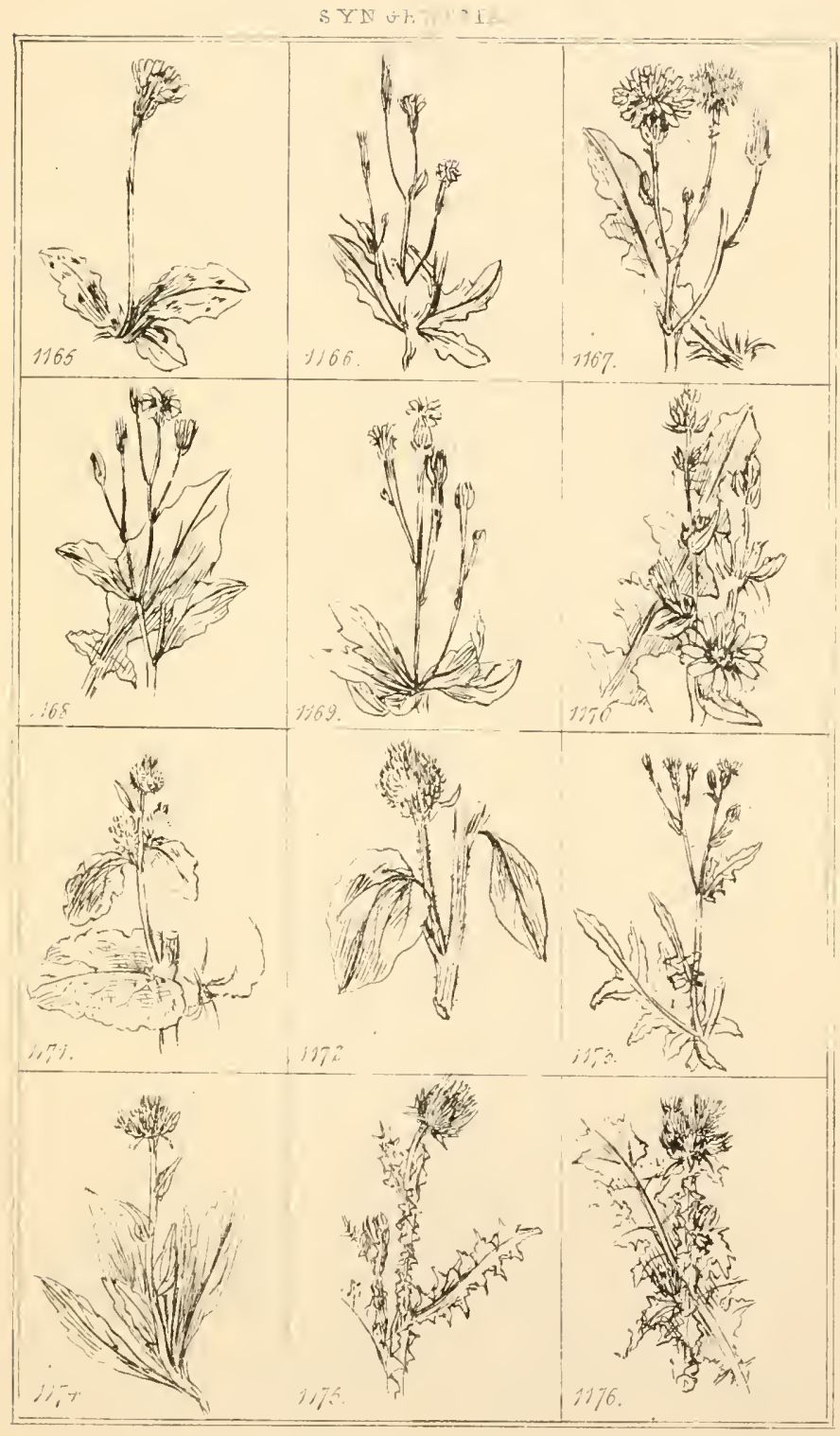



$\triangle Y N G E N E S I A$.

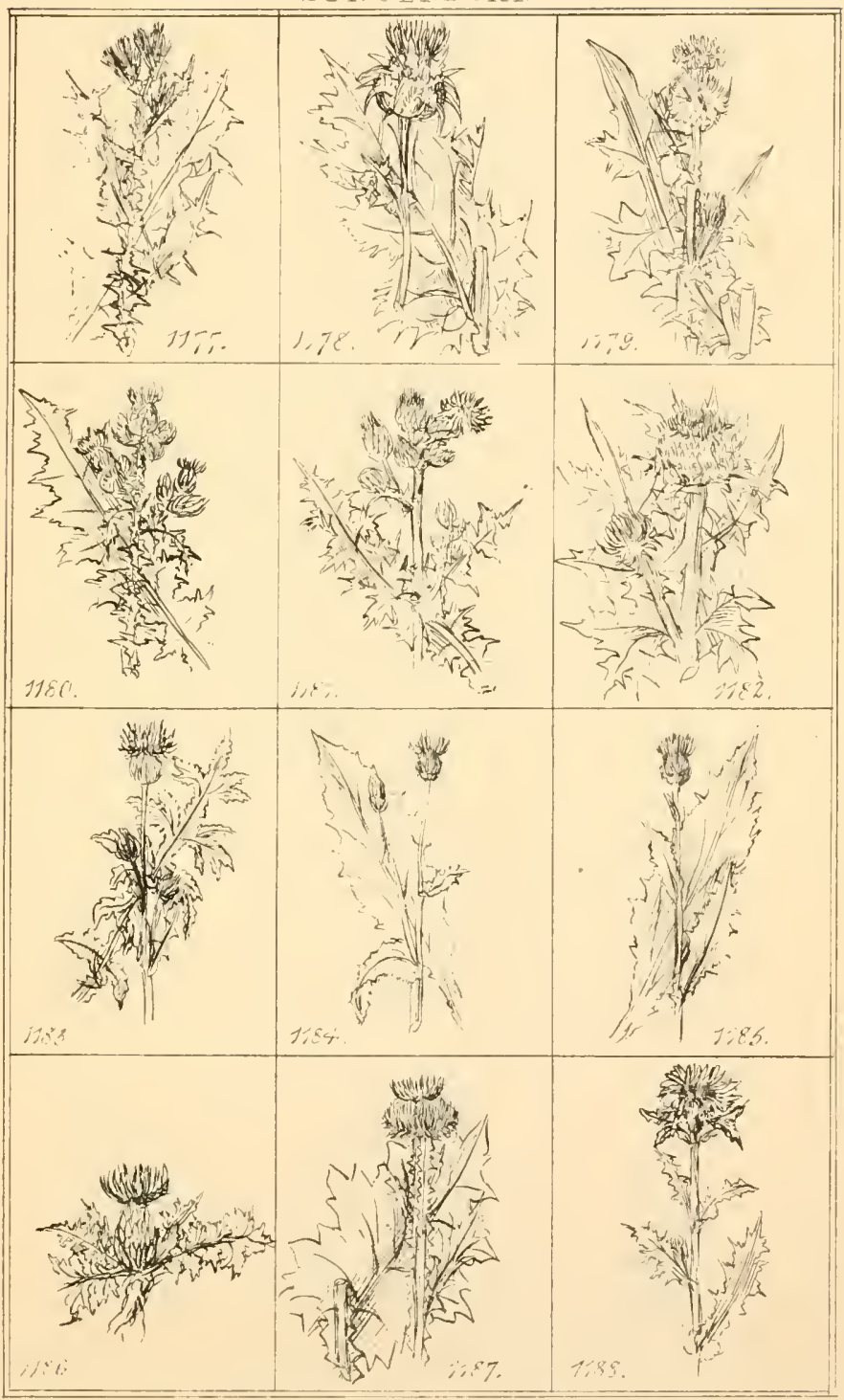



SYNGENE

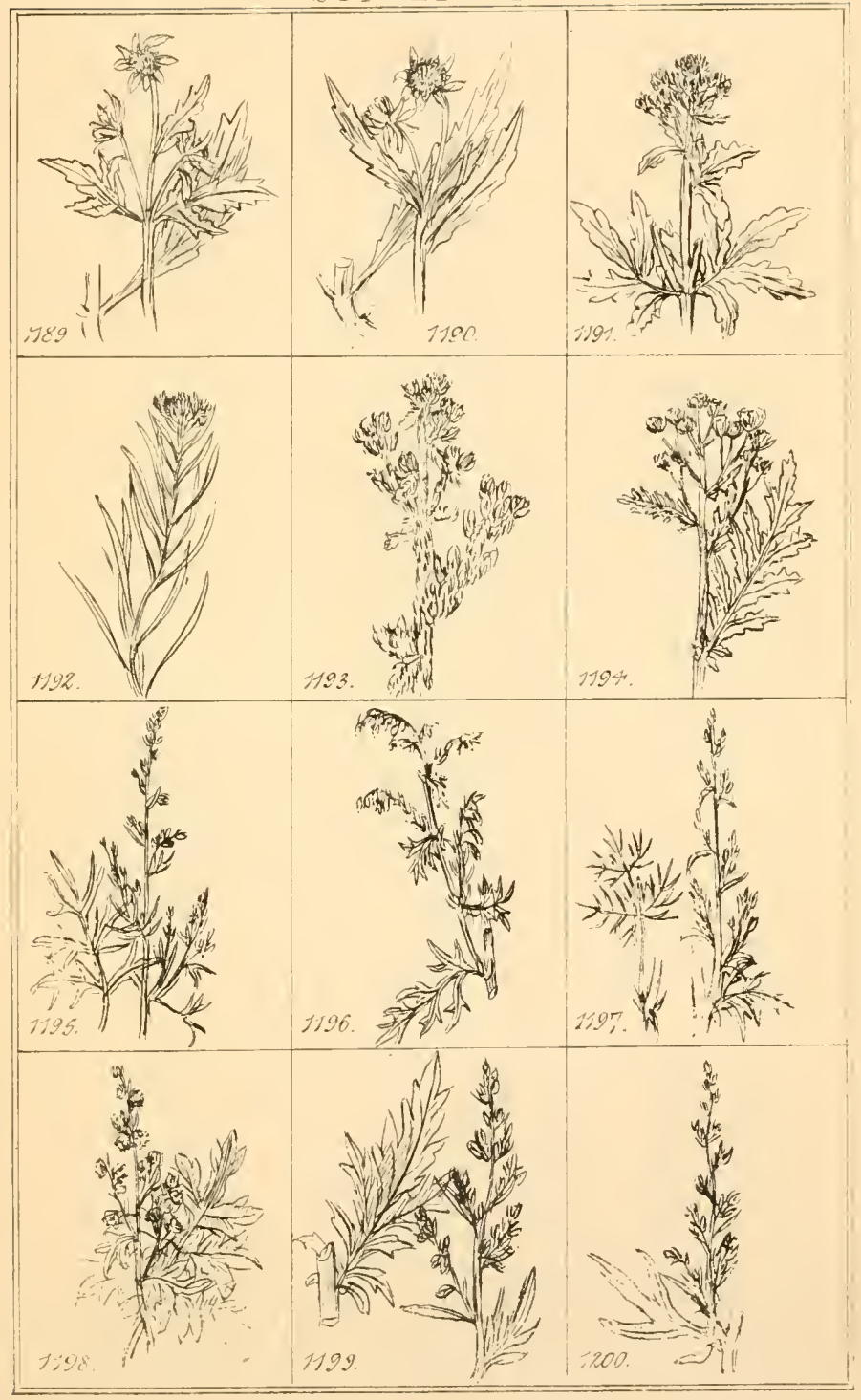



SYNGT:LNIA

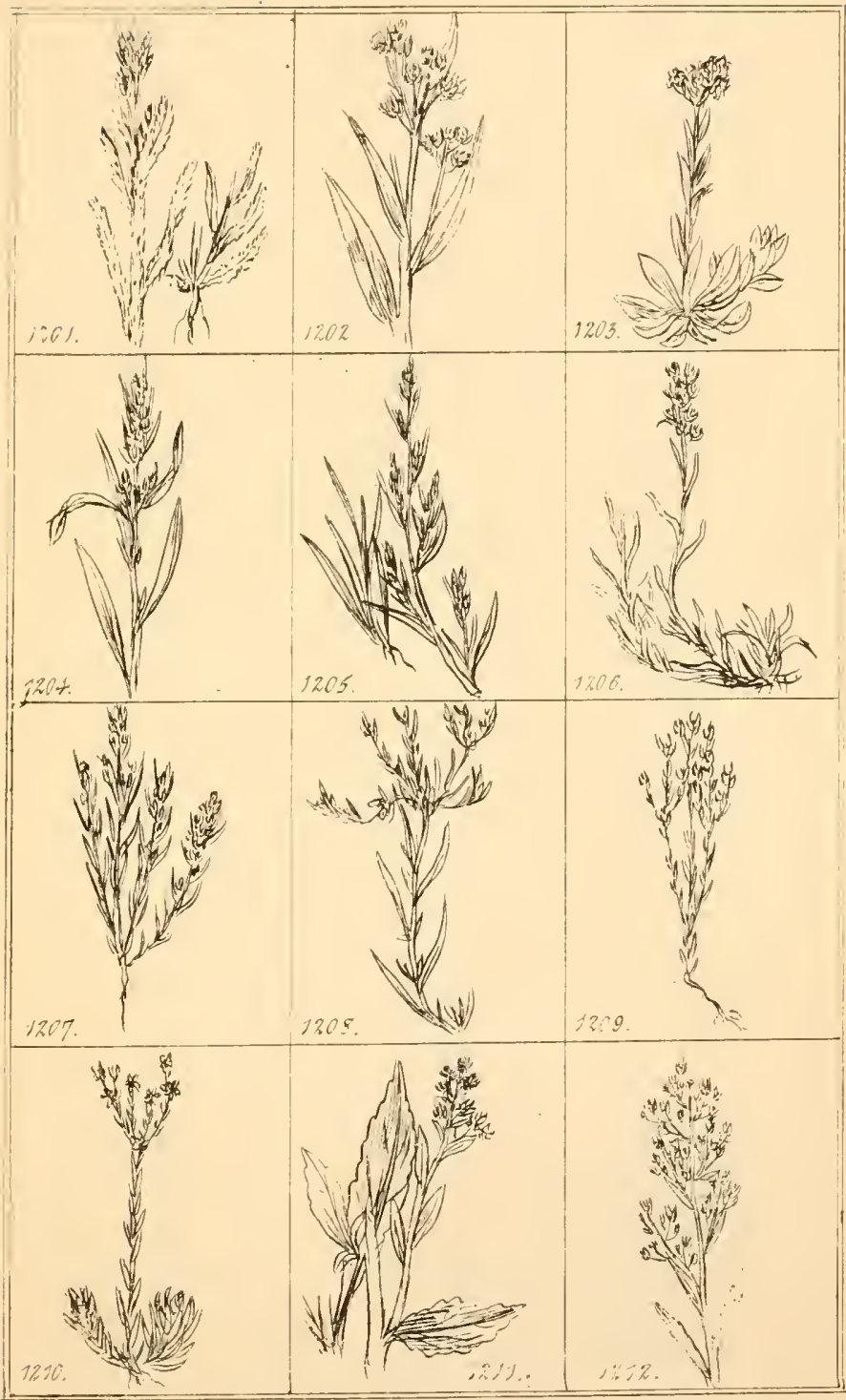



SYIVGENESIA

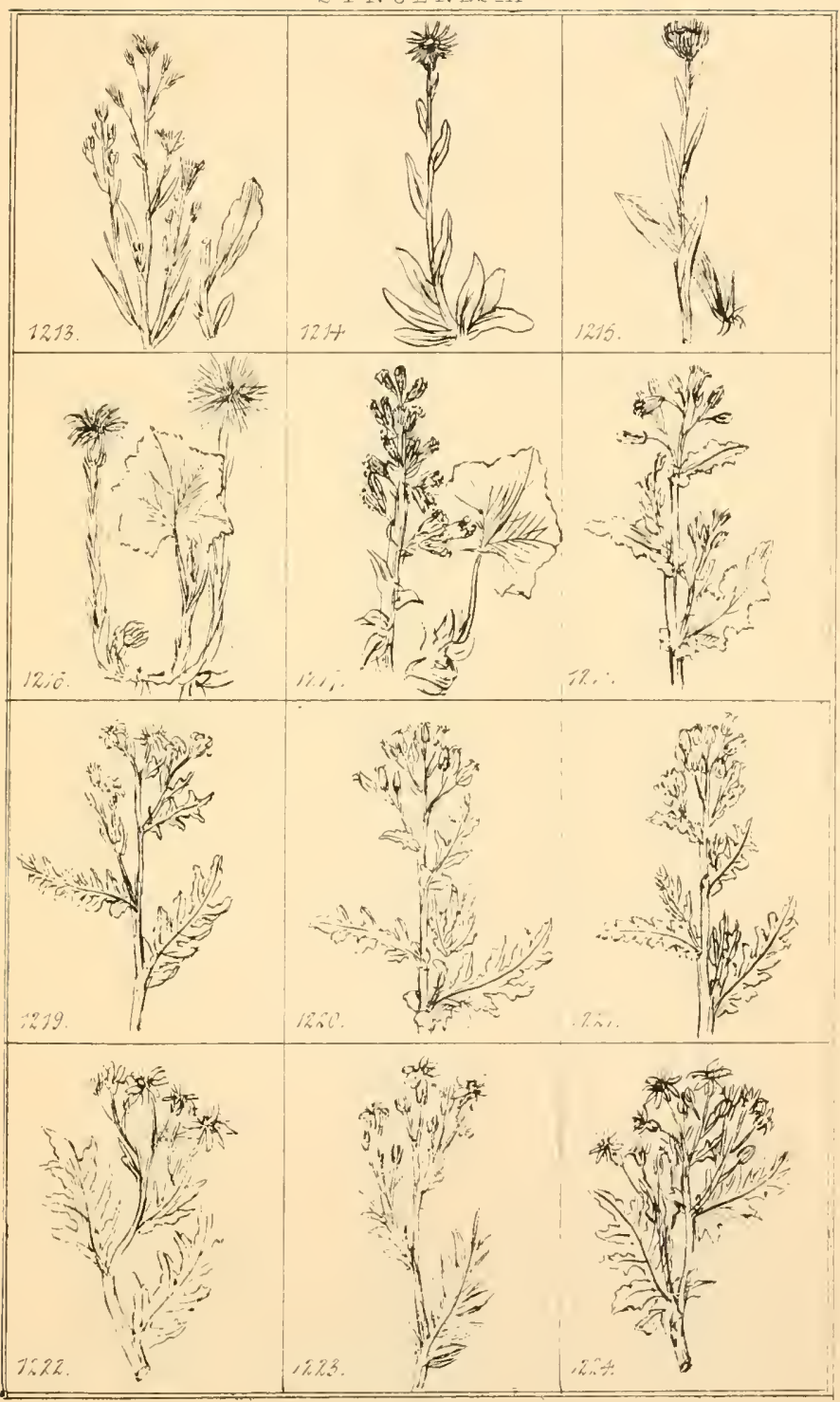



SYNEIESTA

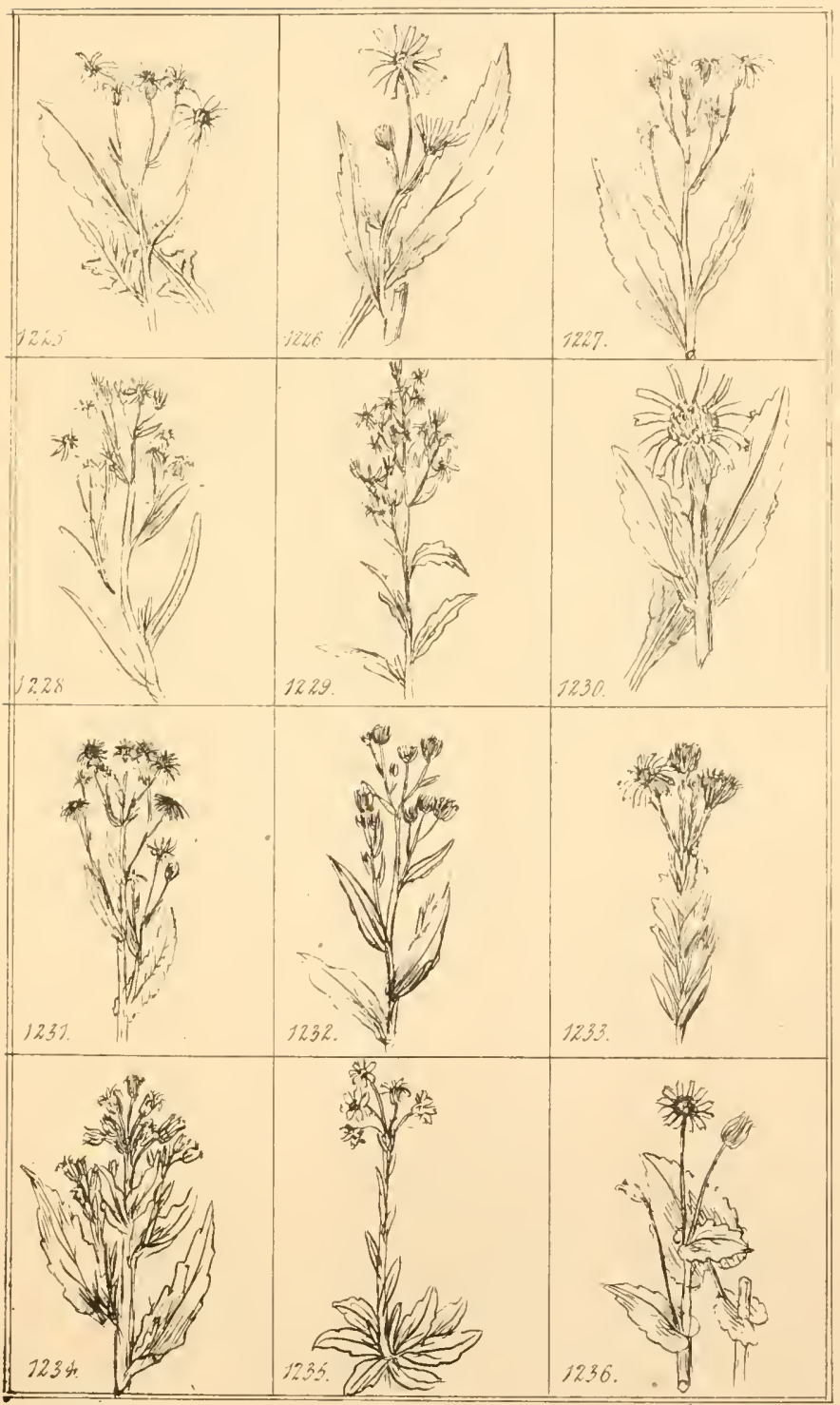





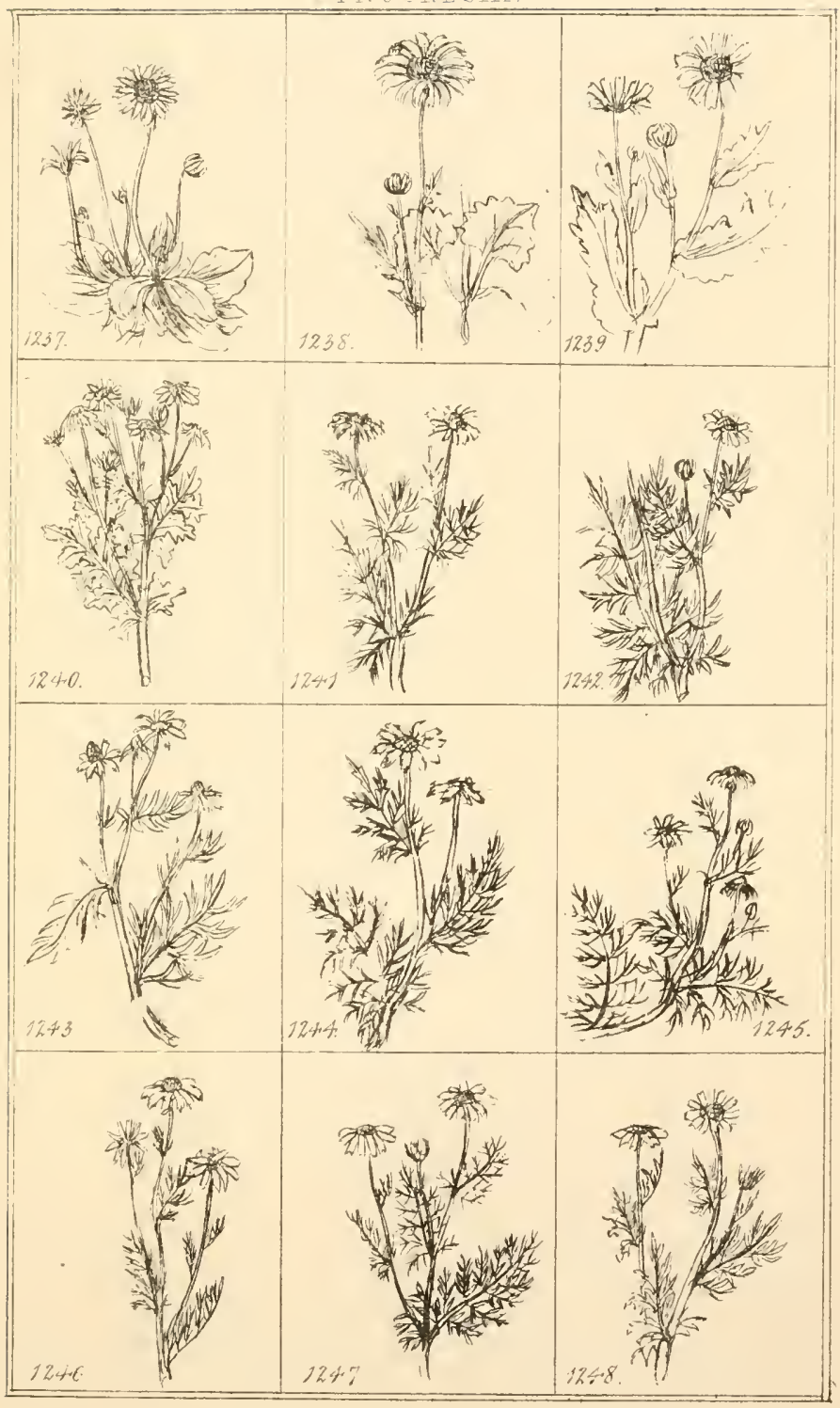



WITANDEIA

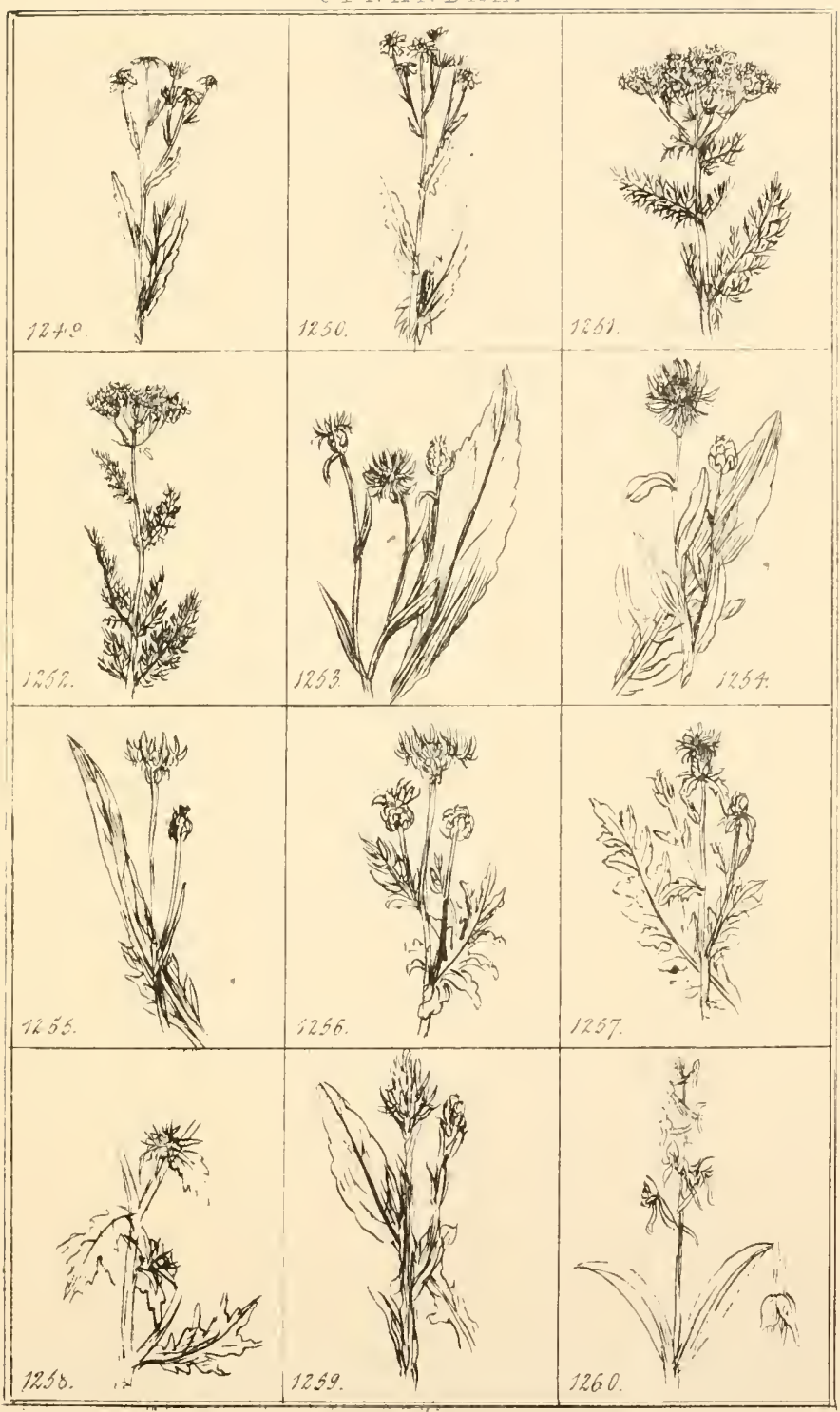



GYNANDRIA

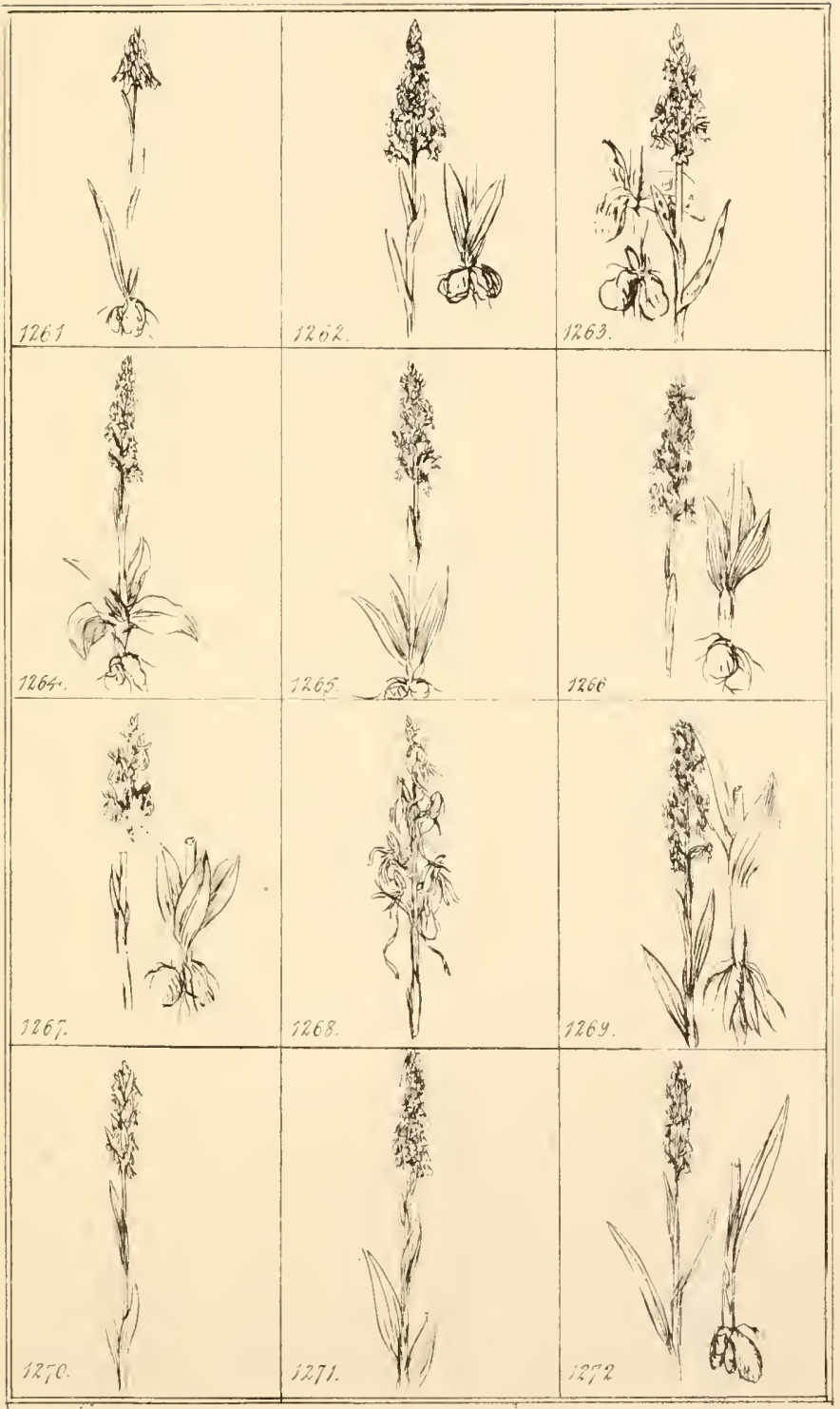





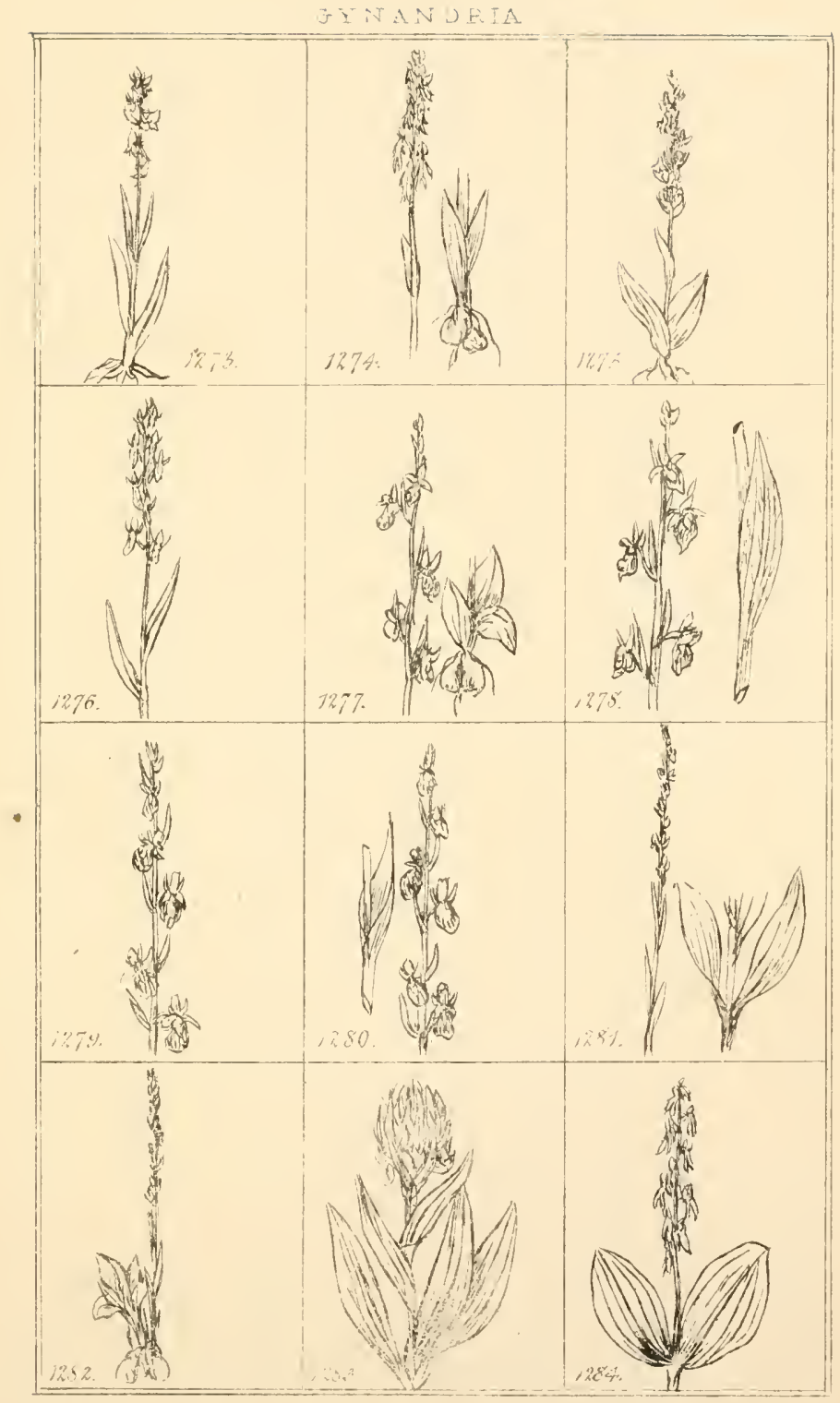





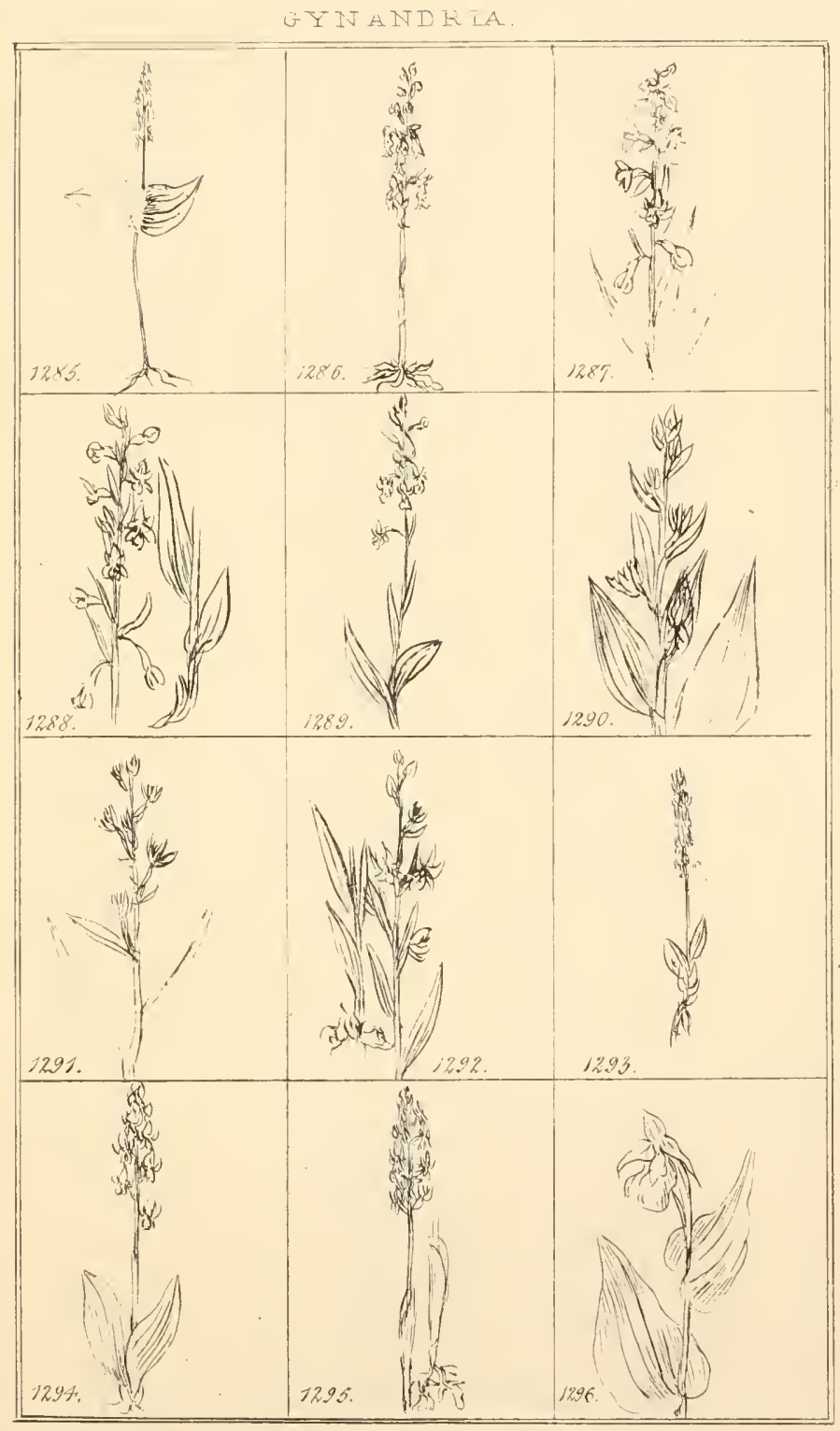



IV: 2 I $E I A$

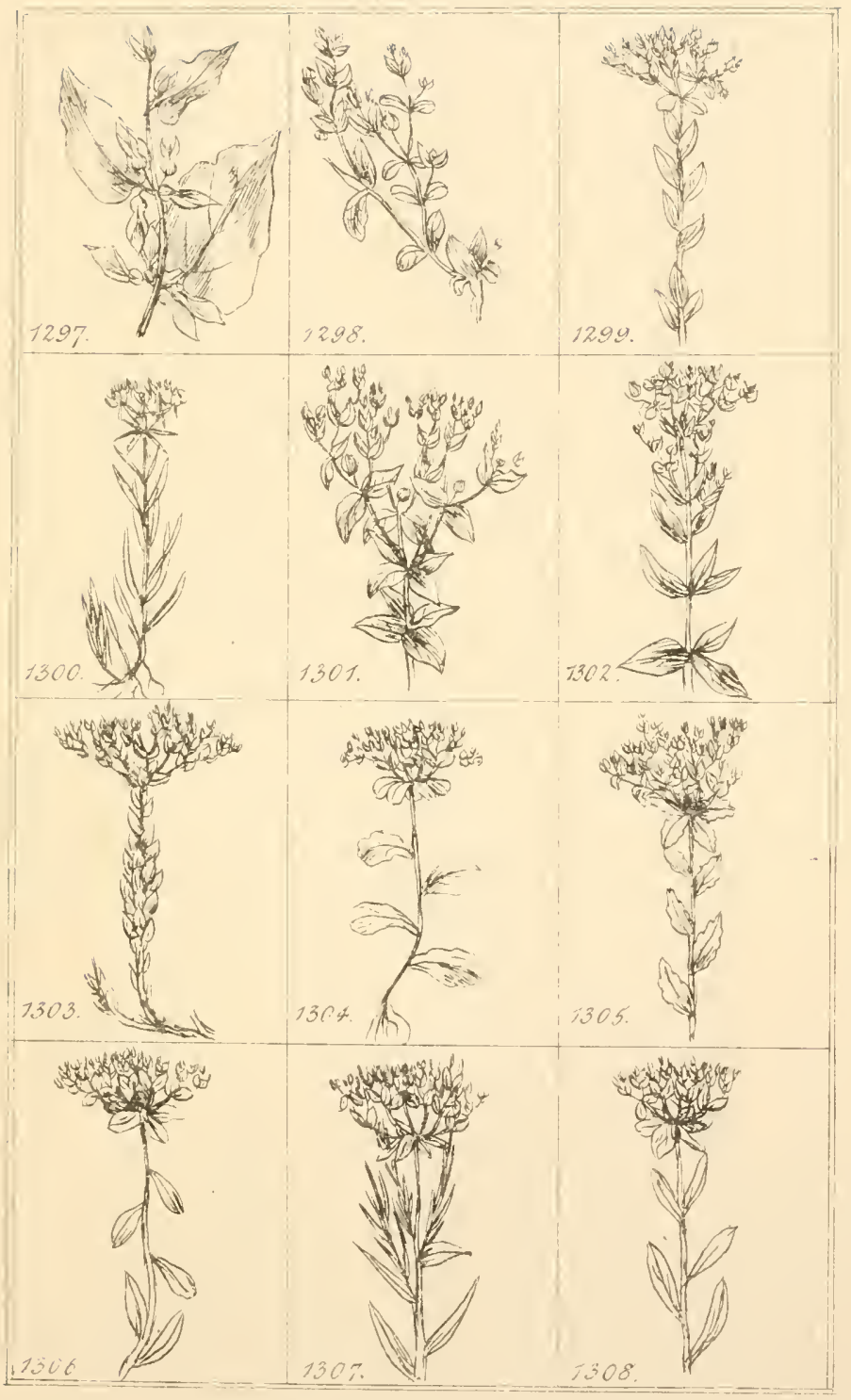





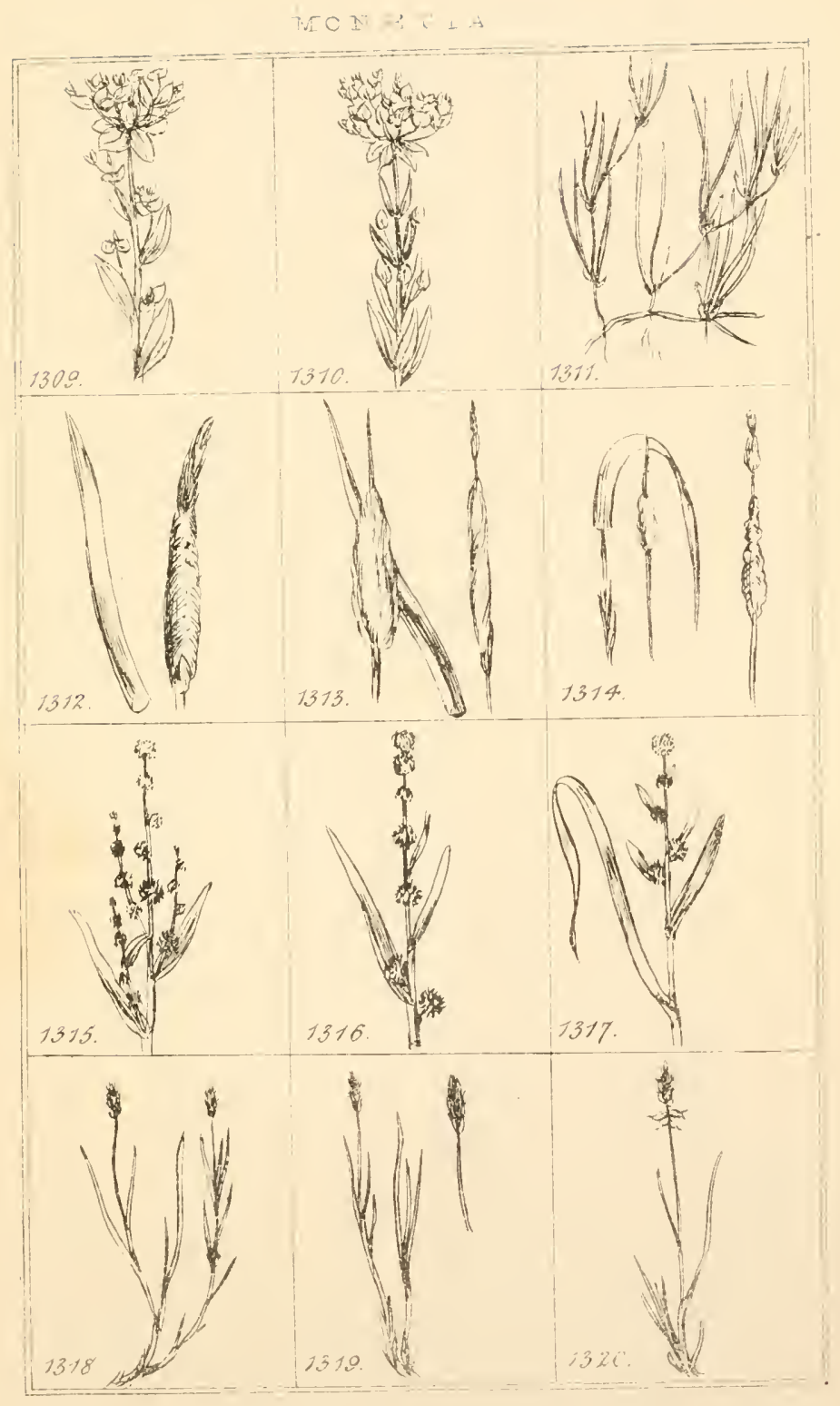



IINITECELA

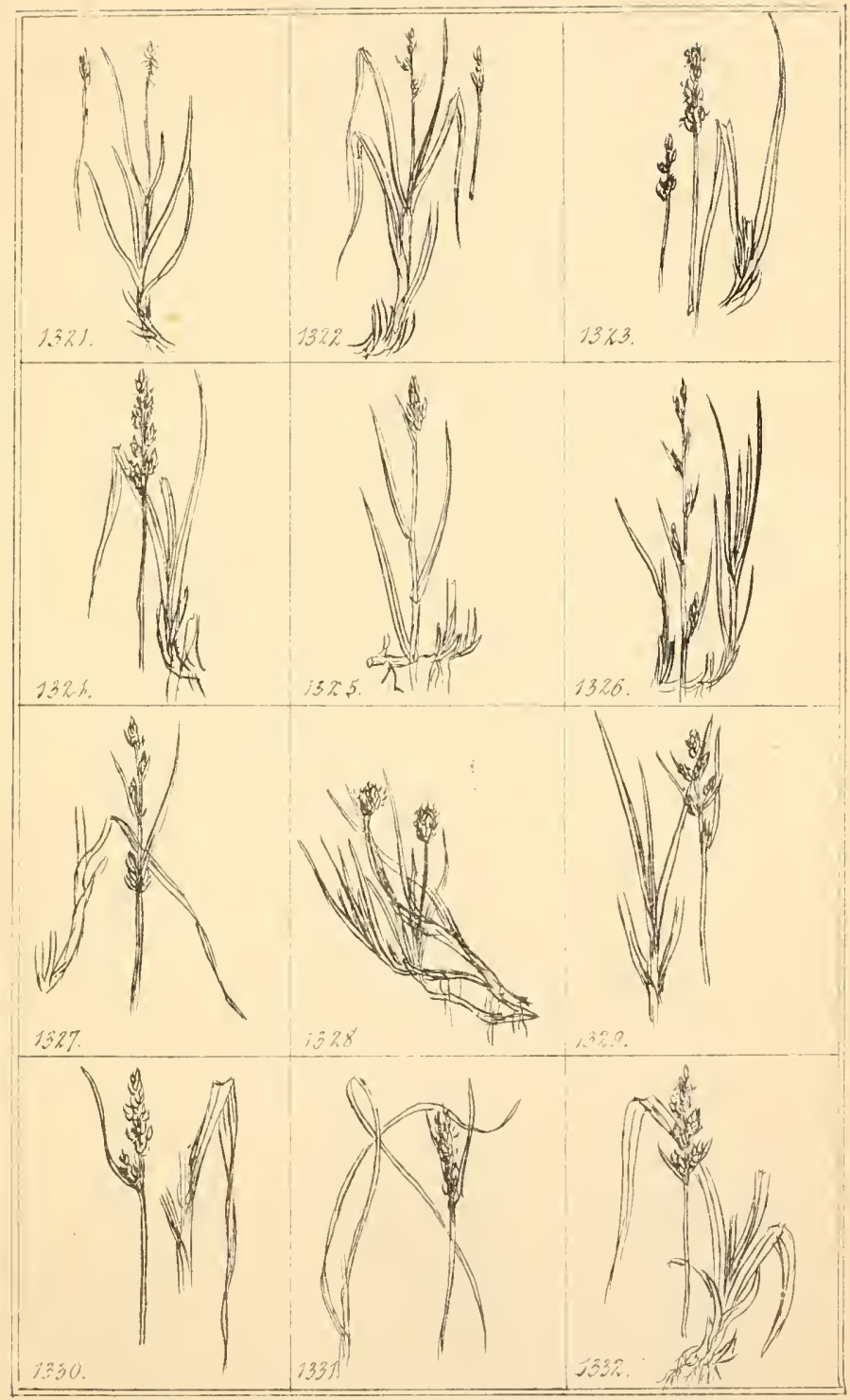



IVUNEこEIA

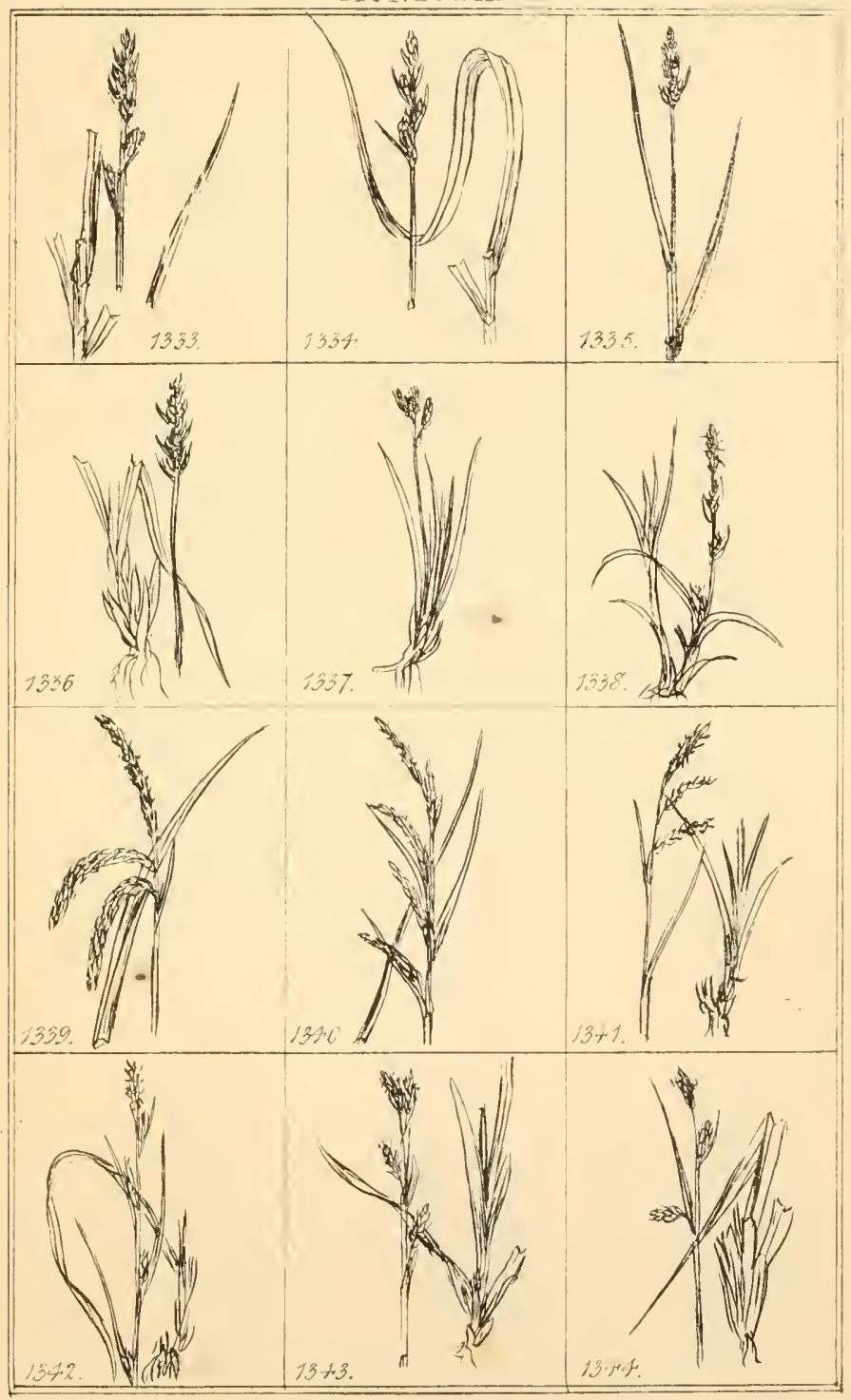



ICIJEこEIA

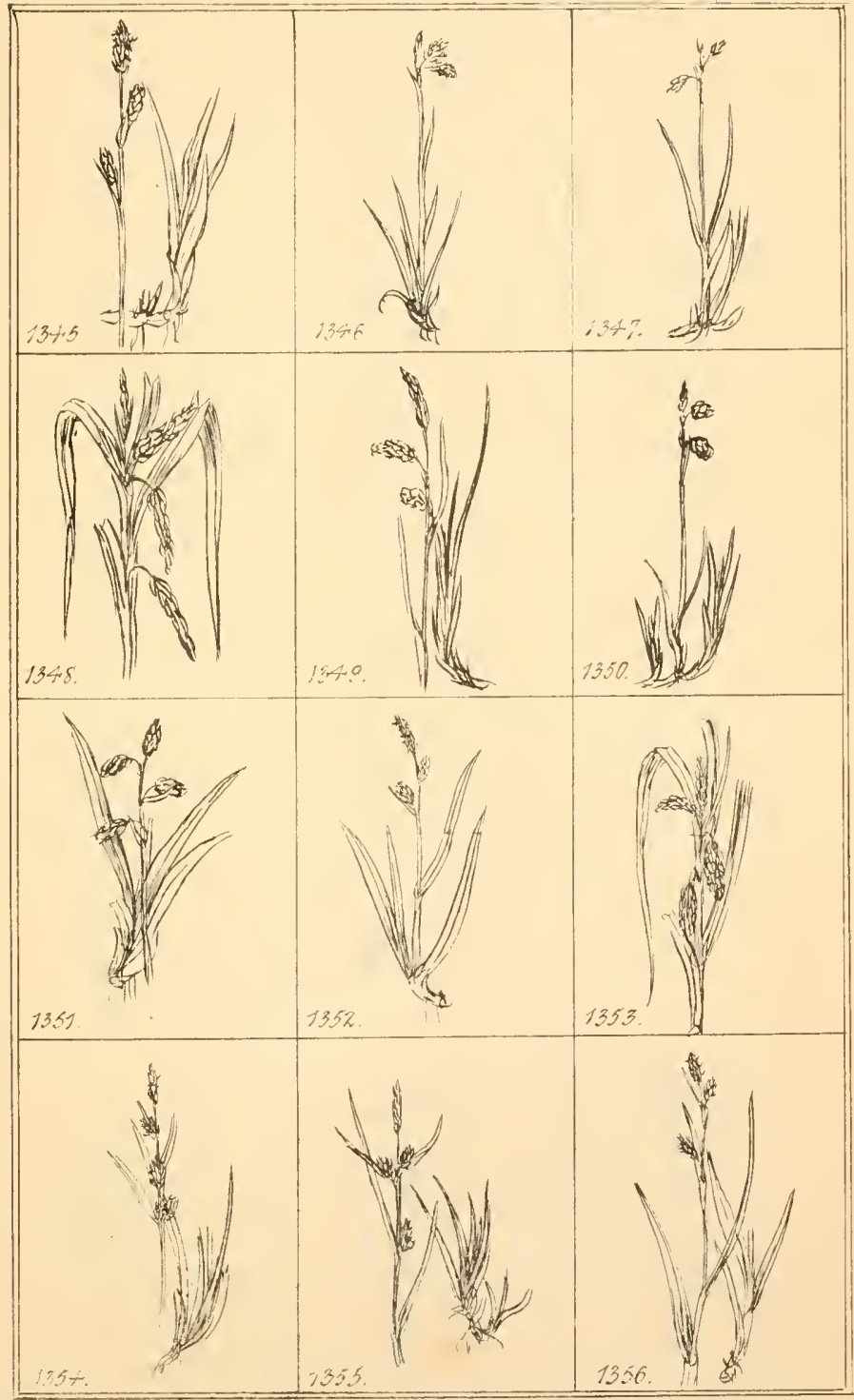



IVO NECEIA

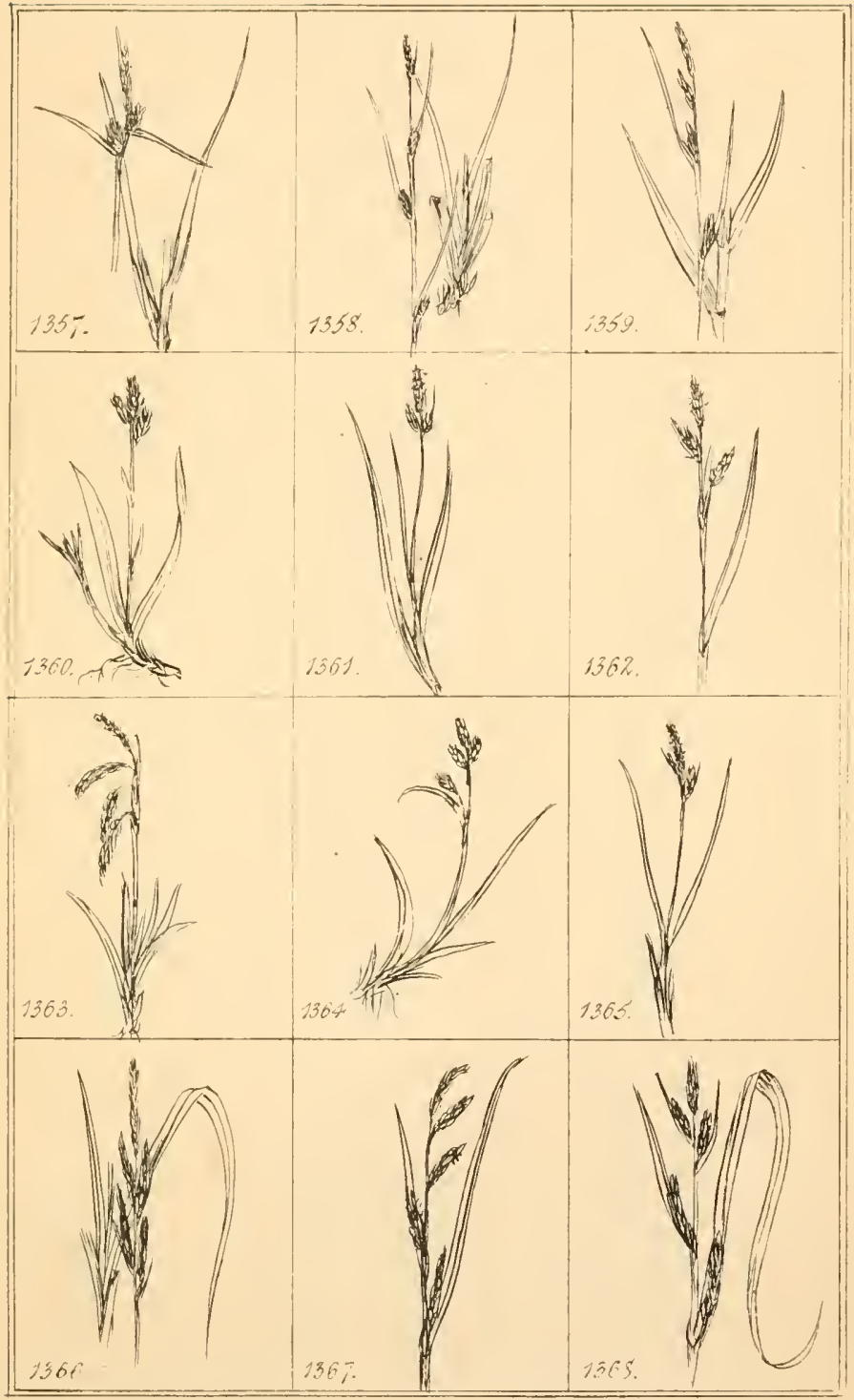



MOIJE EIA

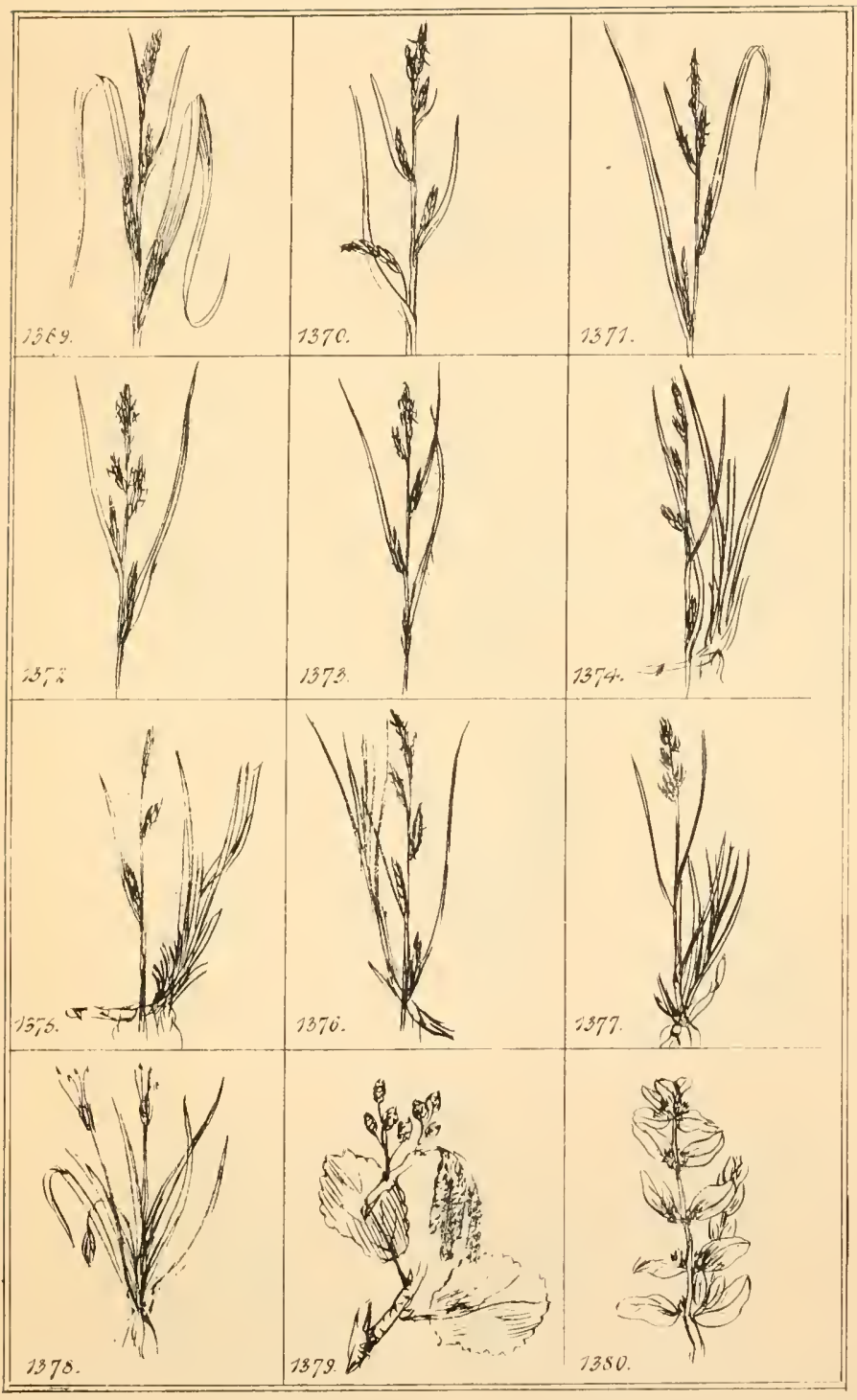



MUNECEIA

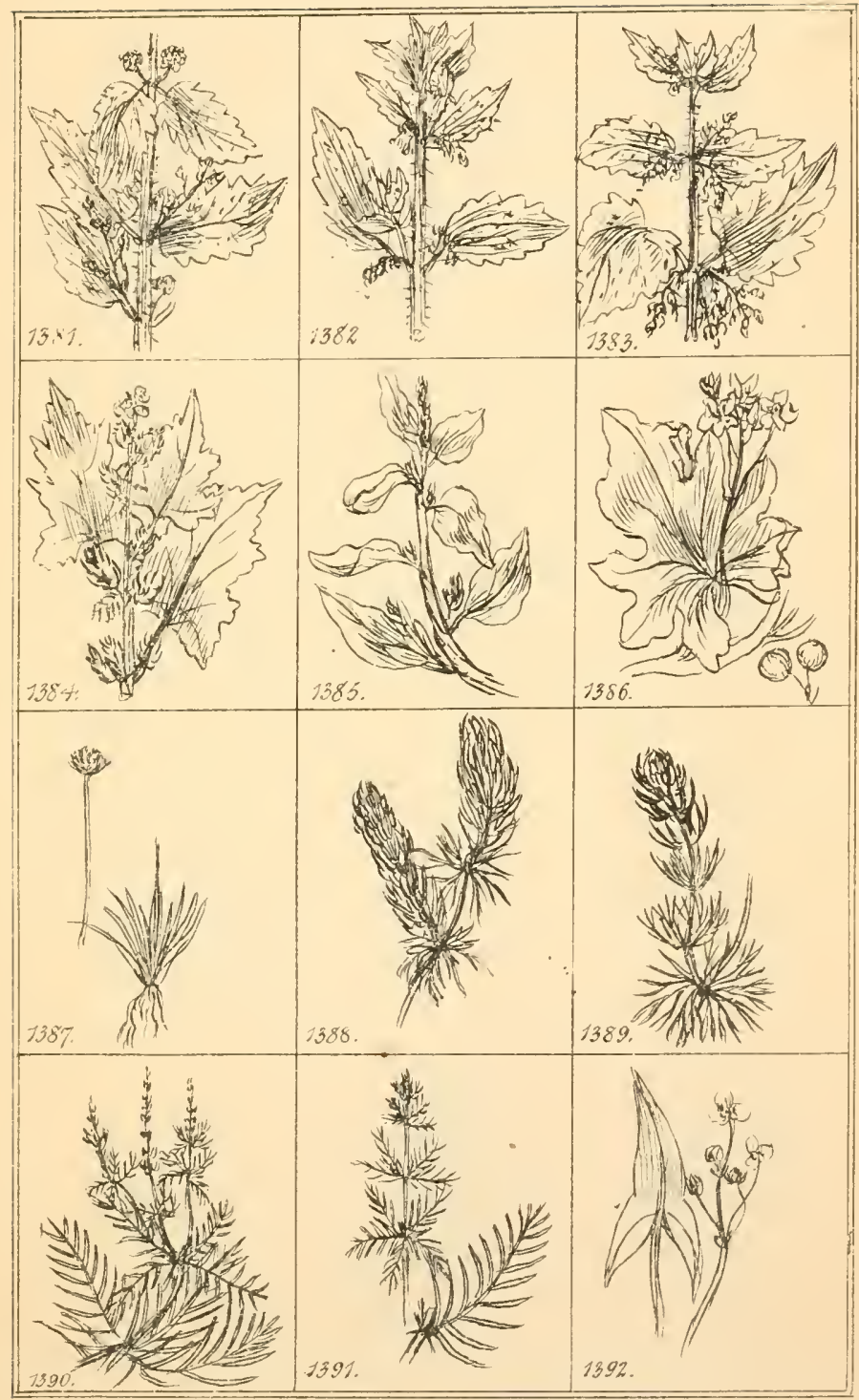



1) 10 E $\perp \mathrm{A}$

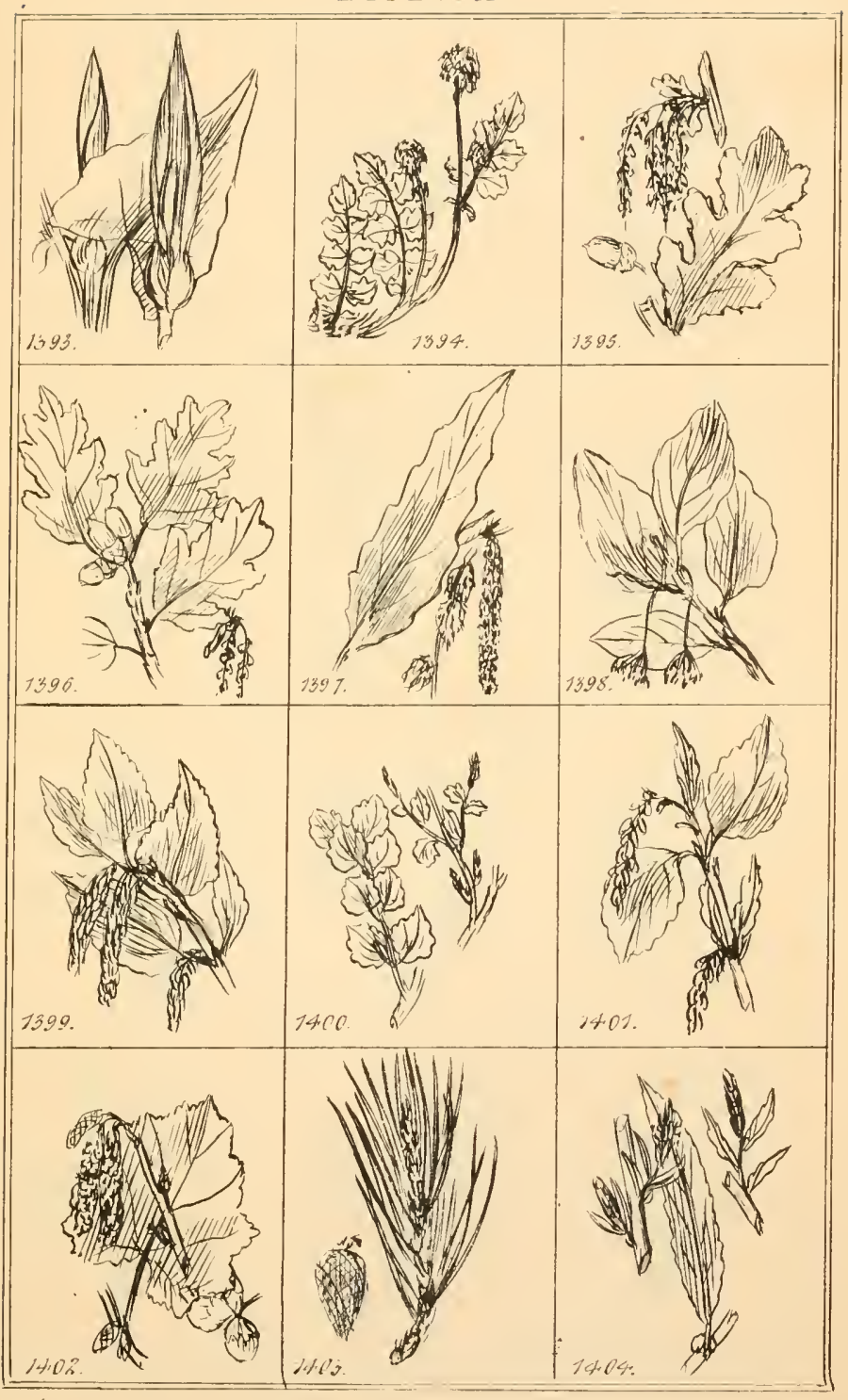



D IOECI is

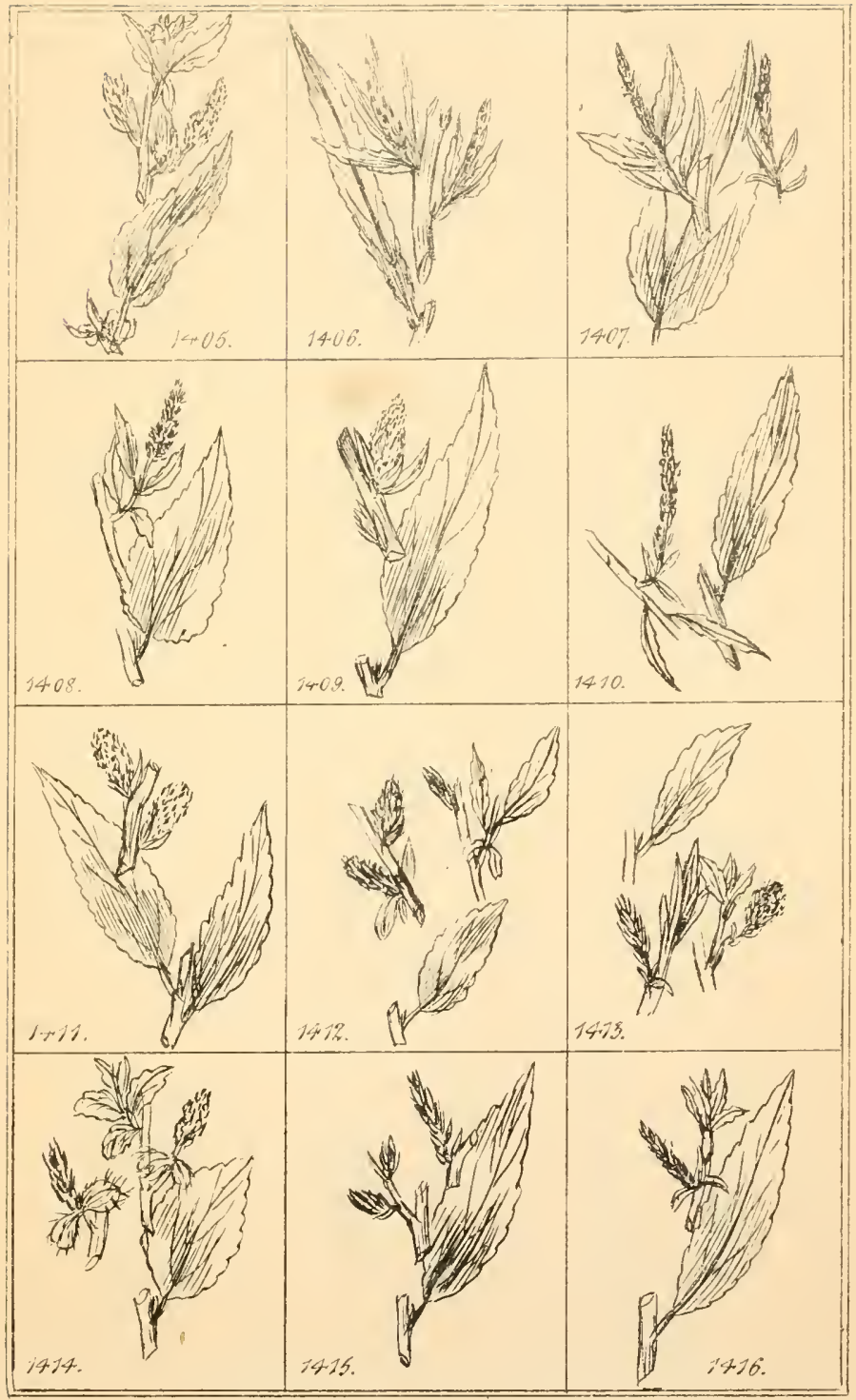



D IOEC LA

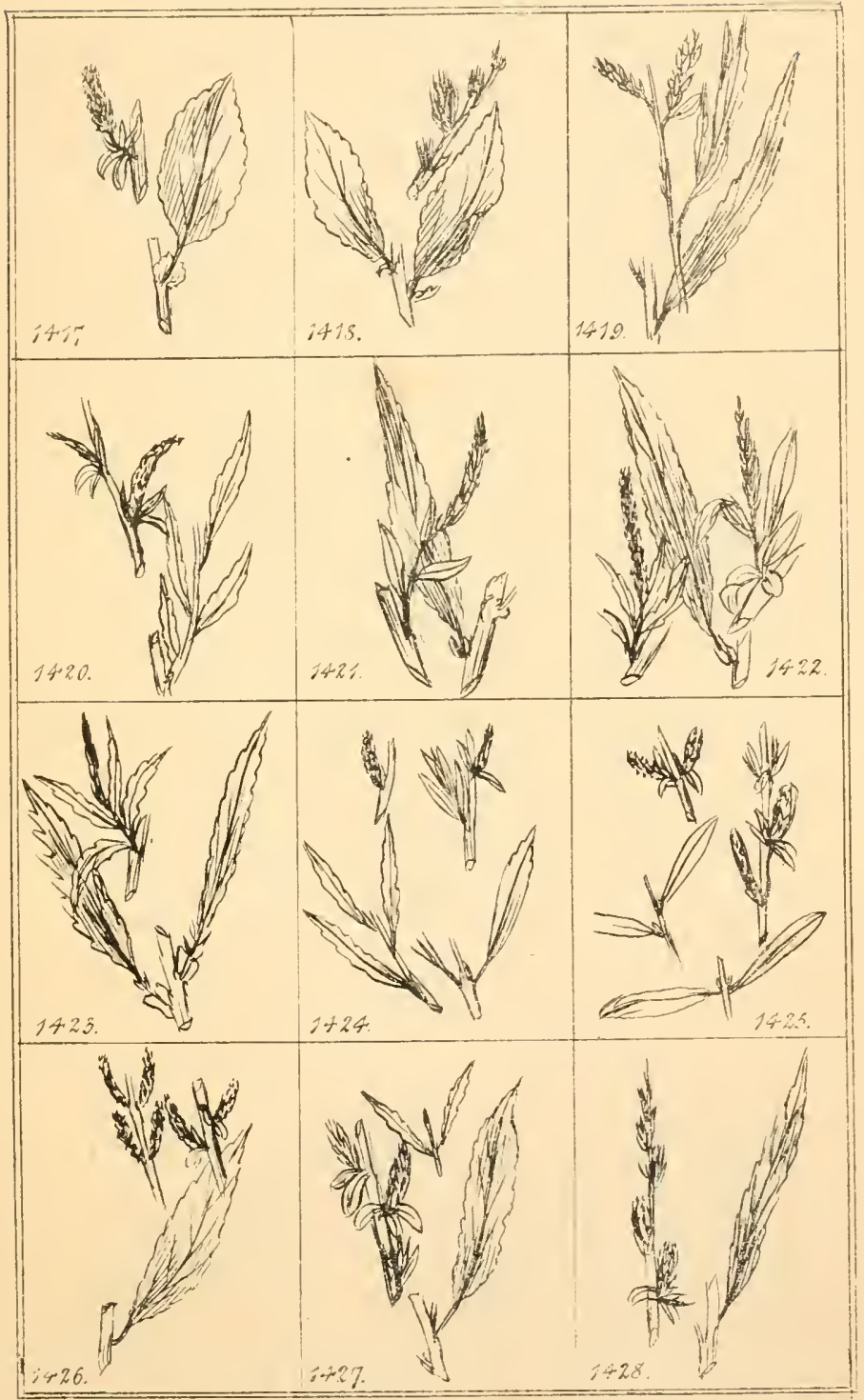



DIOEC $\perp \mathrm{A}$

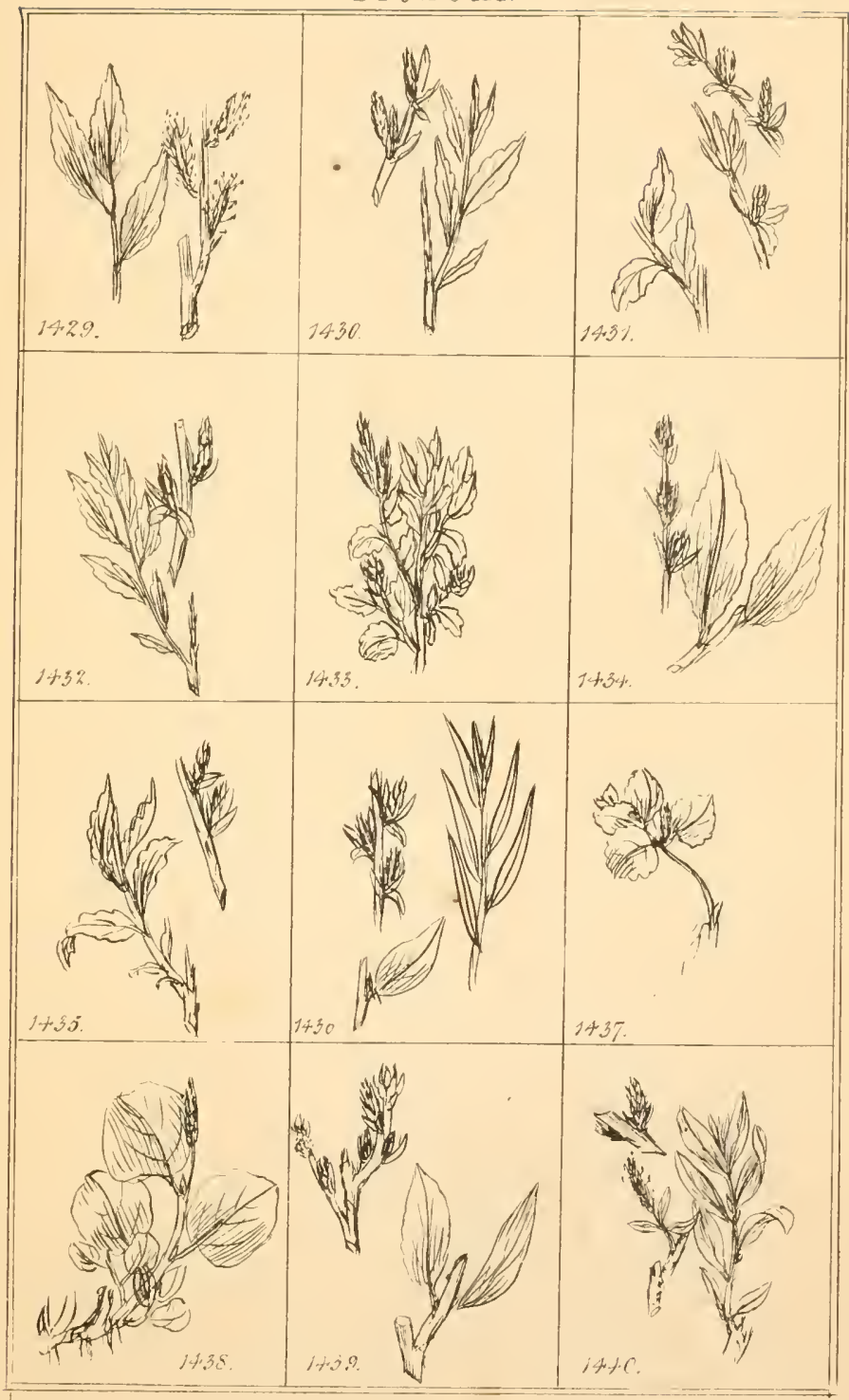





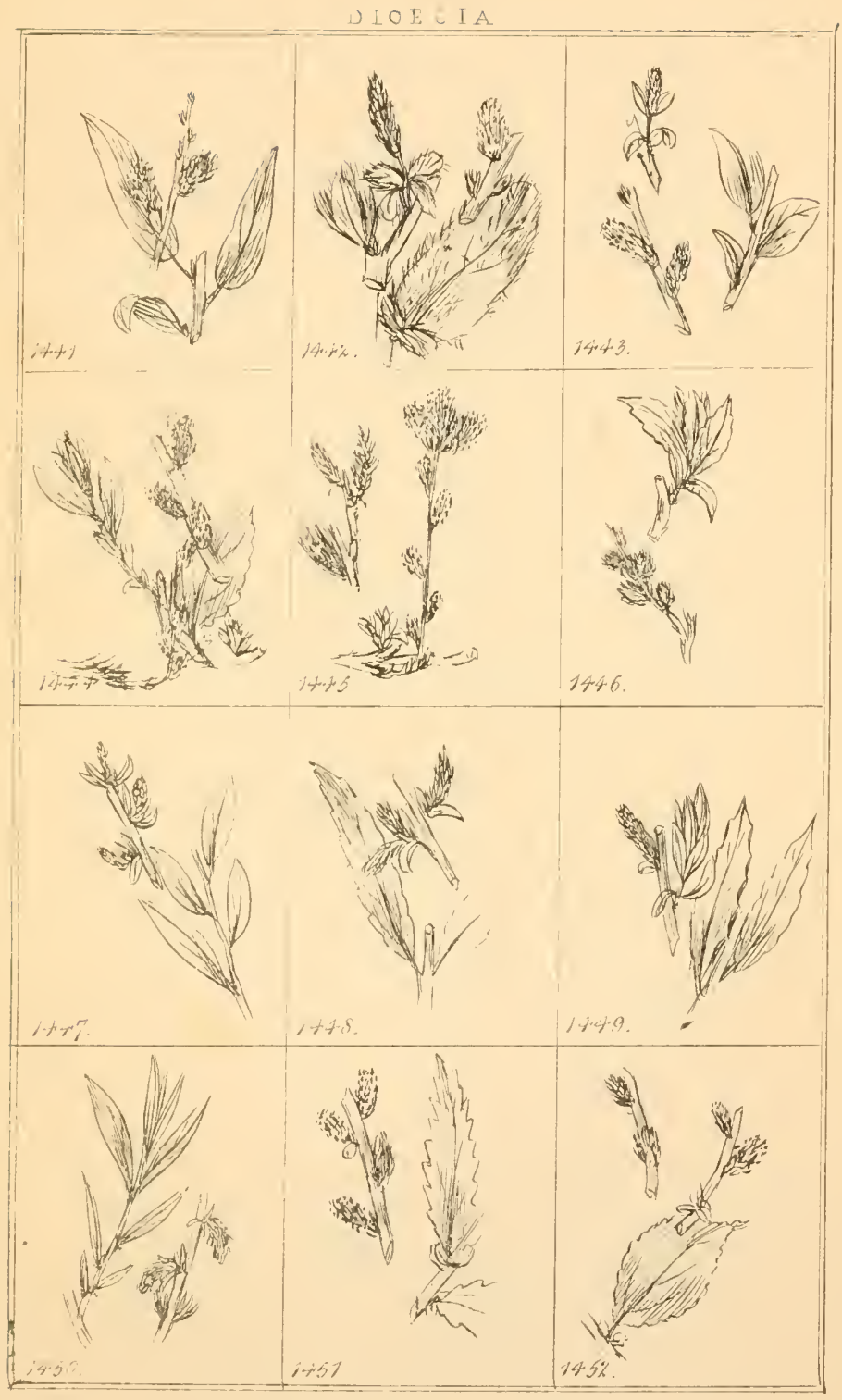



D IOECI A

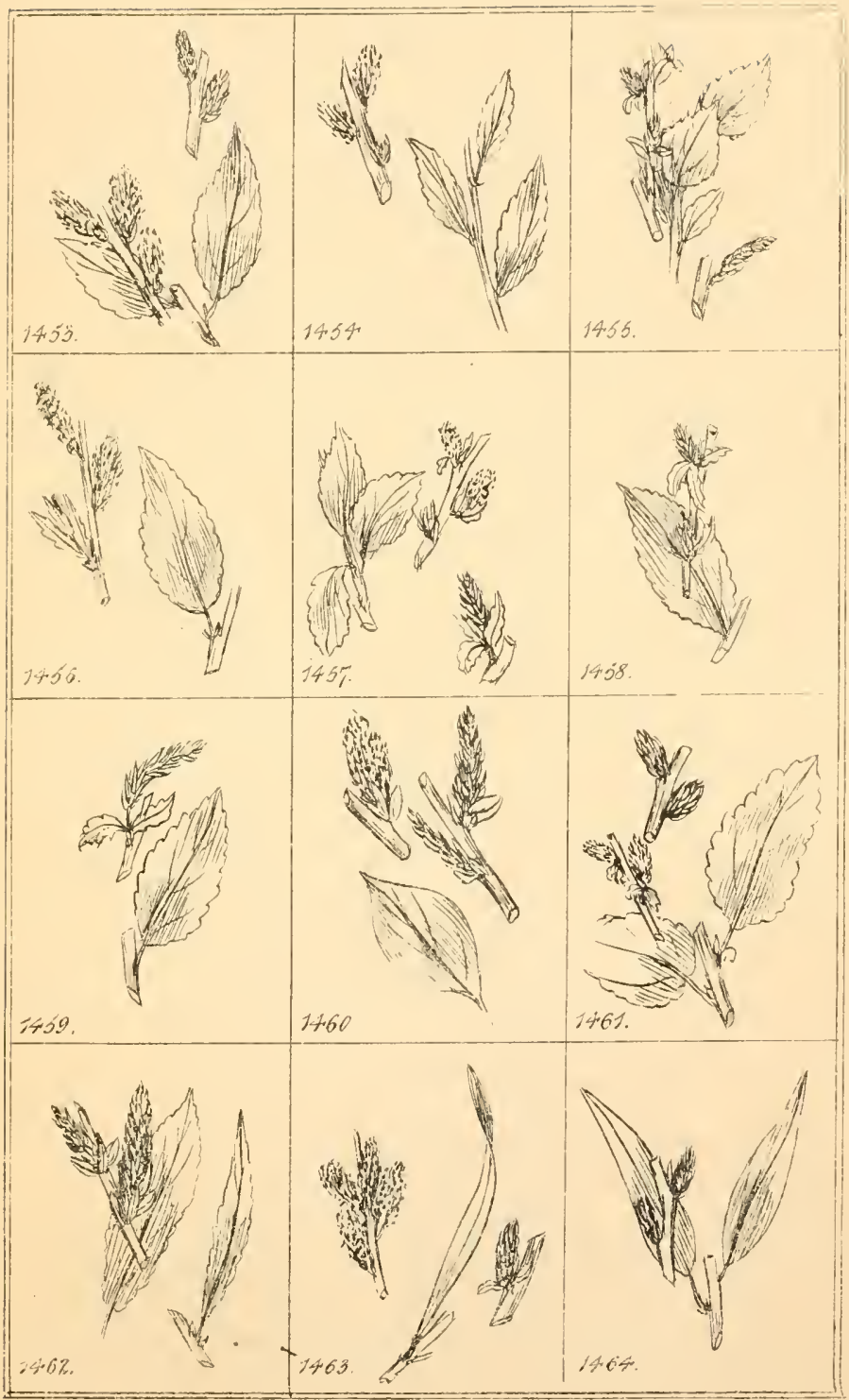



TIDEI'

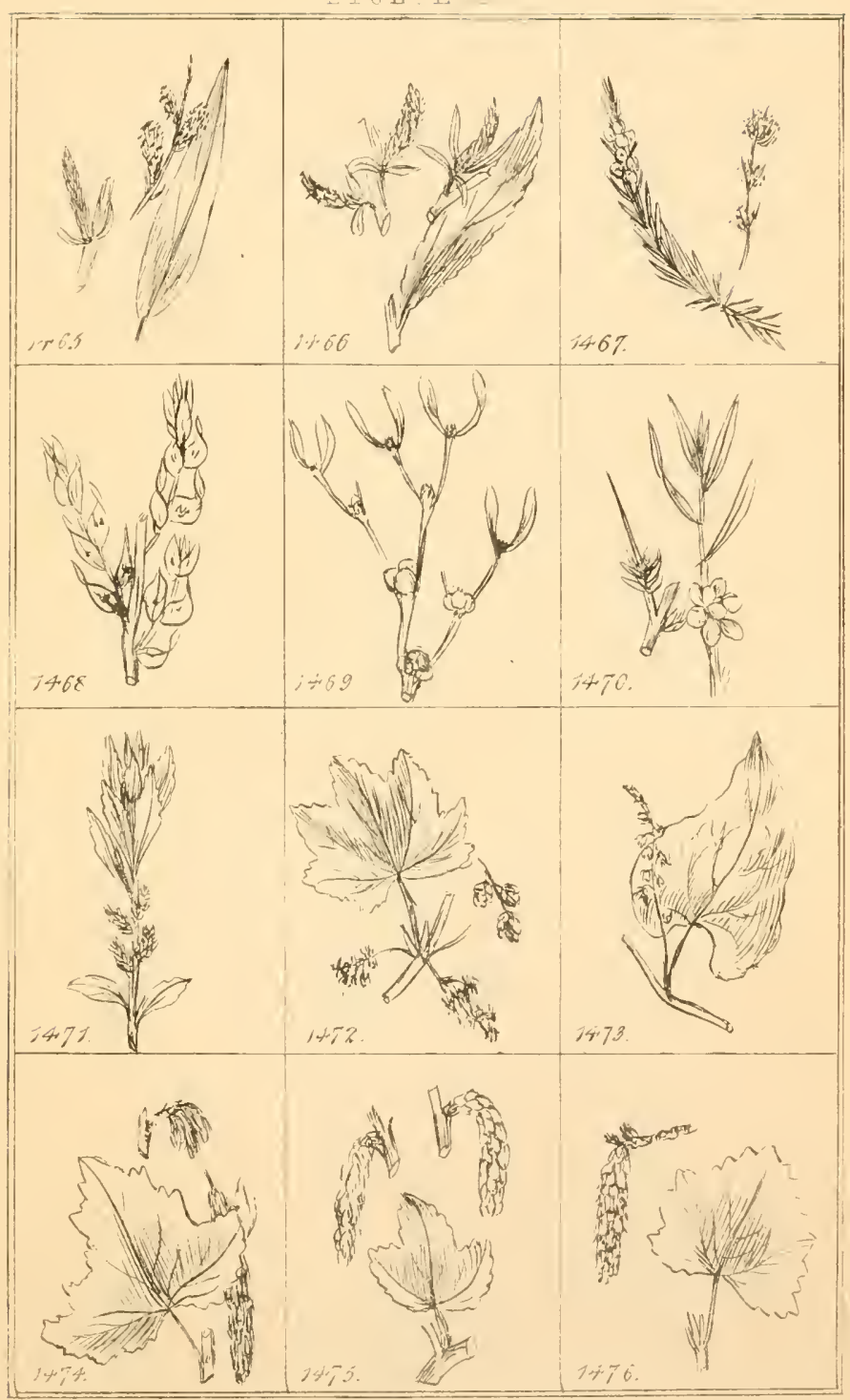





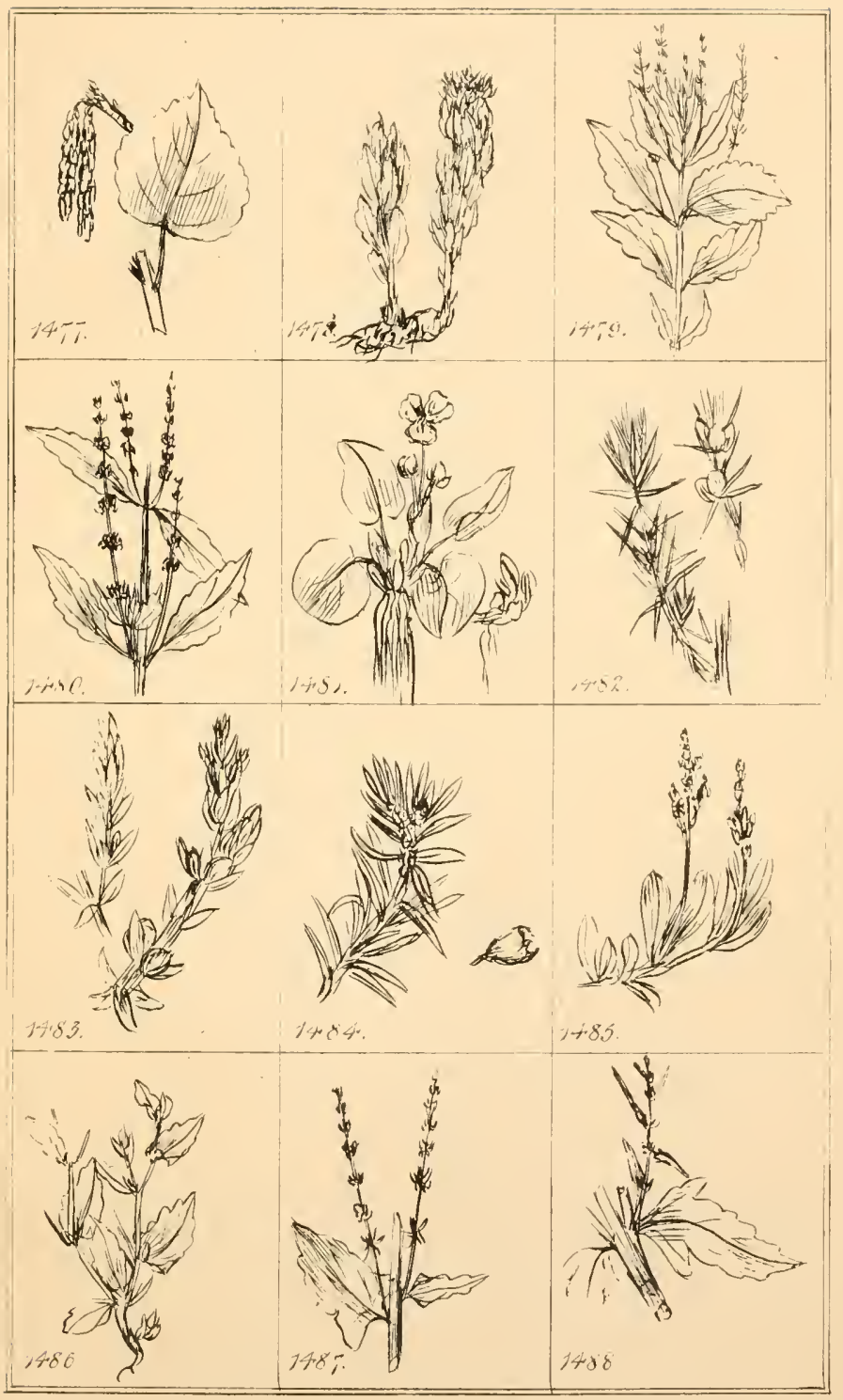



F $O Y \in A M I A$

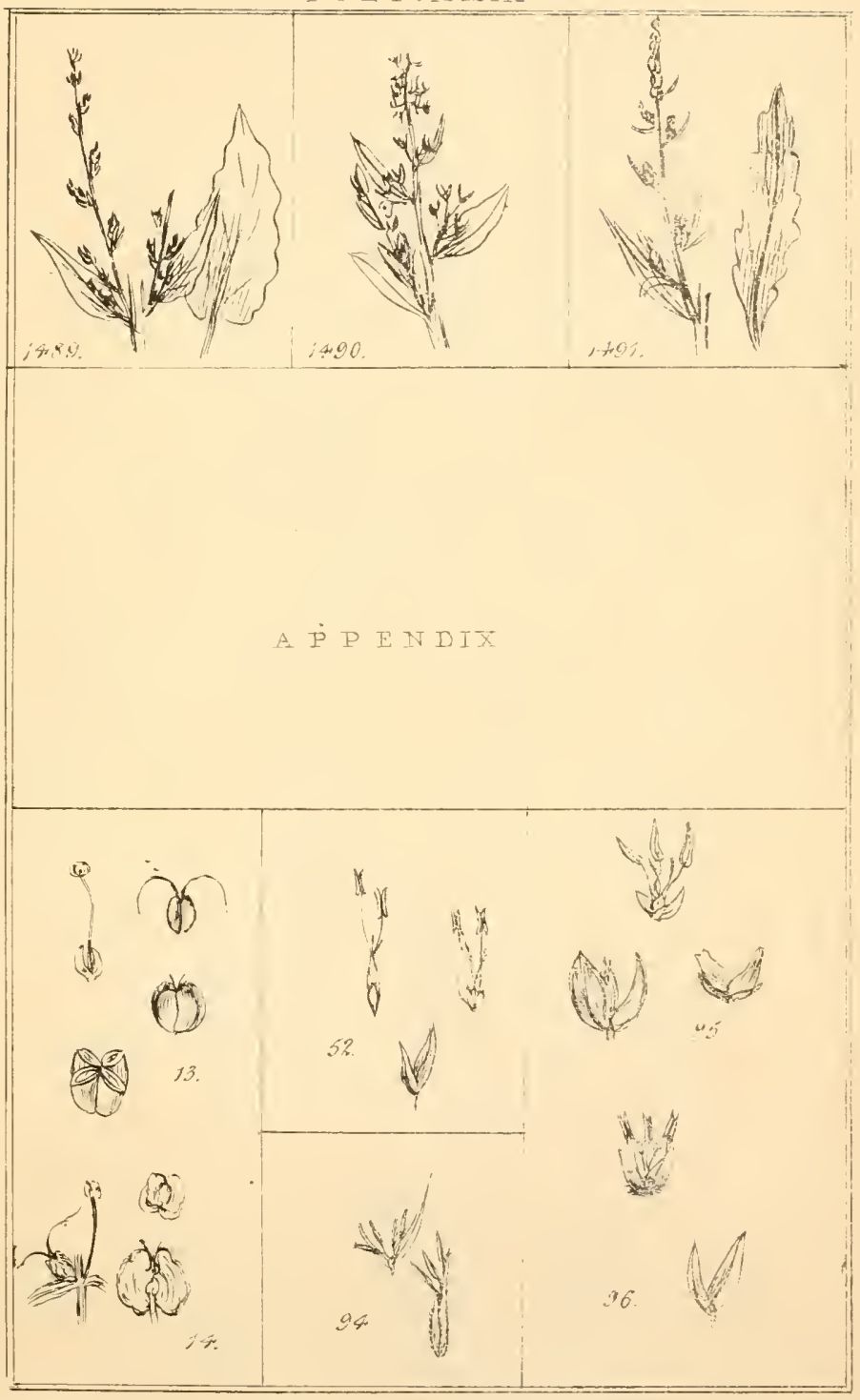



$A P P E N D I X$.

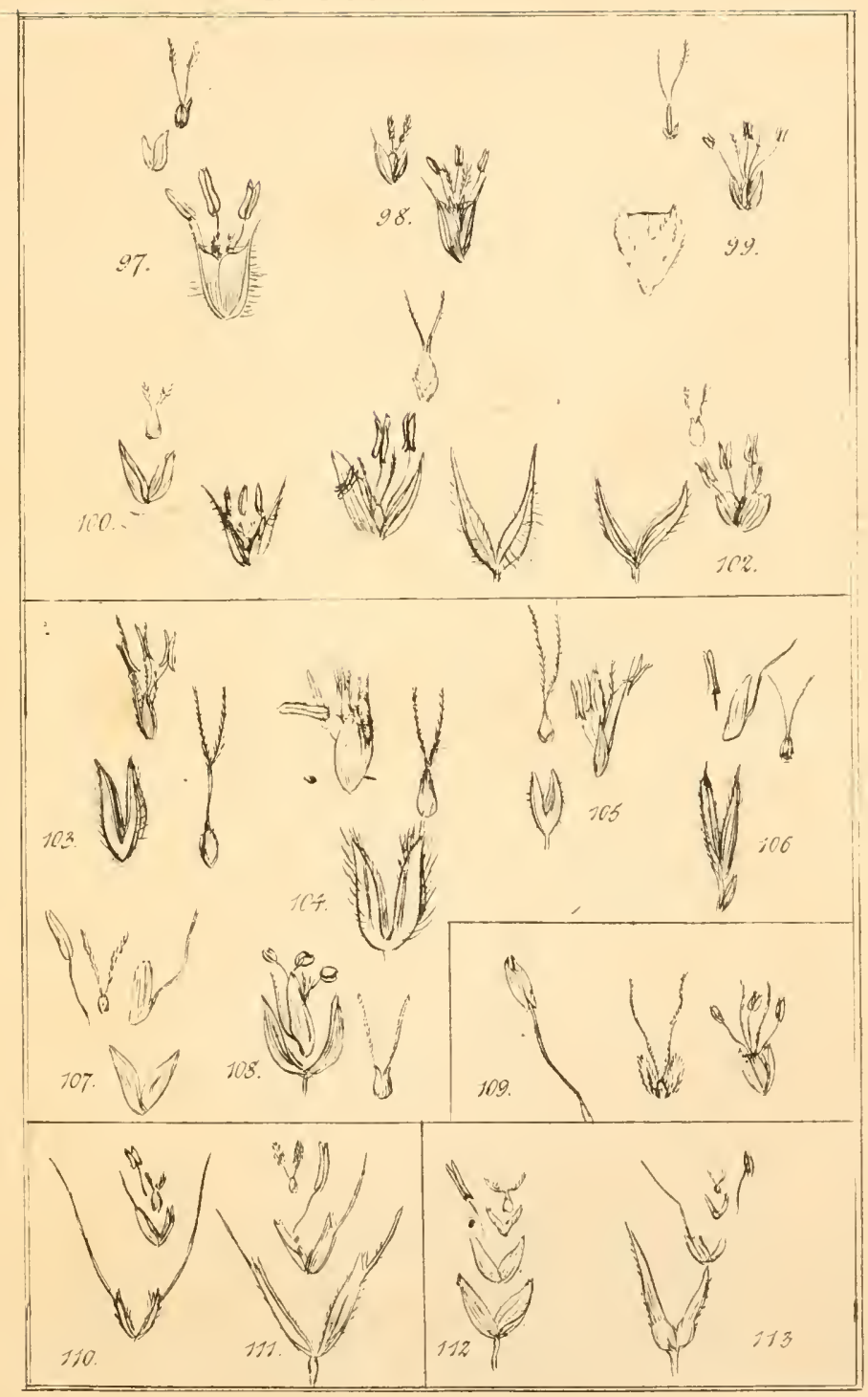



A P I I I DIX

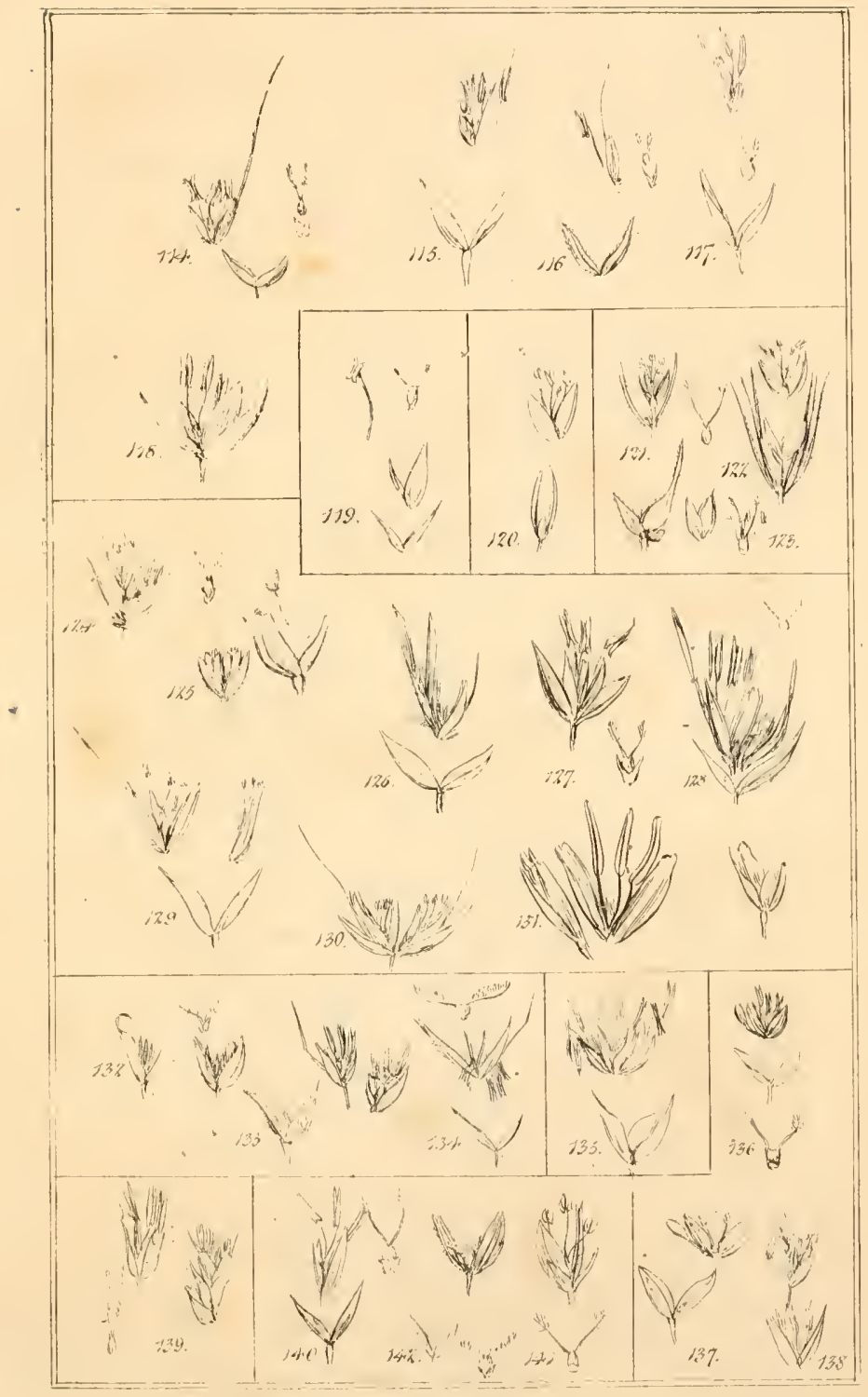



A P P E N D I X

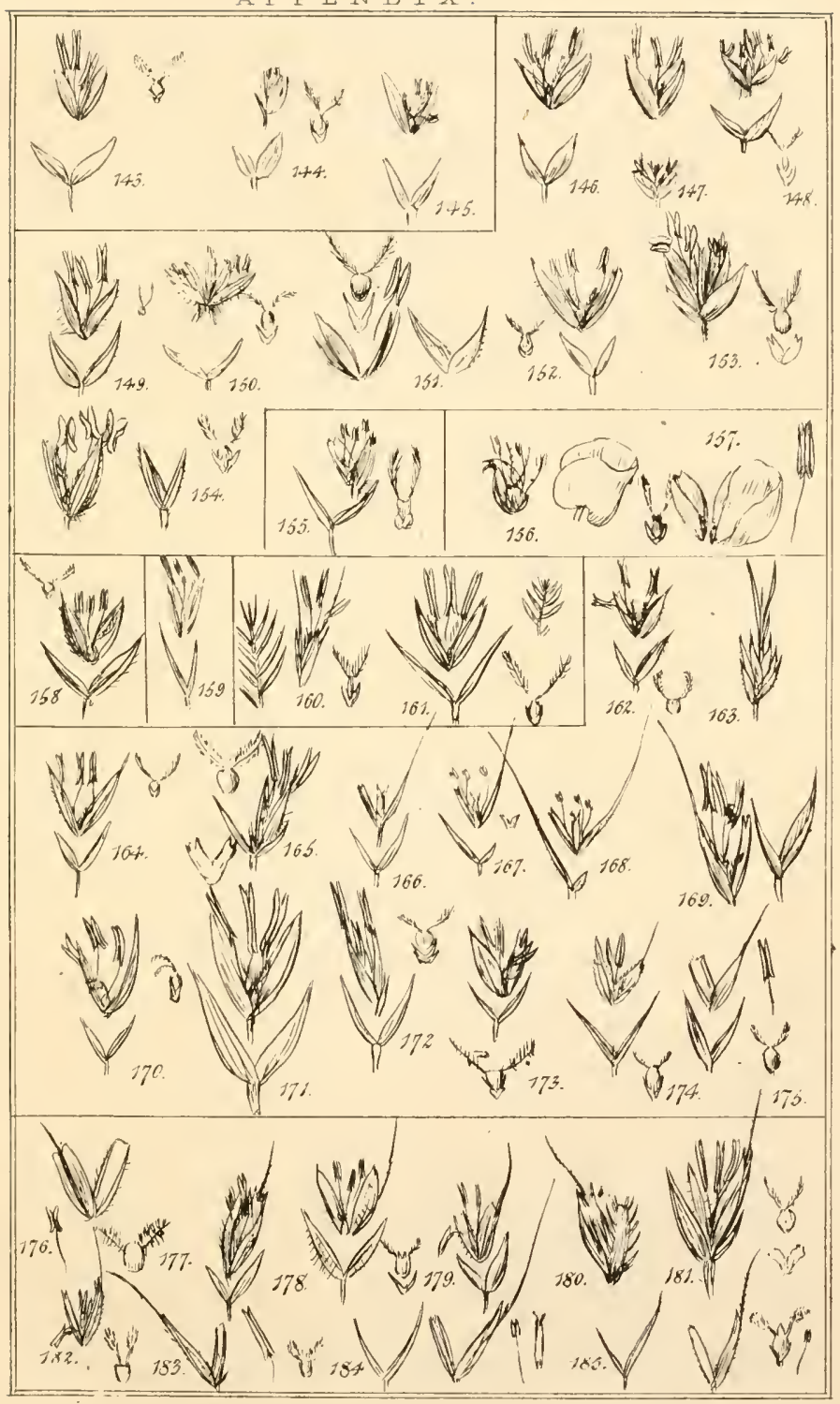



APPENDIX.

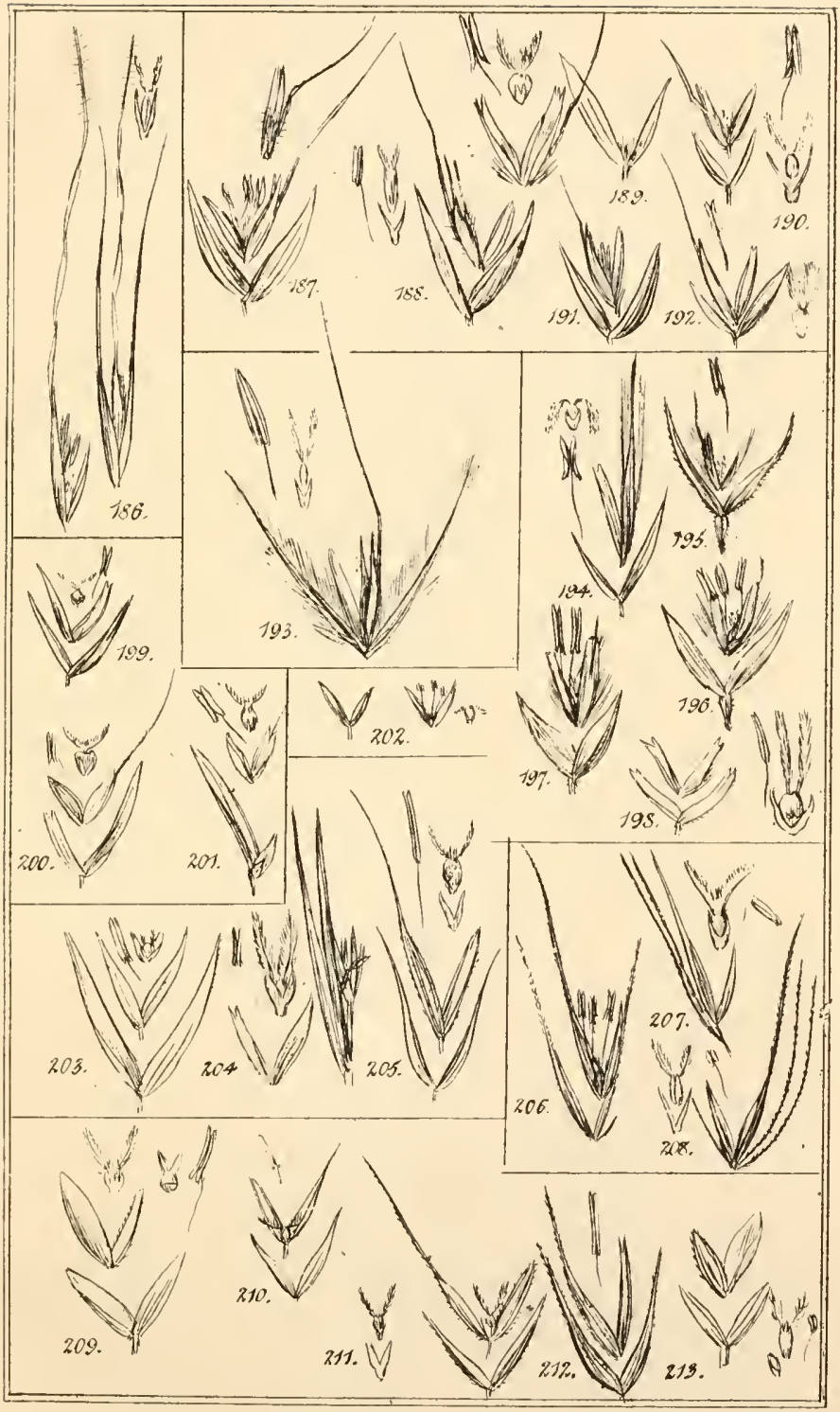





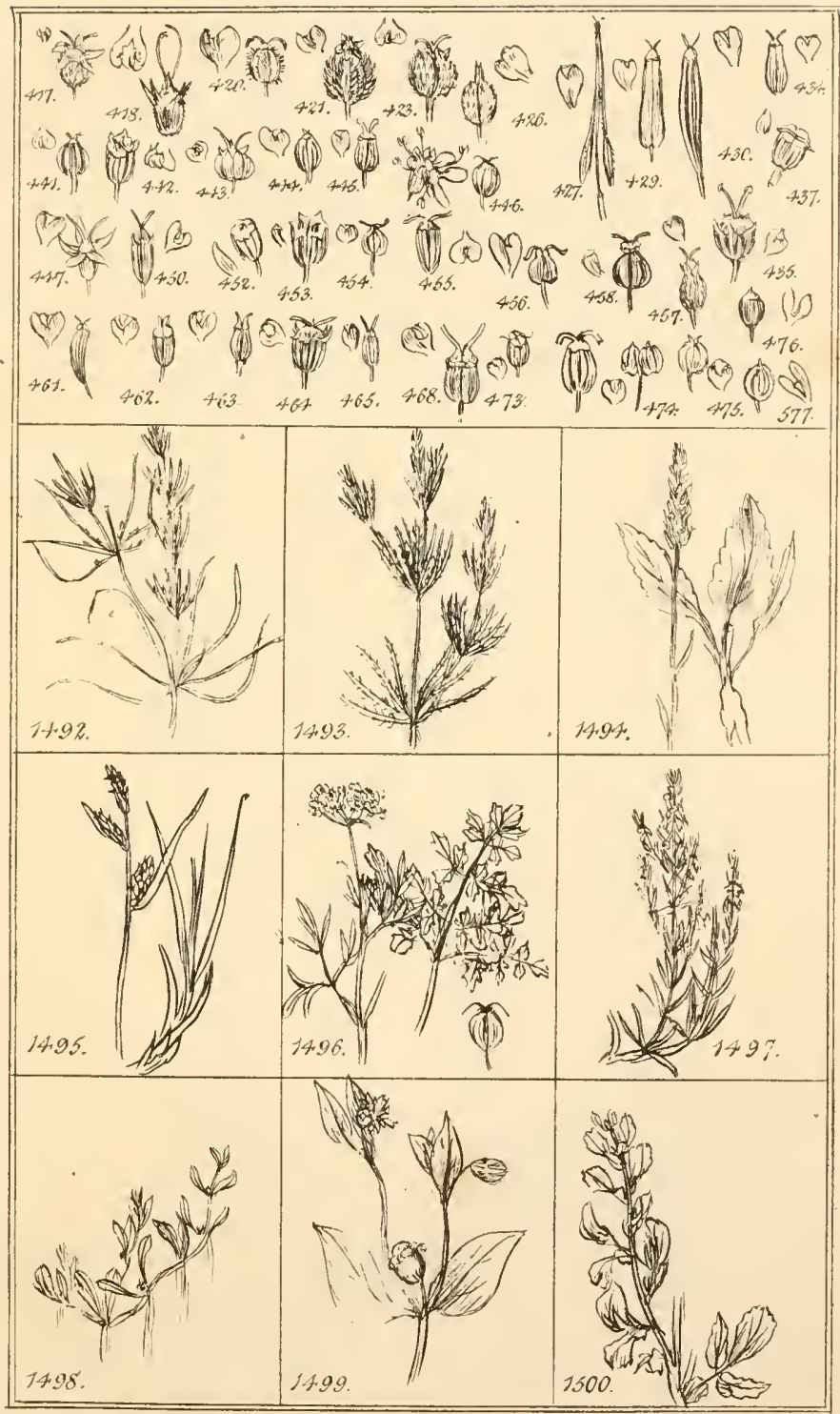



A P P E N I X

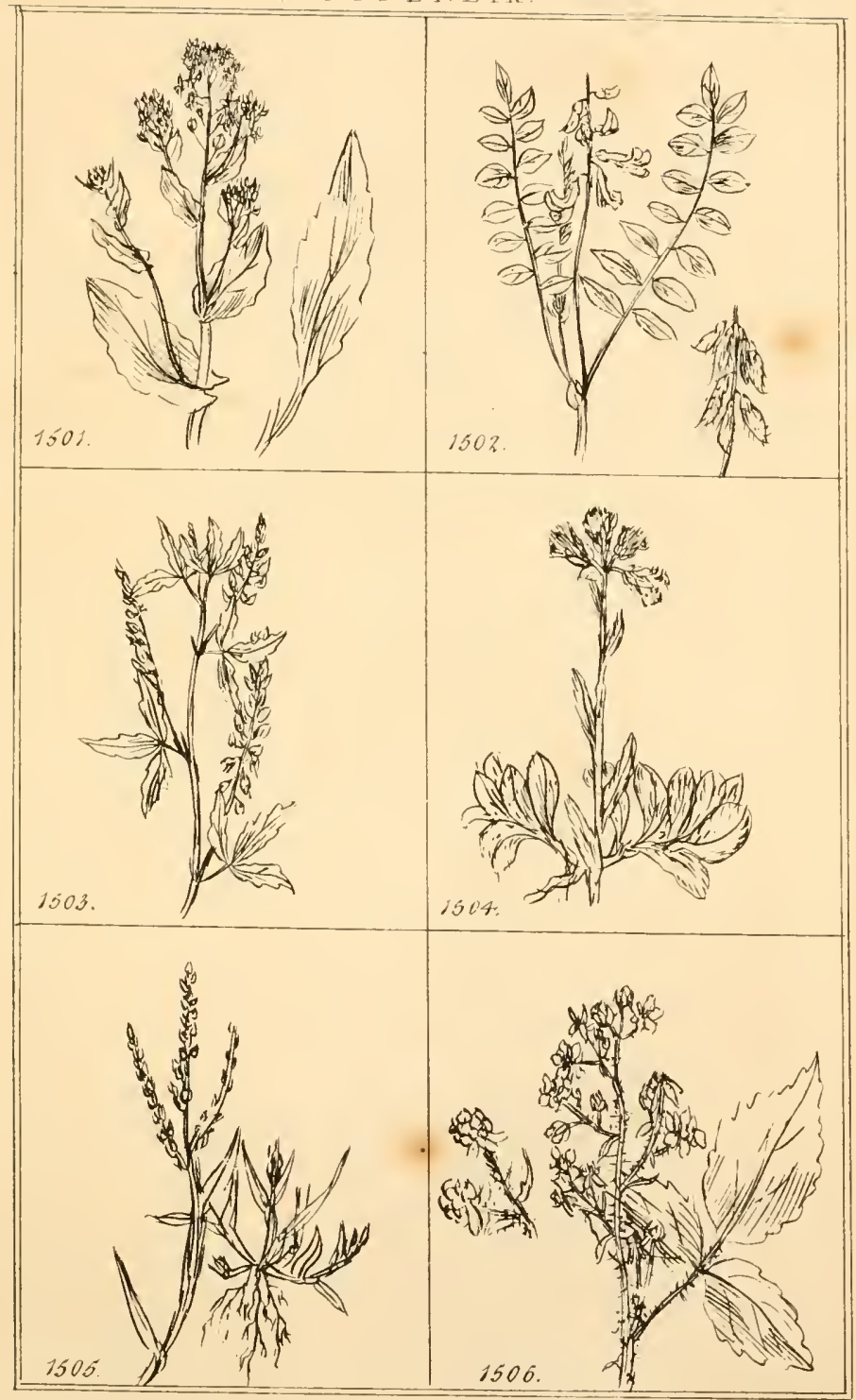





\section{ALPHABETICAL LATIN \\ I N D E X}

TO

\section{THE GENERIC AND SPECIFIC NAMES,}

\section{THE SYNONYMS}

OF SIR J, E, SMJTH'S ENGLISH FLORA-HOOKER'S BRITISH FLORA-

LINDLEY'S SYNOPSIS-AND WITHERING'S BOTANY.

Acer.

campestre, 610 .

pseudoplatanus, 609 .

Aceras.

anthropophora, 1274 .

Achillea.

Millefolium, 1251.

Ptarmica, 1249.

serrata, 1250.

tomentosa, 1252.

Achnodon L. arenarius L. 102 .

Acinos H.

vulgaris H. 905.

Aconitum.

napellus, 827 .

Acorus.

calamus, 529.

Actea.

$$
\text { spicata, } 501 \text {. }
$$

Adonis.

astivalis W. 839 .

autumnalis, 839 .

Adoxa.

Moschatellina, 622.

Agopodium.

Podograria, 455.

Æthusa.

Cynapium, 444.

Agrimonia.

Eupatorium, 729.

Agropyrum L.

canium L. 210.

cristatum L. 212.

junceum L. 209.

repens L. 210.

Agrostemma.
Githago, 709.

Agrostis.

alba, 118.

alpina W. 116.

eanina, 115.

littoralis W. 111.

palustris W. 118 .

setacea, 116.

spica venti, 114.

Aira.

vulgaris, 117.

alpina, 126.

aquatica, 133.

caspitosa, 125.

canescens, 128.

caryophyllea, 130 .

cristata, 124.

flexuosa, 127.

præcox, 129.

Airochloa $\mathbf{L}$.

cristata L. 124.

Ajuga.

alpina, 861.

chamæepitys, 863 .

Genevensis W. 861.

pyramidalis, 862 .

reptans, 860 .

Alehemilla.

alpina, 258.

arvensis, 259 .

vulgaris, 257 .

Alisma.

natans, 581

plantago, 579.

Damasonium, 580.

ranunculoides, 582 .

repens, 583 . 
Allium.

ampeloprasum, 504. arenarium, 505 . carinatum, 506. oleraceum, 507. schœnoprasum, 510. ursinum, 509, vineale, 508.

Aluus.

glutinosa, 1379 .

Alopecurus.

agrestis, 105.

alpinus, 104.

bulbosus, 106 .

fulvus, 108 .

geniculatus, 107. pratensis, 103.

Althæa. officinalis, 1035.

Alyssum. maritimum, 955.

Amaranthus.

blitum, 1385.

Amophila L. \& H. arenaria L. \& H. 198. arundinacea H. 198.

Anacamptis L. pyramidalis L. 1261 .

Anagallis.

arvensis, 318.

cærulea, 319. tenella, 320 .

Anchusa. officinalis, 293. sempervirens, 294.

Andromeda. polifolia, 626 .

Daboecia W. 626.

Androsæmum L. officinalis L. 1114.

Anemagrostis L. spica venti L. 114.

Anemone.

apennina, 832.

nemorosa, 831 .

pulsatilla, 830 .

ramunculoides, 833 .

Angelica.

archangelica, 457.

sylvestris, 458 .

Antemaria $L$.

dioica L. 1203.

Hyperborea, 1504 .

Anthemis.

arvensis, 1246.

cotula, 1247.

maritima, 1244.

nobilis, 1245 .

tinct oria, 1248.

Anthericum.

serotinum, 522.

Anthoxanthum.

odoratum, 52.
Anthriscus.

cerefolium H. \&L, 428

sylvestris H. \& L.429.

vulgaris, 426 .

Anthyllis. vulneraria, 1054.

Antirrhinum.

arvense W. 933

Cymbalaria, 926.

elatine, 928.

linaria, 930 .

majnus, 932.

minus, 931.

Orontium, 933.

repens, 929.

spurium, 927.

Apargia.

autumnalis, 1141.

hirta, 1139.

hispida, 1138.

Taraxaci, 1140.

Apium.

graveolens, 554. petroselinum, 1496.

Aquilegia. vulgaris, 828 .

Arabis.

ciliata, 1003.

hirsuta, 1004.

hispida, 1002.

petræa L. \& H. 1002.

thaliana, 1000 .

turrita, 1005.

stricta, 1001.

Arbutus.

alpina, 628 .

unedo, 627 .

uva-ursi, 629.

Archangelica L.

officinalis L. 457 .

Arctium.

Bardana, 1172.

lappa, 1171.

Arctostaphylos L.

1) alpina L. 628 .

1) uva-ursi L. 629.

Arenaria.

ciliata, 690.

fasciculata L. 689.

fastigiata, 689 .

hirta, 688 .

marina, 692 .

media, 892 .

norvegia $\mathrm{H}$.

peploides, 683.

rubella, 688 .

rubra, $69 \mathrm{I}$.

tenuifolia, 686 .

trinervis, 684 .

serpyllifolia, 685 .

verna, 687.

Aristolochia.

Clematitis, 1297. 
Armeria L.

maritima L. 487.

Arrhenatherum H. \& L. avenaceum H.\& L. 134

Artemesia

Absinthium, 1198.

campestris, 1195 .

cærulescens, 1200 .

Gallica, I197.

maritima, 1196.

vulgaris, 1199 .

Arum.

maculatum, 1393.

Arundo.

arenaria, 198.

calamagrostis, 196.

epigejos, 195.

Phragmites, 194. stricta, 197.

Asarum.

europæum, 726.

Asparagus.

officinalis, 524.

Asperugo.

procumbens, 302.

Asperula.

cynanchica, 225.

odorata, 224.

Aster.

Tripolium, 1228.

At hamanta.

libanotis, 464 .

Astragalus.

alpinus H. 1502 .

campestris, 1084.

glycyphyllos, $108 \mathrm{I}$.

hypoglottis, 1082 .

uralensis, 1083.

Atriplex.

angustifolium, 1488 .

erecta, 1489 .

laciniata, 1486 .

littoralis, 1490. $14 \mathrm{q} /$

marina L. \& W. 1490

patula, 1487 .

pedunculata, 1491. I L GO

portulacoides, 1485.

Atropa.

Belladonna, 357.

Arena.

alpina, 191.

elatior W. 134.

fatua, 187.

flavescens, 192.

pratensis, 190.

pubescens, 189.

Azalea. strigosa, 188.

procumbens, 321 .

Ballota.

nigra, 899.

Barbarea.

præcox, 992 . vulgaris, 991.

Bartsia.

alpina, 913.

Odontites, 915.

viscosa, 914.

Bellis.

perennis, 1237.

Berberis.

Beta. vulgaris, 559 .

maritima, 398.

Betonica. officinalis, 893.

Betula.

alba, 1399.

nana, 1400.

Bidens.

cernua, 1190.

tripartita, 1189.

Blysmus L. \& H. compressus L. H. 78. rufus H. 79.

Borago.

officinalis, 301

Borkhafisia H. fotida, 1161 .

Brachypodium H. \& L. pinnatum H. L. 175 . sylvatieum H. L. I74.

Brassica.

campestris, 1009 .

monensis, 1011.

napus, 1007.

oleracea, $10 \mathrm{I0}$.

Briza. Rapa, 1008.

media, 157. minor, 156.

Bromus.

arvensis, 181.

asper, 183.

diandrus, 185.

erectus, 182.

giganteus H. 169.

mollis, 178.

pinnatus $\mathrm{W}, 174$.

racemosus, 179 .

secalinus, 176 .

squarrosus, 180.

sterilis, 184.

sylvaticus W. 174 .

velutinus, 177 .

Bryonia.

dioica, 1386.

Buffonia.

tenuifolia, 266.

annua L. \& H. 266.

Bunium.

flexuosum, 434.

Bupleurum.

Odontites, 470.

rotundifolium, 469.

telluissimum, 471 . 
Butomus.

umbellatus, 624 .

Buxus. sempervirens, 1380 .

Cakile. maritima, 976.

Calamagrostis W. H. \& L. Epigejos W. H. \& L. 195. lanceolata W.'H. \& L. 136. stricta W. H. \& L. 197.

Calamintha $\mathrm{H}$. nepeta H. 907. officinalis H. 906.

Callitriche. autumnalis, 14 . pedunculata, H. 14. verna, 13.

Calluna. vulgaris, 602.

Caltha. palustris, 858 . radicans, 859 .

Camelina. sativa, 956 .

Campanula. glomerata, 333. hederacea, 335. hybrida, 334 . latifolia, 330 . jatula, 327 . persicifolia, 329. ranunculoides, 331 .

Rapunculus, 228. roturidifolia, 326 . Trachelium, 332 .

Eaprifolium L. perfoliatum L. 265. periclynenum L. 366 .

Capsella L. \& H. Bursa-pastoris, L. H. 966.

Cardamine.

amara, 983.

bellidifolia, 979

bulbifera W. 978 .

flexuosa W.981.

hirsuta, 981.

impatiens, 980. pratensis, 982 .

Carduus. acanthoirles, 1177. acaulis W. 1186. arvensis W. 1181. eriophorus W. 1182. heterophyllus W.1184. lanceolatus WV. 1179. marianus, 1178 . nutans, 1175 . pratensis W. 1185 palustris IV. 1180. tenuiflorus, 1177 . Carex. acuta, 1367.

ampullacea, 1372 . anyustifolia, 1375 . arenaria, 1329. atrata, 1351. atro-fusea L. 1350. axillaris, 1327. binervis, 1359 . cæspitosa, 1365 canescens $H$. capillaris, 1346. clandestina, 1338. eurta, 1323.

Davalliana, 1319. depauperata, 1342. digita, 1337. dioica, 1318. distans, 1358. divisa, 1331 . devulsa, 1333. eclinuata L. 1312. elongata; 1324 . extensa, 1357. filifurmis, 1376 . flava, 1354. fulva, 1356 . hirta, 1373. incurva, 1328 intermedia, 1330 . laevigata, 1370 . leporina $\mathrm{H}$. limosa, 1349. Miclichoferi, 1343. muricata, 1332 . œederi, 1355. ovalis, 1325 . pallescens, 1353. paludosa, 1368. panicea, 1362 . paniculata, 1336. pauciflora, 1321 . pendula, 1339. phæostachya, 1345 . pilulifera, 1361 . præcox, 1360. pseudo-cyperus, 1348. pulicaris, 1320 . pulla, 1352. punctata $\mathbf{H}$. rariflora, 1347. recurva, 1363. remota, 1326. rigida, 1364 . riparia, 1369. rufus W. 79 .

saxatalis H. 1352 . speirostachya, 1344. stellulata, 1322 . stictocarpa, 1374. stricta, 1366. strigosa, 1340. sylvatica, 1341 . 
teretiuscula, 1335.

tomentosa L. 1362.

ustulata, 1350.

vesicaria, 1371.

Carlina. vulpina, 1334 .

\section{Carpinus.} vulgaris, 1188.

Carum.

$$
\text { Betulus, } 1401 .
$$

carui, 463. verticillatum L. 440.

\section{Castanea.}

vesca W. \& L. 1397 vulgaris W. \& H. 1397.

Catabrosa H. \& L. 131. aquatica H. \& L. 131.

Catopodium L. loliacea L. 213.

Caucalis. daucoides, 421. latifolia, 42:2.

\section{Centaurea.}

ealcitrapa, 1258.

cyanus, I255

jacea, 1253

Isnardi, 1257.

nigra, 1254.

Scabiosa, 1256. solstitialis, 1259.

Centranthus L. latifolia L. 53.

Centunculus. minimus, 250.

Cerastium.

alpinum, 719. aquaticum, 721 . arvense, 718 . latifolium, 720 . semiclecandrum, '7 76 . tetrandrum, 7 I7. viscosum, 715 . vulgatum, 714 .

Cerasus L.

avium L. 734.

Padus L. 733.

Ceratophyllum. demersum, 1388. submersum, 1399.

Chærophyllum.

aromaticum, H. \& L. 433. aureum, H. \& L. 432 .

sativum, 428.

sylvestre, 429 .

temulentum, H. \& L. 431.

Chamagrostis, L. minima, L. 109.

Chara.

asper, 1493.

flexilis, 9 .

gracilis, 12 .

heduraii, 1492 .

hispida, 8 . nidifica, 11 .

translucens, 10.

vulgaris, 7 .

Cheiranthus.

cheiri, IV. H. \& L. 996.

fruticulosus, 996.

incanus, WV. 997.

sinuatus, W. 998.

Chelidonium.

majus, 802.

Chenopodium.

acutifolium, 396.

album, 391.

bonus Henricus, 385.

botryodes, 388 .

ficifolium, 392.

fruticosum, H. \& L. 400.

glaucum, 393.

hybridum, 390 .

intermedium, H. 386 .

maritimum, 397.

murale, 389.

olidum, 394.

polysperinum, 395.

rubrum, 387

urbicum, 386 .

Cherleria.

sedoides, 693.

Chlora.

perfoliata, 595.

Chondrilla, L. muralis L. 1135.

Chrysanthemum. leucanthemum, 1238 segetum, 1239 .

Chrysocoma. linosyris, 1192.

Chrysosplenium. alternifolium, 635. oppositifolium, 636.

Cichorium. intybus, 1170 .

Cicuta. virosa, 443.

Cineraria. campestris, H. \& L. 1235. integrifolia, 1235. palustris, 1234 .

Circea.

alpina, 19.

lutetiana, 18.

Cistus.

guttatus, 819 .

belianthemum, 822 .

ledifolius, 820 .

marifolius, 818 .

polifolius, 824.

surrejanus, 821 .

tomentosus, 823 .

Cladium.

mariscus, 51.

Clematis.

vitalba, 834 . 
Clinopodium.

Cnicus. vulgare, 902.

acaulis, 1186.

arvensis, 1181 .

eriophorus, 1182.

heterophyllus, 1184 .

lanceolatus, 1179.

palustris, 1180.

pratensis, 1185.

tuberosus, 1183 .

Cnidium.

silaus, 468.

Cochlearia.

anglica, 969.

Armoracia, 971.

danica, 970 .

gronlandica, 968.

officinalis, 967 .

Colchicum.

autumnale, 578 .

Comarum.

palustre, 800 .

Condylocarpus $\mathbf{L}$. officinalis, L., 477.

Conium. maculatum, 445 .

Conopodium L: flexuosum, L., 434.

Convalaria.

majalis, 525 .

multiflora, 528 .

Polygonatum, 527.

verticillata, 526 .

Convolvulus.

arvensis, 322.

sepium, 323.

Conyza. soldanella, 324 .

squarrosa, 1211.

Coralorrhiza. innata, 1295.

Coriandrum. sativum, 446.

Cornus.

sanguinea, 253.

suecica, 254.

Coronopus, W. H. L.

didyma, W. H. L. 973. Ruellii, W. H. L. 972.

Corrigeola.

littoralis, 485.

Corydalis, H. L.

bulbosa, H. L. 1040. claviculata H. L. 1042 solida H. L. 1040.

Corylus. Avellana, 1402.

Corynephorus L. canescens L. 128.

Cotoneaster L. H. vulgaris L. H. 740.

Cotyledon. lutea, 695 .

umbilicus, 694 .

Crambe.

maritima, 977.

Cratægus W. H. L.

Crepis. oxyacantha W. H. L. 738.

biennis, 1164.

fotida, 1161 .

pulchra, 1162.

succicæfolia H. 1153.

tectorum, 1163.

virens H. 1163.

Crithmum.

maritimum, 452.

Crocus.

aureus H. 60 .

minimus H. 61 .

nudiflorus, 62.

reticulatus, 61 .

sativus, 59.

speciosus H. 62 .

vernus 60 .

Cucubalis.

baccifer, 1499.

Cuscuta.

epithymum, 407.

europaea, 406.

Cyclamen.

hederifolium, 310.

Cynorlon. dactylon, 119.

Cynglossum.

officinalis, 295.

sylvaticum, 296.

Cynosurus.

cristatus, 161 .

echinatus, 160.

Cyperus

fuscus, 70 .

longus, 69 .

Cypripedium. calceolus, 1296.

Cytisus H. L.

Dactylis. scoparius H. L. 1047.

glomerata, 158.

Dapline.

laureola, 608 .

Mezereum, 607.

Datura.

Stramonium, 355 .

Daucus.

carota, 419.

maritimus, 420 .

Delphinium. consolida, 826 .

Dentaria. bulbifera, 978.

Deschampsia L. crespitosa L. 125.

Dianthus. armeria, 660 . cæsius, 664 . 
caryophỵllus, 662 . deltoides, 663. prolifer W. H.66I

Digitalis. purpurea, 938 .

Digitaria. humifusa H. 1505. sanguinalis, 120. stolonifera L. 119.

Digraphis. arundinacea, 96 .

Diotis. maritima, 1193.

Diplotaxis $\mathbf{L}$. muralis L. 1016. tenuifolia L. 1015.

Dipsacus.

fullonum, 217.

pilasus, 219.

sylvestris, 218.

Doronicum.

Pardalianches, 1236.

Draba. plantagineum H. 1236.

aizoides, 951.

hirta, 952.

incana, 953.

muralis, 954 .

rupestris, 952 . verna, 950 .

Drosera.

anglica, 497.

longifolia, 496 .

rotundifolia, 495 .

Dryas.

octopetala, 799 .

Echinochloa L. crus-galli L. 123.

Echinophora. spinosa, 418.

Echium. vulgare, 304 .

Elatine. Hydropiper, 1498. tripetala, 623 .

Eleochasis.

acicularis, 86. cepitosa H. 71 .

fluitans H. 73 .

multicaulis, 85 . palustris, 84.

Elymus. panciflora, 72 .

arenarius, 203. europeus, 205. geniculatus, 204.

Empetrum. nigrum. 1467.

Epilobium.

alpinum, 594

alsinifolium, 593

angustifolium, 586 . hirsutum, 587. montanum, 589 .

palustre, 592.

parviflorum, 588.

roseum, 590 ,

tetragonum, 591 .

Epimedium.

alpinum, 252.

Epipactis.

ensifolia, 1291.

grandiflora, 1290.

latifolia, 1287.

pallens W. 1290.

palustris, 1289.

purpurata, 1288.

rubra, 1292.

Erica.

ciliaris H. L. 606 .

cinerea, 604.

Daboecia IV. 626

Mediterranea, 1497.

Tetralix, 603.

Vagans, 605.

Erigeron.

acris, 1213.

alpinus, $1214^{\text {. }}$

canadensis, 1212.

uniflorus, 1215.

Eriocanlon.

septangulare, 1387.

Eriophorum.

alpinum, 89.

angustifolium, 92.

capitatum, 88 .

gracile, 93.

polystachion, 90 .

pubescens, 91 .

vaginatum, 87 .

Erodium.

cicutarium, 1019. maritimum, 1021. moschatum, 1020.

Erophila L. vulgaris L. 950 .

Eryngium.

campestre, 416.

maritimum, 415 .

Erysimum.

Alliaria, 994.

cheiranthoides, 993.

orientale, 995.

Sophia W. 990 .

Erythræa.

centauorium, 360 .

latifolia, 362,

littoralis, 361 . pulchella, 363 .

Ervum.

hirsutum, 1077. tetraspermum, 1076.

Euonymus. europous, 370 .

Eupatorium. cannabium, 1191. 
Euphorbia.

amygdaloides, 1309.

characias. 1310.

cyparissias, 1307.

Esula, 1306.

exigua, 1300.

helioscopia, 1304.

hiberua, 1307.

Lathyris, 1301.

paralia, 1303.

peplis, 1298.

peplus, 1299.

jlatyphylla H.L. 1305.

portlandica, 1302.

stricta, 1305.

Euphrasia.

officinalis, 918 .

Exacum.

filiforme, 244.

Fagus.

castanea, 1397.

Fedia.

sylvatica, 1398.

dentata, 58.

olitoria, 57 .

Festuca.

bromoicłes, 166.

calamaria, 170.

clumetorum W. 164.

duriuscula, 164.

elatior, 173.

fluitans W. 172.

gigantea, 169 .

loliacea, 171 .

myucus, 167 .

ovina, 162.

pinnata, 157.

pratensis, 172.

rubra, 165.

sylvatica, 174 .

uniglumis, 168 .

Filago. vivipara, 163.

Gallica, H. L. 1208.

Germanica, H. L.1210

minima, H. L. 1209.

Fæniculum H. L. vulgare H. L. 462.

Fragaria.

elatior, 783.

sterilis W. 794.

vesca $L . H . W, 784$.

Frankenia.

laevis, 560 .

pulverulenta, 561 .

Fraxinus.

excelsior, 16.

heterophylla, 17.

Fritillaria.

Meleagris, 511.

Fumaria.

capreolata, 1045.

claviculata, 1042. littea, 1041.

officinalis, 1043 .

parvifiora, 1044.

solida, 1040.

Gagea H. L.

lutea H. L. 513 .

Galanthus.

nivalis, 499.

Galeobdolon.

luteum, 892 .

Galeopsis.

angustifolia L. 888.

Lactanum, 888.

Tetrahit, 890 .

versicolor, 891 .

Galium. villosa, 889 .

anglicum, 240 .

aparine, 242.

aristatum, 233.

boreale, 241.

cinereum, 232.

eruciatum, 226.

erectum, 231.

Mollugo, 239.

pallustre, 227.

parisiense H. L. 240.

pucillum, $23 \overline{7}$.

saccharatum H. 242.

saxatile, 229 ,

spurium, 236.

tricorne, 235.

uliginosum, 230.

verrucosum, 234.

verum, 238.

Witheringii, 228.

Gastridium H. L.

lendigerum H.L. 113.

Genista.

anglica, 1050.

pilosa, 1049 .

tinctoria, 1048.

Gentiana.

acaulis, 410 .

amarella, 413 .

campestris, 414.

nivalis, 412.

pneumonathe, 409 . verna, 41 ?.

\section{Geranium.}

columhinuın, 1033.

dissectum, 1032.

lucidum, 1027.

molle, 1028 .

nodosum, 1023.

phæum, 1022.

pratense, 1025.

pyrenaicum, 1030.

prostratum L. 1034 .

pusillum, 1029.

robertianum, 1026.

rotundifolia, 1031.

sanguineum, 1034. 
reum.

sylvaticum, 1024.

rivale, 798 .

u rbanum, 797.

Glaucium.

corniculatum L. 804.

luteum, 803.

phœniceum W. H. 804.

Glaux. violaceun, 805 .

maritima, 379 .

Glechoma. hederacea, 882.

Glyce L. maritima L. 955 .

Glyceria.

aquatica, 140 .

distans, 142.

fluitans, 141.

maritima, 143 .

procumbens, 144. rigida, 145 .

Gnaphalium.

dioicum, 1203.

Gallicum, 1208.

Germanicum, 1210.

luteo-album, 1201.

margaritaceum, 1ะ02.

minimum, 1209.

rectum, 1205.

supinum, 1206.

sylvaticum, 1204 . uliginosum, 1207.

Goodyera.

repens, 1281.

Gymnadenia W. H. L. conopsea W.H.L. 1273

Habenaria W. H.

albida W. H. 1269.

bifolia W. H. 1260

viridis W. H. 1270 .

Hedera.

Helix, 377.

Hedysarum.

Onobrychis, 1080.

Helianthemum.

guttatum H. L. 819.

ledifolium H. L. 820 .

polifolium H. 824 .

surrejanum L. 821.

vulgare $H$. L. 822 .

Heleocharis L.

acicularis L. 86 .

ccespitosa L. 71.

multicaulis L. 85 .

palustris L. 84 .

pauciflora L. 72.

Heleogitan L.

fluitans L. 73.

Helleborus.

fœtidus, 857 .

viridis, 856 .

Helnuinthia H. L. echioides H. L. 1126.

Helosciadium H. L.

inundatum H. L. 493.

nodiflorum H. L. 437 .

repens $H$. L. 438 .

Heracleum.

Sphondylium, 476.

Herminium.

Monorchis, 1275.

Herniaria.

glabra, 383.

hirsuta, 381 .

Hesperis.

matronalis, 999.

Hieracium.

alpinum, 1142.

aurantiacum, 1146.

auricula, 1145 .

corinthoides, 1154 .

denticulatum, 1158.

dubium, 1144.

Halleri H. L. 1156.

Lawsoni, 1151.

maculatum, 1148.

molle, 1153.

murorum, 1147.

paludosum, 1152.

Pilosella, 1143.

prenanthoides, 1159.

pulmonarium, 1150.

Sabandum, 1157.

sylvaticum, 1149.

Taraxaci W. 1140.

umbellatum, 1160.

villosum, 1155.

Hierochloe.

borealis, 135.

Hippocrepis. comosa, 1079.

Hippophae. rhamnoides, 1470.

Hippuris. vulgaris, 5.

Hirculus L. ranunculoides L. 643.

Holcus.

alpina W. 191.

avenaceus, 134.

fatna W. 187.

flavescens W. 192.

lanatus, 132 .

mollis, 133.

pratensis W. 190.

pubescens W. 189 strigosa W. 188.

Holdschcenus L. vulgaris $\mathrm{L} .76$.

Holosteum. umbellatum, 215.

Hordeum.

marinum, 208. maritimum, 208. murinum, 206. 
nodosum W. 207. pratense, 207.

$\mathrm{F}$,ttonia. palustris, 313 .

fumulus.

Lupulus, 1472.

Hutchinsia. petræa, 961 .

Hyacinthus. nonscriptus H.L. 520 . racemosus, 521.

Hydrocharis.

Morsus Ranæe, 1481 .

Hydrochloa L. aquatica L. 140.

Hydrocotyle. vulgaris, 472 .

Hyoseyamus. niger, 356.

Hypericum.

Androsœmum, 1114.

barbatum, 1120.

calycinum, 1113.

delplinense L. 1117.

dubium, 1117.

elodes, 1123.

hirsutum, 1121.

humifusum, 1118.

montanum, 1119.

perforatum, 1116.

pulehrum, 1122.

quadrangulum, 1115.

Hypocharis.

glabra, 1166.

maculata, 1165.

radicata, 1167 .

1beris.

Ilex.

amara, 974.

Aquifolium, 261.

Illecebrum.

verticillatum, 378 .

Impatiens. noli-me-tangere, 340 .

Imperatoia. ostruthium, 456.

Inula Conyza H. 1211. crithmoides, 1233.

Helenium, 1230.

Iris. pulicaris, 1232.

fœtidissima, 65 .

Pseud-acorus, 64.

Isatis.

tinetoria, 975.

Isnardia. palustris, 256.

Isolepis $\mathrm{L}$. setacea L. 77.

Jasione. montana, 337 .

Juncus. acutiflorus, 584. acutus, 530.

araticus, 536.

Balticus H. 536.

biglumis, 545 .

bufonius, 542 .

capitatus, 544 .

castaneus, 547.

cœnosus, 540.

compressus, 539 .

conglomeratus, 533.

effusus, 534.

filiformis, 535 .

Gesneri, 541.

glancus, 532.

gracile IV. 544.

lampocarpus, 549 .

maritimus, 531 .

obtusiflorus, 550 .

pilosus W. L. 552 .

polycephalus, $55 \mathrm{l}$.

spieatus L. 557.

squarrosus, 538.

ternuis H. 541 .

trifidus, 537.

triglumis, 546 .

uliginosus, 543 .

Juniperus.

communis, 1482.

nana, 1483.

Knappia. agrostidea, 109.

Knautia H. L. arvensis H. L. 221.

Kobresia.

caricina, 1377.

Koniga $\mathrm{H}$.

maritima H. 955 .

Lactuca.

muralis H. 1135.

saligna, 1134.

Scariola, 1133.

virosa, 1132.

Lagurus.

ovatus, 193.

Lamium.

album, 883.

amplexicaule, 887 .

incisum, 886 .

intermedium H. 837 .

maculatum, 884 .

purpureum, 885 .

Lappa L.

glabra L. 1171.

tomentosa L. 1172.

Lapsana.

communis, 1168. pusilla, 1169 .

Larbrea L. aquatica L. 680 .

Lathræa. squamaria, 923.

Lathyrus. Aphaca, 1059. 
hirsutus, 1061.

latifolius, 1064.

maritimus, 1055.

Nissolia, 1060.

palustris, 1065 .

pratensis, 1062.

sylvestris, 1063.

Lavatera.

arborea, 1039.

Leiogyne L.

aizoides L. 644 .

cernua L. 646.

granulata L. 645 .

nivalis L. 641 .

rivularis L, 647 .

Lemna.

gibba, 46.

minor, 45.

polyrhiza, 47.

trisulca, 44.

Leontodon.

autumnale W. 1141.

hirtum W. 1139.

hispidum W. 1138.

palustre, 1137.

Taraxacum, 1136.

Leonorus.

Cardiaca, 901.

Lepidium.

campestre, 959.

Draba, 1501 .

hirtum, 960.

latifolium, 957.

petroeum W. 961 .

ruderale, 958 .

Smithii H. 960.

Leucojum.

æstivum, 500.

Ligusticum.

cornubiense, 460 .

scoticum, 459 .

Ligustrum.

vulgare, 15.

Limbarda.

tricuspis, 1233.

Limosella. aquatica, 941.

Linaria H. L.

Cymbalaria H. L. 926.

Elatine H. L. 928.

minor H. L. 931.

repens H. L. 929 .

spuria H. L. 927.

vulgaris H. L. 930 .

Linnæa.

borealis, 939.

Lynosiris $\mathrm{H}$. vulgaris H. 1192.

\section{Linum.}

angustifolium, 492.

catharticum, 493.

perenne, 491.

nsitatissimum, 490.
Liparis H. L.

Loeseli H. L. 1294.

Listera.

cordata, 1285.

Nilus Avis, 1286.

ovata, 1284.

Lithospermum.

arrense, 290.

matitimum, 292.

officinale, 298.

purpuro-cæruleum,291

Lit torella.

lacustris, 1378.

Lobelia.

Dortmanna, 338.

urens, 339 .

Lolium.

arvense, 201.

bromoides W. 168.

perenne, 199.

temulentum, 200.

Lonicera.

caprifolium, 365 .

Periclymenum, 366.

Lotus.

Xylosteum, 367.

angustissimus, 1106.

corniculatus, 1103 .

decumbens, 1105.

major, 1104

tenuis H. 1105.

Luciola (Luzula of H.\&L.)

arcuata, 558.

campestris, 555 .

congesta, 556 .

Forsteri, 553.

linigar W. 556 .

pilosa, 552.

spicata, 557.

sylvatica, 554 .

Lychnis.

alpina, 712.

dioica, 713 .

Flos Cuculi, 710.

viscaria, 711 .

sylvestris L. 713 .

vespertina L. 713.

Lycopsis.

arvensis, 303.

Lycopus.

europæus, 48.

Lysimachia.

nemorum, 316.

nummularia, 317.

thyrsiflora, 315.

vulgaris, 314.

Lythrum.

hyssopifolium, 728 .

Malaxis.

Salicaria, 727.

Loeselii, 1294.

Malva. 
moschata, 1038. rotundifolia, 1037. sylvestris, 1036 .

Marrubium.

Maruta. vulgare, 900 . foetida, 1247.

Matricaria. Chamomilla, 1243.

Matthiola. incana, 997. sinuata, 998 .

Meconopsis. cambrica, 811.

Medicago. denticulata H. 1110. falcata, 1108. lupulina, 1109. maculata, 1110. minima, 1112. muricata, 1111. sativa, 1107.

Melampyrum. arvense, 992. cristatum, 991. pratense, 993. Melica. sylvaticum, 994.

corulea, 138. nutans, 137. uniflora, 136.

Melilotus L. H. officinale T. H. 1085. Melittis. grandiflora, 909.

Mentha. Mlelisophyllum, 908 .
acutifolia, 875 .
agrestis, 880 . arvensis, 879 . eitrata, 873 . gentilis, 877 . gracilis, 878 . hirsuta, 874 . piperita, 872 . precox W. 879 . pulegium, 881 . rotundifolia, 870 . rubra, 876. sylvestris, 869 . viridis, 871 .

Menyanthes. nymphœoides, 312. trifoliata, 311.

Menziesia. cœrulea, 600 . polifolia, 601 .

Mercurialis. annua, 1480. Mespilus. perennis, 1479 . cotoneaster, W. 740 . germanica, 739.

Meum. oxyacantha, 738 . athamanticum, 461.

Milium. Fœniculum, 462.

effusum, 112. lendigerum, 113.

Mœnchia. erecta, 279. glauca L. 279.

Monotropa.

Montia. Hypopitys, 625.

fontana, 214.

Myosotis.

alpestris, 286. arvensis, 287. exspitosa, 283. collina H. 287. intermedia, 284. palustris, 288. sylvatica, 285. versicolor, 288.

Myosurus. minimus, 498 .

Myrica. Gale, 1471.

Myriophyllum. spicatum, 1390.

Myrrhis. verticilatum, 1397.

aromatica, 433. aurea, 432. odorata, 430 . temulenta, 431 .

Narcissus. biflorus, 502 . poeticus, 501 .

Nardus. pseudo-narcissus. stricta, 94.

Narthecium. ossifragum, 523.

Nasturtium. amphibium, 987. officinale, 984 . sylvestre, 985 .

Neottia. terrestre, 986. gemmipara, 1283. nidus-avis L. 1286. spiralis, 1282.

Nepeta. cataria, 867 .

Nuphar.

lutea, 813. minima W. 814 . pumila, 814 .

Nymphæa. alba, 812 .

Enanthe. crocata, 450. fistulosa, 447 . 
peucedanifolia, 449 . phellandrium, 451 . pimpinelloides, 448 .

Enothera. biennis, 585.

Onobrychis $\mathrm{H}$. L. sativa H. L. 1080.

Ononis.

arvensis, 1500. spinosa W. L. 1053.

Onopordum. Acanthium, 1187.

Opbiurus. incurvatus, 202.

Ophrys.

anthropophora W.127t apifera, 1277. alachnites, 1278. aranifera, 1279. colallorhiza, W. 1295. cordata, IV. 1285. fucifera, 1280 . gemmifera, W. 1283. Loselii, W. 1294. monorchis, W. 1275 . muscifera, 1276. nidus avis, W. 1286. ovata, W. 1284. spralis, W. 1282.

Orchis.

albida, 1269

bifolia, 1260.

conopsea, 1273.

fusea, 1265.

hireina, 1268.

latifolia, $127 \mathrm{I}$.

maculata, 1272.

macra, H. 1267.

mascula, 1263.

militaris, 1266.

morio, 1262.

pyramidalis, 1261.

tephrosanthos, 1267.

ustulata, 1264.

viridis, $12 \% 0$.

Origanum,

vulgare, 903.

Ornith ogalum.

luteum, 513.

nutans, 516.

pyrenaicum, 514.

umbellatum, 515 .

Ornithopus. perpusillus, 1078 .

Orobanche. corulea, 946

elatior, 943

major, 942 .

minor, 944 .

ramosa, 947.

rubra, 945.

Orobus.

niger, W. H. L. 1057. sylvaticus, 1058 . tuberosus, 1056.

Oxalis.

Acetosella, 707.

corniculata, 708 .

Oxycoccus, $\mathrm{L}$.

palustris, L. 599.

Oxyria.

reniformis, 563 .

Oxytropis.

campestris, 1084.

uralensis, 1083.

Preonia.

corallina, 825 .

Panicum.

crus-galli, 123.

Dactylon, L. 119.

sanguinale, W. 120 .

verticillatum, 121 .

viride, 122.

Papaver.

argemone, 807.

cambricum, 811 .

dubium, 808 .

hybridum, 806 .

rboeas, 809.

somniferum, 810 .

Parietaria.

officinalis, 255.

Paris.

quadrifolia, 621 .

Parnassia.

palustris, 486 .

Pastinaca.

sativa, 475

Pedicularis.

palustris, 924. sylvatica, 925 .

Peplis.

portula, 562.

Petasites, $\mathbf{H}$.

vulgaris H. 1217.

Petroselinum H. L.

sativum, H. 1496.

segetum, H. L. 442.

Puecedanum.

officinale, 474.

Ostruthium H.L. 456. palustre. H. L. 473 .

Phalaris.

arundinacea, 96 .

canariensis, 95.

phleoides, W. 100 .

Phleum.

alpinum, 98.

arenarium, 102 ,

asperum, 99.

Boehmeri, 100.

Michelii, 101.

pratense, 97.

Physospermum H. L.

commutatum, L. 460 . cornubiense, H. 460 . 
Phyteuma.

orbiculare, 336. spicata, 1494.

Picris.

echioides, 1126.

hieracioides, 1227.

Pimpinella.

dioica, 467 .

magna, 466.

saxifraga, 465 .

Pinguicula.

alpina, $\mathrm{H}$.

grandiflora, 40.

Iusitanica, 38.

Pinus. vulgaris, 39 .

Pisum. sylvestris, 1403.

maritimum, 1055.

Pluntago.

coronopus, 249.

lanceolata, 247.

major, 245.

maritima, 248.

media, 246.

Platanthera, L.

albida, L. 1269.

bifolia, L. 1260.

Poa. viridis, L. 1270.

alpina, 147.

annua, 152.

aquatica, H. W. 140.

bulbosa, 149 .

compressa, 146.

cristata, W. 124.

distans, W. L. 142.

flexuosa, W. 148.

fluitans, H, 141.

glauca, 153.

laxa, 148.

maritima, W. H. 143.

nemoralis, 154.

pratensis, 150.

procumbens, W.H.140

rigida W. H. 145.

trivialis, 150.

Polemonium. cœruleum, 325.

Polycarpon. tetraphyllum, 216.

Polygala. vulgaris, 1046.

Polygonum.

amphibium, 61].

aviculare, 618 .

bistorta, 616 .

convolvulus, 620 .

Fagopyrum, 619.

Hydropiper, 614.

lapathifolium, 613.

minus, 615 .

persicaria, 612.

viviperum, 617.

Polypogon.

jittoralis, 111 .

monspeliensis, 110.

Populus.

alba, 1474.

canescens, 1475 .

nigra, 1477 .

tremula, 1476 .

Potamogeton.

acutifolius, H. 273.

compressus, 270.

crispus, 269.

cuspidatus, 271.

densus, 265.

fluitans, 266.

gramineus, 272.

heterophyllus, 263.

lanceolatus, 263.

lucens, 267.

natans, 262.

obtusifolius L. 272.

pectinatus, 274.

perfoliatus, 264.

proteus, L. 267.

pusillus, 273.

rufescens, L. H. 266.

Potentilla.

alba, 791.

alpestris, 788 .

anserina, 785 .

argentea, 787 .

comarum, L. 800 .

Fragaria, 794.

Fragariastrum $\$ 91$.

fruticosa, 784 .

opaca, 790 .

reptans, 792.

rupestris, 786 .

Termentilla L. 795.

tridentata, 793 .

verna, 789 .

Poterium. sanguisorba, 1394.

Prenanthes. hieracifolia, L. 1162 .

Primula. muralis, 1135 .

elatior, 306.

farinosa, 308 .

scotica, 309 .

veris, 307 .

vulgaris, 305 .

Prismatocarpus. hybridus, L. 334.

Prunella.

vulgaris, 912.

Prunus.

cerasus, 734 .

domestica, 735 .

insititia, 736 .

Padus, 733.

spinosa, 737. 
Pulicaria. dysenterica, H.L. 1231 vulgar is $H$. L. 1232.

Pulmoraria.

angustifolia, 298.

maritima, W. 292. officinalis, 297.

Pyrethrum. inodorum, 1241. maritimum, 1242. Parthenium, 1240.

Pyrola.

media, 631.

minor, 632.

rotundifolia, 630 .

secunda, 633 .

Pyrus. uniflora, 634 .

aria, 747 .

aucuparia, 745 .

communis, 741 .

domestica, 744 .

hybrida, W. 747.

intermedia, L. 747 .

Malus, 742.

pennatifida, 746 .

Quercus.

torminalis, 743 .

robur, 1395.

sessiliflora, 1394.

Radiola.

linoides, L. 281.

millegrana, 281 .

Ranunculus.

acris, 850 .

alpestris, 846 .

aquatilis, 854 .

arvensis, 851 .

auricomus, 844 .

bulbosus, 847 .

Ficaria, 843.

Flammula, 840 .

gramineus, 842 .

hederaceus, 853 .

hirsutus, 848 .

lingua, 841 .

parviflorus, 852 .

Philonotis, L. 848.

repeus, 849 .

sceleratus, 845 .

Raphanus.

maritimus, 10.8 .

Reseda.

Raphanistrum, 1017.

lutea, 731.

luteola, 730 .

Rhamnus.

catharticus, 368 .

frangula, 369 .

Rhinanthus.

crista-galli, 916.

Rhodiola. rosea, 1478.

Rhyncospora.

alba, 67 .

fusca, 68 .

Ribes.

alpinum, 374.

Grossularia, 376.

nigrum, 375.

petræum, 372 .

rubrum, 371.

spicata, 373 .

Robertsonia, L.

geum, L. 637.

hirsuta, L. 638.

stellaris, L. 640 .

umbrosa, L. 639 .

Römeria, L.

Rosa.

hybrida, L. 805.

arvensis, 770 .

Borreri, 762 .

cæsia, 763.

canina, 768 .

cinnamomea, 751.

collina, L. 766 .

Doniana, 755 .

dumetorum, 765 .

Forsteri, 766.

glancophylla, W. 764 .

gracilis, 756 .

hirbenica, 767 .

inodorum, 765 .

involuta, 754 .

micrantha, 761 .

mollis, L. 758 .

rubella, 752 .

rubiginosa, 760 .

Sabina. 757.

sarmentacea, 764 .

sepium, H. 760 .

spinosissima, 753.

sylvestris, L. 759 .

systyla, 769 .

tomentosa, 759 .

villosa, 758 .

Rottbolia.

incurrata, 202.

Rubia.

Rubus.

peregrina, 243.

affinis, 1506.

areticus, 780 .

cæsius, 778.

Chamæmorus, 781.

corylifolius, 777 .

fruticosus, 771 .

idæus, 776 .

leucostachys, 774 .

plicatus, 772 .

rhamnifolius, 773 .

saxatalis, 779 .

suberectus, 775 .

Rumex. 
acetosa, 572.

acetosella, 573 .

acutus, 566 .

crispus, 565 .

glomeratus, L. 566 .

Hydrolapathum, 571 .

maritimus, 569.

nemolapathum, L.564.

obtusifolius, 567 .

palustris, 570 .

pulcher, 568.

Ruppia. sanguilleus, 564 .

Ruscus.

maritima, 275.

Sagina.

apetala, 278.

cerastoides, W. 717 .

maritima, 277.

procumbens, 276 .

Sagittaria.

sagittifolia, 1392.

Salicornia.

fruticosa, 4.

herbacea, 1.

procumbens, 2.

Salix.

radicans, 3 .

acuminata, 1462.

alba, 1466.

amygdalina, 1407.

Andersolliana, 1458.

angustifolia, H. 1436.

aquatica, 1453.

arbuscula, 1436.

arenaria, 1441.

argentea, 1443.

aurita, 1452 .

bicolor, 1416.

Borreriana, 1411.

caprea, 1461.

carinata, 1435.

cinerea, 1451.

chrysanthos W. 1442.

cotinifolia, 1455.

Croweana, 1429.

Damascena H. 1458.

Davalliana, 1413.

decipiens, 1421.

Dicksoniana, 1434.

Doniana, 1449.

fissa W. 1427 .

foetida, 1444.

Forbiana, 1427.

Forsteriana, 1459.

fragilis, 1422.

fusca, 1446.

glauca, 1439.

hastata H. 1418.

Helix, 1425.

herbacea, 1437.

irirta, 1456.
Hofimanniana, 1405. incubacea, 1448.

Lambertiana, 1426.

lanata, 1442.

lanceolata, 1406.

laurina H. 1416.

malifolia, 1418.

mollissima W. 1466

myrsinites, 1433.

nigricans, 1409 .

nitens, 1412.

oleifolia, 1454.

pentandra, 1408.

petiolaris, 1419.

phylicifolia, 1410.

plostrata, 1447.

prunifolia, 1430 .

purpurea, 1424.

radicans $H .1410$.

repens, 1445.

reticulata, 1438 .

rosemarinifolia, 1450 .

rubra, 1428.

rupestris, 1457.

Russelliana, 1423.

Smithiana, 1466.

sphacelata, 1460.

stipularis, 1465.

Stuartiana, 1440.

tenuifolia, 1417.

tetrapla, 1415.

triandria, 1404.

undulata H. 1406.

vaccinifolia, 1431.

venulosa, 1432.

viminalis, 1463.

vitellina, 1420 .

Salsola.

Wulfeniana, 1414.

fruticosa, 400 .

Salvia. kali, 399.

pratensis, 49 .

verbenacea, 50 .

Sambucus.

Ebulus, 481.

nigra, 482.

Samolus.

valerandi, 364.

Sanguisorba.

officinalis, 251

Sanicula. europæa, 417.

Saponaria. officinalis, 659 .

Satyrium W. albidum W. 1269.

hircinum W. 1268.

viride W. 1270 . repens $W$.

Saxifraga.

aizoides, 644 .

cœspitosa, 651 . 
cernua, 646 .

elongella, 655 .

Geum, 637.

granulata, 645 .

Hirculus, 643 .

hirsuta, 638 .

hirta, 652.

hypnoides, 654 . muscoides, 649 .

nivalis, 641 .

oppositifolia, 642 .

pedatifida, 656 .

platypetala, 653.

pygmea, 650 .

rivularis, 647 .

stellaris, 640.

tridactylites, 648 .

umbrosa, 639 .

Scabiosa.

arvensis, 221.

columbaria, 222 .

succisa, 220 .

Scandix.

Pecten-Veneris, 427

Schedonorus L.

elatior L. 173.

loliaceus L, 17].

pratensis L. 172.

sylvaticus L. 174 .

Schoenus.

nigracans, 66 .

rufus L. 79 .

Scheuchzeria.

Scilla. palustris, 574 .

autumnalis, 519 .

bifolia, 518 .

nutans, 520 .

verna, 5$] 7$.

Scirpus.

acicularis W. 87

caspitosus, 71 .

caricinus, 78

carinatus, 81 .

fluitans, 73.

glaucus, 75 .

Holoschoenus, 76.

lacustris, 74 .

maritimus, 82 .

pauciflorus, 72.

rufus, 79 .

setaceus, 77 .

sylvaticus, 83

triqueter, 80 .

Sclerantlius.

annuus, 657.

perennis, 658 .

Selerochloa.

maritima, 143.

procumberss, 144.

rigida, 145 .

Serophularia

aquatica, 935. nodosa, 934.

Scorodonia, 936.

vernalis, 937.

Scutellaria.

galericulata, 910.

minor, 911.

Sedum.

albescens L. 704 .

acre, 699.

album, 702 .

anglicum, 698 .

dasyphyllum, 697 .

Forsterian um, 706 .

glaucum, 704 .

reflexum, 703.

Rhodiola L. 1478.

rupestre, 705 .

sexangulare, 700 .

Telephium, 696 .

villosum, 701 .

Selinum.

palustre, 473 .

Sempervivum. tectorum, 732.

Senebiera.

coronopus, 972.

didyma, 973.

Senecio.

aquaticus, 1225 .

Jacobea, 1224.

lividus, 1220.

paludosus, 1226.

saracenicus, 1227.

squalidus, I 222.

sylvaticus, 1221.

tenuifolius, 1223.

viscosus, 1219 .

vulgaris, 1218 .

Serapias W.

ensifolia W. 1291.

grandiflora W. 1290 .

latifolia W. 1287.

palustris W. 1289 .

rubra W. 1292.

Serratula.

alpina, 1174.

tinctoria, 1173.

Seseli H. L.

libanotes H. L. 464 .

Sesleria.

cærulea, 139

Setaria H. L

verticilata H. L. 121.

viridis H. L. 122 .

Sherardia.

arvensis, 223.

Sibbaldia. procumbens, 494 .

Siluthorpia. europæa, 940 .

Silaus H. L. pratensis H. L. 468 .

Silene. 
acaulis, 674 .

anglica, 665 .

Armeria, 671.

conica, 669 .

inflata, 667.

Italica $\mathrm{H}$.

maritima, 668.

noctifiora, 670 .

nutans, 672 .

otites, 673 .

quinquevulnera, 666.

Silybum L.

marianum L. 1178.

Sinapis.

alba, 1013.

arvensis, 1012 .

incena $\mathrm{H}$

muralis, 1016 .

nigra, 1014.

Sison.

tenuifolius, 101 j.

amomum, 411.

peucedarsifolium W.443.

Phellandrium W 451 .

inundatum W. 439.

segetum, 442.

verticillatum W, 440 .

Sisymbrium.

Irio, 989.

officinale, 988.

Sophia, 990.

thalianum H. 1000.

Siuın.

angustifolium, 436.

erectum L. 436 .

inundatum, 439 .

latifolium, 435.

nodiflorum, 437.

repens, 438.

Smyrnium.

Solanum.

Olusatrum, 453.

Dulcamara, 358 nigrum, 359 .

Solidago. virgaurea, 1229.

Sonchus. alpinus H. L. 1128 . arvensis, 1130. coruleus, 1128. oleraceus, 1131 . palustris, 1129.

Sparganium.

natans, 1318 .

ramosum, 1315

simplex, 1317.

Spartina.

stricta, 159

Spartium.

seoparium, 1047.

Spergula.

arvensis, 72.2.

nodlosa, 723 . saginoides, 724.

subulata, 725 .

Spirzea.

filipendula, 749

salicifolia, 748 .

Ulmaria, 750 .

Spiranthes L.

autnmualis L. 1282 .

gemmipara L. 1203.

Stachys.

ambigua, 895 .

arvensis, 898 .

germanica, 897.

palıstris, 896 .

sylvatica, 894 .

Staphylea.

pinnata, 483 .

Statice.

Armeria, 487.

Limonium, 488

plantiginea $\mathrm{H}$.

reticulata, 489 .

spatluulata, 488 .

Stellaria.

cerastoides, 682.

glauca, 679 .

graminea, 678.

holostea, 677 .

media, 676 .

nemorum, 675.

scapigera, 681 .

Stipa. uliginosa, 680 .

[ennata, 186.

Stratioies.

aloides, 829 .

Subularia.

aquatica, 949 .

Swertia.

perennis, 408.

Symphytum.

officinale, 299.

tuherosum, 300 .

Tamarix.

gallica, 484 .

Tamus.

communis, 1473.

Tanacetum.

Taxus. vulgare, 1194.

baccata, 1484 .

Teesdalia.

Iberis L. 974

nudicaulis, 962.

Teucrium.

chamædrys, 8rif. scorodonia, 864 .

Scordium, 865.

Thalietrum.

alpinum, 835.

flavum, 838 .

ınajus, 837 .

minus, 836 . 
Thesium.

linophyllum, 380.

Thalaspi.

alpestre, 965.

arvense, 963.

Bursa Pastoris, 966. perfoliatum, 964 .

Thrincia H. L.

hirta H. L. 1139.

Thymus.

Acinos, 905.

Calamintha, 906

Nepeta, 907.

Tilia.

Serpyllum, 904.

europæea, 815 .

yrandiflora, 816 .

intermedia L. 815 .

Tillaa. parviflora, 817.

Tofieldia.

muscosa, 280.

Tordylium.

palustris, 575 .

maximum, 478.

officinale, 477 .

Torilis.

Anthriscus, 423.

infesta, 424 .

nodosa, 425 .

Tormentilla.

officinalis, 795 .

reptans, 796.

Tragopogon. parvifolius, 1125. pratensis, 1124.

Trichodium.

caninum, 115.

setaceum, 116.

Trichonema.

bulbocodium, 63 . columna $\mathrm{H}$.

Trientalis. europæa, 584.

Trifolium.

arvense, 1095.

filiforme, 1102 .

fragiferum, 1099.

glomeratum, 1097.

maritimum, 1093.

medium, 1092.

minus, 1101.

ochroleucum, 1090.

officinale, 1085.

ornithopodioides, 1086.

pratense, 1091.

procumbens, 1000.

repens, 1087.

resupinatum, 1503.

scabrum, 1096

squarrosum L. W. 1090

stellatum, 1094.

striatum, 1098. subterraneum, 1089.

suffocatum, 1088.

Triglochin.

maritimum, 577.

palustre, 576 .

Trigonella, L.

ormithopodioides, L. 1086.

Trinia, H. L.

Triodia. glaberrima, H. L. 467.

decumbens, 155.

Triticum.

caninum, 211.

cristatum, 212.

junceum, 209.

loliaceum, 213.

repens, 210 .

Trollius.

Tulipa.

europæus, 855 .

sylvestris, 512 .

Turritis. glabra, 1006.

Tussilago.

Farfara, 1216.

Typha.

Petasites, 1217.

angustifolia, 1313.

latifolia, 1312.

minor, 1314.

Ulex.

europæus, 1051.

nanus, 1052 .

Ulmus.

campestris, 401.

glabra, 405.

major, 403 .

montana, 404.

suberosa, 402.

Umbilicus, L.

erectus, L. 694.

Urtica. pendulinus, L. 693 .

dioica, 1383.

pilulifera, !381.

repens, $\mathrm{L}$.

urens, 1382.

Utricularia.

intermedia, 42.

minor, 43.

vulgaris, 41 .

Vaccinium.

Myrtillus, 596.

oxy coccos, 599 .

uliginosum, 597.

vitis 1dæa, 598 .

Valeriana.

dioica, 54 .

officinalis, 55 .

pyrenaica, 56 .

rubra, 53.

Valerianella.

denta ta, 58. 
Vella.

olitoria, 57.

annua, 948.

Verbascum.

Blattaria, 354.

lychnites, 350 .

pulverulentum, 351.

nigrum, 352.

thapsus, 349 .

Verbena.

virgatum, 353.

officinalis, 868 .

Veronica.

agrestis, 33.

alpina, 24.

anagallis, 27.

arvensis, 34.

Beccahunga, 26.

Chamædrys, 31 .

fruticulosa, 22.

hederifolia, 35 .

hirsuta, 30.

hybrida, 21.

montana, 32 .

officinalis, 29.

polita, H. 33.

saxatilis, 23.

scutellata, 28.

serpyllifolia, 25.

spicata, 20.

triphyllos, 36 .

verna, 37 .

Viburnum.

lantana, 479.

Vicia.

opulus, 480 .

angustifolia, 1069. bithynica, 1075.

cracea, 1067 .

hybrida, 1072.

lovigata, 1073.

lathyroicles, 1070.

lutea, 1071.

sativa, 1068.

sepium, 1074.

svlvatica, 1066 .

Villarsia, L. H. nymphaoides, L. H. 312 .

Vinca.

major, 382.

minor, 381.

Viola.

canina, 344.

flavicornis, 346.

grandiflora, W. 348.

hirta, 341.

lactea, 345.

lutea, 348 .

odorata, 342 .

palustris, 343 .

tricolor, 347 ,

Viscum.

album, 1469.

Vulpia, L.

bromoides, L. 166.

Myurus, L. 167.

uniglumis, L. 168.

Xanthium.

strumarium, 1384.

Zannichellia.

palustris, 1311.

Zostera.

marina, 6. 


\title{
LATIN INDEX,
}

ARRANGED ACCORDING TO THE LINNAAN SYSTEM, AND IN THE ORDER

\author{
OF THE PLATES.
}

MONANDRIA.

\section{Monogrnia.}

\section{Salicornia.}

herbacea, 1.

procumbens, 2.

raclicans, 3 .

fruticosa, 4.

Hippuris.

vulgaris, 5 .

Zostera.

marina, 6.

Chara.

vulgaris, 7 .

hispida, 8.

flexilis, 9.

translucens, 10 .

- .nidifica, 11.

gracilis, 12.

Digynia.

Callitriche.

verna, 13

autumnalis, 14.

DIANDRIA.

\section{Monogyia.}

Ligustrum.

vulgare, 15.

Fraxinus.

excelsior, 16.

Circea. heterophylla, 17 .

lutetiana, 18 . alpina, 19.

Veronica.

spicata, 20.

hybrida, 21.

fruticulosa, 22.

saxatilis, 23 .

alpina, 24.

serpyllifolia, 25 .

Beccabunga, 26.

Anagallis, 27 .

scutellata, 28.

officinalis, 29.

hirsuta, 30 .

chamœdrys, 31. montana, 32 .

agrestis, 33 .

arvensis, 34 .

hedlerifolia, 35 .

triphyllos, 36 .

verna, 37 .

Pinguicula.

lusitanica, 38.

vulgaris, 39.

grandiflora, 40.

Utricularia.

vulgaris, 41.

intermedia, 42.

minor, 43 .

Leinna.

trisulca, 44.

minor, 45.

gibba, 46.

polyrhiza, 47.

Lycopus.

europæus, 48.

Salvia.

pratensis, 49.

verbenaca, 50 .

Cladium. mariseus, 51.

Digyeia.

Anthoxanthum. odoratum, 52.

TRIANDRIA.

MONOGYNIA.

Valeriana.

rubra, 53.

dioica, 54 .

officinalis, 55 .

Fedia. pyrenaica, 56 .

olitoria, 57 .

dentata, 58 .

Crocus.

sativus, 59.

vernus, 60 .

reticulatus, 61 .

nudiflorus, 62 .

Trichonema. 
Iris.

Bulbocodium, 63 .

Pseud-acorus, 64.

Schœnus.

nigricans, 66 .

Rhynchospora.

alba, 67 .

fusea, 68 .

Cyperus.

longus, 69 .

Scirpus.

fuscus, 70 .

eæspitosus, 71 .

paucifiorus, 72 .

fluitans, 73 .

lacustris, 74 .

glaucus, 75.

Holosehœenus, 76 .

setace us, 77.

caricinus, 78.

rufus, 79 .

triqueter, 80 .

carinatus, 81.

maritimus, 82 .

Eleocharis. sylvaticus, 83 .

palustris, 84 .

multicaulis, 85 . acicularis, 86.

Eriophorum.

vaginatum, 87 .

eapitatum, 88 .

alpinum, 89.

polvstachion, 90 .

pubescens, 91 .

angustifolium, 92.

Nardus. racile, 93.

stricta, 94.

Digynia.

Phalaris.

canariensis, 95 .

arundinacea, 96.

Plsleum.

pratense, 97 .

alpinum, 98.

asperum, 99 .

Boehmeri, 100.

Mlichellii, 101.

arenarium, 102.

Alopecurus.

pratensis, 103.

alpinus, 104.

agrestis, 105.

bulbosus, 106 .

geniculatus, 107.

Knappia. fulvus, 108 . agrostidea, 109.

Polypogon.

monspeliensis, 110.

littoralis, 111 .
Milium.

effusum, 112.

lendigerum, 113.

Agrostis.

Spica venti, 114.

canina, 115.

setacea, 116.

vulgaris, 117.

alba, 118.

Cynodon.

Dactylon, 119.

Digitaria.

sanguinalis, 120 .

Panicum.

verticillatum, 121.

viride, 122.

Aira.

Crus-galli, 123.

cristata, 124.

cæspitosa, 125.

alpina, 126 .

flexuosa, 127. canescens, 128. Sompe/1/ioney

præcox, 129.

caryophyllea, 130.

aquatica, 131.

Holcus.

lanatus, 132.

mollis, 133.

avenaceus, 134.

Hierochloe.

borealis, 135 .

Melica.

uniflora, 136.

nutans, 137. Allimia

Sesleria.

cœerulea, 139.

Glyceria.

aquatica, 140.

fluitans, 141 .

distans, 142.

maritima, 143.

procuinbens, 144.

Poa.

rigida, 145 .

compressa, 146 .

alpina, 147.

laxa, 148 .

bulbosa, 149 .

trivialis, 150.

pratensis, 151.

annua, 152.

glauea, 153.

nemoralis, 154.

Triodia.

decumbens, 355.

Briza.

minor, 156.

media, 157.

Dactylis.

glomerata, 158.

Spartina. 
stricta, 159.

Cynosurus.

echinatus, 160 .

cristatus, 161.

Festuca.

ovina, 162.

vivipara, 163.

duriuscula, 164.

rubra, 165.

bromoides, 166. z Nyg a luerdes

Myurus, $167 .=$

uniglumis, 168 . =

gigantea, 169. : Bromes

calamaria, 170 .

loliacea, 171 .

pratensis, 172.

elatior, 173.

sylvatica, 174.

pinnata, 175 .

Bromus.

secalinus, 176.

velutinus, 177 .

mollis, 178.

racemosus, 179.

squarrosus, 180.

arvensis, 181 .

erectus, 182.

asper, 183.

sterilis, 184.

Stipa.

diandrus, 185.

Aven:.

pennata, 186.

- fatua, 187.

strigosa, 188. : Xacthome pubescens, $189 .=$ Tre se tum pratensis, 190.

alpina, 191 .

flavescens, 192.

Lagurus.

ovatus, 193.

Arundo.

Phragmites, 194.

epigejos, 195.

calamagrostis, 196.

stricta, 197.

arenaria, 198.

Lolium.

perenne, 199.

temulentum, 200.

arvense, 201.

Rottbollia.

incurvata, 202.

Elymus.

arenarius, 203.

geniculatus, 204.

europæus, 205.

Hordeum.

murinum, 206.

pratense, 207.

maritinum, 208.

Triticum.

junceum, 209. repens, 210 .

caninum, 211.

cristatum, 212.

loliaceum, 213.

Trigria.

Montia.

foritaria, 214.

Holosteum.

umbellatum, 215.

Polycarpon.

tetraphyllum, 216.

TETRANDRIA.

Monogyni.

Dipsacus.

fullonum, $21 \%$.

sylvestris, 218 . pilosus, 219 .

Scabiosa.

succisa, 220 .

arvensis, 221.

columbaria, 222.

Sherardia.

arvensis, 223.

Asperula.

odorata, 224.

cynanchica, 225 .

Galium.

eruciatum, 226.

palustre, 227.

Witheringii, 228.

saxatile, $2 \cdot 29$.

uliginosum , 230 .

erectum, 231.

cinereum, 232.

aristatum, 233.

verrucosum, 234 .

tricorne, 235.

spurium, 236.

pusillum, 237.

verum, 238.

Mollugo, 239.

anglicum, 240.

boreale, 241 .

Rubia.

Aparine, 242.

peregrina, 243.

Exacum.

filiforme, 244 .

Plantago.

major, 245.

media, 246.

lanceolata, 247.

maritima, 248.

coronopus, 249.

Centunculus.

minimus, 250.

Sanguisorba.

officinalis, 251.

Epimedium. alpinum, 252.

Cornus.

sanguinea, 253.

Suecica, 254 . 
Parietaria.

officinalis, 255.

Isnardia.

palustris, 256.

Alchemilla.

vulgaris, 257.

alpira, 258.

arvensis, 259.

Digynia.

Buffonia.

tenuifolia, 260.'

TETRagYNia.

Ilex.

Aquifolium, 261.

Potamogeton.

natans, 262.

heterophyllum, 263.

perfoliatum, 264.

densum, 265.

fluitans, 266.

lucens, 267.

lanceolatum, 268.

crispum, 269.

compressum, 270.

cuspidatum, 271.

gramineum, 272.

pusillum, 273.

Ruppia.

pectinatum, 274.

Sagina.

maritima, 275.

procumbens, 276 .

maritima, 277.

apetella, 278 .

Mœnchia.

erecta, 279.

Tillæa.

muscosa, 280.

Radiola.

millegrana, 281.

PENTANDRIA.

MONOGYNIA.

Myosotis.

palustris, 282.

eæspitosa, 283.

intermedia, 284.

sylvatica, 285 .

alpestris, 286.

arvensis, 287.

versicolor, 288 .

Lithospermum.

officinale, 289 .

arvense, 290

purpuro-cœruleum, $29 \mathrm{l}$.

Anchusa. maritimuin, 292.

officinalis, 293.

sempervirens, 294.

Cynoglossum.

officinale, 295.

sylvaticum, 296.

Pulmonaria.

officinalis, 297.

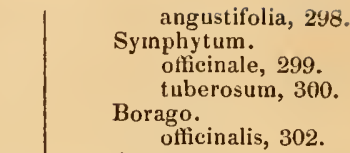

Asperugo.

procumbens, 301 .

Lycopsis.

arvensis, 303.

Echium.

- vulgare, 304.

Primula.

vulgaris, 305 .

elatior, 306 .

veris, 307 .

farinosa, 308 . scotica, 309.

Cyclamen.

hederifolium, 310.

Menyanthes.

trifoliata, 313.

Hottonia.

nymphrooides, 312.

palustris, 313.

Lysimachia.

vulgaris, 314.

thyrsiflora, 315 .

nemorum, 316.

Nummularia, 317.

Anagalis.

arvensis, 318.

ccerulea, 319.

tenella, 320 .

Azalea.

- prồcumbens, 321 .

Convolvulus.

arvensis, 322.

sepium, 323 .

Soldanella, 324.

Polemonium.

coruleum, 325.

Campanula. rotundifolia, 326 .

patula, 327 .

Rapuneulus, 328.

persicifolia, 329 .

latifolia, 330.

rapinculoides, 331 .

Trachelium, 332.

glomerata, 333.

hybrida, 334 .

hederacea, 335 .

Phytenma

orbiculare, 336 .

Jasione.

montana, 337.

Lobelia.

Dorturanna, 338.

urens, 339 ,

Impatiens.

Viola. 
hirta, $3 \pm 1$.

odorata, 342 .

palustris, $3+3$.

canina, 344 .

lactea, 345 .

flavicornis, 346 .

tricolor, 347.

lutea, $3 \pm 8$.

Verbascum.

Thapsus, 349.

Lychnitis, 350.

pulverulentum, 351 .

nigrum, 352.

virgatum, 353.

Blattaria, 354 .

\section{Datura.}

Stramonium, 355.

Hyoscyamus.

niger, 356.

Atropa.

Belladonna, 357.

Solanum.

Dulcamara, 358. nigrum, 359 .

Erythræa.

Centaurium, 360.

littoralis, 361 .

latifolia, 362.

pulchella, 363 .

Samolus.

Valerandi, 364.

Lonicera.

Caprifolium, 365.

Periclymenum, 366.

Xylosteum, 367.

Rhamnus.

catharticus, 368.

Euonymus.

Frangula, 369.

Ribes. europæus, 370.

rubrum, 371.

petræum, 372 .

spicatum, 373 .

alpinum, 374 .

nigrum, 375.

Grossularia, 376.

Hedera.

Helix, 377.

Illecebrum.

Glaux. verticillatum, 378.

maritima, 379 .

Thesium.

Vinca.

linophyllum, 380 .

minor, 381 . major, 382.

Digynia.

Herniaria.

glabra, 383 .

hirsuta, 384 .

Chenepodium.
Bonus Henricus, 385.

urbicum, 386 .

rubrum, 387.

botryodes, 388 .

murale, 389 .

hybridum, 390.

album, 391.

ficifolium, 392.

glaucum, 393.

olidum, 394.

polyspermum, 395.

acutifolium, 396 .

Beta.

maritimum, 397.

Salsola.

Kali, 399.

Ulmus.

fruticosa, 400 .

campestris, 401 .

suberosa, 402.

major, 403.

montana, 404 .

glabra, 405 .

Cuscuta.

europæa, 406.

Swertia.

Epithymum, 407.

perennis, 408 .

Gentiana.

Pneunionanthe, 409.

acaulis, 410 .

verna, 411 .

nivalis, 412 .

Amarella, 413.

campestris, 414 .

Eryngium.

maritimum, 415.

Sanicula. campestre, 416.

europæea, 417.

Echinophora.

spinosa, 418.

Daucus.

carota, 419.

maritimus, 420 .

Caucalis.

daucoides, 421.

latifolia, 422 .

Torilis.

Anthriseus, 423 .

infesta, 424 .

nodosa, 425 .

Anthriscus.

vulgaris, 426.

Scandix.

Pecten-Veneris, 427.

Chærophyllum.

sativum, 428.

sylvestre, 429 .

Myrrhis.

odorata, 430 .

temulenta, 43 ?. 
aurea, 432 .

aromatica, 433.

Bunium.

Sium.

flexuosum, 434.

latifolium, 435.

angustifolium, 436 .

nodiflorum, 437.

repens, 438 .

inundatum, 439 .

Sison. verticillatum, 440 .

Amomum, 441.

Cicuta. segetum, 442 .

Ethusa. virosa, 443

Conium. cynapium, 444. maculatum, 445.

Coriandrum. sativum, 446.

Enanthe.

fistulosa, 447.

pimpinelloides, 448. peucedanifolia, 449 . crocata, 450. Phellandrium, 451.

Crithmum. maritimum, 452.

Smyrnium.

Olusatrum, 453.

Apium.

graveolens, 454 .

Egopodium.

Podagraria, 455.

Imperatoria.

Ostruthium, 456.

Angelica.

Archangelica, 457. sylvestris, 458 .

Ligusticum.

scoticum, 459.

Meum. cornubierıse, 460 .

athamanticum, 461 .

Carum. Foniculum, 462

Carui, 463.

Athamanta.

Libanotis, 464.

Pimpinella.

saxifraga, 465 .

magna, 466.

dioica, 467.

Cnidium.

Silaus, 468.

Bupleurum. rotundifolium, 469 . Odontites, 470. tenuissimum, 471 .

Hydrocotyle.

vulgaris, 472 .
Selinum.

palustre, 473.

Peucedanum. officinale, 474 .

Pastinaca. sativa, 475 .

Heracleum. Sphondylium, 476 .

Tordylium. officinale, 477. maximum, 478. TRIgYNIA.

Viburnum.

Lantana, 479. Opulus, 480.

Sambucus.

Ebulus, 481. nigra, 482 .

Staphylea. pinnata, 483.

Tamarix. gallica, 484.

Corrigiola. littoralis, 485.

Tetragynia.

Parnassia. palustris, 486. Pentagrinia.

Statice.

Armeria, 487.

Limonium, 488.

Linum. reticulata, 489 .

usitatissimum, 490 . perenne, 491. angustifolium, 492. catharticum, 493.

Sibbaldia. procumbens, 494. HEXAGYNIA.

Drosera. rotundifolia, 495 . longifolia, 496. anglica, 497.

Polygynia.

Myosurus. minimus, 498.

HEXANDRIA.

Monogrnja.

Galanthus. nivalis, 499 .

Leucojum. æestivum, 500.

Narcissus. poeticus, 501 . biflorus, 502 .

Allium. Pseudo-narcissus, 503.

Ampeloprasum, 504. arenarium, 505. carinatum, 506. oleraceum, 507. vineale, 508 . 
ursinum, 509. Fritillaria.

Schœnoprasum, 510 .

Tulipa.

Meleagris, 511.

Sylvestris, 512.

Ornithogalum.

luteum, 513.'

pyrenaicum, 514.

umbellatum, 515 .

Scilla.

nutans, 516.

verna, 517.

bifolia, 518 .

autumnalis, 519 .

nutans, 520.

Hyacinthus. racemosus, 521 .

Anthericum. serotinum, 522 .

Narthecium. ossifragum, 523.

Asparagus. officinalis, 524 .

Convallaria.

majalis, 525 .

verticillata, 526 .

Polygonatum, 527. multiflora, 528 .

Acorus.

Calamus, 529.

Juncus.

acutus, 530.

maritimus, 531 .

glaucus, 532 .

conglomeratus, 533 .

effusus, 534.

filiformis, 535 .

areticus, 536 .

trifidus, 537.

squarrosus, 538.

compressus, 539.

conosus, 540.

Gesneri, 541.

bufonius, 542 .

uliginosus, 543 .

capitatus, 544 .

biglumis, 545.

triglumis, 546 .

castaneus, 547.

acutiflorus, 548 .

lampocarpus, 549.

obtusiflorus, 550 .

polycephalus, 551 .

Luciola.

pilosa, 552 .

Forsteri, 553.

sylvatica, 554 .

eampestris, 555.

congesta, 556 .

spicata, 557.

arcuata, 558.

Berberis. vulgaris, 559 .

Frankenia.

læevis, 560.

pulverulenta, 561.

Peplis.

Portula, 562.

DigYNiA.

Oxyria.

reniformis, 563 .

Trigynia.

Rumex.

sanguineus, 564 .

crispus, 565.

acutus, 566.

obtusifolius, 567 .

pulcher, 568.

maritimus, 569.

palustris, 570 .

Hydrolapathum, 571 .

Acetosa, 572.

Acetosella, 573.

Scheuchzeria. palustris, 574 .

Tofieldia. palustris, 575 .

Triglochin.

palustre, 576.

maritimum, 577.

Colchicum, autumnale, 578 .

Polygynia.

Alisma.

Plantago, 579.

Dainasonium, 580 .

natans, 581 .

ranunculoides, 582.

repens, 583.

HEPTANDRIA.

Monogynia.

Trientalis.

europæa, 584.

OCTANDRIA.

Monogynia.

Enothera.

biennis, 585 .

Epilobium.

angustifolium, 586 .

hirsutum, 587.

parviflorum, 588.

montanum, 589 .

roseum, 590 .

tetragonum, 591 .

palustre, 592.

alsinifolium, 593 .

alpinum, 594.

Chlora.

perfoliata, 595.

Vaccinium.

myrtillus, 596.

uliginosum, 697.

Vitis Idæa, 598.

oxycoccus, 599.

Menziesia. 
ccrulea, 600.

Calluna. olifolia, 601 .

\section{Erica.}

vulgaris, 602.

Tetralix, 603. cinerea, 604. vagans, 605 . ciliaris, 606 .

\section{Daphne.}

Mezereum, 607.

Acer. Laureola, 608.

Pseudo-platanus, 609. campestre, 610.

Trigynia.

Polygonum. amphibium, 611.

Persicaria, 612. lapathifolium, 613 . Hydropiper, 614. minus, 615 .

Bistorta, 616.

viviparum, 617 .

aviculare, 618 .

Fagopyrum, 619. convolvulus, 620 .

Tetragynia.

Paris.

Adoxa.

quadrifolia, 621 .

Elatine.

Moschatellina, 622 .

tripetala, 623 .

ENNEANDRIA.

HEXAGTNIA.

Butomus.

umbellatus, 624 .

DECANDRIA.

Monogynia.

Monotropa.

Hypopitys, 625.

Andromeda. polifolia, 626 .

Arbutus.

unedo, 627.

alpina, 628 .

Pyrola. uva ursi, 629 .

rotundifolia, 630 .

media, 631 .

minor, 632 .

secunda, 633 .

uniffora, 634.

DigYNiA.

Chrysosplenium.

alternifolium, 635 .

oppositifolium, 636 .

Saxifraga.

Geum, 637.

hirsuta, 638 .

umbrosa, 639 .

stellaris, 640 . nivalis, 641 .

oppositifolia, 642 .

Hirculus, 643.

aizoides, $6 \pm 4$.

granulata, $6+5$.

cernua, 646 .

rivularis, $6 \pm 7$.

tridactylites, 648 .

muscoides, 649 .

pygmæa, 650.

caspitosa, 651 .

hirta, 652.

platypetala, 653 .

hypnoicles, 654 .

elongella, 655 . pedatifida, 656 .

Scleranthus.

annuus, 657.

perennis, 658 .

Saponaria.

officinalis, 659 .

Dianthus.

Armeria, 660.

prolifer, 661 .

caryophyllus, 662.

deltoides, 663 .

cæsius, 664.

TRGYNIA.

Silene.

anglica, 665 .

quinquevulnera, 666 .

inflata, 667 .

maritima, 668.

conica, 669.

noctiflora, 670 .

armeria, 671 .

nutans, 672 .

Otites, 673.

acaulis, 674 .

Stellaria.

nemorum, 675 .

media, 676 .

holostea, 677 .

graminea, 678.

glauca, 679 .

uliginosa, $6 \mathrm{~S} 0$.

scapigera, 681.

cerastoides, 682 .

Arenaria.

peploides, 683.

trinervis, 684.

serpyllifolia, 685

tenuifolia, 686 .

verna, 687.

rubella, 688 .

fastigiata, 689 .

ciliata, 690 .

rubia, 691 .

marina, 692.

Cherleria.

sedoides, 693.

PENTAGYNIA.

Cotyledon. 
Umbilicus, 694. lutea, 695 .

Sedum.

Telephium, 696.

dasyphyllum, 697.

anglicum, 698 .

acre, 699.

sexangulare, 700 .

villosum, 701 .

album, 702.

reflexum, 703 .

glaucum, 704 .

rupestre, $7 \mathrm{t} 5$.

Oxalis.

Forsterianum, 706.

Acetosella, 707 . corniculata, 708 .

Agrostemma.

Lychnis.

Githago, 709.

Floscuculi, 710 .

viscaria, 711 .

alpina, 712 .

dioica, 713 .

Cerastium.

vulgatum, 714 .

viscosum, 715 .

semidecandrum, 716 .

tetrandrum, 717.

arvense, 718 .

alpinum, 719 .

latifolium, 720 .

aquaticum, 721 .

Spergula.

arvensis, 722 .

nodosa, 723 .

saginoides, 724 .

subulata, 725 .

DODECANDRIA.

MONOGYNIA.

Asarum.

europæum, 726.

Lythrum.

Salicaria, 727.

hyssopifolium, 728.

DIGYNIA.

Agrimonia.

Eupatoria, 729.

TRIGYNIA.

Reseda.

Luteola, 730 .

lutea, 731.

DODECAGYNIA.

Sempervivum. tectorum, 732 .

ICOSANDRIA.

MONOGYNIA.

Prunus.

Padus, 732.

Cerasus, 734.

domestica, 735 .

insititia, 736 .

spinosa, 737 .
PENTAGYNIA.

Mespilus.

Oxyacantha, 738.

germanica, 739 .

Cotoneaster, 740 .

Pyrus.

communis, 741 .

malus, 742 .

torminalis, 743 .

domestica, 744 .

auccuparia, 745 .

pinnatificla, 746 .

Aria, 747.

Spiræa.

salicifolia, 748 .

Filipendula, 749 .

ulmaria, 750 .

POLYGYNIA.

Rosa.

cinnamomea, 75].

rubella, 752.

spinosissima, 753 .

involuta, 754.

Doniana, 755 .

gracilis, 756 .

Sabini, 757.

villosa, 758 .

tomentosa, 759 .

rubiginosa, 760 .

micrantha, 761 .

Borreri, 762.

cæsia, 763 .

sarmentacea, 764 .

dumetorum, 765 .

Forsteri, 766.

hibernica, 767.

canina, 768 .

systyla, 769 .

arvensis, 770 .

Rubus.

fruticosus, 771 .

plicatus, 772.

rhamnifolius. 773 .

lencostachys, 774 .

suberectus, 775 .

idæus, 776 .

corylifolius, 777 .

cæesius, 778 .

saxatilis, 779 .

areticus, 780 .

Chamæmorus, 781.

Fragaria.

vesca, 782.

elatior, 783 .

Potentilla.

fruticosa, 784 .

anserina, 785 .

rupestris, 786 .

argentea, 787.

alpestris, 788 .

verna, 789.

opaca, 790.

alba, 791 . 
reptans, 792.

tridentata, 793.

Fragariastrum, 794.

Tormentilla.

officinalis, 795 .

reptans, 796 .

Geum.

urbalum, 797.

rivale, 798 .

Dryas.

octopetala, 799.

Comarum.

palustre, 800 . POLYANDRIA.

MONOGYNIA.

Actra.

spicata, 801.

Chelidonium. majus, 802.

Glaucium. luteum, 803. phœniceum, 804. violaceum, 805 .

Papaver.

hybridum, 806 .

Argemone, 807.

Dubium, 808.

Rhœas, 809.

somniferum, 810 .

cambricum, 811.

Nymphæa.

Nuphar. alba, 812 .

Tilia.

lutea, 813.

pumila, 814 .

europæa, 815.

grandifolia, 816 . parvifolia, 817 .

Cistus.

marifolius, 818 .

guttatus, 819 .

ledifolius, 820 .

surrejanus, 821 .

Helianthemum, 822.

tomentosus, 823 .

polifolius, 824 .

Pentagynia.

Pœonia.

corallina, 825 .

Delphinium.

Aconitum.

consolida, 826.

Napellus, 827.

Aquilegia.

vulgaris, 828 .

Stratiotes.

aloides, 829.

Polygynia.

Anemone.

Pulsatilla, 830.

nemorosa, 831.

apennina, 832 . ranunculoides, 833 .

Clematis. vitalba, 834 .

Thalictrum.

alpinum, 835.

minus, 836.

majus, 837.

flavum, 838 .

Adonis. autumnalis, 839 .

Ranunculus.

Flammula, 840.

Lingua, 841.

gramineus, 842.

Ficaria, 843.

auricomus, 844 .

sceleratus, 845 .

alpestris, 846 .

bulbosus, 847 .

hirsutus, 848 .

repens, 849 .

acris, 850 .

arvensis, 851 .

parviflorus, 852 .

hederaceus, 853.

aquatilis, 854 .

Trollius.

$$
\text { europæus, } 855
$$

Helleborus.

viridis, 856 .

Caltha. foetidus, 857 .

palustris, 858 . radicans, 859 .

DIDYNAMIA.

GYMNOSPERMIA.

Ajuga.

reptans, 860 .

alpina, 861 .

Pyramidalis, 862.

Teucrium.

Chamœepitys, 863.

Scorodonia, 864.

Scordium, 865.

Nepeta.

Chamædrys, 866.

cataria, 867.

Verbena.

officinalis, 868.

Mentha.

sylvestris, 869 .

rotundifolia, 870 .

viridis, 871 .

piperita, 872 .

citrata, 873.

hirsuta, 874 .

acutifolia, 875 .

rubra, 876 .

gentilis, 877 .

gracilis, 878 .

arvensis, 879 .

agrestis, 880 .

Pulegium, 881. 
Glechoma.

hederacea, 882 .

\section{Lamium.}

album, 883.

maculatum, 884 .

purpureum, 885.

incisum, 886.

amplexicaule, 887 .

Galeopsis.

Ladanum. 888.

villosa, 889 .

Tetrahit, 890 .

versicolor, 891 .

Galeobdolon.

luteum, 892.

Betonica.

officinalis, 893.

Stachys.

sylvatica, 894 .

ambigua, 895.

palustris, 896 .

germanica, 897.

Ballota.

arvensis, 898.

nigra, 899.

Marrubium.

rulgare, 900.

Leonurus.

cardiaca, 901 .

Clinopodium.

vulgare, 902.

Origanum. vulgare, 903.

Thymus.

Serpyllum, 904.

Acinos, 905.

Calamintha. 906.

Melittis.

Nepeta, 907.

Melisophyllum, 908. grandiflora, 909.

Scutellaria. galericulata, 910. minor, 911.

Prunella.

vulgaris, 912.

ANGIOSPERMIA.

Bartsia.

Alpina, 913.

viscosa, 9]4.

Odontites, 915.

Rhinanthus.

Crista galli, 916.

major, 917.

Euphrasia.

officinalis, 918.

Melampyrum.

cristatum, 919.

arvense, 920.

pratense, 921 .

sylvaticum, 922 .

Lathræe.

squamarea, 923.
Pedicularis.

palustris, 924 .

sylvatica, 925 .

Antirrhinum.

Cymbalaria, 926.

spurium, 927.

Elatine, 928.

repens, 929 .

linaria, 930.

minus, 931.

majus, 932.

Orontium, 933.

Serophularia.

nodosa, 934.

aquatica, 935.

Scorodonia, 936 .

Digitalis.

vernalis, 937 .

Linnæa. purpurea, 938.

borealis, 939 .

Sibthorpia. europæa, 940.

Limosella. aquatica, 941.

Orobanche.

major, 942.

elatior, 943.

minor, 944.

rubia, 945 .

corrulea, 946 .

ramosa, 947 .

TETRADYNAMIA.

Siliculosa.

Vella.

annua, 948.

Subularia.

Draba.

aquatica, 949.

verna, 950.

aizoides, 951 .

hirta, 952.

incana, 953.

muralis, 954 .

Alyssum.

maritimum, 955.

Camelina. sativa, 956.

Lepidium. latifolium, 957. ruderale, 958 . campestre, 959. hirtum, 960 .

Hutchinsia. petræa, 961 .

Teesdalia. nudicaulis, 962 .

Thlaspi.

$$
\text { arvense, } 963 .
$$

perfoliatum, 964.

alpestre, 965 .

Bursa Pastoris, 966.

Cochlearia. 
officinalis, 967 .

grœulandica, 968 . anglica, 969 . danica, 970.

Armoracia, 971.

Senebiera.

Coronopus, 972.

Iberis. didyma, 973 .

1satis. amara, 974 .

Cakile. tinctoria, 975 .

maritima, 976.

Crambe. maritima, 977.

Siliquosa.

Dentaria. bulbifera. 978 .

Cardamine. bellilifolia, 979 . impatiens, 980. hirsuta, 981. pratensis, 982. amara, 983.

Nasturtium.

officinale, 984. sylvestre, 985 . terrestre, 986 . amphibium, 987.

Sisymbrium. officinale, 988. Trio, 989. Sophia, 990.

Barbarea. vulgaris, 991. præecox, 992.

Erysimum. cheiranthoides, 993 .

Alliaria, 994. orientale, 995 .

Cheiranthus. fruticulosus, 996 .

Matthiola.

incana, 997. sinuata, 998.

Hesperis. matronalis, 999 .

Arabis.

thaliana, 1000. stricta, $100 \mathrm{I}$. hispida, 1002. ciliata, 1003. hirsuta, 1004.

Turrita. turrita, 1005.

glabra, 1006.

Brassica.

napus, 1007. Rapa, 1008. campestris, 1009. oleracea, 1010. monensis, 1011.
Sinapis.

arvensis, 1012

alba, 1013

nigra, 1014

tenuifolia, 1015 .

muralis, 1016.

Raphanus.

Raphanistrum, 1017.

maritimus, 1018.

MONADELPHIA.

Pentandria.

Erodium.

cicutarium, 1019.

moschatum, 1020.

maritimum, 1021.

DECANDRIA.

Geranium.

phæum, 1022.

nodosum, 1023.

sylvaticum, 1024 .

pratense, 1025 .

robertianum, 1026.

lucidum, 1027.

molle, 1028.

pusillum, 1029.

pyrenaicum, 1030 .

rotundifolium, 1031 .

dissectum, 1032.

columbinum, 1033.

sanguineum, 1034.

Polyandria.

Althæa.

officinalis, 1035.

Malva.

sylvestris, 1036.

rotundifolia, 1037.

moschata, 1038.

Lavatera.

arborea, 1039.

DIADELPHIA.

HEXANDRIa.

Fumaria.

solida, 1040.

lutea, 1041.

claviculata, 1042.

officinalis, 1043.

parviflora, 1044. capreolata, 1045.

OctandRIa.

Polygala. vulgaris, 1046.

DECANDRIa.

Spartium.

Genista. scoparium, 1047.

tinctoria, 1048.

pilosa, 1049 .

Ulex. anglica, 1050.

europæus, 1051. nanus, 1052.

Ononis.

spinosa, 1053. 
Anthyllis.

vulneraria, 1054.

Pisum.

maritimum, 1055.

Orobus

tuberosus, 1056.

ni =er, 1057.

sylvaticus, 1058.

Lathyrus.
Alphaca, 1059.
Nissolia, 1060.
hirsutus, 1061 .
pratensis, 1062.
s Ivestris, 1063.
latifolius, 1064.
palustris, 1065.

Vicia.

sylvatica, 1066.

Cracea, 1067.

sativa, 1068.

angustifolia, 1069 .

lathyroides, 1070 .

lutea, 1071 .

hybrida, 1072.

laevigata, 1073.

sepium, 1074.

Ervum.

bithynica, 1075 .

tetraspermum, 1076.

hirsutum, 1077.

Ornithopus. perpusillus, 1078.

Hippocrepis. comosa, 1079.

Hedysarum.

Onobrychis, 1080.

Astragalus.

glycyphyllos, 1081.

hypoglottis, 1082 .

uralensis, 1083.

campestris, 1084.

Trifolium.

officinale, 1085.

ornithopodiodes, 1086.

repens, 1087.

suffoeatun, 1088

subterraneum, 1089.

ochroleucum, 1090.

pratense, 1091.

medium, 1092.

maritimum, 1093.

stellatum, 1094.

arvense, 1095.

scabrum, 1096.

glomeratum, 1097.

striatum, 1098.

fragiferum, 1099 .

procumberis, 1100.

minus, 1101 .

filiforme, 1102.

Lotus.

corniculatus, 1103.

major, 1104. decumbens, 1105.

angustissimus, 1106.

Medicago.

sativa, 1107.

falcata, 1108

lupulina, 1109.

maculata, 1110.

muricata, 1111.

minima, 1112.

POLYADELPHIA.

YOLYANDRIA.

Hypericum

calycinum, 1113.

Androsæmum, 1114.

quadranyulum, 1115 .

perforatum, 1116.

dubium, 1117.

humifusum, 1118.

molitarium, 1119.

barbatum, 1120.

hirsutum, 1121.

pulchrum, 1122.

elodes, 1123.

SYNGENESIA.

POLYGAMA EQUALIS.

Tragopogon.

pratensis, 1124.

Pieris.

parvifolius, 1125.

echioides, 1126.

hieracioides, 1127.

Sonchus.

coeruleus, 1128.

palustris, 1129.

arvensis, 1130.

oleracens, 1131 .

Lactuca.

virosa, 1132.

Scariola, 1133.

saligna, 1134.

Prenanthes.

muralis, 1135.

Leontodon.

Taraxacum, 1136. palustre, 1137.

Apargia.

hispida, 1138.

hirta, 1139.

Taraxaci, 1140.

autumnalis, 1141 .

Hieracium.

alpinum, 1142.

Pilosella, 1143.

dubium, 1144.

Auricula, 1145.

aurantiacum, 1146.

murorum, 1147.

maculatum, 1148.

sylvaticum, 1149 .

pulmonarium, 1150.

Lawsoni, 1151.

puludosum, 1152.

molle, 1153. 
cerinthoides, 1154 . villosum, 1155 . Halleri, 1156. subandum, 1157. denticulatum, 1158 . prenanthoides, 1159. umbellatum, 1160 .

Crepis.

foetida, 1161.

pulchra, 1162.

tectorum, 1163.

biennis, 1164.

Hypocharis.

maculata, 1165.

glabra, 1166.

Lapsana. radicata, 1167.

communis, 1168. pusilla, 1169.

Cichorium.

Aretium Intyhus, 1170.

Lappa, 1171.

Barlana, 1172.

Serratula.

tinctoria, 1173.

Carduus. alpina, 1174 .

nutans, 1175. acanthoides, 1176. tenuiflorus, 1177 .

Cnicus. marianus, 1178.

lanceolatus, 1179.

palustris, 1]80. arvensis, 1181. eriophorus, 1182. tuberosus. 1183.

heterophyllus, 1184. praterisis, 1185. acaulis, 1186 .

Onopordum. Acanthium, 1187.

Carlina.

Bidens. vulgaris, 1188.

tripartita, 1189. cernua, 1190.

Eupatorium. cannabinum, 1191.

Chrysocoma.

Diotis. Linosyris, 1192.

maritima, 1193.

POLYGAIIA SUPERFLU.

Tanacetum. vulgare, 1194.

Artemisia. camnestris, 1195. maritima, 1196. gallica, 1197.

Absinthium, 1198. vulgaris, 1199. coerulescens, 1200.

Gnaphalium.

luteo-album, 1201.

margaritaceum, 1202.

dioicum, 1203.

sylvaticum, 1204.

rectum, 1205.

supinum, 1206.

uliginosum, 1207.

gallicum, 1208 .

minimum, 1209.

germanicum, 1210.

Conyza.

squariosa, 1211.

Erigeron.

canadensis, 1212.

acris, 1213 .

alpinus, 1214.

unifiorus, 1215.

Tussilago.

Faifara, 1216.

Petasites, 1217.

Senecio.

vulgaris, 1218.

viscosus, $] 219$.

lividus, 1220 .

sylvaticus, 1221.

squalidus, 122.2.

tenuifolius. 1223.

Jacobra, 122-1.

aquaticus, 1225.

paiudosus, 1226.

saracenicus, 1227.

Aster.

Tripolium, 1228.

Solidago.

Inula. virgaurea. 1229.

Hclenium, 1230. dysenterica, 1231 . pulicaria, 1232.

Cineraria.

crithmoides, 1233.

palustris, 1234.

integrifolia, 123.5.

Doronicum.

Bellis. Pardalianclıes, 1236.

perennis, 1237.

Chrysanthemum.

Leucanthemum, 1238.

segetum, 1239.

Pyrethrum.

Partlenium, 1240.

inodorum, 1241.

maritimum, 1242.

Matricaria. chamoinilla, 1243.

Anthemis.

maritima, 1244.

nobilis, 1245 .

arvensis, 1246.

Cotula, 1247. 
tinctoria, 1248 .

Achillea.

Ptarmica, 1249.

serrata, 1250.

Millefolium, 1251 .

tomentosa, 1252 .

Polygamia Frustranea.

Centaurea.

Jacea, 1253.

nig ra, 1254.

Cyanus, 1255.

Scabiosa, 1256.

Isnardi, 1257 .

Calcitrapa, 1258.

solstitialis, 1259.

G YNANDRIA.

Monandria.

Orehis.

bifolia, 1260.

pyramidalis, 126].

Norio, 1262 .

mascula, 1263.

ustulata, 1264.

fusca, 1265 .

militaris, 1266 .

tephrosanthus, 1267.

hircina, 1268.

albida, 1269.

viriclis, 1270.

latifolia, 1271.

maculata, 1272 .

conopsea, 1273.

Aceras.

anthropophora, 1274.

Herminium.

monorchis, 1275.

Ophrys.

muscifera, 1276.

apifera, 1277 .

arachnites, 1278.

aranifera, 1279 .

fuciîera, 1280 .

Goodyera.

repens, 1281 .

Neottia.

spuralis, 1282. gemmipara, 1283.

Listera.

ovata, 1284.

cordata, 1285.

Nidus Avis, 1286.

Epipactis.

latifolia, 1.287.

purpurata, 1288.

palustris, 1289.

grandiflora, 1290.

ensifolia, 1291.

rubra, 1292.

Malaxis.

paludosa, 1293.

Loeselii; 1294.

Corallorrhiza.

innata, 1295.
Diaxinia.

Cypripedium.

Calceolus, 1296.

HEXaninia.

Aristoloclia.

Clematitis, 1297.

MONOECIA.

Monanorla.

isuphorbia.

peplis, 1298.

peplus, 1299 .

exigua, 1300 .

Lathyris, $130 \mathrm{~J}$.

portlandica, 1:302.

paralia, 1303.

helioscopıa, 1304 .

stricta, 1305.

Esula, 1306

Cyparissias, 1307.

hiberna, 1308.

amygdaloides, 1309.

Characias, 1310.

Zanrichellia.

palustris, 1311.

Triavoria.

Typha.

latifolia, 1312.

angustifolia, 1313.

minor, 1314.

Sparganium.

ramosum, 1315.

simplex, 1316.

natans, 1317.

Carex.

dioica, 1318.

Davalliana, 1319.

pulicaris, $13: 0$

pauciflora, 1321.

stellulata, 1322 .

curta, 1323.

elongata, 1324.

ovalis, 1325.

remota, 1326 .

axillaris, 1327.

incurva, ]328.

alenaria, 1329.

intermedia, 1330 .

divisa, $133 !$.

muricata, 133.2.

divulsa, 1333 .

vulpina, 1334 .

teretiuscula, 1335.

paniculata, 1836 .

digitata, 1337.

clandestina, 1338.

pendula, 1339.

strigosa, 1340.

sylvatica, 134].

depauperata, 1342.

Mlielichoferi, 1343.

speirostachya, 1344 .

phæoostachya, 134j.

capillaris, 1346. 
rariflora, 1347.

Pseudo-cyperus, 1348.

limosa, 1349.

ustulata, 1350.

atrata, 1351 .

pulla, 1352.

pallescens, 1353.

flava, 1354.

Eederi, 1355.

fulva, 1356.

extensa, 1357.

distans, 1358.

binervis, 1359 .

pracox, 1360.

pilulifera, 1361 .

panicea, 1362.

recurva, 1363.

rigida, 1364 .

caespitosa, 1365.

stricta, 1366.

acuta, 1367.

paludosa, 1368.

riparia, 1369.

lovigata, 1370.

vesicaria, 1371 .

ampullacea, 1372 .

hirta, 1373.

stictocarpa, 1374.

angustifolia, 1375 .

filiformis, 1376.

Kobresia.

caricina, 1377.

TetrandRia .

Littorella.

Alnus.

lacustris, 1378 .

Buxus.

glutinosa, 1379.

Urtica. sempervirens, 1380 .

pilulifera, 1381.

urens, 1382.

dioica, 1383.

Pentandria.

Xanthium.

strumarium, 1384.

Amaranthus.

Blitum, 1385.

Bryonia.

dioica, 1386

HEXANDRIA.

Eriocaulon. septangulare, 1387.

Polyandria.

Ceratophyllum. demersum, 1388. submersum, 1389 .

Myriophyllum.

spicatuın, 1390.

verticillatum, 1391.

Sagittaria.

sagittifolia, 1392.

Arum. maculatum, 1393.

Poterium.

Sanguisorba, 1394.

Quercus.

Robur, 1395.

Fagus.

sessiliflora, 1396.

Castanea, 1397.

Betula.

Sylvatica, 1398.

alba, 1399.

nana, 1400.

Carpinus.

Corylus.

Betulus, 1401.

Pinus.

Avellana, 1402.

sylvestris, 1403.

DIOECIA.

I Salix.

Diandeia.

triandra, 1404.

Hoffmanniana, 1405.

lanceolata, 1406 .

amygdalina, 1407.

pentandra, 1408.

nigricans, 1409.

phylicifolia, 1410 .

Borreriana, 1411.

nitens, 1412.

Davalliana, 1413.

Wulfeniana, 1414.

tetrapla, 1415.

bicolor, 1416.

tenuifolia, 1417.

malifolia, 1418 .

petiolaris, 1419.

vitellina, 1420.

decipiens, 1421.

fragilis, 1422.

Russelliana, 1423.

purpurea, 1424.

Helix, 1425.

Lambertiana, 1426.

Forbialla, 1427.

rubra, 1428.

Cron eana, 1429

prunifolia, 1430.

vacciuiifolia, 1431.

venulosa, 1432.

myrsinites, 1433.

Dicksoniana, 1434.

carinata, 1435.

Arbuscula. 1436.

herbacea, 1437 .

reticulata, 1438 .

glauca, 1439.

Stuartiana, 1440.

arenaria, 1441 .

lanata, 1442.

argentea, 1443.

foetida, 1444.

repens, 1445. 
fusca, 1446.

prostrata, 1447.

incubacea, 1448.

Doniana, 1449.

rosmarinifolia, 1450 .

cinerea, 1451.

aurita, 1452.

aquatica, 1453.

oleifolia, 1454 .

cotinifolia, 1455.

hirta, 1456.

rupestris, 1457 .

Andersoniana, 1458.

Forsteriana, 1459.

sphacelata, 1460.

caprea, 146I.

acuminata, 1462.

viminalis, 1463.

Smithiana, 1464.

stipularis, 1465.

alba, 1466.

TriandRIA.

Empetrum. nigrum, 1467.

Ruscus. aculeatus, 1468 .

TETHANDRIA.

Viscum.

album, 1469.

Hippophaё. rhamnoides, 1470 .

Myrica.

Gale, 1471.

Pentandria.

Humulus.

Lupulus, 1472.

HEXANDRIA.

Tamus.

communis, 1473.

OctandRIA.

Populus.

alba, 1474

canescens, 1475.

tremula, 1476.

nigra, 1477 .

Rhodiola.

rosea, 1478.

ENNEANDRIa.

Mercurialis.

perennis, 1479 annua, 1480.

Hydrocharis.

Morsus Ranæ, 1481.

Monadelphia.

Juniperus.

communis, 1482.

nana, 1483.

Taxus.

baccata, 1484 .

POL YGAMIA.

Monoecia.

Atriplex.

portulacoides, 1485.

laciniata, 1486.

patula, 1487.

angustifolia, 1488.

erecta, 1489 .

littoralis, $1490 . \quad$ r 9 )

pedunculata. 1491. r hogo

Chara.

Hedwigii, 1492.

aspera, 1493.

Phyteuma.

Carex. spicatum, 1494.

tomentosa, 1495.

Petroselinum.

sativum, 1496.

Erica.

Mediterranea, 1497.

Elatine.

Hydropiper, 1498.

Cucubalis.

baccifer, 1499.

Anonis.

arvensis, 1500 .

Lepidium.

Draba, 1501.

Astragalus.

alpinus, 1502 .

1Trifolium.

2 resupinatum, 1503.

Antennaria.

Hyperborea, 1504.

Digitaria.

humifusa, 1505.

Rubus.

affinis, 1506. 


\section{ENGLISH INDEX}

TO THE MOST POPULAR GENERIC AND SPECIFIC NAMES.

Apple-tree, 742.

Agrimony, 729.

Alder, 1379.

Alexanders, 453.

Alkanet, 293.

All-seed, 216.

Andromeda, 626.

Arrow-grass, 576.

Arrow-head, 1392.

Asarabacea, 726.

Ash-tree, 16.

Asparagus, 524.

Aspen, 1476.

Avens, 799 .

Awlwort, 949.

-Azalea, 321.

Bane-berries, 801 .

Basil, 902,

Barley, meadow, 207.

_- , sea, 208. wall, 206.

Barrenwort, 252.

Bastard Balm, 908.

- Pimpernel, 250.

Toad-flax, 380 .

Beami-tree, 747.

Bear's-foot, 857.

- Beech, 1398.

Bed-straw, 226.

Belleisle-cress, 991.

Bell-flower, 326.

Bent-grass, 114.

Berberry, 559.

Berry-bearing Alder, 369.

Betorıy, 893.

Bilberry, 596 .

Bind-weed, 322.
Birch, 1399.

Bird's-eye, Primrose, 308.

Bird's-foot, 1078.

Bird's-foot, Trefoil, 1103.

Birth-wort, 1297.

Bistort, 616.

Black Bryony, 1473. Bind-weed, 620 .

Blackthorn, 737 .

Bladder-nut, 483.

Bladder-wort, 41.

Blue-bottle, 1255.

Bog-bean, 313.

Borage, 301.

Box-tree, 1380.

Bramble, 771 .

Brome-grass, 176.

Brook-lime, 26.

Brook-weed, 364.

Broom-rape, 942.

Broom, 1047.

Briony, 1386.

Buckbean, 313.

Buekthorn, 368.

Buck wheat, 619 .

Bugle, 860 .

Bugloss, 304.

Bullace, 736 .

Bullrush, 74.

Burdock, 1171.

Burmarygold, 1189.

Burnet, 251.

Salad, 1394.

Burnet saxifrage, 465 .

Burreed, 1315.

Burweed, 1384.

Buteher's-broom, 1468. 
Butter-bur, 1217.

Butter-cup, 847.

Butter-wort, 38.

Cabbage, 1010.

Calamint, 906.

Calathian-violet, 409 .

Canary-grass, 95.

Candy-tuft, 974.

Caraway, 463.

Carline-thistle, 1188.

Carnation, 662.

Carrot, 419.

Catehfly, 665.

Catmint, 867.

Cat's-ear, 1165.

Cat's-tail, 1312.

Cat's-tail Grass, 97.

Celandine, 802 .

Celery, 454 .

Chaff-weed, 250.

Chamomile, 1244 .

Charlock, 1012.

Cherry-wild, 734. Bird, 733.

Chervil, 428.

Chestnut, 1397.

Chickweed, common,676.

-

- $\longrightarrow$, sea, 683.

Chives. 510 .

Cinquefoil, 784.

Cleavers, 242.

Clover, common, I091.

-, Duteh, 1087.

Cloudberry, 781 .

Cock's-foot, 158.

Codlings and cream, 587.

Cole-seed, 1007.

Colt's-foot, 1216.

Columbine, 828.

Comfrey, 299.

Coral-root, 1295.

Coriander, 446.

Corn-coekle, 709.

Corn-marigold, 1239.

Corn-salad, 57.

Cornish-money wort, 940 .

Cotton-grass, 87.

Cotton-thistle, 1187.

Cotton-weed, 1193.

Couch-grass, 210.

Cow-berry 598.

Cow-parsnep, 476.

Cowslip, 307.

Cow -wheat, 919.

Crabtree, 742.

Crakeberry, 1467.

Cranberry, 599.

Crane's-bill, 1022.

Cress, 984.

Crow-berry, 1467.
Crowfoot, 840 .

Cuckoo-pint, 1393.

Cudweed, 1201.

Currant, 371 .

Datfodil, 503.

-, chequered,

Daisy, 1237.

Dame's violet, 999.

Dandelion, 1136.

Darnel, 199.

Deadly-night shade, 357 .

Dead-nettle red. 885.

Devil's-bit, 220.

Doek, 564.

Dodder, 406 .

Dog violet, 344 .

Dog-woorl, 253.

Don's-tail grass, 161.

Doy's-tooth grass, 119.

Drop-wort, 749 .

Duck-weed, 44.

Dutch myrtle, 1471.

Dwale, 357.

Dyer's Rocket, 730. Woad, 975.

Earth-nut, 434.

Elder, 481.

Elecampane, 1320.

Elm, 401.

- , giant, 403.

witch, 404

Enchanter's nightshade, 18.

English galingale, 69.

Evening primrose, 585.

Feather grass, 186 .

Fescue grass, 162.

1 haril, 164.

- tall, 169.

Fornel, 462.

Feverfew, 1240.

Figwort, 934.

Finger-grass, 120.

Flax, 490.

- seed, 281.

Flea-bane, 1212. wort, 1234.

Flowering rush, 624 .

Fly Honeysuckle, 367 .

Fool's-parsley, 444.

Fox-glove, 938.

French Willow, 586.

Fritillary, 511.

Frog-bit, 1481.

Fuller's Teasel, 217.

Fumitóry, 1040.

Furze, 1051.

Garden An:elica, 457.

Germander, $\$ 64$.

German Madwort, 302.

Gill, 882.

Gipsy-wort, 48 . 
Globe-flower, 855.

Goat's-beard, 1124.

Gold of Pleasure, 956.

Golden Rod, 1229.

_- Samphire, 1233.

Saxiliage, 635.

Goldilocks, 1192.

Givosebeiry, Common376

Goosefoot, 1385.

Gorse, 1051.

Gout-weed, 455.

Grass of Parnassus, 486.

Great Henhit, 887

_- Valerian, 55.

Gromwell, 289.

Grounul-ivy, 882.

Ground Pine, 683.

Groundse!, 1218.

Guelder Rose, 479.

Hare's-ear, 469.

Hare's-tail, 193.

Hawk -weed, 1142.

Hawk'-beard, 1161.

Hawthorn, 738 .

Hazel-nut, 1403.

Heart's-ease, 347 .

Heath, 603 .

Hedye Bed-straw, 239.

Hedge-mustard, 988.

Hellebore, 856.

Helleborine, 1287.

Hemlock, Commou, 445 Water, 443.

Hemp-agrimony, 1191.

Hemp-nettle, 888.

Henbane, 356.

Herb Bennet, 797. Christopher, 801. Paris, 621.

Robert, 1026.

High taper, 349 .

Hogweed, 476.

Holly, 261.

Holy-grass, 135.

Honewort, 441.

Honeysuckle, 365.

Hop, 1472.

Horehound, Black, 899.

Hornbeam, 1401 .

Horned Pondweed, 1377.

$$
\text { Poppy. } 803 \text {. }
$$

Horse-radish, 971.

Hound's-tongue, 295.

House-leek, 732 .

Ivy, 377.

Jack-by-the-Hedge, 994.

Jacob's Ladder, 325.

Jointed Clıarlock, 1017.

Jointed Glasswort, 1.

Juniper, 1482.

Kidney Vetch, 1054.

Kipper, 434.
Knap-weed, 1253.

Knawel, 657.

Knot-grass, 378.

Ladies'-finger, 1054.

- Mantle, 257.

_ Slipper, 1296.

- smock, 979.

Lames, 1289.

Lamb's Lettuce, 57.

Lancashire Boy-asphodel, 523.

Lark:jpur, 826.

Leopard's-bane, 1236.

Lettuce, 1132.

Lily of the Valley, 525 .

Lime-tree, 818.

Liug, 602.

Lolıdon-rocket, 989.

Louse-wort, 924.

Lucerne, 1107.

Lung-wort, 297.

Madder, Wild, 243.

Mallow, 1036.

Manna-grass, 141.

Maple, 609 .

Mare's-tail, 5.

Marjoram, 903.

Marsh Fel-wort, 408.

- Flea-wort, 1234.

_ - Mallow, 1035.

- marigold, 858.

Master-wort, Great, 456.

Mat-grass, 94.

May-weed, 1241.

Meadow Clary, 49

- Fox-tail-yrass, 103. grass, 146.

- rue, 835 .

saffron, 578 .

sweet, 748 .

Medick, 1107.

Medlar, 739.

Melancholy Thistle, 1184.

Melic-grass, 136.

Melilot, 1085.

Mercury, 1479.

Meu, 461.

Mezereon, 607.

Mignonette, wild, 7:0.

Milfoil, 1251.

Milk Thistle, 1178.

Milk-wort, 1046.

Mint, Bergamot, 873.

- Horse, 869.

- Pepper, 872.

-, Spear, 871.

Misseltoe, 1469.

Mithridate, 960 .

Money-wort, 317.

Monks'-hood, 827.

Moon daisy, 1238.

Moor-grass, 139.

Moss Campion, 674.

Mother-wort, 901. 
Mountain-ash, $7 \$ 5$.

Mountain-sorrel, 563.

Mouse-ear, 714.

Mouse-tail, 498.

Mud-wort, 941.

MIng-wort, 1199.

Mug-weed, 226.

Mullein, 349.

Musk Thistle, 1175.

Mustard, brown, 1014.

Narcissus, 501. white, 1013.

Navel-wort, 694.

Nep, 867.

Nettle-great, 1383. Roman, 1381. small, 1382.

Night-shade, 358.

Nipple-wort, 1168.

Oak, 1395.

Oat-wild, 187.

Orache, 1485.

Orchis.

\begin{tabular}{l} 
Bee, 1277. \\
Bog, 1293. \\
Fly, 1276. \\
\hline late-spider, 1278. \\
Orpine, 696.
\end{tabular}

Osier, common, 1463.

- golden, 1420 .

Ox-lip, 306.

Ox-tongue, 1126.

Poony, 825.

Paigle, 307.

Pansy, 347.

Parsley Piert, 259.

Parsnep, 475.

Pasque-flower, 830.

Paul's Betony, 893.

Pea, 1055.

Pearl-wort, 276.

Pear Tree, 741.

Penny-cress, 963.

Penny-royal, 881.

Periwinkle, 381 .

Persian-willow, 586.

Perscicaria, 611.

Petty-whin, 1052.

Pheasant's eye, 839 .

Pig-nut, 434.

Pile-wort, 843.

Piony, 825.

Pimpernel, 318.

Pink Clove, 662.

- Deptford, 660 .

Pipe-wort, 1387.

Plantain, 245.

Plantain shore-weed, 1378.

Plowman's spikenard, 1211

Plum, 735.

Poplar, black, 1477.
Poplar, white, 1474.

Poppy, 806.

Prickly Samphire, 418.

Primrose, 305.

Privet, 15. peerless, 502 .

Quaking-grass, 156.

Queenof the Meadows, 750 .

Quicken Tree, 745.

Radish, 1017.

Ragged Robin, 710.

Rag-wort, 1222.

Rampion, 336.

Ramsons, 504.

Rape, 1007.

Raspberry, 776.

Red-rattle, 924.

Reed-mace, 1312

Rest Harrow, 1053.

Roan Tree, 745.

Roast. Beef plant, 65 .

Rock-rose, 818.

Rose, 751.

Rose-root, 1478.

Rupture-wort, 383.

Rush, 530.

Rye-grass, 199.

Saintfoin, 1080.

St. Barnaby's thistle, 1259.

St. John's wort, 1113.

St. Peter's wort, 1115.

Saffron-crocus, 59.

Sallow-thorn, 1470.

Salt-wort, 399.

Samphire, 452.

Sanicle-wood, 417.

Sauce-alone, 994 .

Saw-wort, 1173.

Saxifrage, 637.

Scotch Fir, 1403.

Scottish asphodel, 575.

Scurvy-grass, 967.

Sea Beet, 398.

-Buckthorn, 1470.

- Hard-grass, 202.

Heath, 560 .

Holly, 415.

Kale, 977.

- Lavender, 488.

Lyme-grass, 203.

Milk-wort, 379.

-_Purslane, 1485.

Reed, 198.

Rocket, 976.

Star, 1228.

_-Sulphur-wort, 774 .

Sedge, 1318.

Self-heal, 912.

Service Tree, 743.

Sheep's-bit, 337.

Shepherd's needle, 427 . Purse, 966. Staff, 219. 
Shore-weed, 1378.

Skull-cap, 910.

Sloe, 737.

Slough-heal, 912.

Snake-weed, 616.

Snap-dragon, 932.

Snow-drop, 499.

Snow-flake, 500.

Soap-wort, 659.

Soft-yrass, 132.

Solomon's seal, 526 .

Sorrel, 572.

Southern-wood, 1195.

Sow-thistle, 1128.

Speedwell, 20.

Spignel, 461.

Spikenard, 1211.

Spindle Tree, 370.

Spurge, 1298.

Spurge, olive, 607.

Spurge, laurel, 608.

Spurrey, 722.

Squinancy -wort, 225.

Squirrel-tail-grass, 208.

Star of the Earth, 249. of Bethlehem, 513.

Star-thistle, 1257.

Starch Hyacinth, 52l.

Stitch-wort, 675 .

Stock, 997.

Stork's-bill, 1019.

Strap-wort, 485.

Strawberry Hautboy, 783 .

$$
\text { Tree, } 627 .
$$

Wood, 782.

Succory, 1170.

Sundew, 495.

Sweet Alyssum, 955.

Cicely, 430.

Flag, 529.

Gale, 1471.

Sweet-scented Vernal grass, 52.

Swine's Succory, 1169.

Sycamore, 609.

Tamarisk, 484.

Tansy, 1194.

Tare, 1076.

Thorn-apple, 355.

Thorough-wax, 469.

Thrift, 489 .

Thyme, 904.

Timothy-grass, 97.

Toad-flax, 930.

Tooth-wort, 923.

Touch-me-not, 340 .

Tower mustard, 1006.

Traveller's Joy, 834.

Tulip, 512.

Turnip, 1008.

Tutsan, 1114 .

Tway-blade, 1284
Valerian, 53.

Venus's Comb, 427 .

Vervain, 868.

Veteh, 1066.

Violet, 341.

Viper's Bugloss, 304.

Wake Robin, 1393.

Wall-Hower, 993.

Wall-lettuce, 1135.

Wall-pellitory, 255.

Water aloe, 829. -Betony, 935.

Blinks, 214.

Water Cress, 984.

Water Drop-wort, 447. Featherfoil, 313. Hair-grass, 131. Lily, 812.

Water Milfoil, 1390.

Water-parsnep, 435.

Water-plantain, 579 .

Water-soldier, 829.

Water Star-wort, 13.

Water Violet, 313.

Water-wort, 623.

Wayfaring Tree, 479.

Weld, 730 .

Whin, 1051.

Whip-tongue, 239.

White Briony, 1386.

White Mullein, 350.

White Ox-eye, 1238.

White rot, 472 .

Whitlow grass, 950 .

Wild Basil, 902.

- - Navew, 1009.

_ Rosemary, 626. Succory, 1170.

Willow Bedford, 1423. - rose, 1425.

- crack, 1422 . varnished, 1421.

White Welch, 1421.

Winter-green, 630.

Wolf's-bane, 827.

Woodbine, 366.

Woodruff, 224 .

Wood Sage, 864.

Sorral, 707.

Wormwood, 1198.

Yarrow, 1251.

Yellow archangel, 892.

Yellow Bird's nest, 625 .

—_ Bugle, 863.

_._. Loosestrife, 314.

— Ox-eye, 1239.

- Pimpernel, 316.

Water Lily, 813.

Yellow-weed, 703.

Yew, common, 1484. 




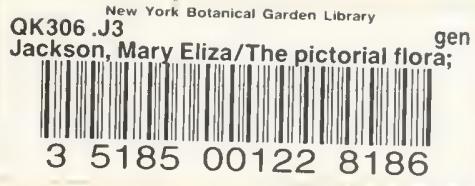




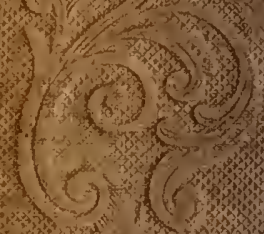

(c) ce)

(cis

$\left.\left(\frac{1}{2}\right) c^{2}\right)$

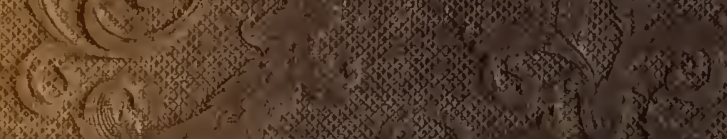

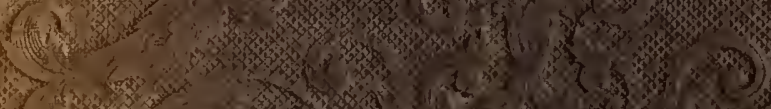

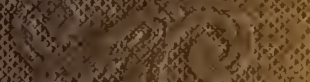

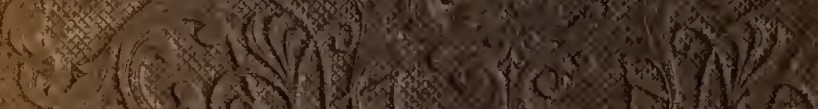

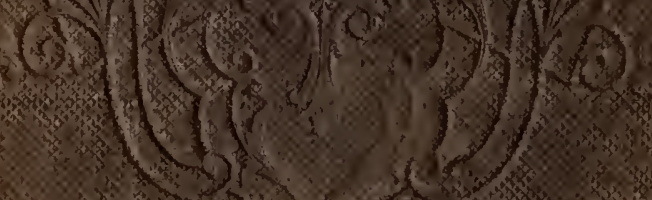

